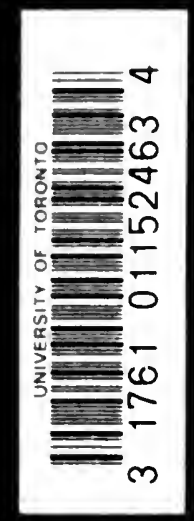




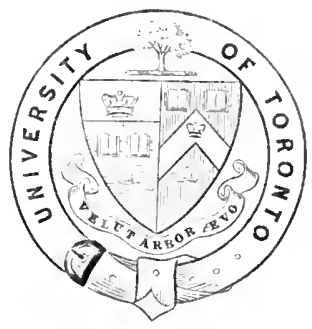

Drescnted to

\section{The Tibrarp}

of the

\section{Aniversity of Toronto}

P.H. Walks Esq. 


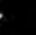



RHWWltes Univ. Coll. Hor. 91-2. 



\section{Digitized by the Internet Archive in 2007 with funding from Microsoft Corporation}




\section{OPINIONS OF THE FIRST VOLUME \\ or}

\section{PROFESSOR BALDWIN'S PSYCHOLOGY}

(Handbook of Psychology : Senses and Intellect).

Revue Phrlosopmeue:-An excellent treatise on psychology, superior, and much superior, to perhaps any other that we know.

NATURE:-Well arranged, carefully thought out, clearly and tersely written, it will be welcomed in this country as it has been welcomed in America. That it views psychology from a standpoint somewhat different from that which Mr. Sully takes up in his outlines, will render it none the less acceptable to English students.

Edinburat Scotsman:-The work is one of the most noteworthy that have appeared in recent times to vindicate the claims and establish the position of Psychology as an independent science.

NATION (New York):-Taken as a whole, it is about the best we know.

OxFord MAgAzINE:-Already in its second edition, and thoroughly deserves that honor. It excels just where Prof. James' fails. Senses and Intellect is the best manual we have seen, and we look forward to the companion volume.

Professor WUNDT:-I am impressed with its clear arrangement and logical treatment. . . The book will serve a high purpose for students both as introduction to the subject and as preparation for original work.

MIND:-The opportunity may be seized ( $2 \mathrm{~d}$ ed.) to recommend the book with some more emphasis as a very serviceable manual for students.

Boston Post:-It is altogether a scholarly work, and done in a thoroughly scientific spirit which is as welcome as it is rare.

CrITIC (New York):-Thorough equipment, full knowledge up to date of his subject, in general welldigested presentation, cautious valuation, and honest intent in interpretation, entitle the author's work to respectful praise.

NEW YORK: HENRY HOLT \& ĊO., PUBLISHERS. LONDON: MACMILLAN \& CO. 


\title{
HANDBOOK OF PSYCHOLOGY
}

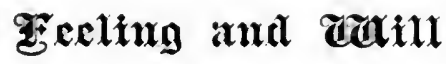

BY

JAMES MARK BALDWIN, M.A., РH.D.,

PROFESSOR IN THE UNIVERSITY OF TORONTO; AUTHOR OF

"HANDBOOK OF PSYCHOLOGY : SENSES AND INTELLECT"
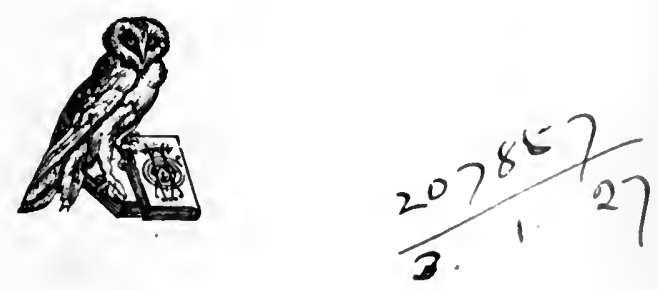

NEW YORK

HENRY HOLT AND COMPANY 
Copyright, 1891,

BY

HENRY HOLT \& CO.

[All rights reserved.]

Robert Drummond,

Electrotyper aNd Printer, New Yorr. 


\section{PREFACE.}

THE present volume does not need a preface. It completes the survey of the mind begun in my "Handbook of Psychology : Senses and Intellect." In method and scope my plan has remained the same. The treatment of this volume, however, is somewhat fuller: since I have wished to remove, in some degree, the reproach so often and so justly cast upon the general works in Psychology that they give Feeling and Will summary and inadequate discussion. Indeed, I have found the field so inviting and the data so rich that the self-control made necessary by the limits of the space available has been more difficult than with the earlier volume.

This volume, it may be said, however, puts to a better test the claim upon which the Handbook is written, i.e., the possibility of a psychology which is not a metaphysics, nor even a philosophy. For the phenomena of the emotional and volitional life have not been worked over for purposes of philosophical system, as intellectual phenomena have: and for this reason, the psychologist has in this field greater freedom of treatment and a larger scientific opportunity. Hence-while not laying a claim to originality, which only the opinion of competent readers could make of any force-I feel that, apart from the general arrangement and division, certain chapters

${ }^{1}$ New York, Henry Holt \& Co., 1889; second edition, revised, 1890; London, Macmillan \& Co., 1890. 
of this volume are more independent. In other words, the book not only aims to be useful for purposes of university instruction, but it may also be found, on some points, to make cơntributions to psychological discussion. The topics to which I refer especially are: "Interest, Reality, and Belief" (Chap. VII), "Pleasure and Pain" (Chaps. V and XI), "Conceptual Feeling" (Chap. IX), "Suggestion as Motor Stimulus" (Chap. XIII, §1), and the theory of "Volition" (Chaps. XV and XVI). A point of distinctive treatment under the head of Will is the emphasis laid upon the analysis of the "Reactive Consciousness" considered as basis of Volition. Teachers who use the work for a systematic course of instruction may find it advantageous to take up the Introduction (Chaps. I-III) to the second volume immediately after the Introduction (Chaps. I-III) to the first volume, before proceeding to study the Intellect.

I am indebted for several points of fact, made use of in the chapter on "The Nervous System," to my colleague in Physiology, Dr. A. B. Macallum. Other obligations have acknowledgment in their proper places in the text.

I trust that the completed work may justify the cordial reception which the first volume has already had, and be of use in bringing about a better understanding among psychologists.

J. Mari BaLdwin.

Toronto, September, 1891. 


\title{
CON'TEN'TS.
}

\section{PART III.}

FEELING.

\section{GENERAL INTRODUCTION TO FEELING.}

\author{
CHAPTER I.
}

The Nervous System.

\$1. Structure of the Nervous System. - General conception of a nervous system. - Nerve-elements. -Combination of elements into a system.-The receiving apparatus. - The reacting apparatus. - The registering apparatus,

82. Functions of the Nervous System.-Fundamental properties of nervous tissue. - Neurility. - Sentience: integration, retention, selection.-Law of nerrous dynamogenesis,

§ 3. Kinds of Nerwous Reaction.-Automatic.-Reflex.Voluntary.-Negative: inhibition,

§4. Principles of Nervous Action.-Specialization.-Indifference.-Substitution. - Specific connection.Summation

\$ 5. Final Statement of Nervous Function.-Habit.-Accommodation.-Inheritance, . . . . .

\section{CHAPTER II.}

\section{The Nervous System and Consciousness.}

81. Nervous Conditions of Consciousness, . . . 51-55

\$2. Sentience and Sensibility.-Sentience and the subconscions, 
§3. Kinds of Consciousness as dependent on Nervous PAGES Function.-Passive consciousness.-Reactive consciousness.-Voluntary consciousness, . . .

§ 4. Analogies of Function. - Nervous retention and consciousness.-Specific nervous connection and consciousness.-Nervous summation and consciousness. -Inhibition and consciousness

\$ 5. The Nervous System and the Unity of Consciousness,

\$. Organic Theory of the Unity of Consciousness,. .

\$ 7. Heredity and Consciousness, . . . . . 81-83

CHAPTER III.

Nature and Divisions of Sensibility.

§1. Nature of Sensibility.-Definition.-Most general mark of sensibility,

§ 2. Divisions of Sensibility, . . . . . . . . . . 88

\section{SENSUOUS FEELING.}

CHAPTER IV.

\section{Sensation and Common Feeling.}

§1. Sensations as Forms of Sensibility.-Relativity of sense-qualities: contrast.-Relation of sensation to knowledge.-Extensity of feeling, . . . .

§2. Common Sensuous Feeling.-Divisions of common feeling.-Organic feelings.-Cutaneous feelings.Muscular feelings. - Kinæsthetic feelings.-Feelings of innervation.-Sequence of innervation-feelings. -Nervous feelings. - Physiological proof of distinct common feelings,

\section{CHAPTER V.}

\section{Sensuous Pleasure and Pain.}

§1. Physical Conditions of Pleasure and Pain.-Empirical characters of pain.-Analgesia.-Pain as feeling and as tone.-Relativity of sensuous pleasure and pain, . . . . . . . . . 113-122

§2. Conception of Pleasure and Pain.-General conclusion.-Interpretation of relativity, . . . . 122-127

§ 3. Theories of Sensuous Tone, . . . . . . 127-133 §4. Pleasure and Pain as Worth, . . . . . 133-134 


\section{IDEAL FEELING.}

CHAPTER VI.

Nature and Divisions of Ideal Feeling.

PAGEB

\$1. Nature.-Ideal vs. sensuous feelings, . . . . 135-136

§ 2. Division.-Ideal feelings as special and common.-

Ideal pleasure and pain, . . . . . 136-137

\section{CHAPTER VII.}

Common Ideal Feeling: Interest, Reality, and Belief.

§1. General Character of Common Ideal Feeling: Interest. - Physiological basis of interest and indifference.Intellectual conditions of interest.-Interest of discrimination.-Emotional and active intercst.-Interest of custom or habit.-Association and interest. -Definition of interest. - Intercst as ideal emotion.

-Interests vs. affects, . . . . . . . . . 138-148

§. Reality-feeling.-Distinction between belief and reality-feeling.-Rise of reality-feeling.--Rise of unreality-feeling. -Degrees of reality- and unrealityfeeling. - Time and space reference of reality-feeling, 148-155

83. Belief.-Doubt.-Development of doubt.-Resolution of doubt. - Nature of belief.-Reaction of belief on reality.-Kinds of belief,

\$ 4. Belief in External Reality.-Its coefficient.-Primacy of muscular sensations.-Criteria of external reality, 160-163

§ 5. Belief in Memory.-Memory-coefficient.-Completed criterion of external reality, . . . . . 163-166

§ 6. Belief in Concepts and Thought.-Thought-coefficient, 167

\& 7. Emotional Belief, . . . . . . . . 168

\$ 8. General Conclusion.-Composito realities.-Delf the ultimate reality.-Existence. - Relation of belief to will.-Definition of belief.-Interest and belief, . 168-173

\section{SPECIAL IDEAL FEELINGS.}

QUALITY, OR KINDS.

CHAPTER VIII.

Division : Presentative Emotions.

\$1. Division.-General nature.-Kinds, . . . . 174-175

$\$ 2$. Emotions of Activity-of adjustment-of function . 175-177 
\$3. Emotions of Content, . . . . . . . . 177-178

§ 4. Self-emotions.-Egoistic, . . . . . . . 179-180

\$5. Objective Emotions, . . . . . . . . 180-181

§6. Expressive Emotions.-Emotions of attraction-of repulsion, . . $\cdot$. . . . . . 182-186

§7. Sympathetic Emotion.-Definition of. sympathy.Kinds of sympathetic suggestion.-Development of sympathy.-Altruistic element.-Varieties of sympathetic emotion.-Social feeling, . . . . 186-193

§ 8. Representative Emotion, . . . . . . . . 193-194

\section{CHAPTER IX.}

\section{EMotions of RELATION.}

§1. Logical Emotions, . . . . . . . . 195 198:

§ 2. Conceptual Emotions, . . . . . . 198

§3. Construction of Ideals.-Nature of ideals.-Feeling of fitness, . . . . . . . . . 198-202

§ 4. Conceptual Feeling as Intuition, . . . . . 202-204

§5. Range and Kinds of Conceptual Feeling, . . . 204

\$ 6. Feelings for System. - Scientific system.-Philosophical system, . . . . . . . . . . 204-205

§7. Ethical Feeling.-Its coefficient.-Moral quality.Moral quality as harmony-as universality.Moral sympathy.-Moral authority.-Grounds of moral authority.-Conclusion on moral coefficient. - Moral ideal: ethical end.-Notion of end.-Subjective vs. objective end.-Moral vs. natural end. -Theories of end.-Happiness theories. - Utility theories. - Criticism. - Kantian theory. - Intuitional theory.-Relative theories.-Conclusion on end.-Rules of conduct.-Moral judgments.-Conscience.-Ethical feeling and happiness.-Emotions akin to the moral, . . . . . . . 205-233:

§ 8. Fisthetic Feelings. - Lower. - Higher. - Beauty of truth - of character.-Meaning in the arts.Assthetic judgment universal.-Emotions allied to the æsthetic,

§9. General Table of Feelings, 


\section{CHAPTER X.}

Quantity and Duration of Emotion.

§1. Quantity, or Intensity.-Mental exeitement.-Relativ-

PAGES. ity of feeling. - Principle of contrast-of attention - of accommodation-of fatigue.-Emotional expression.-Theories of expression. - Physical basis of emotion.-Diffusion of emotion.-Brain-seat.Passion.-Theories of emotion.-Reproduction of emotion.-Transfer by association.-Conflict of

\$2. Duration of Emotion.-Cessation and relief. Rhythm, . . . . . . . . 263-265

CHAPTER XI.

Ideal Pleasure and Pain.

\$1. Primary Conditions of Ideal Tone, . . . . 266-268

\$2. Secondary Conditions, . . . . . . 268-274

\$ 3. Conclusion, . . . . . . . . 274-275

§4. Universality of Tone.-Complexity of tone-states.-

Relativity of ideal pleasure and pain, . . . 275-279

\section{PART IV.}

\section{WILL.}

\section{MOTOR ASPECTS OF SENSUOUS FEELING.}

\section{CHAPTER XII.}

The Motor Conscrousness.

\$1. Idea of the Motor Consciousness. - Law of mental dynamogenesis. - Varieties of motor consciousness, . 280-282

\$2. Motor Value of the Subconscious, . . . . 283-284.

\$3. Motor Value of Reactive Consciousness.-Elements of reactive consciousness, . . . . . . 285-286.

\$ 4. Feeling of Expenditure in Attention.-Sensorial and intellectual attention, . $. \quad . \quad . \quad . \quad$ 286-289 
\$. Theories of the Feeling of Activity in Reflex AttenPAGES tion.-Effect theory of reflex attention.-Spiritual theory,

\$. Conclusion on Reactive Consciousness, . . . . 293-294 CHAPTER XIII.

Stimuli to Involuntary Movement.

§1. Notion of Stimulus.-Kinds of motor stimuli._Extraorganic stimuli.-Reflex stimuli.-Suggestion as motor stimulus. -Organic stimuli.-Expressive reactions.-Pleasure and pain as stimuli.-Nature of pleasure and pain reactions.-Motor spontaneity, . 295-304

§ 2. Impulse and Instinct.-Impulse.-Definition.-Instinct.-Complexity of instinct.-Definition of instinct. - Variability of instinct, . . . . 304-313

§3. Affective Nature of all stimuli to Movement.-Affects.-Division of affects, . . . . . 313-315

\section{MOTOR ASPECTS OF IDEAL FEELING.}

\section{CHAPTER XIV.}

\section{Stimuli to Voluntary Movement.}

§ 1. The Voluntary Motor Consciousness: General stimuli.-Interest.-Affects as voluntary stimuli, 316-320

§2. Sperial Stimulus: Desire.-Impulse in desire.Kinds of intellectual impulse : appetence.-Desire and its objects.-Tone of desire.-Coefficient of desire.-Physical basis of desire.-Aversion, . . 320-331

§3. Voluntary Movement and Habit, . . . . 331-332

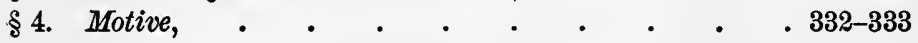
CHAPTER XV.

\section{Voluntary Movement.}

\$1. Feelings of Effort and Consent.-Fiat, psychological and physiological.-Neget, psychological and physiological.-Consent.-Summary on muscular effort. -Muscular effort and the attention, . . . 334-344

§2. Physiology of Voluntary Movement.-Psycho-physical conception of will.-Theory of innervation, . 344-350 


\section{CHAPTER XVI.}

VOLITION.

§1. Purpose: Voluntary Attention as Choice.-Law of

PAOES motive. - Nature of motives. - Volitional appercoption.-Controlling motive.-Deliberation.-Choice. - Potential and final choice.-Feeling of alternatives.-Moral choice.-Choice and habit.-Intellectual effort, . . . . . . . . 351-363

\$2. Character. - Development of character, through choice, . . . . . . . . . 363-367

\$ 3. Initiation of Motives by Attention, . . . . . 367-369

\$4. Freedom of the Will.-Indeterminism.-External determinism.-Immanent determinism.-Freedom as self-expression.-Feeling of freedom-of responsibility,

85. Effects of Volition.-Expressive effects. - Effects proper.-Physical control.-Mental and moral control, . 376-381

§ 6. Rational Aspects of Volition.-Intuition of power.Intuition of obligation, . . . . . . . 381-382

APPEndix A.-Educational Psychology: Bibliography, . 383-384 APPENDIX B.-Dream-excitation, . . . . . 384-385 APPENDIX C.-Number-forms, . : . . . . . 385-387 INDEX, $\quad . \quad \cdot \quad \cdot \quad \cdot \quad \cdot \quad \cdot \quad \cdot \quad \cdot \quad \cdot 389$ 



\title{
PART III.
}

\author{
FEELING.
}

GENERAL INTRODUCTION TO FEELING.

\section{CHAPTER I.}

THE NERVOUS SYSTEM.

THE intimate connection between mind and body which has been already assumed in the treatment of the intellectual function, and which was appealed to in support of several important interpretations there, comes before us at the outset of any thorough examination of our present subject. It is only necessary to recall the truths of the sensational life to be aware also that it is in the realm of feeling that this connection has its fundamental meaning. Sensations we found to be the first content or filling of the apperceptive process, and sensations are at least-whatever else they may he held to be -the inner accompaniment of a physiological process. Sensations are first of all affective. The claim that physiology and pathology have upon us, therefore, in this connection, must have at the outset somewhat extended recognition.

The recognition thus given in what follows, however, is purely psychological recognition. It would be entirely gratuitous, as well as misleading, to burden an exposition of mental 
processes with a mass of detailed physiological data. This extreme of politeness and hospitality to a sister-science would be more just to ourselves and more complimentary to her if it took the form of an earnest study of her results as she reports them, rather than the attempt to condense them into a kind of psychological introduction. This has been done too much in the "physiological psychologies." The following pages, therefore, aim to present only the great outline principles of nerve-physiology which press into the psychological arena with a claim, at least, for recognition, as having more than physiological value.'

The reader should also recall here the general postulate found necessary to a consistent conception of true psychological method, namely, the postulate that all mental processes are correlated with physical changes ; that there is an uniform psycho-physical connection. ${ }^{2}$ This assumed, we may construe physiological data as causes or effects relative to affective data in our attempt to unify and explain the mental life.

\section{§1. Structure of the Nervous System.}

General Conception of a Nervous System. The problem which a nervous system wherever found may be said more or less perfectly to solve may be stated thus: Given a set of mechanical conditions, to devise an apparatus which shall receive, register, and react upon these conditions severally, in such a way that, while adapting itself progressively to greater complexity of condition, it at the same time realizes in itself a higher integration of the same conditions. The nervous system probably took its rise as a new integration of the modes of mechanical movement. The question as to how such an integration is possiblewhether a new agency altogether, life, organism, or more, was necessary for the result-is not before us here. The essential thing is twofold : first, that the nervous system is itself part of nature, is mechanical in its function ; and second, that it is an integrated thing, which in com-

\footnotetext{
${ }^{1}$ For general works on nerve-physiology, see references at the end of this chapter.

Senses and Intellect, $2 \mathrm{~d}$ ed., p. 28.
} 
plexity, and consequently in function, finds no complete analogy in anything else in nature.

The mechanical conception of the nervous system is becoming an axiom among those competent to form a couception from the facts at hand. The old conception of the system as a mysterious generator of force, which shed its energies into nature through muscular move- ment, finds few modern adherents. The activities of the nervous system as such, apart from those higher functions in which consciousness has a share, show it to be a machine. Electrical experiments realize most perfectly the purity of nerve-action considered as mechanical.

It may be unnecessary to note that the mechanical conception of the nervous system has no question-begging bearing upon the subsequent theory of voluntary movement. Whatever the will be, the nervous system is what it is. If we find reason to believe that the will is a new force, then voluntary action is nervous action plus something else : if the will turn out to be a conscious phase of nervous change, then only does the mechanical interpretation swallow it up. The questions may be separated clearly enough at least for provisional treatment, and in the discussion of the will the points of real contact and issue will become plain.

The other essential feature of a nervous system, its complexity as resulting from progressive adaptation to a mechanical environment, will detain us longer. The history of such a development in complexity is not within our province : it belongs to general biology and embryology. But the fact of such complexity is of the utmost moment to pure psychology. When we remember that the mental life is a complexity of unheard-of

- intricacy, that introspective analysis is often powerless to separate it into more than the roughest groups of elements, we are prepared to discover a similar state of things in the case of its physical basis. How can I analyze mental processes? Analyze the nervous system, 
is at least a partial answer : an answer which is true to some extent whatever theory we ultimately hold of the nature of mind. By way of illustration, the localization of cerebral functions may be cited as an instance of nervous analysis which carries with it mental analysis. The determination of the different centres in the cortex corresponding to different classes of memories (of words as heard, as spoken, as seen, etc.) has given us an analysis of the so-called "faculty of speech" of the old phrenology.

Taking the two general characteristics thus arrived at, complexity and mechanism, we may inquire more particularly into: I. The elements of the nervous system and their properties, both as elements and as a system; and, II. The activities of the nervous system, or the modes of nervous action.

I. Nerve-elements. As far as our knowledge goes, we are able to make a twofold distinction among the elements called nervous, nerve-fibres and nerve-cells. As to what these are, the general meaning ordinarily attached to the words expresses about the amount of knowledge physiologists possess. That is, a nerve-fibre is a thread-like connection between different muscular and cellular masses, in parts, at least, longitudinally homogeneous and continuous. A greater or smaller number of these white thread-like fibres may unite together to constitute a "nerve," which connects an organ (muscle, gland, etc.) with a greater or smaller mass of cells. The cells, on the other hand, are microscopic elements shaped like a flask or long-necked squash. One of the necks-for there may be more than one-seems to be prolonged into the fibre, and is called the axis-cylinder process of the cell. Both cells and nerves have nuclei, small dark points which are surrounded by protoplasm. 'The nerves are also cut up at intervals by nodes resem- 
bling the divisions in a length of corn-stalk. See Figs. 1 and 2.

Some cells, however, are found without such connections, as far as microscopic analysis is able to go. The cells are largely gathered in masses or "centres,"

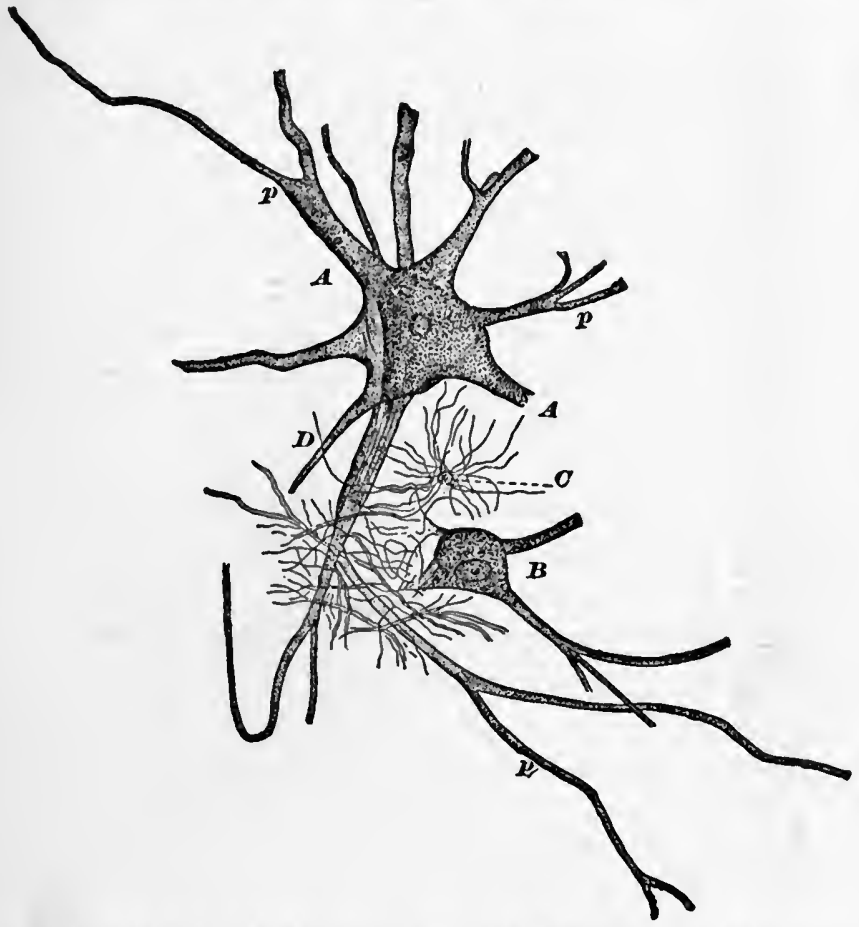

Fro. 1.-From a piece of spinal cord. $A$ and $B$,ganglion-cells; at $D$, axis-cylinder; $p$, protoplasmic process; $c$, neuroglia-celis. (Atter Ranvier, from Edinger,

toward which fibres from different regions or organs converge and apparently lose themselves. What is usually called the brain is a series of such centres, varying in size and complexity from the cerebral cortex downward into the spinal cord. In the centres the cells are separated by a substance called neuroglia (see Fig. 1) which may be simply a form of insulating or connective 


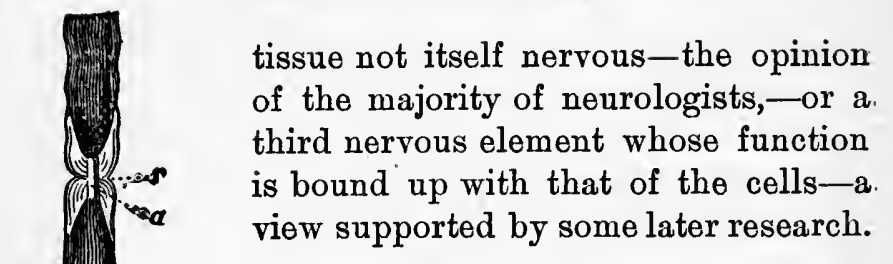

Farther and more minute questions, such as the chemical composition, methods of insulation, processes of growth and decay, of the nervous elements, need no notice here. It is safe to say that as yet. they have no psychological significance, not perhaps as safe to say that they never will.

Late researches are showing that the connection between nerves and cells is a more complex matter than the above account indicates. In very few cases is there reliable evidence of direct continuity between the axis-cylinder process of a cell and the axis-cylinder of a nerve. On the contrary, the researches of $\mathrm{His}^{1}$ Golgi, Kölliker, ${ }^{2}$ and others, tend to show that both the processes of the cells and the subdivisions of the nerves divide again and again, to be lost in the interlacing fibrillar network which the neuroglia supports. Kölliker goes so far as to say, speaking of one of the layers of the small brain: "No anastomosis can be discovered between the endings of cells or fibres with themselves or with one another." His reaches the same conclusion regarding the brain and spinal cord generally, though Köllikerfinds motor cells in the cord-cells whose

FIG. 2. - Nerve-flbres (after Schwalbe). $a$, Axis-cylinder; $s$, sheath of Schwann: $n$, nucleus; $p$, granular substance at the poles of the nucleus; $r$, Rauvier's nodes, where the medullary sheath is interrupted and the axis-cylinder appears. processes are continuous with the motor nerves.

Combination of Nerve-elements in a system. The elements spoken of somewhat artificially as cells and fibres.

${ }^{1}$ Archiv für Anat. u. Phys., Anat. Abth., Supplement Band, 1890.

${ }^{2}$ Zeitschrift für wissenschaftliche Zoologie, Mai und Dec., 1890. Resumed by Donaldson, Amer. Journ. Psych., III. pp. 544-48. 
have no functional existence apart from each other and from the living organism as a whole. Viewed as a whole, as receiving, registering, and reacting upon stimuli, they constitute the nervous system. As a system, the nervous apparatus is essential to the life of a higher organism and partakes with it of a great differentiation of parts. What we call organs or members of the body have a unity of their own structurally : but their functional activity is one with the general life-process of the whole. So the organs or members of the nervous system have a corresponding structural differentiation. Whether the three general functions of the system spoken of above, receiving, registering, and reacting upon stimuli, are in any way adequate as a functional conception or not, they will at any rate serve to guide us in describing the three great parts or divisions of the nerve-apparatus. We will accordingly say a word about these three divisions in order.

The Receiving or Sensor Apparatus. By this is meant that part of the nervous system which is normally concerned with stimuli from without. We say normally concerned, since there is reason to believe that all nervetissue has the receiving property. But we find a great system of fibrous pathways arranged for the evident purpose of propagating disturbances from the periphery of the body and from various organs, to the higher centres. Further, these fibrous pathways may have special receiving organs exposed to the peculiar stimulus which we call psychologically the stimulus to a particular sensation: such special organs being peculiar to the special senses, as the eye for sight, ear for hearing, etc. Accordingly, the receiving apparatus includes two distinct elements, the sensor course and the end-organ. The latter (say the eye) receives some form of excitation (light), and the former (optic nerve) propagates it to the brain. 
The existence of sensor courses which have no endorgans is sufficient to show that the latter is not a necessary part of the system, except when the system is highly differentiated. A sensor nerve may be stimulated mechanically by a blow, by a touch upon an exposed point, etc., even in the case of the nerves of special sense; they then report the sensations ordinarily secured through their end-organs. ${ }^{1}$

The nerves of special sense show no structural peculiarities except the possession of the end-organ. By nerves of special sense are meant those which report sensations recognized and classed as having distinct psychological quality. That is, we find special end-organs for each of the seven classes of sensations discussed under the head of the presentative function, the muscles being considered end-organs of the muscular sense.

Besides these, there is a mass of nerve-courses which report less distinctly differentiated and localized stimuli, the purest and most general psychological condition that they induce being pleasure and pain. These are called general as opposed to the special courses, and constitute the physiological basis of the general sensibility.

As to distribution, the sensor apparatus is coincident in extent with the body itself. The organs of general sensibility are distributed throughout in the form of very fine fibrils; these fibrils being gathered into bundles and these again into larger bundles or nerves as they approach the central course, the spinal cord. With these are the nerves of touch and muscular movement, also of general distribution, the whole being consolidated into two columns which form part of the white matter of the spinal cord. The posterior or dorsal portion of the cord (the portion farther back in man and up in animals) is called the sensor portion (postero-median

\footnotetext{
1 For example, sparks of light which result from a blow on the optic nerve or from mechanical irritation of a blind eye.
} 
columns). After gathering up representative fibres from all the successive nerves of sense which run into the spinal cord, these tracts terminate in the upper enlargement of the cord (medulla); but farther pathways lead up to the highest centre, the cortex of the brainand this is the essential point. For the location of these tracts in the cord, see Fig. 3.

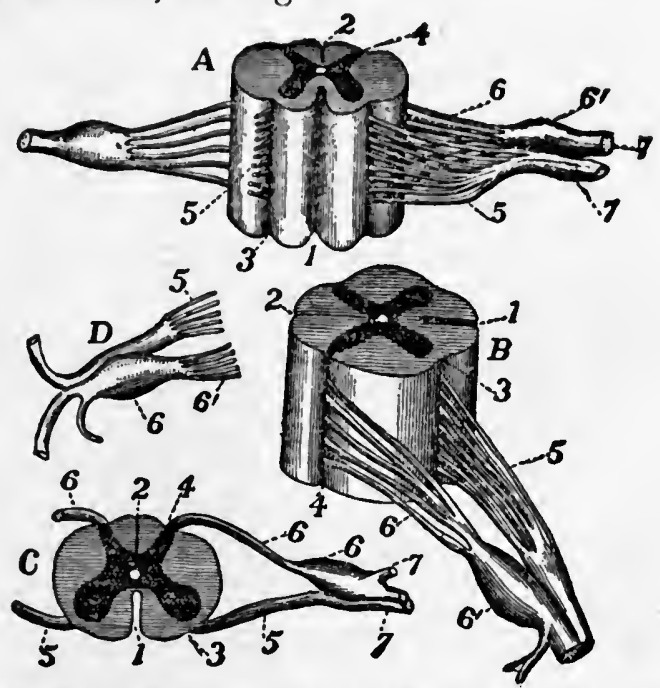

Fig. 3.-Tlıe sninal cord and nerve-roots. $A$, a small portion of the cord seen from the ventral side; $B$, the same seen laterally: $C$, a cross-section of the cord; $D$, the two roots of a spinal nerve; 1 , anterior (ventral) fissure; 2 , posterior (dorsal) flssure and columns; 3, surface groove along the line of attachment of the anterior nerve-roots; 4, line of origin of the posterior roots; 5, anterior rootflaments of a spinal nerve; 6 , posterior root-filaments; 6 , ganglion of the posterior root; $7,7^{\prime}$, the first two divislons of the nerve-trunk after its formation by the union of the two roots.

Another tract (the cerebellar) is also supposed to carry incoming impulses upward: it arises from cells distributed along the cord and passes continuously to the cerebellum (little brain). As the cerebellum is also in direct connection with the hemispheres, another upward path is thus established. Foster further supposez that incoming impulses may travel by the gray matter of the cord (see below), or by portions of the gray matter with the longitudinal fibres which connect different segments of the cord together. ${ }^{2}$

${ }^{1}$ Text-book of Physiology, 5th ed., pt. III. p. 1104. Cf. his whole discussion, ibid., sect. 9. 
The arrangement of the apparatus of the special senses is more special, indicating to a degree the order of development of their several functions. The muscular sense extends to all the muscles; touch and temperature to the periphery, the end-organs residing largely in the skin. ${ }^{1}$ The other special senses, sight, hearing, taste, and smell, have each a particular locality: but they are grouped together, and their nerves, by reason of their special and closer connection with the central nervous masses in the skull, are called cranial nerves.

The Reacting or Motor Apparatus. The analogy between the receiving and the reacting apparatus is so close that they may be taken up together: more especially as the purest type of reaction, as will appear below, assumes that there is no break of continuity between them. The nature of the reaction itself is a point of function and is reserved; the apparatus is what asks attention now.

In the reaction we find another system of nerves, the motor courses, quite indistinguishable from the sensor courses, except in their localities and their endings. They are also alike among themselves as regards their end-organ, the muscles. ${ }^{2}$ They issue directly from the body of the muscles and converge to the spinal cord, of which they constitute roughly the anterior (front) or ventral portion-the so-called pyramidal tracts. The essential facts, again, are the continuity of structure throughout, and the universal distribution of the motor courses to the muscular tissue. The distribution, however, does not secure equally ready reaction of all the

1 And in the mucous membrane of the mouth and pharynx, which constitutes with the skin the derivatives of the epiblastic layer of the embryo.

2 The secretive and vaso-motor connections are, for our present purpose, neglected. 
muscles; indeed, some of the muscles are either entirely outside the range of voluntary control, or are brought within only by much exertion.

Ferrier holds that the lateral columns (the fibrous matter lying on each side between the dorsal and ventral columns) "contain the paths of sensation and voluntary motion." 'The most fruitful methods of study are, first, the "Wallerian" method, i.e., the observation of "degeneration" in tracts, in consequence of their separation from the centres from which they rise; and, second, the study of the embryological growth of the fibrous conductors and their connections. For example, after destruction of the motor areas of the cortex, degeneration of the so-called "pyramidal tracts" in the cord is distinctly evident.

At the upper end of the spinal cord there is an enlargement, the medulla oblongata, in which occurs a rearrangement of all the courses and their distribution to the various masses of the brain. Above the medulla again we find other white fibrous bodies-which need not be enumerated-serving two evident purposes; i.e., they gather together fibres which minister to the same function, and distribute these fibres to the cellular bodies at which such functions have their brain-seat. In these higher white masses, motor and sensor courses are inextricably interworen; and in only a few cases has research succeeded in establishing pathways up or down. Without giving details, we may say that the following points are quite definite.

1. Sensory tracts pass from all parts of the periphery of the body up through the (dorsal columns of the) spinal cord, cross (decussate) in part in the medulla, and reach the surface of the opposite hemisphere of the brain (largely the rear and nether portion). ${ }^{2}$

${ }^{1}$ Functions of the Brain, 2d ed., pp. 54-56.

' In this case and the following, the nuclear interruptions are not regarded: for details ef. Wundt, Phys. Psych., 3d ed., pp. 142 ff., and Foster, loc. cit., III. sects. 9 and 7. 
2. Motor tracts pass from all parts of the periphery up through the (ventral columns of the) spinal cord, cross in part in the medulla, and reach the opposite hemisphere in the motor zone (area on both sides the fissure: of Rolando, including the paracentral lobule ${ }^{1}$ ). These courses are called the pyramidal tracts from the pyramid form in which they are bunched on the ventral side of the medulla. See Figs. 4 and 5.

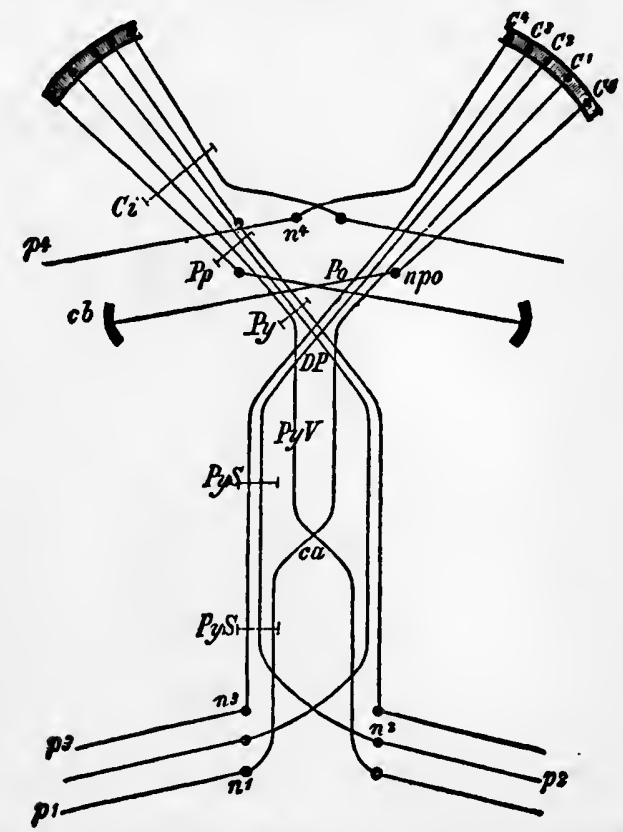
Fig. 4.-Scheme of pyramidal tracts $p^{1}, p^{2} p^{3}$ periphery of body; $n^{1}, n^{2}, n^{3}$.
spinal nuclei of origin; $P y S$, lateral pyramidal tract; $P y V$, anterior pyramidai tract; $c a$, anterior commissure of spinal cord; $D P$, decussation of pyramlds: $P y$, pyramids; $P p$, pes pedunculi cerebri; $C i$, internal capsule; $P o$, pons; $n p o$, nuclei pontis; $c b$, cerebellum; $p^{4}$, periphery supplied by cranial nerves; $n^{4}$, nucleus of origin of a cranial nerve; $C^{1}$ to $C^{5}$, cortex cerebrl (Obersteiner).

3. Association tracts develop, in the course of the life. of the individual, to connect all parts of the cortex of the brain with one another. They are almost, if not quite,

${ }^{1}$ See Senses and Intellect, Frontispiece and p. 114. 
absent at birth. In the words of Edinger: "They extend everywhere from convolution to convolution, connecting parts which lie near each other as well as those which are widely separated. They are developed when two

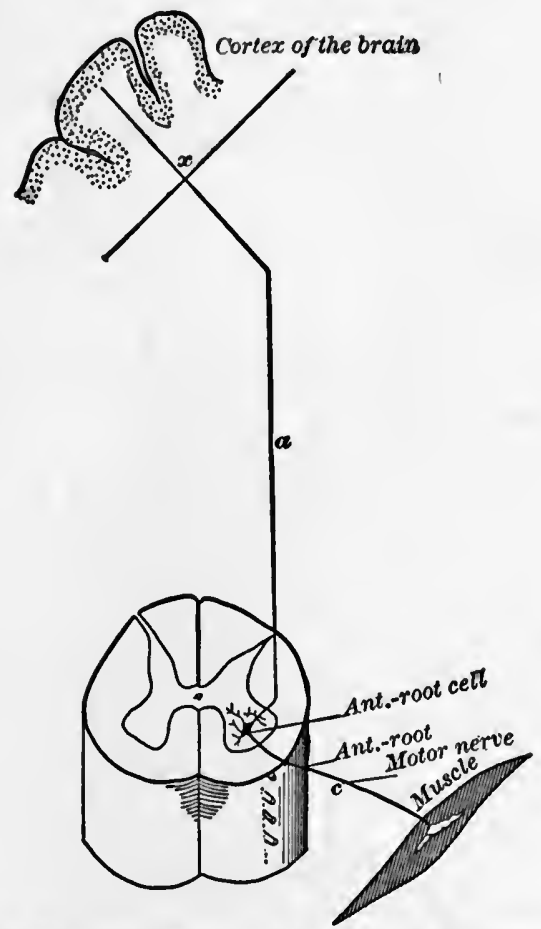

Fig. 5.-Diagram of innervation of a muscle. (After Edinger, Am. ed.)

different regions of the cortex are associated in a common action." ' See Fig. 6.

Under the same head may be included also the fibres which connect the two hemispheres with each other, making of them a single organ in relation to the lower parts of the system. Such connections are found in two great bundles called the corpus callosum, being the

${ }^{1}$ Structure of the Central Nervous System, p. 69. 


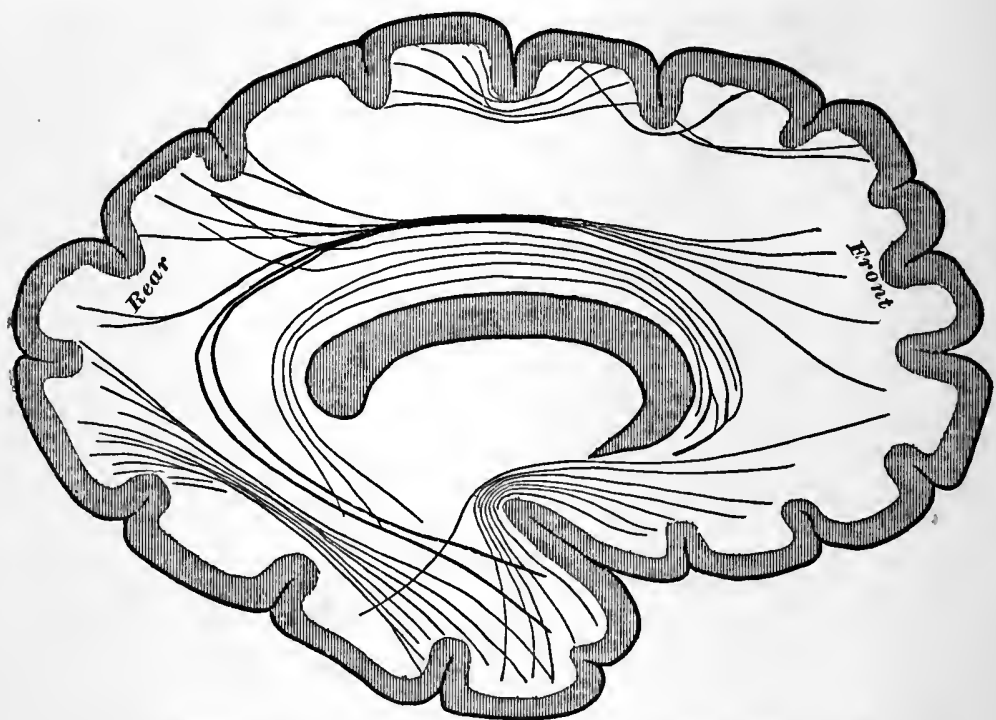

Fra. 6.-Diagrammatic representation of a part of the association fibres of one hemisphere. (After Edinger, Am. ed.)

floor of the great longitudinal fissure which separates the hemispheres from above, and the anterior commissure below. Both are shown in the accompanying figure (7).

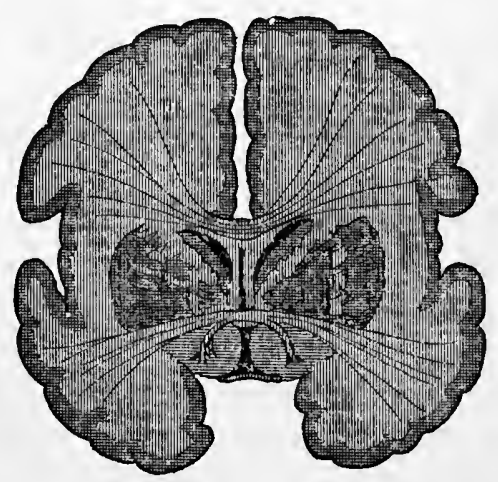

Fra, 7. Frontal section of the course of the corpus callosum and the anterior commissure. (After Edinger, $A m$. ed.) 
Tho Registering Apparatus. Under this term we include the more or less complex chain of cellular elements which constitutes the centre receiving and reacting. The word registering emphasizes again the integration or development side of the nerve-process. In its most general or schematic outline, the system is made up of two similar nerve-courses brought into organic connection at their upper end by this cellular series. It may be represented to the eye in the following simple way, $M$ being the motor course, $S$ the sensor course, and $C$ the central elements: the whole constitutes the elementary nervous arc (Fig 8).

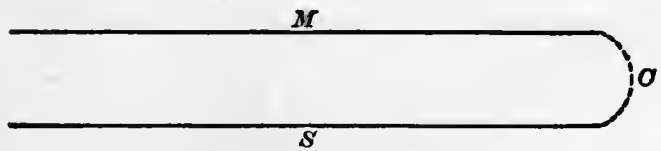

Fig. 8.-Nervous Arc.

Our knowledge of the central elements is exceedingly vague, both as regards structure and function. As to structure, the most exact thing that we can say is that the centre is cellular and probably in all cases complex. Its complexity is indeed so striking and elaborate that it is this feature that tends to obscure all others and render research fruitless. A general distinction is made by physiologists between the simple arc and the complex mass of many arcs with their accompanying highly integrated centre; but the simple arc is a pure abstraction. Indeed its very conception is dependent upon the results of an analysis of the centres which has never been made. In reality it is probable that the simplest nerve-reaction of which we have any knowledge involves a cellular mass and a number of alternative motor and sensor tracts. Such a relatively simple system is found in the ascidians, which have only a single ganglion with sensor and motor filaments. See Fig. 9. 
In distribution, the central masses again illustrate the hierarchical arrangement of a developmental order. The simplest of such ares are at the points of union of

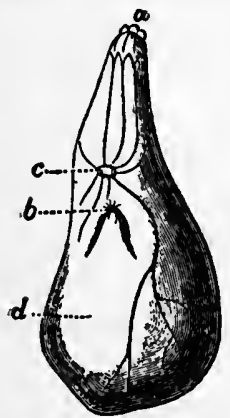

Fra. 9.-Nervous 'system of an Ascidian (Carpenter). $a$, the mouth; $b$, the vent; $c$, the ganglion; $d$, muscular sac. nerve-courses, points where the motor and the sensor find an interchange of energies, or a distribution so uninvolved as to follow from the nature of the nervous integration it represents, without appeal to a higher and more complex centre. Such comparatively simple points are called ganglia. For example, the nerves which enter the spinal cord on either side at intervals throughout its whole extent divide a short distance from the cord, and send branches called motor and sensory roots, respectively, into the cord. Just above the point of division, on the sensory root, we find a swelling or lump, a ganglion. The ganglion, it is thought, represents a junction, to use a railroad figure, for the transfer of passengers and the interchange of telegraphic messages. ${ }^{1}$ See Fig. 3 above.

The spinal cord is made up of a series of segments, together forming a column in the centre of which is a continuous mass of gray (cellular) matter. This gray column gives off the spinal nerves from its two posterior and two anterior horns (see Fig. 3): the nerves thus given off right and left at the same level meet just below the enlargement or ganglion outside the cord. Above the spinal cord the gray matter is enormously increased, as we should expect from the increase in the fibrous pathways already described. Thus a number of bodies are formed in three con-

${ }^{1}$ On the functions of the ganglia and centres generally, see below, $\S 2$. 
nected systems:' first, the most central gray matter, serving to connect the spinal column with the higher centres, and giving the cells from which arise the cranial nerves; second, the segmental system, including all the masses which lie in the interior of the brain (the most important being the striate bodies and the optic thalami); and third, the surface masses, the cerebrum, which has its gray matter arranged in layers, giving the cortex or rind, and the cerebellum or little brain, a similar mass behind and beneath the cerebrum with a similar cortex of its own. For present purposes the essential points again to be noted in this connection are, first, what we have called the hierarchical character of the series, the unbroken advance in structural complexity; and second, the continuity of connection and influence through it all.

This schematic and, from the physiological point of view, quite inadequate, description of the nervous system as an apparatus, will suffice for our psychological purpose. It aims to omit nothing essential to the estimation of current theories of the physical basis of sensibility. It also aims to present sufficient data for a clear understanding of the laws of function, as far as they are now known.

\section{§ 2: Functions of the Nervous Systey.}

II. The consideration of nervous structure leads at once to that of function. The word function points from anatomy to physiology, and precipitates us into a sea of contention among rival schools. Under whatever names they be discussed, however, a certain num-

1 Following Foster, Text-book of Physiology, pt. IIr. pp. 977-98. For details and diagrams see any of the Physiologies; Ladd's small Outlines of Physiological Psychology is couvenient for reference. 
ber of processes are recognized by contemporary physiologists as being distinctly nervous. It is well here, as in the treatment of the question of structure, to proceed synthetically, endeavoring first to present the original properties of nerve-substance.

Fundamental Properties of Nervous Tissue. Experimental research upon living nerve-tissue has issued in a conception of protoplasm which includes two functional elements. At the first glance, nerve substance exhibits the property called in general scientific nomenclature irritability. This property is by no means confined to developed nerve-elements: it is exhibited by all living animal tissue, by forms of organism in which a nervous system is entirely wanting. ${ }^{1}$ In some forms of vegetable life, as the sensitive plant, the same property is presented. In the case of nervous irritability, however, whenever the substance assumes the complexity of a system, we are led to view it under two distinct functional rubrics. Recalling a former division, we find the receiving and the reacting apparatus to be appropriate to the same function, that of propagation, transmission, or conduction; and the central arc, the registering apparatus, suggests a function of integration. Assuming the results of later exposition, these two functions may be called respectively Neurility and Sentience.

Let us consider, for example, the element $C$, of Fig. 8, above, to be the centre or nucleus of a protoplasmic mass, and the two lines $M$ and $S$ to be two radii from the centre to the outer surface. If, then, the mass

1 These primitive unicellular organisms show a remarkable sensitiveness to differences of light and darkness. See the reports of Engelmann, Pflüger's Archiv, xxIx, quoted by Romines, who gives a collection of facts regarding the sensations of the lower invertebrates, Men. Evolution in Animals, pp. 80 ff. 
be stimulated at the outer end of $S$, and this be followed by the withdrawal of the point stimulated, we have a phenomenon of irritability. But we may suppose $S$ to be a line of conduction of the excitation to $C$, and $M$ the line of reverse conduction or reaction which results in the contraction: both of these fall under the conception of Neurility. The process by which they are held together at the exchange-bureau $C$, so to speak, is Sentience. This rough conception may be made more distinct as the two processes are taken up singly.

1. Neurility. Under the head of neurility we are introduced to a class of phenomena which have striking analogies in physical science. The conception or phenomenon of conduction is familiar in what we know of light, sound, and heat propagation; but the special analogy which at once suggests itself is electric conduction along a metallic wire. Setting aside, as a matter of speculation, the hypothesis that nervous force is identical with electricity, we may still find in the analogy much help to a clear conception of nervous conduction.

Indeed the theory of nervous action most current among authorities-as well as in the popular mindfinds its general exposition in terms of the analogy with electric action. On this theory, the nerve-courses are simply and only conductive tracts, as the electric wire in a telegraph system : the centres, on the contrary, are the generators of "nervous force." At the centre we have, therefore, a storage-battery from which force is drawn off along the motor courses upon the occasion of the arrival of a stimulus from the sensor course. The centres, on this theory, are the essential nervous agents or producers, and the courses are brought into operation only as they are charged from the central battery or pile. Neurility, therefore, is simply the molecular 
state which constitutes a course a good nerrous conductor.

This theory is objected to both on theoretical grounds and from experiment. It makes the distinction between courses and centres too absolute and mechanical. According to it, any distinct dynamic property is taken from the nerve-tracts; while experiments show that the elementary portions of both sensor and motor nerves have a life and function of their own. The eye when removed from its socket, thus losing all connection with a centre or ganglion, still shows sensitiveness to light, and has a motor reaction in the contraction. or expansion of the iris. ${ }^{1}$ Pflüger maintains that there is an increase in intensity in the nervous disturbance as it traverses the motor nerve, and Richet ${ }^{2}$ thinks a similar increase in the sensory nerves probable.

Accordingly, another theory is advanced which seems more philosophical to the present writer, as far as he - ventures to have an opinion on a matter so purely physiological. This second conception of the nervous system makes it a living organism instinct with nervous force or neural properties throughout. This system is in a state of unstable equilibrium and constant change, due to stimuli through sense-organs and to spontaneous central discharge. Disturbances tend to equalize themselves everywhere in the system by a species of centrifugal and centripetal tension, which, through its greater or less effectiveness in this direction or that, upon this course or that, results in conduction or neurility. Differentiation,

1 Brown-Séquard, Proc. Roy. Soc., 1886, p. 234. See note of many other such facts in Lewes, Phys. Basis of Mind, p. $231 \mathrm{f}$. Another interpretation may be given such facts, i.e., that the stimulus acts immediately upon the muscle : but this would not damage the position in the text, since it is probable that nervous and muscular irritability do not differ in kind. Cf. Kühne, Croonian Lect., Proc. Roy. Soc., xLIV. pp. $433 \mathrm{ff}$.

${ }^{2}$ Recherches expérimentales et cliniques sur la sensibilité, p. 297. 
therefore, in the system is primarily structural differentiation, due to the adaptation of the life-process to changing conditions in the environment.

Mr. Lewes ' argues for the second interpretation with great clearness and force, and Dr. Broadbent has recently stated it concisely before the Neurological Society of London. ${ }^{2}$ The term neurility is also due to Mr. Lewes.

The "dynamic" conception, as the latter may be called, is supported by a class of facts which show a ready and facile interchange of influence throughout the system difficult to account for if the parts between which the transfer occurs are functionally distinct: such general transfer affords the so-called law of diffusion." For example, a single sensory stimulus may, when intense, or when the system is excited from disease, lead to general irritation and diffusive discharge. On the other hand, a reflex having its centre in a particular spinal ganglion may be partially stopped by a sensory excitation from another part of the body." Cases of association between sounds and colors, and phenomena of contrast $^{\circ}$ generally, show such dynamic connections between disparate sense-regions. Urbantschitsch ${ }^{7}$ found that the perception of color was improved when a tuning-fork was made to vibrate near the ear.

' Physical Basis of Mind, Problem II.

See Brain, vol. xr. p. 396. For profound considerations looking to the electrical interpretation, see Kahne, Croonian Lect., Proc. Roy. Soc., xuIv. pp. $427 \mathrm{ff}$.

${ }^{3}$ Bain. This law is considered more fully later, in connection with the subject of "emotional expression," Chap. X. $\S 1$.

4 Wundt, Phys. Psych., 3d ed., I. p. 182, and Foster, loc. cit., § 593 ; also see Lewes' collection of striking facts in Phys. Basis of Mind, p. 334.

S See Senses and Intellect, p. 208.

- Below, Chap. IV. $\$ 1$.

- See this and many other facts proving the general position, Pflitger's Archiv, xuIr. p. 154. James (Psychology, 1. pp. 374-82) gives some striking fllustrations. 
Whichever of these views we may adopt, the result is the same for the consideration of the fundamental nerveprocess. If we hold that neurility is simple molecular conduction, then it is extra-neural and the term neurility is a misnomer ; that is, the central process alone is left. On the other hand, if we hold that the system is functionally one throughout, then we extend the activity of the centres in kind also to the elements of the courses and central action is again the only action. In other words, we are shut up in either case to what we have called sentience for our ultimate point of departure. From the latter point of view, the amount and extent of "diffusion" would indicate likelihood of consciousuess and its intensity, the organic reactions where diffusion is least being outside of the personal consciousness.

However it may be explained, nervous conduction is of fundamental importance for the theory of sensibility. And for practical purposes the wave or current theory serves, as in electricity, all ordinary requirements. The nervous wave, therefore, is called centripetal or afferent when moving toward the centre, and centrifugal or efferent when moving toward the periphery. The rate of transmission differs somewhat in the two directions, being about 120 feet per second for sensor and 110 feet for motor impulses. Transmission through the spinal cord takes place considerably more slowly.

2. Sentience. From the foregoing it seems that our search for knowledge of "nervous force" has at last taken the right turn, and in "sentience" we are to have our curiosity satisfied. But here it is even more emphatically true than anywhere else that neurology has nothing final to offer us. As to what goes on when a centre receives, registers, and reacts upon a stimulus we

- are completely in the dark. From the interpretation of results, and from physiological analogies, some general 
statements may be made, and these general statements are valuable for psychology; but they do not pretend to throw any light upon the genesis or nature of nervous force.

a. Integration. Of these general statements, the first concerns what has already been called the integrating function of nerve-centres. By this is meant the building up of a centre to greater complexity of structure through new stimulations. It takes place by reason of the extreme plasticity of the nervous elements in taking on arrangements suited to more habitual and, at the same time, more complex reactions. The centre becomes the theatre of multiple and conflicting stimulations; its reaction is the outcome of a warfare of interests, and the pathway of discharge is a line of conduction most favorable to future similar outbursts. A centre gains by such complex activities in two ways : first, its habitual reactions become a rock-bed or layer of elements, so to speak, of fixed function issuing in established paths of least resistance; and second, the centre grows, gaining new and more mobile elements, and responding to more complex and difficult motor intuitions. For example, the centre for the movements of the hands is educated from the early painful lessons of the baby's finger-movements to the delicate and rapid touch of the skilled musician. Not only has the centre become fixed and automatic for movements at first painfully learned, but it has become educated by learning, so that it acquires new combinations more easily. This twofold growth becomes the basis of the division of the sentient apparatus into centres and ganglia. The "rock-bed" elements, so called, fall into fixed ganglionic connections, and the new and free cells take up the higher function, only in their turn to become "fixed" by habit and to give place to yet other and more complex combinations. This integrating process is what gives the hierarchical order to the sys- 
tem, and throws its law of development into fine relief. Integration, therefore, represents a structural change in the direction both of simplicity and of complexity: of simplicity, because it gives ease and rapidity to habitual movements ; of complexity, because it brings into play new elements which must be assimilated to the unity of the centre.

The influence of habit will be the subject of further remark below. It is sufficient to say that the law of habit simply holds up to view one side of this process of integration. The more perfect and smooth a muscular reaction, the better its elements may be said to be integrated in a common function; and habit, which means repetition, is the means of securing this result.

As to the range of such education of the centres in both directions-the performance of simple automatic and reflex movements, and readiness to acquire new complex systems of morement-there seems to be no practical limit. The voluntary passes into the automatic, gaining in spontaneity and precision, as in the movements of walking, running, etc.; and on this basis, the voluntary again builds up surprising feats of muscular combination and skill, as in the supple and graceful performances of the athlete and the gymnast. Our whole active physical life illustrates the interplay of these two tendencies.

This principle of integration covers, in its two aspects, the law of growth of living tissue in general. Exercise always tends both to consolidate and to enlarge an organ. A muscle becomes more ready and exact, as well as larger and more capable, with frequent use; and the same application has been made of the principle to mental functions, notably to the memory. The striking peculiarity of the case in regard to nervous activities is the excessively detailed differentiations it works : we have here not only the rise of new centres from old ones, but organic pathways developed between them, and a progressive advance secured throughout the system, from the spinal ganglia up to the cerebral cortex.

$b$. Retention. The conception of integration necessarily includes that of the permanence of the modification on which it depends. If reactions are integrated in such a way as to secure the upbuilding of the system 
and its more perfect adaptation, then we must suppose that each reaction works a minute structural change in the organism. So much is included in the conception of integration. And from the physiological side this would seem to be sufficient. Retention as a physiological principle may, therefore, be called growth in functional complexity; while the term integration refers rather to growth in structural complexity.

Accordingly, the conception of nervous retention runs somewhat like this : Nervous retention is a state of dynamic tension or tendency due to former nervous discharges in the same direction; the two essential points, again, being the dynamic or tension aspect of nerve-action in general, and the particularization of this tension along a given path determined by previous like discharges.

c. Selection. A third fact of sentience may be called selection. It denotes the undoubted property of the living nervous system of reacting within limits of greater or less adaptation. It shows preference for certain stimuli above others, if the word preference can be shorn of all its reference to conscious choice. A system will react on a stimulus at one time which it will refuse under other circumstances: or it will distinguish between stimuli exactly alike, as far as human sensibility for differences can determine. The brainless carp will distinguish food with some degree of precision, and experiments by Pflüger and Göltz ${ }^{1}$ on brainless frogs show that they adapt their muscular reactions to varied positions of the limbs which could not have been experienced before in the life of the creatures. Schrader has also reported many similar cases of apparent preference and choice in brainless pigeons. ${ }^{2}$

1 Functionen der Nervencentren des Frosches, 1869. Cf. Ferrier, Functions of the Brain, 2d ed., pp. 73-r6 and 109.

'Comptes Rendus, vol. 102. Lewes gives interesting general cases, Problems of Life and Mind, 3d series, I. pp. $42 \mathrm{f}$. 
Such instances seem to show a selective function in nerve-reactions of the purest type, i.e., those simply ganglionic, where the effects of consciousness are either quite wanting or reduced to a minimum in intensity. If we were to take them up in detail, we would find that we could distinguish two forms of what appears to be selection, an immediate form and a derived form. Under immediate selection may be included all cases in which a single stimulus is sufficient to call forth the preference; under derived, cases in which the preference is shown after one or more mistaken and hurtful reactions.

Now, derived selection finds its explanation in the integrating function already spoken of. The effects of mistaken reactions are inhibitive rather than stimulative, by the same law which in higher organisms connects pleasure and pain with good or damage to the system. Hence stimuli of this character work the conditions in the system which lead to their own refusal. It is certainly, at any rate, a case of remarkably quick and happy adaptation.

In cases of original selection, the explanation is probably to be found in the peculiar delicacy of the receiving apparatus. ${ }^{2}$ To say that a brainless animal selects when we are unable to point out differences, is only to say that more debased currency will pass for gold with us than with it. Instead of selecting between two stimuli, therefore, it has had only one, and has responded to it: the other being mistakenly considered by us as fitted to excite it. Does the nervous system select from a multitude of similar touches? The magnet selects from a multitude of similar filings: and the explanation seems to be the same. Neither the touches nor the filings are similar after all.

1 What Ferrier calls "more complex and special afferent and efferent reactions," loc. cit., p. 118. 
Another explanation of the latter kind of selection must be mentioned, both because it is held and becanse it affords. a philosophical and quite plausible hypothesis: it is possible that our subsequent discussions will bring us into accord with it. It holds that sentience involves consciousness, that nervous action is always conscious (not self-conscious) action, and that a fundamental mark of consciousness is preferential selection or choice. Below ${ }^{1}$ the general relation of consciousness to sentience is discussed, and occasion will be seized to revert to this theory. The explanation given to nervous selection has psychological significance, since, according as it is explained, it may or may not give us data for our theory of voluntary choice.

Physiologically the process of selection may be pictured somewhat thus: Given a state of nervous tension, with a possible outlet in forward movements: this outlet as an alternative is supported by various traces of former forward movements and their consequences. A pair of stimuli are received, say, by weak optical. stimulation of objects resembling flies, in the case of the brainless carp : a tension is created toward the flies; that is, a tendency to move forward. The tension is stronger toward the real fly by reason of its greater appropriateness as a stimulus. This stronger tension is reinforced by memories of juicy flies already eaten. And lo, the selection is made, the forward movements follow!

The further discussion of this question involves considerations of such breadth and philosophical importance that it is of necessity postponed. It may be remarked here, however, that if the mechanical conception with which we started is to hold throughout as a sufficient explanation of nerve-reactions, we must hold either that there is no true selection, the first. explanation given above (in the large print), or that consciousness is always present, and with consciousness the selective function is added. It is essential to note that the second alternative (the explanation given above in the finer print) is not necessarily inconsistent with the mechanical conception of the system. The law of action and reaction, of conservation 
of force, may hold even though we find a directive and selective agency in consciousness. A compound commutator in a telegraph-office does not modify the strength of the electric current : but it does convey the selective agency of the operator along one connection rather than another. Consequently, if we find evidence of such selective agency later in the will, the mechanical conception of the nervous system will not stand in the way of our accepting it. It is important to note this, since the claim that consciousness is an "epiphenomenon," a piece of unessential by-play, rests upon the supposed implications of the mechanical theory. ${ }^{1}$

Law of Nervous Dynamogenesis. Sentience, in view of what has now been said, is a general word for the rise and distribution of nervous force. The receiving and reacting functions are both essential, the one necessarily giving rise to the other: there is no incoming nervous process, therefore, that does not tend to liberate energy on the outgoing courses. Every stimulus has a dynamogenetic or motor force-may accordingly pass as a statement of the law in its individual bearing, the only bearing which is available as having a psychological analogy.

\section{§ 3. Kinds of Nervous Reaction.}

The twofold growth of the nervous system spoken of under Integration gives us data for a distinction among different reactions. Integration involves, on one side, a downward or "ganglionic" growth, represented in function by the more unconscious and unintended reactions of the muscular system; and, on the other side, an upward or "central" growth, represented by the more difficult muscular performances, in which attention and effort are called out. These two laws of growth act together, and in the result, in our motor experience, we

1 See below, Chap. XV. $\$ 2$, where the implications of a selective theory of consciousness are fully stated. 
find every degree of nervous facility or the contrary. Three stages of such growth, from down up, so to speak, are usually distinguished.

1. Automatic Reaction. By the automatic in nervefunction is meant the self-acting, i.e., those reactions which find their stimulus in the living conditions of the physical organism itself. Certain organic processes are necessary to the life of the individual and the race-circulation, respiration, digestion, reproduction. The dependence of these essential functions upon external stimuli of time and place would give an accidental and varied character to these reactions which would subserve death rather than life. Accordingly, the automatic centres represent the most consolidated and fixed portions of the nervous system, at the same time that they are complex and elaborate. These functions may or may not be conscious, their most healthful activity being generally most free from conscious oversight. With very rare exceptions, ${ }^{1}$ also, they cannot be modified by the will or brought under voluntary control.

Automatic action may be said to represent the goal and ideal of our nervous development considered as a mechanism. Its action is self-stimulated, in the sense that external compounds are taken up into the life-process in such a way as to prepare the necessary stimuli for its own continuance. The dependence upon external conditions is accordingly made more and more remote. The life of a child depends upon the regular presence of a single food of few properties. But the system develops to the capacity for variety exhibited by the soldier or athlete. This mechanical ideal, however, is a distinctly lower ideal than that which is presented in the "central" growth; for there the organism is preparing for reactions by which remote mechanical conditions in the environment are not only reacted to, but foreseen, modified and controlled. The automatic functions have their nervous seat largely in the basal ganglia beneath the brain and in the cord.

1 Cases of voluntary control of the beating of the heart. 
2. Reflex Reaction. A nervous circuit is reflex when its motor reaction upon a particular kind of stimulus is single, definite, constant, and does not involve volition in its execution. In more general terms, a reaction is reflex whenever we are certain beforehand that it will take the form of a particular well-defined muscular movement, and will do its work without any interference or mandate from ourselves. We are disposed to stand apart and attribute the reaction to the organism or to the external stimulus. For example, if a ball suddenly approach my eye, it closes, or if it strike sharply upon my knee, my foot flies up : ' we do not say $I$ close my eye or raise my foot. Or we go farther out still and say the man who threw the ball made my eye close or my foot fly up-so thoroughly do we distinguish this class of reactions in consciousness from those which we attribute to our own agency.

In its physiological character, this kind of reaction represents a less organized and consolidated system of elements than the automatic. A reflex reaction is generally conscious in its operation, and always so in its completed results. Its centre, also, is not cut off functionally from the higher centres of the brain, which exercise a controlling influence. Yet we know that this connection is not an essential one to the reaction itself, since after the removal of the cerebrum and with it all active consciousness (certainly; perhaps all consciousness), the reaction still takes place. Each of the segments of the spinal cord has its own reactions apart from its brain-connection." Indeed, reflex reactions are most perfect and pure when consciousness in the form of attention is not directed to the movements. These facts tend to throw

1 For example, without assuming with Foster that the knee-jerk is a true reflex. XXVIII.

${ }^{2}$ Ward's experiments on the Crayfish, Proc. Roy. Soc. Eng., vol. 
reflexes rather on the side of the "downward" growth spoken of, and assimilate them to automatic reactions. The well-known phenomena presented by the reactions of a brainless frog illustrate pure reflexes very clearly.

The downward growth appears from the fact that many of our reflexes are acquired from habit and repetition. Motor processes at first difficult and simple are welded together in complex masses, and the whole becomes spontaneous and reflex. The case is cited of a musician who was seized with an epileptic attack in the midst of an orchestral performance, and continued to play the measure quite correctly while in a state of apparently complete unconsciousness. ${ }^{2}$ This is only an exaggerated case of our common experience in walking, writing, etc. It represents, from the standpoint of body, the motor organization in consciousness already pointed out under the head of " motor intuition." s Just as a number of single experiences of movement become merged in a single idea of the whole, and the impulse to begin the combination is sufficient to secure the performance of all the details, so single elementary nervous reactions become integrated in a compound reflex.

This consideration leads to a further distinction between more or less organized reflexes ; namely, between what are called secondary-automatic reactions and reflexes proper. In the case of our movements in walking, for example, the successive reactions are not sufficiently organized to belong properly to a single stimulus-say the original idea of our destiuation, or the sensation

1 The mechanical uature of such reactions is admirably shown by the globular sea-urcbin: "If two equal stimuli be applied at ny two points of the globe. the direction of escape (of the creature) will be the diagonal between them; if a number of points be simultaneously irritated, one effect neutralizes the other, and the animal rotates upon its vertical axis."-Romanes, Mental Evolution in Animals, p. 29.

"Trousseuu, quoted by Lewes, Pliys. Basis of Mind, p. 221.

Senses and Intellect, chap. viı. § 3. 
of our first footfall upon the pavement; but the steps in succession are probably excited by the successive afferent impressions of the steps accomplished. Each step stimulates the next, etc. That there is no voluntary stimulation after the first is seen in cases of revery or absent-mindedness, when we go along accustomed paths and find ourselves where we least intended to "bring up." The distinction, therefore, is merely one in degree of integration. If the centres are sufficiently organized "downwards" to carry out the entire chain of movements when once begun, we have a pure reflex; if new sensory stimulation is necessary at each stage in the series, the reaction is secondary-automatic.

This distinction was first pointed out, it is thought, by Carpenter," to whom the expression "secondary-automatic" is due. It is a real distinction, but under his terminologywhich we follow simply to avoid unnecessary novelty in the use of terms-the essential idea is obscured. The term secondaryautomatic assimilates such reactions to the automatic or essential processes of the organism. Now, the latter have regular rhythmic action, a unity of successive stages, and a unity of end that is quite wanting in the so-called secondary-automatic. The latter, on the contrary, as far as they differ from a series of simple reflexes, do so in calling out a more or less coördinated system of stimuli. For example, in the walking case, the eye guides the movements in turning corners and in identifying familiar objects, the ear takes in accustomed sounds, the foot notes the material trod upon, etc. In short, the stimulus is a coördination of general sense-conditions, and such a coördination is necessary to the continued reaction. If a new sound break in on my ear, if my foot come upon a board walk instead of flag-stone, or if a strange building suddenly confront me, I am "brought to myself" at once, and the reaction is stopped.

We would, therefore, substitute the expression " coördinating" reflexes for secondary-automatic, and contrast them with " simple" reflexes. As this coördination is represented in the centre by different sensor elements, and as these elements thus become drawn into a common motor discharge, the re-

${ }^{1}$ Human Physiology, Philadelphia, p. 504 f. 
action called coördinating is simply a stage in the "upward" growth included under integration.'

3. Voluntary Reaction. A third great class of nervous reactions is called voluntary. By voluntary reactious is meant such motor effects as follow upon the conscious will to move. They cover the whole class of intended movements and those brought about by greater or less effort. Voluntary movements show variation in several distinct particulars; such as strength, continuance, rapidity, and direction. "The strength of the movement is regulated by the strength of the initial motor impulse; the extent depends in reality upon the same influence; the rapidity results from the more or less rapid succession of voluntary (motor) impulses; and the direction is determined by the voluntary localization of the incitation upon certain groups of muscles." "

The voluntary reaction undoubtedly represents the highest stage of development of nerve-tissue as respects complexity, as well as the lowest stage as respects consolidation and fixedness. It is the polar opposite of the purely automatic function. The nervous elements are in a state of extreme mobility and instability. The connections through its mass are infinite in number and complexity, and numberless alternative courses are accordingly open to the motor outburst of a sense-stimulation. Considering the state of the cerebral centre dynamically, we may say that its potential energy is constantly seeking discharge, and that this discharge in one course rather than another-the course pictured and designed in consciousness-represents the line of tension which prevails.

The last expression, though psychological, is neces-

${ }^{1}$ Professor James has propounded an interesting theory of the nervous process of these serial reactions, Principles of Psychology, II. pp. 572-92.

- Jaccoud, quoted by Bastian, Brain, x. p. 52. 
sary to express the physiological fact which distinguishes such reactions. The stimulus is in all voluntary reactions a central one, and a conscious pictured one: this much at least. Now the point of interest here is : what is the meaning of central in this connection? If we admit that no discharge from the centres can take place without a previous liberation of tension, then we may divide such liberations from tension into two classes: that which is brought about by an incoming current, and that which is brought about by an earlier cerebral discharge. The former is a reflex reaction, the latter may be a voluntary reaction. One at least of the conditions of voluntary action is fulfilled, the physiological condition. Whether this is sufficient in all cases, or in any case, to account for the reaction, it is our subsequent task to determine. Here it is clear, if the conception of nervous function urged above is at all correct, that the process, as far as it is physiological, is exactly the same as in reflex reactions, except that the elements form a more complex and less solidified system, and that the physiological condition of their activity is a previous state of central nervous discharge in adjacent or functionally connected centres. This essential sameness of function, with partial differentiation of organic seat, is confirmed by experiments showing that the tracts of the anterior root-zones, while transmitting motor impulses, are yet motor in a sense different from the pyramidal tracts. They appear to be "paths of conduction of motor impulses from centres distinct from those of voluntary motion proper." Voluntary impulses are carried out through the same apparatus as involuntary, but have their initiation in the cerebral centres. ${ }^{\prime}$

${ }^{1}$ Ferrier, loc. cit., p. 57. See also the experiments noticed below (Chap. XVI. §5), aiming to show that reactions similar to those usually voluntary may be produced by artificial means. 
The discussion of voluntary reactions is to such a degree involved with the discussion of conscious volition that it is well to postpone any further description of their character. 'The general terms of the text serve to mark them off from the foregoing nervous processes.

4. Negative Reaction or Inhibition. Under the name of inhibition, or arrest,' a class of phenomena is included which are, as far as our knowledge goes, peculiar to nervous activities. Every positive reaction is accompanied by a reverse wave, an arrest, so to speak, of its full effects. It is analogous to a negative force acting to counteract and neutralize the outgoing discharge. It seems to take place in the centre. The effective force of a reaction, therefore, is always less by the amount of nervous arrest. This neutralizing factor has been measured in certain conditions of nerve-reaction."

An accepted explanation of arrest is still a desideratum in physiology. Theories which go no further than to say that it is negative reaction, or an inhibitive property of nerve-tissue, or native resistance to conduction in the gray matter, ${ }^{3}$ are only verbal descriptions. No explanation shall be attempted here certainly : the writer's aim is merely to bring the phenomenon within the general law of nerve-development already advocated. It will be enough to show that inhibition is a necessary factor from the first in the development of the system, and has adequate recognition under the twofold law of integration.

The general conditions of nervous arrest may be described in more detail, and for purposes of later interpretation, in formal numerical order :

(a) The kind of reaction showing least arrest is the reflex; and, in general, the more consolidated' a nerve-

1 German, Hemmung.

See Wundt, Mechanik d. Nerven, I ; also Phys. Psych., chap. vI. 2.

3 Meynert.

4Cf. Wundt, Phys. Prych., chap. v1. 3 : he uses the word ersehopft ("formed") with the same meaning. 
track or centre, the less exhibition do we discover of the reverse wave. This would seem to indicate that inhibition is not a phenomenon attaching to "paths of least resistance," and does not belong on the side of so-called "downward" growth.

(b) Inhibition is at its maximum in reactions which involve centres of most complex activity. The phenomena of voluntary control-inhibition by the will-are in evidence here, however we may construe the will. For it should be remembered that we must find a mechanical basis for muscular control even though we advocate a directive and selective function of will.

(c) Hence inhibition is a concomitant of instability and complexity of nervous tissue : and it belongs on the side of the "upward" growth of the system.

An interpretation of these facts may be suggested in the light of what has already been said. We find the growth of the nerrous system to be by a twofold process of adaptation. Now this adaptation means the taking up into itself more and more of the mechanical conditions of its environment. The protection of sight requires a shield over the eye at moments of exposure : the system gets to itself a reflex function of closing the lid with no voluntary impulse or even against such an impulse. The system has adapted itself mechanically to the environment. Now external resistance as a mechanical condition is in like manner carried over into the system and becomes also a protective property. It seems to serve the part outside of consciousness which pain serves inside-a "governor" or regulator, neutralizing and equalizing the spontaneities of life. The function becomes still higher, outside as well as inside of consciousness, when volition dawns, since one of its most essential uses is the higher inhibition which is called control.

There is, therefore, a development in the conception 
of inhibition corresponding throughout to the structural development of the system in complexity and instability. This is what comes out, moreover, when we consider the dynamic aspect of the case. Inhibition, then, is the presence of an opposing tension, and is possible in proportion as the system grows away from a single line of action and reaction toward a complex interplay of force-pressures. The liability of any particular muscu'lar reaction to arrest would be expected to vary, as it does, with the number of possible alternative reactions, and the delicacy of their balance over against one another.'

This general view is also sustained by the fact now established that each segmental reflex in the spinal cord is subject to inhibition from the higher segments, and in turn inhibits those lower down. The reflexes of a frog's legs immersed in dilute acid are more rapid and violent after the hemispheres have been removed-showing the normal inhibitive function of the cortex ${ }^{2}$ : and the reflexes of a lizard's tail have been shown to increase in vigor as the segments of the spinal cord are successively removed.' The same truth is made plain from the fact that lesions of the motor zone of the cortex in man porduce greater motor disturbances than in animals, and greater in the dog than in the rabbit; the inference being that the subcortical centres are more independent, less inhibited, as we go lower down in the scale of animal organization.

${ }^{1}$ On voluntary inhibition, see Chap. XV. $\$ 1$.

Wundt, loc. cit., 3d ed., I. pp. 174-182, and Foster, loc. cil., $\S 593$.

Schiff, Lohrbuch d. Physiologie, p. 200. The same lack of inhibition appears in the greater automatism, suggestibility, and wayward im. pulsiveness of certain forms of insanity. 


\section{§4. Corolliaries: So-Called "Principles of Nervous Action."}

The foregoing discussion has brought us to a position from which to estimate the current " principles of nervous action." That they are corollaries deducible from the more particular truths already enunciated is in itself proof of the truth of the conception sketched in the foregoing pages. These "principles" may be spoken of in their logical order.

I. Principle of Specialization of Function. According to this principle, different regions of the nervous system are concerned with different and exclusive functions. It applies to the functions of the special senses considered as reporting qualitatively different classes of sensations; to the sympathetic as contrasted with the sensori-motor system ; to the ganglionic centres as controlling the movements of specific groups of muscles ; to sensory as distinguished from motor elements; to the special areas of the cerebral cortex which experiments have led us to believe have distinct motor, sensor, and perhaps intellectual and volitional functions. It is only a further extension of the more fundamental principle of integration which has been already considered; for the two growths " upward" and "downward" lead to functional divisions between the parts thus developed. The nerves of the leg, for example, as their simpler actions become more reflex, have their central elements in ganglia which are thus consolidated into an organ of special function; and the higher centres, being thus relieved, are given over to other and more complex coördinations. In short, a "specialization" of functions has taken place.

Most important consequences flow from this principle in the sphere of brain physiology and anatomy. And in the topographical divisions of the cerebral surface 
we find facts highly important to our own science. The most exact determinations of particular areas in the cortex have been made for the different gromps of muscular movements, ${ }^{1}$ the "motor zone" comprising homologous areas in the two great lateral halves of the brain. The centres for the special senses are-less exactly determined; but it is probable that in the near future our knowledge will be extended to such a degree that all the phenomena of the simple sensor and motor consciousness will have well-determined anatomical representation in the brain and cerebral ganglia.

One of the most difficult and important questions yet remaining open is the determination of the particular regions which contribute to consciousness, i.e., which are sensory. Are we conscious of the motor centres at all, or is all our consciousness of movement, as well as other sensibility, mediated by elements which are only sensory? And may sensory areas be also motor? 'That motor and sensory functions may at least be performed by the same areas, is shown by Shäfer ${ }^{2}$ and Munk, who find (in opposition to Ferrier) definite movements of the eyes following electrical stimulation of the sight (sensory) centre (occipital lobe) in dogs and monkeys." It is possible (Munk) that this centre controls reflex eye-movements, and that the eye-morement centre in the Rolandic region is the seat of voluntary morements. This view agrees with the suggestion of Bianchi " that the ordinary stimulation of the motor areas does not pass directly out to the nerves, but passes first throngh the sensory centres: a position supported by all cases of sensory effects following the stimulation of motor areas. Beannis holds that the motor elements have an immediate element of conscionsness. The possible absence

1 See Senses and Intellect, frontispiece, and p. 114.

2 Proc. Roy. Soc., vol. 43.

s See Amer. Jour. of Psych., III. p. 373.

- Schafer is led to the position that the visual area represents in some detail a projection of the retina upon the cortex. This would seem to give countenance to the view (Lotze) that the "local signs" for the retina (cf. Senses and Intellect, chap. virr. §4) are relative degrees of muscular innervation: since this projection is an arrangement of motor functions.

' Quoted by Donaldson, Amer. Jour. Psych., III. p. 551. 
of consciousness in the case of reflex reactions performed by the cord may be accounted for by the recent discovery claimed by Kölliker, that there are in the cord no sensory cells (i.e., cells having processes continuous with incoming sensory nerves). ${ }^{1}$

The phenomena of the apperceptive consciousness, being complex and coördinative, present analogies which would lead to the view that their physiological basis consists largely in connections among these definite areas, i.e., by the associative fibres. Yet the hypothesis of centres for such coördinations, points of convergence of intracentral connections, is gaining prominence and anatomical support; the centre for the coördination of movements being - or at least involving - the cerebellum, and the intellectual operations having their representation in the frontal lobes."

Facts of Specialization. A general fact or two may be mentioned in view of subsequent points of discussion. In the two halves or hemispheres of the brain we are led to recognize a twofold or duplicate organ, analogous to the doubleness of the eyes while performing together a single function. In regard to the function of the brain as a whole, we may say that in the main it is performed equally well by either hemisphere alone. If one hemisphere be entirely removed or destroyed, there is no perceptible impairment of the mind, at least in its great apperceptive activities. The hemispheres are moreover capable of separate activities at the same time: the movements of organs on the right side of the body which are governed by the motor area in the left hemisphere may be different from simultaneous movements

${ }^{1}$ Zeitschrift für voiss. Zoologie, Dec. 1890. See résumé by Donaldson, Ainer. Jour. Psych., III. p. 548.

${ }^{2}$ Wundt, Phys. Psych., 3d ed., I. pp. 210-13.

3 Ferrier, loc. cit. 
on the left side governed by the motor area in the right hemisphere. Again, there are certain functions which are presided over by one of the hemispheres exclusively, the other having no part in them: the motor speechcentre is in the left hemisphere for right-handed persons, and it is probable that there is a corresponding functional development for the delicate movements of one hand only, as in writing, etc. Accordingly, instead of considering the brain with Brown-Séquard ${ }^{\prime}$ as two duplicate organs, either of which might be educated to perform all the cerebral offices, we have to consider it as a double organ whose functions are partly separate and partly conjoint. That is, the facts point to the conclusion that (a) there is a class of functions over which the hemispheres have conjoint dominion: functions which they may perform together and which either may perform alone, and functions which they must perform together and cannot perform alone; and (b) there are functions which are peculiar to each alone: which one must perform alone, and in which the other never has a share.

The great divisions of function may be stated in general terms under three heads in accordance with the facts now presented.

1. Purely reflex functions are presided over by the spinal cord and lower centres.

2. The automatic functions proceed out from the " central" and "tegmental" systems of centres.

3. Sensation and voluntary movement have their seat in man in the cortex of the brain. Secondary-automatic reactions are those which have passed down from the hemispheres into the lower systems, in such a way that while the connections remain with the hemispheres, yet the latter are no longer necessary to bring these reactions into play.

\footnotetext{
' See his popular statement in the Forum, vol. v. No. 2, p. 169.
} 
If 1 . and 2. be considered together as giving only one degree of complexity, and 3 . be added as giving another degree, we may show their relation by Fig. 10.

For convenience in later discussion, the higher reaction may be taken alone and simplified, as in Fig. 11, called the " motor square;" in which we have the three elements as before $(s p, m p, m t)$ with an added element $(m c)$, i.e., the consciousness of movement accomplished (represented by the dotted line [mc] in Fig. 10).

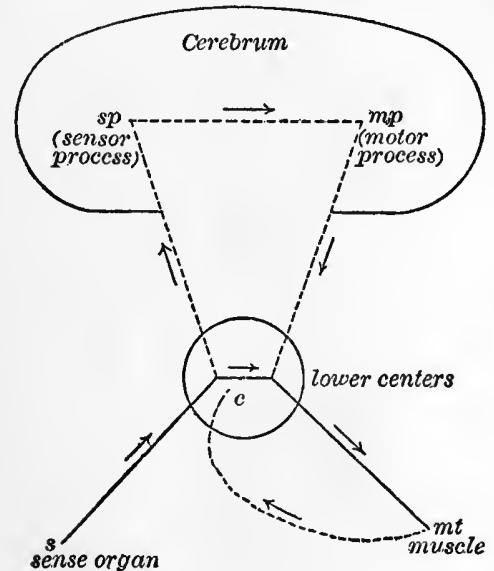

FIG. 10.

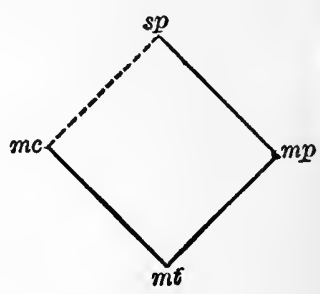

Fra. 11.

$s, c, m t=$ reflex circuit (1. and 2 , of text).

$s, c, s p, m p, c, m t=$ voluntary circuit (3. of text).

The degree to which the cortex serves the purposes of mind above the bare reception of present stimuli and mechanical reaction upon them, is seen in the behavior of animals deprived of the cortex. ${ }^{1}$ Frogs and pigeons have been fully tested in view of this question. It is found, in brief, that the life and reactions of the creature are unimpaired as far as the immediate environment is concerned : it lives, breathes, flies, sees, eats, carries out

${ }^{2}$ See James, loc. cit., I. pp. 14-27 and pp. 72-80, with his references to Goltz and Schrader (Pflüger's Archiv, vols. 41, 42, and 44). 
all reactions of response to direct stimulation. But it fails to respond to remote stimuli : the reactions are for the most part uninfluenced either by the past or the future. The creature lacks spontaneity. Memory has disappeared; so have generalization and purpose. The creature has sensations, but not perceptions, as far as a line can be drawn between these states. It fails utterly to recognize, and it fails to attend. It is plain, then, that such a hemisphereless creature lacks largely the coördinating, retaining, relating, in a word the apperceiving, function. It illustrates perfectly what, on Hume's theory of knowledge, ought to be the condition of us all. The terms psychic-blindness, psychic-deafness, etc., are given to this condition, in which there is no physical blindness, etc., but in which sensations have lost all mental meaning.

As for particular reactions, however, the greatest difference is found in different animals. In dogs and birds many functions are performed by the lower centres which are presided over by the hemispheres exclusively in monkeys and in man. This illustrates what has been observed above, i.e., that what is at one time reasonable and intelligent may become nervous and mechanical: and this consideration, based upon extended experimental proof, leads us to recognize below the great elasticity of the system as regards specialization. When these maimed animals are kept alive, their condition improves, and they begin to get something of their intelligence back again.

In man the destruction of the frontal lobes seems to bring about a higher kind of "psychic blindness :" a loss of voluntary attention, coördination, and thought. The hypothesis is widely current that these lobes are the final centre of convergence for the connections between the sensory and motor centres of the brain.' The loss of connection between this seat and any other area cuts the latter, with its store of memories, off from its full rôle in the mental life. For example, speech may be impaired

'A late advocate is Biunchi. See Amer. Journ. Psych., IIr. p. 551. 
by the loss of any one of three functions located in different areas, i.e., word-seeing, word-hearing, and worduttering. ${ }^{1}$

This evident division of work throughout the parts of the system led the older physiologists to propound the theory of "specific energies." E. H. Weber gave currency to the doctrine that each separate sense-nerve reacted by reason of a separate specific kind of force. The optic nerve, for exampie, has a specific optic-nerve force, the auditory nerve an auditory-nerve force, etc. This multiplication of forces was made to extend to different areas wherever their functions wereclearly distinguished. The next paragraph will show that this theory is now abandoned. The fact of necessary conjoint action of the hemispheres also leads to the limitation of the principle of specialization which follows. The dynamic conception of the system would lead us to expect it.

II. Principle of Indifference of Function. The principle of indifference includes the class of facts which show that the nerve-courses are not the agents of different or specific forces, but parts of a common system and agents. of a common life. As a matter of fact, we find that different courses can be made to perform each other's function. If a piece of sensor nerve be joined to a cut end of a motor nerve and grow in place, it will conduct the motor impulse continuously with the motor piece. ${ }^{2}$ The contrary is also true. The range of such experiments is very limited, since it is impossible to exchange the end-connections of nerves either centrally or peripherally: but the facts at hand establish conclusively the principle of indifference as regards the sensor and motor nerve-tracts. In its appliction to the centres the same principle has a different name, since it takes a somewhat

${ }^{1}$ See Wundt's diagram of the "apperceptive centre," Phys. Psych., 3d ed., r. p. 236.

'See Bert's successful experiments at reversing a rat's tail; also. Kühne, Archiv für Anatomie u. Physiologie, 1859, and Proc. Roy. Soc., xurv. p. $435 \mathrm{f}$. 
different form of manifestation, i.e., the principle of $s u b$ stitution.

III. Principle of Substitution. The question here is this: Can the nerve-centres be made to take up each other's function? Researches in cerebral localization, chiefly upon animals, tend to show that such a substitution of function is possible, at least to a limited degree. The removal of a cortical centre, which occasions loss of one of the special senses, say sight, or the loss of control over a certain muscular area, seems to be made good by the assumption of the deranged function by a contiguous or, at least, a connected centre. At any rate, the animal recovers if kept alive a sufficiently long period. The word "seems" is used advisedly, for it is still uncertain whether the loss of such a function is due to the destruction of the entire apparatus normally reacting to this function, or to its partial loss, the remaining elements being temporarily inhibited by so-called "physiological shock," " or, in the case of electrical stimulation, by diffusion of the current. The latter is known to be the case in many of the experiments on brain-tissue, especially when the surgical method is employed without the extremest care. This latter view is also supported by the remarkable fact that in the monkey and man these substitutions are exceedingly rare; a result we would expect on the shock theory, considering the higher degree of delicacy and differentiation attained by the system in these higher organisms. Yet in the case of rabbits and dogs, such substitution of function, notably of the sightfunction, is probably established on a firm basis.

The physiological ground of central substitution is therefore a legitimate problem in any conception of the nervous system as a whole. The conception here advo-

1 Cf. Lewes, Phys. Basis of Mind, p. 182. 
cated meets this demand with sufficient clearness. In organisms in which the reflex reactions predominate, in which the "downward" growth has led to the consolidation of the greater part of the system in ganglionic centres, we would expect that the higher functions, the centres for complex delicate movements, would be more dependent and unformed. Consequently, when they are interfered with, the ganglionic centres, being still in close anatomical connection with them, would regain the function which they formerly performed. Thus sensorimotor ganglionic connections which have fallen into disuse through the growth of higher centres recover their lost activity under the stimulus of a serious and dangerous lesion. It is nothing more than a reversion of function by a reverse process of adaptation.

On the other hand, in the case of man, the law of "upward" growth has reached its fullest application: the cortical centres have become independent of their ganglionic confrères, and, in their loss, an irreparable damage is sustained. In this latter case, it is a general in the army who has fallen, and no subordinate officer can fill his place: in the former case, it is a captain that is lost and his lieutenant is easily promoted. ${ }^{1}$ In the lower animals as well as in man, the automatic functions are relatively independent of the higher centres.

Both the foregoing principles, Indifference and Substitution, become clearer when we revert to the dynamic aspect of the system. If it be true that the whole system is one charged with a common force, and reacting in support of a common life, then we would expect that the courses would be interchangeable. They would be principally avenues of transfer of a tension over and above their separate dynamic capacity; and why should they not serve for such transfer in one direction-or for one sense-as well as another? And as for the centres, their substitution would be limited only by the conditions of their anatomical connections: the very distinction

1 This hypothesis is recognized by Foster, loc. cit., 5th ed., p. 1062. 
we were led to make above, in accounting for the differences between men and dogs in this respect. ${ }^{1}$

IV. Principle of Speciflc Connection. The limits which the growth of the organism sets to the substitution of functions find their expression in what is called "specific connection" through the system. By this principle is meant, in general, two things : first, that nerve-courses are specific only according as they have certain welldefined connections at centre or periphery. These connections keep the courses to an invariable function. The optic nerve has a specific connection with the retina and with the optic centre in the brain; the auditory nerve with the ear and the centre for hearing; and so on. In this case, it is the end-organ or the centre which is specific, not the nerve-tract. And second, it means that nerve-centres are specific according as their connections necessitate their reacting to a specific stimulus. The optic centre has specific connections with the retina through the optic nerve; the centre for sounds with the ear, through the optic nerve, and so on. Now there are as many of these specific connections as there are kinds of stimuli issuing in motor reactions. Consequently, the only specific things after all are the stimulus and the movement.

This, again, is a conclusion quite in harmony with our dynamic conception. There is nothing specific about a sewing-machine except the force that runs it and the garment it sews : its function is sewing, not revolving, nor pricking, nor buzzing, however specific each one of these, considered for itself, might be, apart from the sewing function.

V. Principle of Summation of Stimuli.' If the stimulus more than overcomes the arrest in a given case, there

1 For an able recent discussion of the facts involved, see James, loc. cit., I. pp. 67-72.

For references on physiological summation, see Jumes, Princ. of Psych., I. pp. 82-83. 
is left over, so to speak, a surplus of positive energy, or positive " molecular work." This positive molecular work is work of reaction, or exhaustion of the system; negative work being inhibitory or conserving. This surplus represents, therefore, a disposition favorable to a second stimulus of the same kind. We have, therefore, here a certain summation of stimuli in cases of recurring excitations of the same character. After moving the thumbs in a certain rotatory manner a certain number of times, we say they are "ready" for that movement; they have taken on a disposition to react to the same stimulus again. This union of former stimuli with later in the nervous centre, ${ }^{1}$ giving an easier and smoother reaction, is the phenomenon of summation. Its most remarkable exhibition is seen in cases in which the earlier stimulus is not sufficient to overcome the arrest or inertia of the centre, and does not give a reaction at all; so a weak electrical stimulus : even here we find the centre so "prepared" by this insufficient stimulus that it responds when that identical stimulus is repeated a sufficient number of times. The most favorable interval between such shocks is about .001 second. The successive blows of Savart's wheel upon a metallic tongue give an audible sound when a single such blow is inaudible. The application of this principle to disprove unconscious sensations has already been made.

The different senses vary very much in the interval of time necessary between successive stimulations to prevent summation or fusion; the finger discriminates 1000 touches per second: an interval of .002 sec. is sufficient to keep sharp sounds apart: electric shocks fuse if more than 60 occur per second (forehead; but in

${ }^{1}$ Not in the courses : cf. Richet, Recherches sur la Sensibilité, pp. $174 \mathrm{ff}$. See also his figures showing the phenomena of summation as graphically recorded (pp. 171, 176, and 186).

${ }^{2}$ See Senses and Intellect, chap. Iv. § 2 . 
some parts of the body the number is much less). With sensations of sight, the fusion occurs across a greater interval, say .05 sec., by reason of the persistence of optical after-images. ${ }^{2}$

\section{§5. Final Statement of Nervous Function.}

We are now in a position to give the general conception of nervous function in broadest statement: a statement the accepted terms of which have great psychological significance. All the phenomena of consolidation (schöpfen, "downward growth"), on the one hand, illustrate what is known as the law of Habit; all the phenomena of specialization (" upward growth ") illustrate the law of Accommodation; and the results of the two together, as transmitted by generation, illustrate the law of Inheritance.

I. Law of Habit. Physiologically, habit means readiness for function, produced by previous exercise of the function. Anatomically, it means the arrangement of elements more suitably for a function, in consequence of former modifications of arrangement through that function. Psychologically, it means loss of oversight, diffusion of attention, subsiding consciousness.

II. Law of Accommodation. Physiologically and anatomically, accommodation means the breaking up of a habit, the widening of the organic for the reception or accommodation of a new condition. Psychologically, it means reviving consciousness, concentration of attention, voluntary control-the mental state which has its most general expression in what we know as Interest." In Habit and Interest we find the psychological poles corresponding to the lowest and the highest in the activities of the nervous system.

'Fick, Hermann's Handbuch, III. 1.

'Sce the discussion of Interest below, Ch»p. VII. $\S 1$. 
III. Law of Inheritance. The broader statement of nervous development takes the subject out of the experience of the individual into that of the race or species. Not only is the nervous system an integration during the life of the individual organism, but it proceeds upon the integrations effected in the life of earlier individuals of its kind. This means that potential integrations and systems of integrations are inherited. The offspring of a rabbit is born with the nervous system of a rabbit: that of a man with the nervous system of a man. There is, therefore, continuity of development throughout the life of a species, its limits being the limits of possible generation.

This law widens our outlook and introduces us to the philosophical doctrine of evolution. As a philosophical doctrine, we have nothing to say about it here. We have indicated above the extreme limit to which general psychology may go in the matter of the nervous basis of consciousness ; namely, inheritance within the limits of possible generation. Even though, as a philosophical doctrine, nervous evolution be considered established, psychology would still await the establishing of a corresponding doctrine of mental evolution, before the discussion would have special interest and value for her interpretations. ${ }^{1}$

On the nervous system, consult: the physiologies generally, of which Foster, T'extbook of Physiology, 5th ed., part III, is very reliable; on special points of research, Hermann, Handbuch der Physiologie; on brain anatomy and physiology, Edinger, Structure of the Central Nervous System; Obersteiner, Central Nervous Organs ; Ferrier, Functions of the Brain, 2d ed.; Wundt, Physiologische Psychologie, 3d ed., I, erster Absch.; Ladd, Elements of Physiological Psychology, part I, also Outlines of Phys. Psych.; James, Psychology, I, chaps. II. and III. Special articles of great value are found in Archiv für Anatomie und Physiologie (Du Bois-Reymond's) and Archiv für die gesammte Physiologie (Pflüger's).

Further Problems for Study :

Physiological methods and results.

Histology of the nervous system.

Physiology of inhibition.

Heredity.

Organic evolution. $\S 7$.

${ }^{1}$ See further remarks on psychological inheritance, below, Chap. III. 


\section{CHAPTER II.}

\section{THE NERVOUS SYSTEM AND CONSCIOUSNESS.}

THE most general problem involved in a conception of the relation of body and mind is the rise of consciousness. Yet the problem has psychological signifieance mainly as throwing possible light upon the nature of consciousness. With the philosophical question as to whether the nervous system produces consciousness, or whether consciousness is something essentially immaterial, however it may be entangled in material conditions, we are not here concerned. Indeed, it may be said further that empirical psychology has no right to ask such a question. We found the fundamental postulate of such a science to be consciousness, inner aspect; and to go back of this postulate is to subvert the science altogether. Granted a consciousness, however it arose, our questions are: What is in it? and, What is it capable of? The reader is, therefore, here concerned with the class of problems which arise from the concomitance of certain physical changes with certain aspects of consciousness.

\section{§ 1. Nervous Conditions of Consciousness.}

General Conditions. This general question is in too unsettled a condition to detain us long. There are two great theories of the physical basis of consciousness : the first, represented by Mr. Lewes, ${ }^{1}$ holds that the

${ }^{1}$ Held also by Bain (Emotions and Will, Appendix A), and in a modified form by Wundt. 
nerve-process, considered in its most general form as irritability, is everywhere conscious. On this view, consciousness is distinguished in a twofold way from the individual circumscribed area of personal experience. In the first place, it is distinguished from selfconsciousness ; and, in the second place, from the vague early consciousness of the child, which exists only in connection with the brain. Each nervous centre, each so-called arc, has its own consciousness, and the ordinary consciousness of the individual is only the highest of many that we all possess. The brain-consciousness is the only one we are conscious of, so to speak; but there is consciousness in the spinal cord and in ganglia wherever we find them. The other theory, or class of theories, holds ${ }^{1}$ that a given degree of development is necessary before consciousness is found at all. In the development of the system, therefore, consciousness appears only at a certain stage of integration or "upward growth." This theory is generally accepted, though for purposes of division rather than from positive argument. In the nature of the case, it is impossible to disprove consciousness in lower centres, as has already been shown in arguing against the unconscious. ${ }^{2}$ The distinction, however, is held to be necessary, as indicating the bounds of the mental objectively considered. For instance, Romanes ${ }^{8}$ and James give selective choice or adaptation as the criterion of mind or consciousness; but the difficulty of saying whether the adaptations of the brainless frog, for example, show conscious choice, or mere delicacy of mechanical reaction, remains just as great.

${ }^{1}$ So, among recent writers, Maudsley, Schneider, James, Ferrier and the psychologists quite generally.

'Senses and Intellect, chap. Iv.

3 Mental Evolution in Animals, p. 49.

4 Schneider attempts to mediate between these two views, as I understand him, Thierische Wille, pp. 151-4; he holds that all reflexes: 
It also seems true that consciousness represents a condition of slow, difficult, and impeded-consequently of highly developed and well-balanced-integration. The smoothest reflexes are not conscious; the hardfought decisions are most conscious. It seems likely, therefore, that some degree of inhibition is necessary in the nervous basis-at any rate for vivid consciousness.'

On the other hand, there are considerations which are giving more prominence to the view of Mr. Lewes at present. They tend to show that our distinctions are arbitrary, and open the door at least for presumptive evidence that consciousness is coextensive with nervous reactions. Among these considerations are recent experimental proofs of multiple personalities which may be induced in the same nervous organism in the hypnotic state. ${ }^{2}$ The explanation is at least a tempting one, that, the higher centres being inhibited, their conscious content is wanting, and the lower centres supply experience which was before outside the conscious area. Again, in the scale of animal organisms. it is diffcult to draw a line denoting the point of nervous complexity below which there is no consciousness. The fact of a possible substitution of function between the brain and spinal ganglia spoken of above, would indicate a possible common element of consciousness.

To these considerations we will not add the metaphysical one that perhaps the integration of consciousnesses in an allembracing divine consciousness, so long sought by thinkers who desiderate a monism of mind which is still not pantheism, may find a valid analogy in the integration of subordi-

involve consciousness at their first establishment in race experience, but have become independent of it, just as a chain of voluntary movemeuts comes to need conscious volition only at the beginning of its play.

'See Mr. Spencer's hypothesis to explain this nervous condition, Princ. of Prychology, $\$ 196$.

'Pierre Janet, Automatisme Prychologique. 
nate consciousnesses in the unit personality of man. At any rate, it is a ready hypothesis, and in the present state of discussion Lewes has the apparent advantage, however much he would deprecate this metaphysical application of it. The theory that consciousness occurs wherever we find nervous elements has a broad philosophical construction in the socalled "double-aspect theory," according to which mind and matter are a single fact, looked at from two aspects, the inner and the outer. If consciousness is present in the elements of the nervous tissue, apart from the unit consciousness of the organism as a whole, what limit can we set theoretically to the recognition of an inner aspect in objects in nature where a unit consciousness is entirely absent? Beginning with Leibnitz, this theory finds its latest, and perhaps its acutest, advocate in Prof. Wundt. ${ }^{1}$

Particular Conditions. A further question arises as to the immediate conditions of consciousness in the nervous centres. Given a nervous organism capable of consciousness, on what particular state or aspect of it does the continuous presence of consciousness depend? Here, again, recent views are little more than guesses. The view supported by Herzen ${ }^{2}$ seems to have most evidence, i.e., that consciousness arises from the breaking down or expenditure of the cellular structure in the highest centres. This is concluded from the fact that the attention, a state of concentration and expenditure, is the state of most vivid consciousness; that consciousness is most vague and indistinct when no brainwork is being done, as in cases of dolce far niente or diffused attention; that unconsciousness is most nearly reached in sleep and analogous states when all brainprocesses have subsided from the lack of sensory stimuli or motor impulse. The chemical results of active thought, increased heat, and organic-waste deposits in the brain, would indicate chemical work and disintegra-

1 For a criticism of Wundt's view see the writer's article on " $R e$ cent Materialism" in the Presb. and Reformed Review, July 1890, p. 364.

' La Condizione fisica della Coscienza, reprint from the Proceedings of the Academy of Florence, 1879. 
tion. Such a theory, however, to be consistent, would have its application only after the organism had attained the requisite development in general structure for the presence of consciousness-if we hold the current theory that consciousness is not coördinate with nervecentres. Perhaps the possibility of applying the present hypothesis of Herzen to nerve-tissue generally may be an additional point of proof of a coördination of consciousness and nervous function throughout.

It is also true that consciousness depends upon the normal condition of the mechanism as a whole. Any failure in the blood-supply (anæmia) leads to faintness and fainting, and the same result often follows from congestion of blood in the brain (hyperæmia). In general we may say that the healthful activity of the brain, in its normal physiological relations, gives clear consciousness. It should be borne in mind, also, that all hypotheses as to the conditions in which it arises, shed no light on what conscionsness is. On this point even the biologist Schneider is clear. ${ }^{1}$

\section{§ 2. Sentience and Sensibility.}

It has become apparent that nervous activity considered for itself alone does not bring us into the range of psychological science. However we may decide the inquiry as to whether such activity is ever entirely free from consciousness, it is yet true that it may be quite outside of what is called the individual's consciousness. The man is not conscious after the guillotine has done its work, however active the nervous reflexes of his limbs may be, and however firmly we may believe that his spinal ganglia have an inner aspect. In other words, the greater part of our ordinary nervous reactions are not above the threshold of our conscious lives. So we reach a distinction between sentience as a nervous property and sentience as a conscious phenomenon,

Thierische Wille, p. 127. 
between sentience and sensibility. Sensibility is synonymous with the usual consciousness of the individual's experience, and sentience is the nervous function which may or may not be accompanied by consciousness or inner aspect in general.

The criterion of sentience is the general criterion of the nervous, i.e., reaction. Wherever a stimulus is responded to, there is sentience, from the sensitive plant to the palm of the hand. This is an objective criterion solely. The criteria of sensibility, on the other hand, are both subjective and objective. Sensibility becomes objective when it shows reaction plus selective adaptation: subject to the uncertainty already signalized as attaching to such a test, yet in the case of the human consciousness sufficiently clear for psychological purposes. The spinal reflexes in man, for example, which are mechanical after he is beheaded, are more or less under regulation and control in normal circumstances and so belong clearly to sensibility. And there is the additional subjective criterion of consciousness itself, the major or personal consciousness. Subjectively this test is decisive, but limited to our observation of ourselves. But it becomes of value also objectively ${ }^{2}$ when I learn that the major consciousness resides in the brain, and the destruction or incapacity of that organ destroys it. Whenever the brain is gone, that is, I know that the trunk before me has no sensibility, just as well as I know that I have no memories of a period of fainting. The former is known ejectively, the latter subjectively: from the fact that $\mathrm{my}$ friend gives no sign of pain when he sustains an operation under the influence of chloroform, I say I know objectively that he had no sensibility.

${ }^{1}$ Lewes uses the two terms in senses precisely the reverse of this, Phys. Basis of Mind, p. 222 ; i.e., to him sensibility is the nervous property everywhere ; so also Sergi, Psychologie Physiologique, p. 12.

${ }^{2}$ More properly ejectively in Clifford's phrase : the eject is some one's else mind as $I$ look at it. 
For a working test, therefore, of the limits of sensibility we may say that there is no sensibility (1) where there is no brain; (2) where there is no trace left in memory; (3) where there is no expressive or adaptive motor reaction. Yet in all of these cases sentience may be present, as the sensitive plant seems clearly to show.

The same distinction will be observed in the use of the two adjectives sentient and sensible. A reaction is sentient when it sustains the test of sentience, and sensible when it exhibits sensibility. Sensible is used exclusively, no room being found for the adjective sensitive, since its customary use vibrates between the two meanings given to sentient and sensible. We speak of a sensitire balance, or a sensitive plant, or a sensitive conscience. It is better to discard the word altogether, for exactness of meaning.

The meaning here given to sensibility makes it almost synonymous with feeling. ${ }^{1}$ Feeling is used in the discussion of the intellect to indicate either any particular affective state whatever, or the general condition of the affective consciousness. Sensibility is this latter : the capacity for feeling; the particular states of sensibility being feelings, sensations, emotions," etc.

Sentience and the Subconscious. The transition from simple sentience to the full consciousness is through a stage of subconscious modification. Most of the phenomena usually claimed as evidence of unconscious mind have already been seen to find their explanation here. ${ }^{3}$ On the side of the nervous system they indicate a stimulus and reaction too faint to reach into the sensibility. Yet they influence the conscious life and give it direction and intensity : a fact seen again on the physical side under the principle of summation of stimuli. Under this sensational or purely affective aspect, such

'So Dumont, loc. cit., p. 23 : as this writer suggests, the sclence of the sensibility in general should be called asthetics (pp. 23-25).

See general classification below, Chap. III.

' Senses and Intellect, chap. Iv. 
facts have been given sufficient recognition; there is, however, another aspect of consciousness, the motor aspect, in which their importance remains to be pointed out later.

\section{§3. Kinds of Conscrousness as Dependent on Nervous Complexity.}

1. Passive Consciousness. Such subconscious sensibility tends to secure recognition in the mental life as what is called passive consciousness, to distinguish it from the active forms which involve more or less attention. In most cases passive consciousness is, by its very nature, undetected, and it exists as a normal state apart from active consciousness only in lower forms of organic life or in very young children. In adult life we catch it most nearly when just beginning to recover from a swoon ; the sounds around us are heard, but have no meaning, relation, or escort. Of this state abstracted from the condition of our usual self-consciousness, we may make the following remarks: 1 . It is a state of pure sensibility or simple awareness. 2. It carries no reference to an external object or to the body, that is, no such reference inside the inner aspect: $:^{2}$ that the nervous process has a direct reference to a particular local stimulus is seen in the particular character of the reaction which follows in each case. 3. It has no reference to self as an object of inner apprehension, no voluntary effort known as "my effort." 4. It has no relational or apperceptive quality. It is not knowledge, but pure feeling. It is the hypothetical affective state in all its purity.

We say hypothetical, since philosophers will have it so : and certainly it is hypothetical to us as long as we are phi-

${ }^{1}$ See cases gathered by James, loc. cit., I. pp. 273-4.

2 "Placeless, objectless sensation"-Dömrich, quoted by Nahlowski. 
losophizing about it. But everybody is coming to accept it as the earliest form of consciousness. Even Prof.' 'T. H. Green," the arch-enemy of anything not " intellectually constituted," finds that his net has caught this fish when dragged for other gamo. 'To the evolutionist eertainly it is a necessary item; but if it is a fact it ought to be equally necessary and important to us all. Prof. John Watson, therefore, in replying to $\mathrm{Mr}$. Spencer on space-perception, " would be said by perhaps nine tenths of living competent psyehologists to be wide of the mark in bringing the following damaging (!) charge against Mr. Spencer, i.e., that he makes "the oecurrence of a sensation the same thing as the consciousness of that occurrence." When "love burns or remorse gnaws," the feeling as an oceurrence is exactly the same thing as my consciousness (not my knowledge) of it.

The philosophical bearings of this position may not detain us; yet, fortunately, the refutation of the materialistic position does not require any denial of facts. Granted a purely affective consciousness, entirely without relations of time, space, intensity, etc. ; how does the relational conscionsness arise? This is Prof. Watson's essential point, as our own, and the empiricists have never answered it.

The affective quality of sensations comes out most strongly in cases of massive or voluminous stimulation: here relations are at a minimum and sensibility is at a maximum. When one plunges into a very hot bath, the feeling experienced is so overwhelming that the knowledge that it is a hot bath, and that it is I myself who am taking the bath, occupies a very slight degree of consciousness. We can imagine the diffused wave of feeling that sweeps over the jelly-fish when an unwary insect settles on its exposed surface. In a case of severe toothache, also, what we really have predominating in consciousness is not knowledge, but feeling. As an immediate state of consciousness, we do not know that we have a toothache, we feel it."

The possibility of turning attention to a dim presentation and making it vivid shows that the cerebral basis of these lower forms of human consciousness is not one of separateness from the highest centres, but of com-

1 Prolegomena to Ethics, pp. 48-53, also 89, 124, and 125.

Mind, vol. xv. pp. 541-2.

${ }^{3}$ Senses and Intellect, treatment of Space and Time.

- Compare Bain's remarks, Emotions and Will, Appendix C. 
munity with them: indeed a nervous discharge already in voluntary operation may be diverted into a subconscious reaction without the attention." Passive consciousness, then, physiologically considered, is a state of temporary loss of tension in a brain-area which shares in the highest integration and instability.

2. Reactive Consciousness. By reactive consciousness is meant the state formerly designated as involuntary attention. In passive consciousness only the reception of stimuli is a matter of sensibility: here consciousness seems to attach to both members of the nerrous arc. There is as truly a reaction in consciousness as there is in the nervous system. We may accordingly analyze this form of consciousness for purposes of treatment into three elements, corresponding to the three elements of the nervous arc. First, the receiving consciousness, the stimulus-say a loud unexpected sound; second, the attention involuntarily drawn, the registering element, as appears below; and third, the muscular reaction following upon the sound-say flight from fancied danger. The analogy accordingly between the typical brain-process and the typical mental process fiuds here its most general force and demands the most careful treatment. Questions of the most radical philosophical importance

${ }^{1}$ In my writing I have discovered a happy case of this. While discussing the nature of the nervous process which underlies the so-called muscular sense, the words afferent and efferent were of frequent occurrence, and lay throughout in subconsciousness. After writing a sentence containing the word superficial I found that I had written instead superferent. In such cases a very unstable nervous connection is brought up to the status of a real subconscious reaction by some one element (here the letter $f$ ) common to it and another similar reaction voluntarily excited, and the latter nervous discharge is diverted, without drawing the attention, into the muscular channel of the former. The ordinary substitution of single letters, etc., in writing, are simple variations in a common motor discharge, and do not presuppose even a subconscious process. 
begin here. They are necessarily postponed until we complete our survey of this general analogy.

The exigencies of clear treatment compel a division of material which logically falls together under any adequate view of our present topic. The nature of the stimulus in reaetive consciousness must be thrown into a separate chapter; that is, it falls under feeling. The nature of the consciousness of the muscular process, on the contrary, is so bound np with the phenomena of will that it is better taken up in that connection. The nature of the central process, also, attention itself, falls largely under the intellectual function, and has had notice there: as a matter of feeling it is discussed later. ${ }^{2}$ It may suffice, therefore, to have suggested these themes at this point.

Characteristics of the Reactive Consciousness. In general, this form of consciousness is distinguished by a feeling of expenditure. Attention always means expenditure, even when quite involuntary. Any further designation would only becloud a sensation which every one can point out clearly enough in his own experience. As a feeling, it seems to belong between the consciousness of the stimulus and the consciousness of the muscular reaction.'

Again, the reactive consciousness has an additional element which we call the sensation of fatigue. This sensation is distinct from that of expenditure, and arises only after prolonged attention or in conditions of antecedent nervous exhaustion. As to what this feeling is, again, no further description is necessary now.

Moreover, on the muscular side we find two different classes of effects: the reactive effects peculiar to the particular stimulus, and besides these the peculiar muscular accompaninents of attention itself. The latter are constant, and the former vary with the stimulus. For example, a student hears his name called suddenly and loudly. The particular reaction habitual to such a 
stimulus is a speech-reaction-the response, Hullo! or Yes! But he turns his attention to the source of the sound, and by so doing brings into play a different set of nerves and muscles. Now of these two reactions it is the speech-reaction which answers in consciousness to the motor side of the nervous arc stimulated by the sound, and it is only this that we can say follows the attention without finding ourselves on debatable ground. The attentive movements seem to belong peculiarly to the attention itself, and so fall under the central element. in the typical reaction.

Consequently, in the motor phenomena of attention there are two very distinct elements which subsequent.

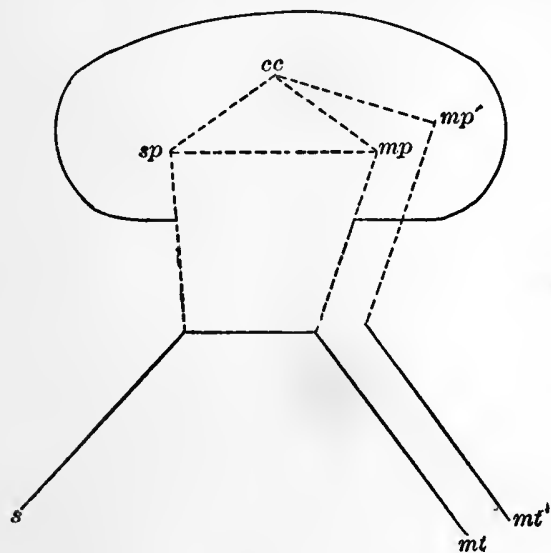

Fra. 12.

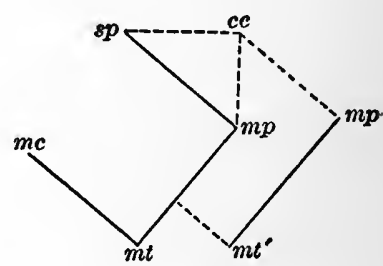

Fig. 13.

$s p, m p, m t=$ motor reaction.

$s p, c c, m p^{\prime}, m t^{\prime}=$ motor accompaniments of attention.

discussion must not confuse: the motor effects of the stimulus which is attended to, and the motor accompaniments of the attention itself. This may be seen in Fig. 12 , in which a new element ( $c=$ coördinating centre) is added as the central process of attention. A new motor process $\left(m p^{\prime}\right)$ is stimulated, and this produces new muscular movements $\left(m t^{\prime}\right)$. The ordinary reaction also. 
takes place ( $m p, m t$; in this case speech) following from the ordinary stimulus ( $s p$; in this case, sound). The matter is again simplified in the "motor square" diagram, Fig. 13, which, with Fig. 12, should be compared with Figs. 10 and 11, p. 42.

The physiological process of expenditure seems on the surface to be analogous to the mental process; ${ }^{2}$ that is, if physical expenditure be the basis of sensible expenditure, our analogy is satisfactory. This is found in the positive discharge of a centre on an efferent course: and the analogy would seem to require a form of consciousness arising from the motor discharge itself. The feeling of fatigue, also, seems to have a sufficient physical basis in the lower potential of the motor elements in consequence of frequent discharge. They have become drained and to a degree incapablo of farther reaction.

Divisions of the Reactive Consciousness. Apart from the passive consciousness already mentioned, which forms, theoretically, the purely affective basis of a reaction, a further distinction may be made according as we view the reaction simply as motor sensibility or as the medium of the relational process of attention. The attention always establishes relations. This relational process is the foundation of knowledge and gives us, whenever it occurs, the apperceptive consciousness. The muscular sensibility, on the contrary, meaning all sensations involved in muscular movement, whatever their seat and physiological basis, may be called the motor consciousness." The apperceptive consciousness was the subject of the former volume.

III. Voluntary Consciousness. Voluntary consciousness may be characterized by several new affective elements-new modifications of sensibility. Without anticipating later analysis, we may say that it exhibits, first, deliberation. By this is meant, in general, a doubleness 
of sensibility, a consciousness of being drawn apart, or of inward conflict-to limit the case to the feeling aspect, apart from the play of ideas involved. This feeling of deliberation leads on to another element of sensibility, namely, the feeling of decision or consent ; in which the doubleness spoken of is resolved in a pleasant unity of consciousness again. And further, we find another possible element, apparently distinct from the preceding, the feeling of effort. In this sensation there is an active identification of ourselves with the reaction decided upon; a conscious putting forth of ourselves to reinforce our decision. Any analysis of volition must, at least, take account of these three distinguishable aspects of sensibility. ${ }^{\prime}$

Now it is in the selective and inhibitive functions of the nervous system that the physical basis of the voluntary consciousness is to be found. As far as such selection and inhibition are conscious at all, they have probably the essentials of conscious volition. Of the three sensible elements involved, the first and second have clear physiological analogies. Deliberation in consciousness is analogous to dynamic complexity and instability in the brain-centres: there is such a thing as temporary balance in the nervous system, and it suggests itself at once as the physical counterpart of mental hesitation. Inhibition also, as far as our physiological knowledge goes, seems to have full conscious value here. Decision, as following upon deliberation, is again analogous to the state of central readiness for the discharge of nervous force, when the equilibrium is destroyed and the motor outburst only waits for the requisite stimulus, to take its outward course. Decision proper therefore precedes the sensation of expenditure, the latter being full consciousness of a motor discharge.

${ }^{1}$ For detailed consideration of these three features of voluntary consciousness, see below, Chaps. XV and XVI. 
With effort, however, the case is on the surface different. There is no evident nervous function corresponding to this state of sensibility; that is, no function not already supplied with its conscious analogy. The question of such an analogy or physical basis of effort, therefore, comes finally to wait upon a more thoroughgoing mental analysis of this sensation. If effort be reduced to expenditure and expenditure to incoming sensations from the muscles, then there is no need for such an analogy; but if effort resist further analysis, either physiology is as yet at fault, or there is no possible physiological construction of effort.

It is important that the present state of this question should be clearly understood. The mental conditions of volition appear in the proper place below. It is quite possible that future physiological research may find an additional element of nervous integration underlying the selective function, especially as our hypotheses as regards the nature of inhibition are as yet incomplete. Indeed it might be well to anticipate such an advance in our knowledge. But it has not yet been made, and hence the so-called reduction of volition to a consciousness of a physiological complex, in the absence eren of requisite nervous analogies, are crude and unreasonable. They proceed upon an inadequate analysis and statement of the conscious facts. ${ }^{1}$

In addition to these elements of sensibility, the voluntary consciousuess shows the elements already found to characterize the reactive consciousness: they need not be again enumerated. Whatever is true of attention at all is true also of voluntary attention. The apperceptive form of reactive consciousness becomes, however, more conspicuous in the voluntary.

The results of the exposition of this section may be set forth in a table showing the various aspects of consciousness, as the consideration of the nervous system brings them to view:

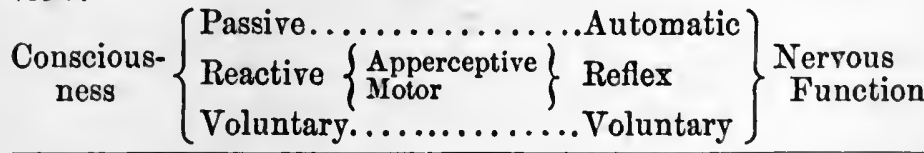

1 Notably Maudsley. Mr. Spencer shows discretion, if nothing inore, in letting this question alone. 


\section{§ 4. Analogies of Function.}

More general facts of consciousness come into our field of vision when we ask for the mental counterparts of the "Principles of Nervous Action." Such facts are largely interpretations belonging to an advanced stage of the introspective and descriptive science of psychology -just as the "principles" belong to the systematic results of anatomy and physiology. As the statement of the latter was found above to be somewhat hypothetical in many cases, so the statement of their correspondence with mental principles must be as yet quite fragmentary and incomplete.

Nervous Retention and Consciousness. The psychological conditions of retention have already been fully considered.' The physiological facts required to support the view already taken of mental retention so-called, are now seen to be real elements in a true conception of the nervous system. ${ }^{2}$ If the conditions of conscious memory are fully met by this explanation, and if physiology affords us the requisite data on the side of the nervous organism, then the case seems to be made out.

Briefly expressed, therefore, the mental principle of retention is the principle of mental growth; a growth which is at every stage dependent upon true physiological retention. My mind grows because it has in its remembered stores things new and old, and because its synthetic constructions are preserved as a basis for future apperceptive syntheses. But there is no preservation of single states-those subject to the integrating (associating) process-as acquisitions, apart from and out of this activity of synthesis.

I Senses and Intellect, chap. IX.

${ }^{2}$ Above, Chap. I. $\$ 2$. 
On the side of the affective consciousness, mental growth takes the form of increased susceptibility, on the one hand, to new stimulations, and diminished susceptibility, on the other hand, to habitual ones. On the relational consciousness the effects are quite the contrary. When a stimulus is old, we feel it less, but discriminate it more: when new, we feel it more, but discriminate it less. This is simply to say what we would expect, that the nervous process in becoming fixed favors the establishing of the fixed relationships of knowledge, but hinders the intensity of consciousness as sensibility-which latter has been seen to depend upon instability in the nerrous organization.

Occasion has already arisen, also, to say that the evidence both physical and psychological of retention is functional evidence. We know that we have certain memories only by remembering them--only when we pass out of any such thing as retention proper into the function of reproduction. So also any evidence in detail of physiological retention, or minute modification of structure, becomes evident only in the performance of the organic function of which it forms a part.

The history of this functional retention in a particular individual is an interesting page of physiological psychology. The growth of body and mind is so close and intimate, as regards retention, that our idea is clear only when it includes both. The first random movements of the child betray inherited tensions and ready-formed paths for nervous energy: and the first intelligent choices of the child show an even finer inherited mental organization. As the nerve-centres become more complex, their tensions toward repetition of discharge become stronger: and as the child grows to appreciate alternatives, it discovers their strength or weakness in lines indulged or neglected. Pathways of indescribable intricacy, an organic network of nervous connections, serve 
as a hint to the varying phases of impulse and motive that sway the preferences of the adult man, and picture the possibilities of the life of association and interest in which all his activities find their play.

It is not in place to dwell here upon the possibilities of memory that such a conception opens up. Whether it be true or not that no experience of our lives is ever lost as an individual memory, it is certainly true that it is never lost as an element of our present. Physiological integration is possible only by reason of minute increments in experience; and each such increment, if sufficient to work a change at all, takes its place as a stone in the wonderful structure which we call the nervous system. To question whether a mental fact may be remembered again is to question whether its effect in consciousness at first gave it the right to a place in the mental synthesis in which reproduction consists.

The analogy here between physical function considered as a reinstatement of a nervous state and mental function also considered as a reinstatement, is so close that it is often considered a conclusive proof of the identity of the two. Yet the irreducible character of the apperceptive process, as already insisted upon, meets us both in the first experience and in its reproduction. The dependence of mind upon physical retention is entirely consistent with its independence in presentation and representation, and the former fact satisfies the demands of the analogy as far as it is legitimate. The individual movements of my pen do not involve my attention sufficiently to become fixed as memories; and so it is impossible for me to remember all the movements of a hundred pages of manuscript. But every movement has been a nervous experience, has been integrated in the nervous system as a whole, and my handwriting in the future will be different for every letter I have written. 'This is simply to say that physical elements suffice for retention, but not for finished memory.

Principle of Specific Connection and Consciousness. Another general principle of the mental life comes to mind when we seek to interpret specific nervous connections, i.e., the principle of association. It is undoubtedly true that mental association is possible only when the specific brain-centres which the associated elements involve maintain organic connections with one another. 
This is so true that if certain brain-areas be removed, certain well-defined classes of memories disappear: and the other centres which remain do not perform their function together, if their connection depends upon the presence of the lost areas. The best example of this is found in the different forms of aphasia. The full "speechfaculty," so-called, depends upon the maintenance of the proper dynamic connections among several different brain-areas. A minor application of the same principle leads us to what we may call the "specialization of mental function"-carried to a mechanical extreme in the old "faculty-theory." :

Principle of Summation of Stimuli and Consciousness. The summation of stimuli not only avails to bring a reaction over the threshold of sensibility, but it applies within the sensible world in differences of sensational and emotional intensities. The general law of increasing intensity in relation to increased stimulation is already familiar. Weber's law is simply a principle of the summation of conscious intensities. That there is such a summation is seen in the plain fact that one light is brighter to us than two; that the loss of a hundred dollars gives us more anxiety than the loss of fifty; and that the effort of memorizing a dozen words is greater than that of memorizing six. This application of the principle, however, is confined to facts of positive sensibility. That we are not able to argue a conscious result from the summation of unconscious elements we have already had occasion to show. ${ }^{2}$

Inhibition and Consciousness. ${ }^{3}$ The clearest and most important kind of inhibition in consciousness is

1 Wundt warns us, however, against too close analogy between brain-connections and formal association of ideas.

"Senses and Intellect, chap. IV. \$2 and chap. vIr. \$4.

'See a clever article by Binet, L'inhibition dans les phénomènes do conscience, Revue Philosophique, Ang. 1890. 
voluntary. We find it possible to exert a direct force of will to prevent or cut off a nervous reaction or train of thoughts. This has already had mention above.

Other less evident applications of the principle may be made within the sphere of sensibility. As a principle of belief it has already been mentioned under the socalled Law of Contradictory Representation: ${ }^{1}$ its bearing in this connection, however, is not upon belief, but upon actual conflict and repression among images. The question is: Are there cases of antagonism among states of consciousness analogous to the positive and negative aspects of nervous tension and discharge?

Very little reflection is needed to show us that our conscious life is a complex of such conflicts, repressions, and reinforcements among images almost as elaborate as the system of tensions we have found in the nervous. system. This aspect of our mental life has been emphasized by the Herbartian psychologists, and their service, when freed from physical terminology and mathematical expression, is a real one. It is only necessary to compare our ordinary waking life, its reasonableness, continuity, and soberness, with some of our dream-states with their incoherence, extravagance, and feeble semblance: of reason, to see what we owe to inhibition considered as a balance of mental tensions. The representation of a winged horse or a scaly mermaid is just as possible when we are awake as when we are asleep: why isn't. it just as likely to arise in consciousness and remain? Simply because other images of horses and maids inhibit. the wings and the scales with all the force of repeated experience and sensible confirmation. In the consciousness of the young child, the wings and scales once suggested do arise and remain: its waking states are as novel as its dreams. But as experience grows, mental.

1 Senses and Intellect, chap. xIII. $\$ 5$. 
products become as firmly held or as firmly banished as their nervous correlatives, and the growth on both sides is a growth in inhibition.

It is only when we examine certain classes of states a little off the normal that the force of the principle is fully seen. In cases of "fixed ideas" or monomanias, a single presentation acquires an inhibitive force over the entire content of consciousness. No other subject of thought has any chance of a hearing: it is immediately choked off by the more violent than constitutional rulings of the "speaker of the house." Again, in cases of hallucination and illusion the normal reports of the senses are inhibited in whole or part by the false image which fits itself into the vacant place. Particularly in hypnotic hallucination are the phenomena presented in remarkable purity. The suggestion of a positive object in the room is immediately followed by the sight or hearing of such an object by the patient; the suggestion, which is entirely imaginary, hiding the real objects of the room. The images of the latter are thus inhibited. Eren a negative suggestion, as it is calledthe suggestion simply of the absence of such or such an object-is at once realized. A further important point is well established, namely, that the inhibition of an image takes place only at the points at which it is really inconsistent with the suggested image; in other points the two images coalesce. For example, cases are recorded in which if the patient was told what he saw was the picture of a hat, when in reality it was the photograph of a man, he saw a man wearing a hat; but if some other animal was suggested, the man was not seen at all. Similar coalescence of images occurs in dreams."

To bring these latter cases under the head of real inhibition a further point of evidence is wanting: that 
the normal image is really present at all; that the nervous inhibition has not been so complete as to cut off altogether the physical process corresponding to the normal image. This point is fully supplied by recent observations. The fact mentioned already that the suggested image may coalesce with the image of the reality seems to show that the normal image is really present before the suggestion. Further, it is proved that the patient must recognize the image which he is not to see before he does not see it, or before it is hidden by the suggested image. This is evidence that there is real conflict in representation and a repression of one mental state by another. ${ }^{1}$

A great source of such involuntary and subconscious inhibition is expectant attention or eager desire. What may be called the "scientist's illusion," his tendency to find in his specimens the facts he looks for and desires, often arises from the inhibition of the true images presented, by the vivid representation of the marks his theory leads him to expect. Strong emotions also give an inhibitory force to the ideas which arouse them or toward which they are directed.

\section{§5. The Nervous System and The Unity of Consctousness.}

The functional unity of the nervous system has already received sufficient emphasis. The conception advocated in the preceding pages is a dynamic conception. The parts of the system have meaning only as they are related to each other in a system whose activity as a whole gives value to the activity of the parts in the general life-process. The last analogy, therefore, that concerns us is a gathering up of all the partial analogies with which we have been hitherto concerned. We have not many nervous systems, but one; the laws of its growth

${ }^{1}$ Cf. Binet, loc. cit., p. 139 f., for interesting details ; also for other cases of inhibition of images by optical experimentation. 
are not many, but one; its function is one, its teleological end is one.

So consciousness has not many forms, passive, reactive, sensory, motor, voluntary, inhibitive. These are all partial aspects of a single unitary presence. There is no sensory phenomenon but has its dynamic or reactive side. There is no motor phenomenon in consciousness, but it springs from antecedents of sensibility. There is no voluntary phenomenon, but it rests on both. Consciousness, therefore, is one as the nervous process is one.

\section{§ 6. Organic Theory of the Unity of Consciousness.'}

Maudsley sums $\mathrm{up}^{2}$ this theory in three great points, which may be stated logically thus: First, the brain, as the organ of consciousness or thought, is capable of dual activity, this duality making it impossible for us to look for any unity in consciousness as far as the thought-processes alone are concerned; second, the real unity of self is to be found in the affective or emotional life, which, third, finds its basal principle of unity in the organic unity of the body, i.e., in the nervous system. These points are closely interwoven, and present an account of the mental life to which spiritualists generally take broad exception. It is our purpose to indicate some considerations from a psychological standpoint which tend to show that Maudsley's physiological data do not suffice for the interpretation he gives them, leaving out of account altogether the evidence to be drawn later from volition as a fact of conscious unity.

The facts bearing upon the dual nature of the hemi-

3 This section has been published substantially in Mind, vol. xIv. p. 545, under the title Dr. Maudsley on the Double Brain.

In Mind, vol. xIv. p. 161, art. The Double Brain. For his detailed position, see his Physiology of Mind. 
spheres and the functional interpretation of them in regard to movement which Maudsley gives, are conceded from the outset. It seems to be established that, besides the common functional activity of the hemispheres, that area over which they both have dominion, there is a residuum to each alone, a large body of motor functions peculiar to each respectively ; and that either may dictate the performance of their common function, together with that which is peculiar to itself. It is when we pass on to consider " how the hemispheres act toward one another in thinking," that is, how they are related to each other as respects the apperceptive consciousness and its unity, that the question of psychological interest arises.

I. Absence of Unity in the Motor Consciousness. First Argument: from the separate activity of the hemispheres. In answering this question, Maudsley first cites the case in which we attempt to perform movements involving the separate activity of the hemispheres, as the performance of different movements with the two hands. He says: "If a person who is performing one kind of act with one hand and auother kind of act with the other hand will endeavor to think of both acts at the same moment, he will discover that he cannot do so; although he can execute the different movements simultaneously, he cannot think them simultaneously; he must pass in thought from one to the other, a rapid alternation of consciousness takes place. This alternation, though rapid, is by no means simultaneous; it is distinctly successive, since there is an appreciable pause in the performance of it." After excluding other alternatives, such as the coexistence of different consciousnesses, he concludes that "there remains the supposition of an alternating action of the hemispheres corresponding to the alternating consciousuess." This alternation, he 
goes on to show, gradually yields, on the part of the hemispheres, through repetition and education, to their union in simultaneous activity as a single organ, but consciousness preserves its method of "extremely rapid alteruations." The conclusion, therefore, as respects intellectual unity, is that we find no basis for it in the functional activity of the hemispheres.

Answer. This conclusion may be true, but the analysis it involves of the psychological unity of the states involved is so meagre and false that we cannot take it alone with us in our search for the true principle of unity. By consciousuess in this connection Dr. Maudsley seems to mean attention. It is true that I cannot attend to the two movements at once, that my attention alternates usually even when the movements are simultaneous; but it is not true that I may not be conscious of the two movements at once. Repetition tends to make them elements of a single state of consciousness, just as repetition tends to make the hemispheres a single unit organ. A simultaneous consciousness is not a " distracted or dual consciousness," but an integrated consciousness, a new state whose elements are drawn from previous states. Attention is a state of monoïdeism, but consciousness is not.

Now this integration of states is possible only on the basis of a fundamental intellectual unity as necessary to the mental life as organic unity is to the members of the body in their variety of physical functions. If I move my right thumb to the left, is the movement my only consciousness? Am I not conscious that it is $m y$ thumb, my movement? Are there not unnumbered organic, detached, stray peripheral sensations bound up with the act or with its very thought? And when I shift my attention and move my left thumb to the right, is there a pause in my consciousness of all these things? Not at all ; I am just as conscious of my thumbs, of my 
organic affections, of myself, between the movements or during them. A simple change in my motor experience can in no sense be said to create a pause or break in my consciousness. Each hemisphere, instead of contributing a separate consciousness, contributes an element of content to my single consciousness - a motor element. And further, attention itself as a principle of active unity is dependent upon the complexity of the mental life. The selecting, relating, unifying, disposing function of attention has been so emphasized in recent discussion that it is needless to dwell upon it.

We have thus briefly touched upon three elements of mental unity which analysis seems to give and which demand explanation whatever hypothesis we adopt. First, a subjective reference of all modifications, both sensor and motor; second, the subordination of incidents in consciousness, past and present, to the permanence of consciousness itself, which remains as the background of their flow ; third, the grasping and disposing energy of attention, which is always one. The class of movements hitherto spoken of, i.e., those which are controlled by the hemispheres individually, with no coöperation, bear only upon the incidents and not upon the higher aspects of mental unity.

If the case rested simply with this class of movements, Maudsley might strengthen himself by extending the difference of function not to the two hemispleres only, but to each of the motor areas within each hemisphere. The motor speech-centre, for example, is distinct from the centre for the movement of the lower limbs. We can perform the two functions-speaking and walking-simultaneously, but cannot attend to them simultaneously until a close association is brought about by education. Therefore, it might be argued, motor consciousness is a matter of successive states and lacks unity. From this point of view we have not two brains (centres), but, perhaps, a dozen. But the unity of the mental life, for which the motor consciousness only serves as point of departure, remains quite untouched.

Second Argument: from the conjoint activity of the hemispheres. Maudsley next proceeds to consider those movements in which the hemispheres coöperate; they 
"combine to dictate different movements of the two sides for a common end, just as the eyes combine the different visions of one object." The question is this: "From what higher source do the hemispheres obtain their governing principle of unity? How is it that when dictating different movements they yet have an understanding to work together to a common end?" And the answer is again that the unity of the motor consciousness is an educated unity, and that, like two acrobats, they learn to perform together " by much travail and pain."

Answer. This is true, and its importance can hardly be estimated; but it again must be criticised on the ground of what it leaves out. We are forced at once to inquire: Whose is the "end or aim in view," the "conception or foresight of the act, its ideal accomplishment"? Certainly not the conception of the hemispheres themselves, though the figure of the acrobats would lead us to think so; for how could such a conception be acquired by the hemispheres before the action had been actually performed? And if thus acquired, how could it be intercommunicated without a central bureau of consciousness where the progress of the coördination of movement might be apprehended and recorded? The conception which precedes all effort at motor execution is a fact of unity, higher mental unity, an ideal unity of the motor consciousness to which the complex activity of the motor apparatus is to be reduced by long and wearisome effort. Here, again, is the outgoing of the attention in its relating and efficient activity, grasping the whole while itself is one, relating the many in an ideal which is one, and reducing the many to the unity of the foregoing ideal plan. Here, as in the former case, we find no fault with the account of what takes place in and for the motor consciousness; but we cannot see how this consciousness can be considered for itself alone in independence of the higher thought-consciousness in 
which alone the idea of motor coöperation germinates and bears fruit. And our conclusion is that mental unity is something independent of coördinated movements.

The other figure which Dr. Maudsley uses in this connection makes the case still plainer. He says the hemispheres are related to each other, in such coördinated movements, as the eyes are in binocular vision, their early binary images being reduced by experience to a unitary perception. But the eyes do not accomplish this themselves. The ideal plan of vision precedes all reduction. Let us suppose the eyes to be the seat of consciousness. Now, either each eye has its consciousness, or there is a single consciousness for both eyes. If each has its own consciousness, neither eye could be conscious of its disagreement with the other and their results could never be reduced to unity except by accident. If there is one consciousness for both eyes, it is in virtue of this unit consciousness that a unit perception is attained and not from anything in the eyes themselves; that is, it is only through the interpretation of a unit consciousness, which renders both images as such possible, that they can be reduced to the form of vision which is their ideal conception.

The mental unity to be explained is something more profound than the simple consideration of the motor consciousness would lead us to expect; it remains to see whether the organic solution offered by Maudsley is adequate.

II. Mental Unity entirely Affective and (III) explained by Nervous Unity. The two great questions here involved are these: Is the "unity of the intellectual life based upon the unity of feeling," and "this again upon the unity of the organic life"? These questions are so 
comprehensive and far-reaching that only a few general considerations can be advanced in this connection.

Answer. 1. The same line of argument by which Dr. Maudsley and others' prove the absence of unity in the motor consciousness applies with undiminished force to the affective consciousness. Can we attend to two simple sensations in two peripheral organs at once, say a taste and the pain of a wound in the hand? Not at all. The case is just the same as when we try to perform two movements on different sides at once. There is the same alternation of attention until the sensations become united in a single attention-complex. The isolation of single affective states in our adult life is open to the same charge of psychological atomism as has been found attaching to the similar isolation of motor states. Indeed, simple feelings of movement are themselves affective states, being simply intensive, and the argument from them applies to all states of the class. The feeling of effort which is bound up with movements is quite distinct in its nature, and seems, as has been said, to indicate a higher plane of intellectual unity, which the theory in question leaves quite out of account.

2. We may well notice that neither the manifoldness nor the unity of feeling could be apprehended as such in the absence of a circumscribing consciousness which, through its own unity, takes it to be what it is. Suppose we admit that at the beginnings of life the inner state is simply an undifferentiated continuity of sensation; what is it that feels or knows the subsequent differentiation of parts of this continuity? It cannot be the unity of the continuity itself, for that is now destroyed; it cannot be the differentiated sensations themselves, for they are many. It can only be a unitary subjectivity

Horwicz. 
additional to the unity of the sensory content, i.e., the form of synthetic activity which reduces the many to one in each and all of the stages of mental growth. The relations of ideas as units must be taken up into the unit idea of relation-to express what modern psychology means by apperception.

3. It is difficult to see how the higher intellectual unity, of which we have spoken, can find its basal principle in the organic unity of the body. Admitting, with Maudsley, that mind is a matter of organization, the progressive organization of residua, we never are able to go outside the unity of conscionsness to find mental residua. Indeed, there can be no such thing as a residuum, except as it is the same in nature as that of which it is a residuum: and admitting further that the body is also an organization and an organization which proceeds in the most intimate and progressive parallelism with that of mind, we are yet unable to make mental organization a function of physical organization until these propositions are established: (a) That the law of the organic growth of mind finds its proximate ground in the growth of body; that is, that the methods of nervous integration run also into mental integration. Now, as a fact, the great principle of mental organization, selective synthesis, finds no apparent counterpart in physics; its higher products find no objective realization in the syntheses of physical organization. It seems, as Lotze says, to be unique. (b) That there is a correlation of mental and physical force, a principle everywhere assumed by Maudsley and others, but nowhere proved. (c) That mind in its progressive organization does not exhibit autonomic energies of its own, constructs no ideals of its own; and further, that the two aspects of unity, physical and mental, are not themselves members of an underlying principle to 
which they are both secondary and which may be mind.'

The conclusion thus reached may be further strengthened by an appeal to the psychology of volition. ${ }^{2}$ Although, for clearness of argument and criticism, this consideration has so far been omitted, we shall find the crucial evidence of mental unity in the unity of volition. When the organic theory of unity has successfully fulfilled the requirements already exacted of it, it will be time to demand that it give solnething like adequate recognition to the outstanding facts of the volitional life. Prof. Bain, at any rate, goes with us in locating the unity of consciousness in the active or "executive" function."

Recent endeavors to reduce the unity of consciousness are based on pathological cases in which consciousness is split up into two or three, or subsidiary consciousnesses are found corresponding to partial expressions of the unity of the organism." But these cases are not conclusive as long as the function of consciousness is not accounted for. Each splitoff consciousness does something which nothing else in the world can do. Let us manipulate consciousness as much as possible by manipulating the nervons system, yet as long as each piece of conscionsness acts as only consciousness can act, with a unity of its own, we have a thing sui generis : for action, function, is the only test of a thing.

\section{§ 7. Heredity and Consciousness.}

The obscurity which envelops the biological problem of heredity grows deeper still when we seek to bring the phenomena of mental heredity under any general statement. We may say that there is such a thing as mental heredity ; that is, we may expand the conception of conscious integration or mental growth beyond the range of

\footnotetext{
${ }^{1}$ On this discussion see the acute observations of Dumont, loc. cit., chaps. v. and vi.

Chap. XVI, below.

${ }^{3}$ Emotions and Will, Appendix A.

- Maudsley, Ribot (Diseases of Personality), Binet (arts. in Revuo Plilosophique): the most remarkable facts are recorded by Janet, Automatisme psychologique.
} 
individual experience into race experience. But in so doing we must bear in mind that mental growth depends upon physiological retention. Consequently, psychological heredity, strictly considered, would not include the inberitance of mental images or mental products of any kind: we have found such a storing-up capacity in the mind to be mythological. But mental inheritance would consist in a tendency to stronger and better developed mental function. As far as our knowledge goes, it is this aspect of mental heredity which actual observation confirms. Parent and child have in common strong or weak logical ability, keen or blunt sensibility, strength or irresoluteness of will; but we find no reference of their logical ability, or of their peculiar emotional or volitional disposition to any particular class of objects.

Yet we find in animals remarkable cases of such particular forms of inheritance. The whole class of animal instincts have such particular reference. How can we explain the chick's congenital fear of the hawk, except by supposing the inheritance of a particular reaction stimulated by the presence of the hawk? In such cases, however, an organic explanation suffices. Here the inheritance, besides involving the broader and more massive features of the parent's nervous constitution, extends as well to particular habitual reactions; and these latter are so consolidated even before experience that they respond to their appropriate stimuli. Because the child's limbs show an alternating reaction when the soles of the feet are stimulated, we would not say that the child inherited mental images of the muscular movements involved in walking.

Further, the limit which our present knowledge imposes upon the principle of nervous heredity applies also to mental heredity: such inheritance is limited to cases of immediate descent. Only where possible gen- 
eration demonstrates nervous heredity have we any ground stronger than analogy for postulating mental continuity of development. The question of mental ovolution is a vital one in present philosophical discussion: and in this generation important contributions to its solution are being made. This reference to it, however, is sufficient to indicate the point at which it would have its psychological importance, and beyond such an indication empirical psychology has no right to go.

On the nervous system as organ of consciousness, consult : references in Senses and Intellect, pp. 34, 68, and 114 ; Bastian, Brain as an Organ of Mind: James, Principles of Psychology, vol. I. chaps. II-III ; Bain, Mind and Body; Maudsley, Body and Mind and Body and Will; Herzen, loc. cit.; Ribot, Diseases of Personality ; Lewes, Problems of Life and Mind, $3 \mathrm{~d}$ series, I and II ; Horwicz, Psychologische Analysen, Theil I. 2d Absch., and Theil II. 1st half, bk. I; Bouillier, La vrai Conscience, chaps II-IV and (unity of) VII ; Brentano, Psychologie, I. bk. II. chap. Iv; Paulhan, L'activité cérébrale et les éléments de l'Esprit, pt. Ir. bk. II (inhibition) ; Calderwood, Mind and Brain, chaps. vin-Ix; Wundt, Menschen und Thierseele, 5-9te Vorl.; Ferrier, Functions of the Brain, 2d ed., chap. xII ; Höffding, Outlines of Psychology, II ; Romanes, Mental Evol. in Animals, chaps. I-vII ; Sergi, Psych. physiologique, liv. II. chaps. I-III, and liv. III. chap. I ; Münsterberg, Beiträge zur experimentelen Psychologie, I, Einleitung; Fechner, Elemente der Psychophysik, 2 d ed., pp. 377 ff.; Ladd, Elements, part II. chaps. I-III and IX-X, and part III. chap. III ; Spencer, Princ. of Psychology, I. part I. chaps. I-VI, and part v. chap. vIII ; Bain, Emotions and Will, pp. 539 ff. and Appendix A; Lotze, Metaphysic, bk. III. chap. $\mathbf{v}$ (inhibition) ; Symposium in Brain, xI. pp. $361 \mathrm{f}$.

Further Problems for Study :

The nervous accompaniments of the attention;

Inhibition in psychology ;

Mental heredity;

Mental evolution in animals and man. 


\section{CHAPTER III.}

\section{NATURE AND DIVISIONS OF SENSIBILITY.}

\section{$\S 1$. Nature of Sensibility.}

Deflnition. The transition now made from the nervous basis of mind to the phenomena of consciousness leads to topics more properly psychological. The term sensibility has been used heretofore as almost synonymous with consciousness; at least the assumption has been made that when consciousness is once reached, sensibility or feeling is its primary and most general characteristic.

Empirical observation justifies this assumption. Our final interpretation of all mental facts in common life is in terms of personal feeling. How do $I$ know that $I$ am willing a given act of conduct? Because I feel the act of will. My immediate ground of confidence is a qualitative state of being affected, which I have learned to distingu ish in my experience under the name will. How again do $I$ reach the assurance that I am thinking and not willing? By a similar awareness of feeling. I am affected in the way which I call thought. The original awareness of consciousness, therefore, is an affective state, and as consciousness is the form of all subjectivity, so sensibility, feeling, is its first content.

If this be true, we would expect to find feeling everywhere in the mental life. It would be a more or less prominent accompaniment of all possible states of consciousness. This view, though generally admitted by psychologists, is only partially accounted for on many 
of the theories of sensibility; it will become clearer after the examination and description of the various classes of feelings.

Looked at from this aspect of universality which sensibility presents, from its logical primacy among conscious states, and from its peculiar subjectivity in opposition to the objective reference of intellect and will, we may arrive at a general definition of feeling: feeling is the subjective side of any modification whatever of consciousness, or it is the simple awareness of the unreflecting consciousness.

The force of the position here taken will pe more evident after the general discussion of emotion, especially since two great theories of the emotions-the physiological and the Herbartian-agree in denying to the emotions ultimate qualitative distinctions. Here as elsewhere our final position must rest upon the facts of consciousness.

A description of pure sensibility has already been attempted above in speaking of passive consciousness, ${ }^{1}$ and it is unnecessary to repeat it here. 'The fact of feeling is so clear in our common experience that no more exact definition would be needed if it were possible. What we mean by my consciousness in opposition to your consciousness sums up feelings. You can know what I know and you can will what I will, but you cannot by any possibility feel what I feel : this is subjectivity, this peculiar and unapproachable isolation of one consciousness from another.

Most General Mark of Sensibility. A distinction has already been drawn between common or general sensibility, and the more particular kinds of affective modification which we call sensations. The latter belong, speaking. generally, to the more differentiated portions of the nervous system provided with special end-organs. Of these we may also say that they have their seat in particular brain-areas, though it is likely that this will not. prove to be a permanent mark of distinction when our

' Chap. II. § 3 . 
knowledge of the brain-functions has become more complete.

In common feeling, ${ }^{1}$ therefore, are included all modifications of sensibility which do not come under any of the classes of special sensation. Stated thus negatively, the way is open for the differentiation of this great fund of sensibility into as many particular divisions as psychological analysis may be able to discover.

When such analysis has been pushed to its extreme and qualitative differences in sensibility have been pointed out as far as may be, the point of interest then remaining has reference to the most general mark of sensibility itself, the common element beneath all its concrete forms. What is it that brings the special as well as the organic sensations, the vaguest feelings of physical unrest as well as the acutest pang of an exposed nerve, all under the common name feeling? Such a common mark cannot be found in the simple fact of consciousness, for other mental states, intellectual and volitional, are also conscious. Yet it must be something that attaches primarily to consciousness, since sensibility as a form of subjective modification presents closest analogies with the nervous basis of consciousness. Without anticipating a later justification of this decision, it is plain that this most general characteristic of sensibility is pleasure and pain.

Pleasure and pain, therefore, may be set apart, at least for convenience of exposition, from the particular mental phenomena which they accompany. If pleasure and pain be truly designated as the most general characteristic of sensibility, then no mental state whatever would be entirely neutral as respects pleasure or pain. Yet in the great complexity of the developed mental life, where cross-currents of feeling interfere with one

1 German, Gemeingefühl. 
another and neutralize the effects of one another, it is quite possible that pleasure and pain may not enter as an outstanding feature of consciousness : indeed such a real neutrality as regards pleasure and pain may be attained in the bosom of sensibility, that while feeling remains, its tone is without positive conscious coloring. This possibility, however, must wait upon the further consideration of pleasure and pain.

It is, therefore, safe to speak of the feelings as phenomena of pleasure and pain. If there be exceptional cases, it is because such states are rich in tone, not because all tone is absent. This is an easy criterion and the one generally recognized by psychologists, the question of neutrality being mainly of theoretical interest. ${ }^{1}$

As concrete facts, however, pleasure and pain are always elements added to some conscious content. It is in this aspect that they are described as tone, the states of which they are the tone being more or less exactly discoverable. According as they are thus realized as one element in a complex, they give the name complex sensibility to the complex; in contrast to simple sensibility, which is pleasure or pain abstracted from all such complexity. ${ }^{2}$

1 See Chap. XI. $\$ 4$.

"Some writers find a something called "feeling" apart both from qualitative sensations and from pleasure and pain; Ladd, loc. cit., p. 514 ; Nahlowski, loc. cit., p. 131. I am unable to find any such pure unqualitative feeling, either in sensation or emotion. Wundt's later position seems more true, namely, that sensation and sensuous feeling are one and the same thing. 


\section{§ 2. Divisions of Sensibility.}

In view of the foregoing, states of sensibility may be thrown into the following table:

Sensibility:

I. Lower, or Sensuous, Sensibility.

a. Complex : sensuous feelings.

1. Common sensuous feeling.

2. Special sensuous feelings : sensations.

b. Simple: sensuous tone.

II. Higher, or Ideal, Sensibility.

a. Complex : ideal feelings.

1. Common ideal feeling.

2. Special ideal feelings: emotions.

b. Simple: ideal tone.

Accordingly we turn at once to sensuous feeling. The term feeling as now used signifies any affective state whatever, the general sign or mark of feeling being its tone of pleasure or pain. This usage, which has its justification here, is seen to be the same as that tacitly adopted in the former volume throughout.

On the nature and classification of feelings, consult: Mercier, Nervous System and Mind, pp. 280 f., and Mind, IX. 325, 509, and $\mathrm{x} .1$; Nahlowski, loc. cit., pp. 41 f.; Hamilton, Metaphysics, Lects. XLI and XLV; Biunde, Empirischen Psychologie, II. \$210; Herbart, Lehrbuch d. Psychologie, $\$ 98$; Kant, Anthropologie (ed. Kirchmann), p. 138 ; Schneider, Thierische Wille, pp. 95 ff.; the Psychologies, in locis ; Hodgson, Theory of Practice, bk. I. chap. II. part I ; Horwicz, Psychologische Analysen, Theil II. 2d half, bk. I, and pp. 521-24; Drbal, Psychologie, \$s 106-110; Sergi, Psych. Physiologique, liv. IV. chaps. I, II ; Paffe, Considérations sur la Sensibilité; Dumont, Théorie de la Sensibilité, part 1 and part II. chap. 1 ; Sully, Outlines, chap. XI ; Ladd, Elements, part II. chap. IX ; Spencer, Psychology, part Iv. chap. viII ; Bain, Emotions and Will, pt. I. chap. III, and Appendix B ; Brown, Philos. of Human Mind, Lect. 52. 


\section{SENSUOUS FEELING.}

\section{CHAPTER IV.}

\section{SENSATION AND COMMON FEELING.}

\section{§ 1. Sensations as Forms of Sensibility.}

The dual character of sensation as presentative and affective has been emphasized in a former chapter. The presentative side was held up for prominent review in the discussion of the intellect, since developed knowledge has its point of departure in sense-knowledge. Yet even there, occasion was taken to point out the more striking affective quality of sensations considered as the first things of mind. Subsequent reflection disclosed the fact also that the results of analysis are one with the results of simple observation; the so-called presentative elements were seen to be due to reactions of the apperceptive principle on hypothetical affective ele. ments. ${ }^{2}$ This means that if we were able to abstract all traces of apperception, as a process of relation, from consciousness, the data left would be entirely affective, and the distinctions among phenomena-if any remainedwould be due to the primary properties of feeling. In other words, to be perfectly clear, there seems to be no original knowledge antecedent to the knowledge which is constituted by the reaction of consciousness as apperception.

'This statement anticipates the analysis of the idea of force : the earlier analysis of space and time, the other "presentative elements," justifying the statement apart from force. 
It has also been claimed, in an earlier connection, that while these states of feeling called sensations are one in their peculiar nature as affective, they are different from one another as respects quality. The theory of the "Unity of Composition of Mind" has been found to be inadequately supported. Sensations, that is, have qualitative marks or distinctions inside the conception of sensibility; and while we are able to reduce the nervous functions which accompany them to a single conception, no such reduction of sensations seems forthcoming.

A very brief review of the qualitative distinctions among sensations, accordingly, may be found sufficient here. Disregarding the presentative element, so called, the following classes of special sensations may be pointed out, the rule of choice being the presence of distinct end-organs: from the eye, sensations of lightintensity and of color; from the ear, sensations of sound-intensity, timbre, and height; from the tongue, sensations of taste ; from the nostril, sensations of smell ; from the skin, sensations of contact and temperature; from the muscles, sensations of movement.

It may be well also to recall at this point the properties of sensation, quantity, quality, duration, and tone. With the first three conceptions we have already busied ourselves; the fourth will have abundant claim to recognition as we proceed.

A further analogy in favor of the "Unity of Composition" or "Mindstuff" theory might be found in the conception of the nervous system advanced in the preceding pages. If the nervous system has resulted from the integration of original nerve-elements of a single kind, is it not likely that consciousness in its complexity has had a corresponding development under a single principle of integration? If this analogy applies at all, it applies to the development of consciousness after consciousness is given : that is, the unity of consciousness must be presupposed, as we have seen above. But after we enter consciousness, we find a principle

${ }^{1}$ Senses and Intellect, chap. VII. $\$ 4$. 
of apperception to which there is no analogy in physiological integration. Consequently this argument would apply only to differentiation in the bosom of pure sensibility, being restricted both above and below. But the attempt to reduce sensations to a unit form of sensibility has been examined under the "'Theory of Unity of Composition." So every aspect of this analogy has had careful attention; whether it still holds or not, in spite of this adverse treatment, is another question.'

Relativity of Sense-qualitios :' Contrast. While we do not find ground for the reduction of sense-qualities to a single mode of sensibility, yet we do find a series of phenomena which show that there is no fixed typical sensation of each quality; but that all determinations of quality are to a degree relative distinctions among many " moments" in consciousness. These are the socalled phenomena of contrast. The general statement of fact is this: Any sensation (color, sound, taste) which occurs after or with other sensations (colors, etc.) is different from what it would have been if the other sensations had not been present, or if the other sensations had themselves been different: the variation, however, is within the same sense-quality.

In the domain of the special senses, such effects of one sense-quality upon another may be subjected to experimental determination by psycho-physical methods. The phenomena of color-contrast are the richest and best understood class of facts. In general, color-contrast means that when part of the retina is stimulated to react to a particular color, there is a tendency of other portions to react to the complementary color. For example, the so-called "Meyer's experiment" may be cited: put a scrap of gray paper on a colored (red)

'Since the section on the "Unity of Composition" theory was written, Prof. James has published an acute criticism in substantial agreement with it: Principles of Psychology, vol. I. chap. vI.

, On the relativity of emotions, sec Chap. X. $\S 1$. 
background, and spread over the whole a sheet of white tissue ; the gray scrap will now tend to assume the color complementary to the background (green). Recent research has developed a number of interesting optical phenomena of this class. Stumpf ${ }^{2}$ has recently discovered that the pitch of a note is modified by the occurrence of another note of a different pitch, in such a way that the interval between them is lessened. Striking contrasts are also easily demonstrated in color, light, and tone intensities. Contrasts of temperature are also easily brought about. Cold water feels colder if the hand is just from warm water. Differences in temperature of the two hands lead to exaggerated differences of sensation when they are plunged together into two vessels of water of the same temperature. Contrast is called simultaneous or successive according as the rival sensational qualities occur together or in succession.

Two theories of sensational contrast have been advocated, one called the "psychological," according to which such contrasts are errors of judgment or synthesis, the actual sensations themselves having fixed and unaltered qualities. This has been held by Helmholtz," and has been used to support the theory of "unconscious judgment." The other, the "physiological theory," holds that contrast-effects are due to complex conditions of stimulation. The different color-stimuli, for example, are not reported separately to consciousness; but only their united effect is operative in the optical centre. Consequently, what we have is a case of summation or fusion of stimuli, not of comparison and judgment of sensational atoms. This latter theory is now com-

1 The white sheet over the whole is necessary to obscure distinct lines of separation between the colors beneath: if such distinct boundary-lines are exposed, the contrast-phenomena disappear.

Tonpsychologie, II ; see Mind, xvI. p. 279.

3 Physiological Optics, pp. $388 \mathrm{f}$. 
pletely victorious, principally through the brilliant experimental work of Hering.'

Simultaneous contrasts are seldom free from the influence of successive. And successive contrasts are more striking and general. Both of these observations are explained by the fact that successive nerve-stimulations are more readily distinguished from one another, and give room for the influence of fatigue from one sense-quality before the next is experienced. 'That a more unused function will tend to monopolize consciousness and extinguish a more used function-this may be called the law of nervous fatigue as regards contrast.

Conclusion on Sense-qualities. It seems reasonably safe to conclude that there are well specialized nervous functions which correspond to the great differences of quality in sensations : this is shown by the fact that the differences are stable; that the senses are largely independent of one another in their activity; that each such function has normal minimum and maximum activities which give original degrees of intensity in consciousness. But within these limitations, both qualities and intensities are subject to the law of relativity as well by reason of nervous summation as of mental synthesis. ${ }^{2}$

Relation of Sensations to Knowledge. Sensations, in the sense of purely affective "moments" in consciousness, seem to occupy, in view of all that has been said, an anomalous and, in the main, fictitious position. We have found that the simplest presentative aspects of sensation, space and time ${ }^{\prime}$ arise from the synthesis of apperception. Differences of intensity (quantity) and quality then remained. But we found also that differ-

'Pfüger's Archiv, xuI. p. If and 360f; also xLvir. Hefte 4 and 5. A brief exposition of the facts and theories is given by Delabarre in James's Princ. of Prych., II. pp. $13 \mathrm{f}$. See also Stumpf, Tonpsychologie, vol. Ir.

Sce next paragruph.

Senses and Intellect, in locis. 
ences in intensity were relative (Weber's law), ${ }^{1}$ i.e., that intensities are not constant, but vary as they are distinguished from lower or higher intensities. We now find that qualitative differences are also relative (law of contrast), i.e., that a sense-quality varies as it is brought into juxtaposition with other sense-qualities. But as distinguishing (differentiation ${ }^{2}$ ) is an apperceptive function, all distinctions whatever among sensations, in consciousness, are due to the synthesis of apperception. So much on the side of consciousness of differences among sensations : the reactive and voluntary consciousness.

But there are, we find, differences of which we are not conscious. Sensations, we saw, may be analyzed (sound, sight, etc.). This we found to take place by the breaking up of a nervous process resulting from summation." Intensities, again, depend upon the relative strength of stimuli (Weber's law); this also was found to be due to the compounding of nervous excitations." Further, sensations are subject to unconscious contrasts in quality, which is also explained by nervous summation(Hering's experiments). Hence there are sensations arising from differences (summations, overlappings, subtractions, etc.) among nervous processes, sensations due peculiarly to such complex processes, and not to the union of the various sensations which the elements of these nervous processes would have severally produced. Such supposed sensations are not distinguished at all : pure pleasure and pain, if they could be realized after birth, would be such : i.e., passive consciousness.

But the latter we never find-except hypothetically in the lowest forms of life. Wherever we actually find consciousness we find also reaction, and consequently synthesis. Feelings are always either feelings from or

${ }^{1}$ Senses and Intellect, in locis.

Ibid., Chap. IV. § 2 .
3 I bid., Chap. IV. $\$ 2$.

4 Ibid., Chap. VII. $\$ 5$. 
due to (not of) external or internal relations, or feelings of internal relations (emotions). The former are either sensuous or ideal, the latter always ideal. We may, therefore, conclude that there is a synthetic element in sensation itself, that thought is implicit in the earliest reactions of consciousness. And sensations considered as atoms, as the materials given in consciousness to be worked up, or to work themselves up, do not exist: the sensation is itself the outcome of consciousness, which is a function, not an empty theatre or a blank tablet.

Feeling and knowledge are, finally, only two sides of the original fundamental fact, consciousness, which is a dynamic creative thing in relation to its own content. It begins by creating blindly, impulsively, under the lead of cerebral processes: this is feeling. It ends by creating with prevision, selection, thought: this is knowledge.

To illustrate: an infant's first visual sensations, say of its father, are already felt to be different from the muscular feelings which preceded, these from earlier feelings, etc., back to the beginnings of consciousness itself, i.e., reaction, giving felt differentiation, space-relations, time, memory. He next sees a stranger, and has besides the father-sensations again, other elements due to differences in the two men: these latter arise from relations in consciousness, but are not of them. The child does not know there are two men, it only feels a difference. Later the infant learns to distinguish the men, and has a new feeling of difference or similarity, which could only come after the relation in consciousness is a conscious act of relating, i.e., is knowledge.

The current theories of the relation of sensation to thought may be thrown under the following heads :

1. Affirms definite fixed atomic sensations, which are the material either of association (associationists, Hume) or of 
rational construction (idealists, Kant, Green'). This is refuted by the phenomena of contrast.

2. Affirms that there are no sensational atoms, that contrast-phenomena are due to physiological summation, as is also thought (Maudsley, Ribot, Sergi, Münsterberg). This is refuted by facts of apperception. Physiological summation would simply give larger atoms in place of thought, not intelligent distinction, selection, and relating of elements.

3. Denies atoms, makes contrast-phenomena due to summation, which is accompanied by conscious thought with native categories of objectivity, space, etc. (Höffding, ${ }^{2}$ James). 'This account forfeits unity of treatment between thouglit and sensation-making thought a relating process depending upon sense-material, but finding in sensation a ready-made cognition of relations which cannot be further analyzed. We may at once ask : If sensation gives knowledge, why does it not operate under the rubric of knowledge, i.e., synthesis? This criticism can be avoided by grounding native cognition either in inherited nervous coördination (Spencer), or in formal apriorism (Kant); but the first alternative denies the synthesis of higher thought (2. above), ${ }^{3}$ the latter carries us back to atomic sensations to furnish material for a priori forms (1. above).

4. The theory of the text denies atoms, affirms nervous summation, as demonstrated by Hering, to account for contrast and general relativity, asserts mental synthesis present in all sensation, but at first implicit, felt only ; this synthesis further becomes explicit in knowledge and gives new feelings called ideal, intellectual, etc."

This is the only theory which the writer is able to reconcile with the facts adduced by the physiologists,

${ }^{1}$ Also, among the physiological psychologists, Helmholtz and Stuinpf.

2 Outlines of Psychology, pp. 113-17. Höffding's position is not clear. He denies the distinction between sensation and thought, making contrast-phenomena a matter of synthesis (p. 116). But he also finds nervous summation to be the ground of contrast (p. 114). These positions, in both of which I follow him, can be reconciled only by making the nervous summation at once the physical basis of an implicit thought-synthesis.

${ }^{3}$ Munsterberg, who accepts this alternative, brings the criticism of duality of explanation against Wundt. Beiträge, Heft 1 ; Einleitung, pp. $42-46$.

4 I.e., Emotions, Chaps. VIII and IX. 
and at the same time not do violence to the synthesis of thought. Granted a dynamic interplay of energies in the brain; its function at any time is a resultant of tensions adjusted relatively to one another (summation and inhibition). Granted, on the other haud, consciousness sedent in the brain; its present function is the outcome of intensities, qualities, etc., relatively adjusted to one another in it. Now, if the nervous basis of thought is -as physiology assures us-an advance over that of sensation only in complexity of cerebral coördination, then we would expect that thought would be an advance over sensation only in degree of conscious coördination. The great assumption is made in making consciousness a new kind of coördination in the first place-in getting space, time, quality, etc. Why, when this is done, demand a new concession to account for the synthesis of thought? The ultimate question is, therefore, between an apperceptive and an associative-a cause and an effecttheory of the form of consciousness in relation to its content. ${ }^{1}$

The function of synthesis or coördination in the simplest presentations of the external world is seen in the phenomena of psychic blindness mentioned above. ${ }^{2}$ The brainless creature has his sensations, his data, localized and differentiated, but has lost their meaning, their connection with one another and with himself. The same in true is cases of word-deafness and wordblindness-instances of psychic blindness restricted to one class of sensations. A patient is often able to see a written word and reproduce it, but is blind to its meaning; or he hears the word and repeats it, but is deaf, in turn, to its meaning. He is obliged to learn its connotation and legitimate usage, as the child does in

${ }^{1}$ See the admirably clear way in which Munsterberg joins this issue, loc. cit., Einleitung, especially pp. 34-40.

${ }^{2}$ Chap. I. $\$ 4$. 
the first place. Here many of the syntheses or relationships which we designate by the word "meaning" have been stripped from the sensations by brain-disease or accident.

The various factors involved in ordinary qualitative sensations may be shown in the following table-the process over $b$ (as related to those over $a$ and $c$ ):

Nervous Integration.

\begin{tabular}{|c|c|c|c|}
\hline Consciousness. & Passive Consc. & Reactive Consc. & Voluntary Consc. \\
\hline Feeling. & $\begin{array}{l}\text { Pure Feeling } \\
\text { (hypothetical). }\end{array}$ & $\begin{array}{cc}\text { Feeling arising } & \mathrm{Fe} \\
\text { from Relations. } & \text { ti }\end{array}$ & $\begin{array}{l}\text { eelings of Rela- } \\
\text { tions, Emotions. }\end{array}$ \\
\hline Knowledge. & None. 节 & $\begin{array}{l}\text { Distinctions of In- } \\
\text { tensity, Quality, } \\
\text { Space,Time, Sen- } \\
\text { sation, Percep- } \\
\text { tion, Memory, } \\
\text { Fancy. }\end{array}$ & $\left\{\begin{array}{c}\text { Active Imagi- } \\
\text { nation, } \\
\text { Thought, Ideal } \\
\text { Construction. }\end{array}\right.$ \\
\hline
\end{tabular}

Extensity of Feeling. In enumerating the presentative properties of sensation, extensity or massiveness was distinguished. ${ }^{\prime}$ It is the difference, for example, between the sensation of touch from one inch of the skin and from two, between the immersing of one hand and both in water, etc. This feeling of volume in sensation, which seems to attach to most sense-qualities, was quoted in that connection because it seems to present analogies with the spacial property. That it is found equally in connection with spacial and non-spacial senses, ${ }^{2}$ however, seems to be sufficient proof that it is not an immediate datum of space-knowledge, as some' would have it. It is probable that distinctions of extensity are as fundamental as those of intensity, and that they are due

${ }^{1}$ Senses and Intellect, Chap. VIII. \$ 5.

2 Stumpf finds original extensity in sound-tones.

${ }^{3}$ Argued with great ability and force by Professor James, Psychology, vol. II. chap. $\mathrm{xx}$. 
to the form of implicit synthesis which we have found present in all seusation: further, it is probable that they represent one of the first reactions of consciousness upon a nervous arrangement which has been perfected through former race development and inheritance.

Ward' points out the fact that increase in intensity usually carries increase in extensity with it. A loud sound is a large, voluminous sound. While this is not always true--for example, fine acute pains are exceptions-yet at a maximum intensity there is always a feeling of an overflow, or bursting forth of the energy of sensation beyond its province. When we find that this is also true of feeling and its expressionthe more intense the emotion, the more diffused and convulsive its muscular reaction-we are led to look upon extensity as an indistinct consciousness of the extent, number, and degree of interaction of the nervous elements involved in the sensation or emotion in question. We have already sought to explain the association of sounds with colors, and other similar anomalies, by such a possible interchange of tensions between nerve-areas of great ordinary specialization. Thoroughly in line with this position is Ward's speculation that one extensity-feeling originally embraced all the partially differentiated sense-qualities.

\section{§ 2. Common Sensuous FeElina.}

Common feeling has been marked off as the mass of mixed sensibility which is not included under the great classes of special sensations. It is the fund or reserve of our affective nature from which the special senses seem to have sprung. Apart from the tone which all feeling exhibits, certain farther lines of division may be drawn which enable us to classify roughly the more prominent aspects of common sensuous feelings. As arising from the general vital state of the organism as a whole, such common feeling is called concesthesis. 
Divisions of Common Feeling. ${ }^{1}$ Such a division is based upon the physiological differences to which we would expect some conscious counterpart. The great organic processes of the body go on under the lead and control of automatic nerve-reactions; a body of nerves are delegated to this post of function : there are accordingly organic feelings, the subjective indications of organic health or disease. Again, the periphery of the body is supplied with a mass of fibrils of incalculable delicacy and number which have no representation in the list of special sensations : accordingly a great variety of more or less distinct forms of sensibility seem to originate in the skin and are called cutaneous feelings. Further, consciousness of movement, the so-called motor consciousness, is found on examination not to be simple. It involves an exceedingly complex nervous apparatus, both central and superficial; and all the forms of sensibility which pertain to muscular movement may be designated by the general name muscular feelings. And, finally, the nervous elements are themselves endowed with sensibility. Besides reporting the forms of stimulation which reach the organs with which they stand in immediate connection, the nerves report a variety of conditions to which they are themselves directly sensitive. All such modifications of sensibility may be called nervous feelings.

While not claiming for this division any merits as to final accuracy or exhaustiveness, it seems to meet the demands of convenience and to cover the most obvious phenomena of this category. Certain questions as to further determination will arise below, such as the distinction of muscular from cutaneous, and electrical from nervous feelings; but each such point of doubt, if it be of sufficient importance, can be held over for solution in a more special discussion.

Such a division, it may be further said, gives a degree

${ }^{1}$ See an exbaustive discussion and division by Horwicz, Vierteljahr . schrift für wiss. Plilosophie, IV. 3 ; resumed by Sully in Mind, vI1. 302. 
both of specialization and of differentiation to these phenomena and recognizes, at least in the mature consciousness, a vague localization in regions or organs of the body. The former is true to an unexpected degree of some of these states, especially those called cutaneous. Itching and tickling sensations, for example, have subjectively as much right to be called special as sensations of temperature have. The quality of localization is also quite plain in certain of these classes, and may grow in clearness with experience. The conclusion is therefore strengthened that the accident of a known end-organ is not a safe criterion of distinction.

The normal condition of any one of these seats of sensibility may be contrasted with the abnormal condition, on the one hand, in which the feeling peculiar to it is absent, and, on the other, with that in which this feeling is exaggerated and intense. The former is called ancesthesia, and the latter hypercesthesia. Thus tactile or muscular anæsthesia and hyperæsthesia are conditions of the absence or exaggerated presence, respectively, of the feelings normal to the skin or muscles. The absence of pain, also, in a part where it is present when the usual conditions of pain are realized, is called analgesia.

I. Organic Feelings. The organic feelings were cited in an earlier chapter as typical phenomena of the affective class. They include any consciousness we may have of the massive internal organs of the body. Digestive, respiratory, alimentary, sexual feelings, whether they denote healthful or diseased conditions, may all be put in a single class. The detailed enumeration of the phases of organic feeling is unnecessary. Among the most prominent and important of such feelings, however, we may mention the organic needs, hunger, thirst, air, sleep, exercise, etc., and the large class of feelings connected with the circulation of the blood and the activities of glandular tissue, such as congestion, throbbing, faintness, ete. The pain accom- 
panying these feelings is massive and may be very severe.

II. Cutaneous Feelings. In connection with the skin an enormous variety of feelings are reported in consciousness. Of these, two general classes lay claim to special end-apparatus, sensations of touch and temperature. Besides these, the more definite feelings having their stimulation in the skin are those of itching, scratching, flesh-crawling, tickling, and feelings of the sharp, blunt, hard, soft, rough, smooth, coarse, sticky, damp, dry, oily, etc. Nothing more can be said at present of these forms of sensibility. They are present in greater or less intensity and delicacy wherever the skin is normal. Here also a careful note must be made of the presence of pleasure and pain ; that is, such feelings have tone.

III. Muscular Feelings. The sensuous feelings connected more nearly or remotely with muscular activity present characteristics which forbid the peremptory treatment found sufficient for the above classes. The important philosophical deductions found possible from the ideas of force and self make it necessary that the sensuous elements of these ideas be subjected to more than ordinary scrutiny. It was in connection with muscular sensations ${ }^{1}$ that these ideas were found to take their rise, and the twofold division of such sensations, into feelings of resistance and feelings of effort, seemed to lend countenance, from the side of the affective consciousness, to such a genesis of these ideas. The validity, therefore, of former positions hangs upon the results of the exposition and discussion of the muscular feelings.

For this reason these feelings have been included both under the head of special sensations and that of common. 
feeling. The analysis of the mass of sensibility which bears the nime muscular feeling will show that this double allotment is just.

Analysis of Muscular Feoling. 1. Kincesthetic Feelings. Suppose for clearness, in the first instance, a case of mechnnical movement. My right arm is lifted swiftly by a frieud, my own attitude being that of entire passivity and non-resistance, and when level with the shoulder, the elbow, wrist, and fingers, are in succession flexed. What do I feel ?

In the first place, I have certain particular feelings from the skin: the feeling of passage through the air, due mainly to a lowering of temperature, and the feeling of stretching where the skin is tightly drawn. The flexing of the finger backwards brings out this feeling of cuticular strain. I also experience sensations of touch if the skin breaks contact or comes into contact with any exterual body, as the clothing of the arm. In the second place, I have certain particular feelings from the muscles, which are clear enough to be easily distinguished: the feeling of contraction in the muscle itself, and feelings of pressure of the parts of the organs against one another, or of a muscle against an obstruction.

Besides these particular and more or less clearly localized feelings, there seems to be a sense of whereness or massive locality of the limb as a whole, in reference to the body. This feeling appears to be made up of elements of tension or strain in the body of the muscle, and of similar strain in the ligaments, tendons, and especially in the joints. In the case supposed, this last feeling is plainly localized in the shoulder and elbow joints.' To these must be added the sensations of muscular fatigue, now demonstrated by Maggiore and

I James makes these joint-sensations of original importance for the notions of direction and space. See his discussion, loc. cit., II. $189 \mathrm{f}$. 
Mosso, ${ }^{1}$ which follow the prolonged use of the same muscles.

Taken together these more or less distinct kinds of feeling are known as feelings of movement. The expression is so ambiguous, however, having been applied by some writer, perhaps, to each of the subordinate feelings in turn, that a better name for the class is at hand in the Greek equivalent kincesthetic feelings. The further point of interest in them is that the nervous process which reports them to consciousness is plainly a sensor or afferent process. So far we may, perhaps, consider our observations above dispute.

This division, so far reached, seems to the writer, after considerable experimental observation, to be justified in consciousness, whatever modifications of it the consideration of the physiology of the muscular sensations may make necessary. There is some doubt as to whether the contraction is felt to be distinct from pressure, and also whether muscular tension is distinct as a feeling from muscular contraction and from the feeling at the joints. For the essential question at issue, however, these points are not important, the main question being whether these sensations are distinct as a class from the class next to be pointed out.

Kinæsthetic Feelings as Immediate or Remote.? The feelings of movement heretofore described have their stimuli in the organ itself which makes the movement. Such feelings are immediate. On the contrary, such movements may themselves serve to stimulate one or other of the special senses, giving a new class of sensa-

' Archiv für Anat. und Phys., Phys. Abth., 1890, pp. 89, 191. Cf. Lombard, Amer. Jour. Psych., III. p. 24.

${ }^{2}$ I here adopt, for the sake of uniformity, the terms used by James to cover this distinction, although I think the words direct and indirect would be more appropriate. 
tions which report the movement. Such movement-reporting sensations from other senses are remote kinesthetic feelings. For example, when I move my arm with my eyes shut and in the presence of noises which prevent my hearing the rustle of my clothing, etc., my sensations of movement are immediate. I now open my eyes and see the arm move and listen closely and hear it: the optical and auditory sensations now added to my consciousness are remote kinæsthetic feelings.

It is important to note that our feelings of movement are perhaps never free from these contributions from remote sources. They almost always enter in a complete statement of the case. And, further, if this be true, we would expect a very close association between immediate and remote feelings of movement: so close that their memories would be as inseparably blended as the sensations themselves. Consequently, whenever we have occasion to estimate the possible effects of such memories as affording or reinforcing stimuli to movement, the presence or absence of such remote feelings must be carefully taken into account.

The nervous arrangement which underlies this confluence of immediate and remote feelings is only another illustration of the dynamic unity of the brain as a whole. The centres for sight and for arm-movements, for example, or those of hearing and of vocal movements, have connecting pathways between them. The activity of one centre stimulates the other directly, and both discharge into the motor course with which one is immediately and the other remotely connected. ${ }^{1}$ On the other hand, instead of reinforcing a discharge, a remote sensation or memory may inhibit it altogether. 'These

1 This is clearly illustrated by cases in which patients are unable to move their limbs as long as their eyes are closed, but can do so when they see the limbs. This means that the direct channel in to the limbcentre is blocked, but the indirect channel through the visual centre is still open. Writers who do not accept sensations of innervation hold that all voluntary movements are stimulated by kinæsthetic feelings either immediate or remote. 
two influences from the same remote centre are illustrated in the fact that in reaching for objects the eye estimates the distance, and leads to our putting forth more effort to stretch across it as the object is further removed; but when a certain distance is reached the same kind of estimation by the eyes leads us to give up the effort altogether. In one case the optical sensations reinforce the stimuli to movement, and in the other they inhibit the movement.

Sequence of the Kinæsthetic Feelings. It follows from the fact that these feelings are afferent that they follow in time upon the actual movement, or upon the elements in it which constitute their stimuli respectively. Unless there be a limb to move, or unless there be sensor courses representing such a limb and sensor centres for the receipt of such stimuli, these feelings could not be experienced.

Furthermore, what is true of sensations in general as regards their possible reproduction or memory is true of these states of the sensibility. The special basis of memory has already been seen to be identical with the nervous conditions of the original experience. It follows, therefore, that the brain-centres which receive and register these kinæsthetic feelings are also the seat of kinæsthetic memories. From the nervous point of view, any form of stimulus which excites the kinæsthetic centre or centres may bring up images of movement, and these images may, under the conditions of intensity, escort, etc., which make illusion possible, be mistaken for real movement; or they may serve to start a brain-process which issues in a series of real movements. What we may call the motor or stimulus value of these sensations is accordingly preserved in a weaker degree in the motor or stimulus value of their memories.

2. Feelings of Innervation. Continuing the analysis of the motor consciousness as it arises from a particu- 
lar muscular reaction, and passing from mechanical to voluntary movement, several more vague and indefinable elements may be pointed out. First, there seems to be a consciousness of the state of the motor apparatus as a whole, as capable or incapable of the movement in question. It is felt in the system as a disposition or indisposition for action. Considered as a state of readiness or the contrary, it may be called feeling of motor potential. It seems to be plain in the different consciousness we have of the right and left arms respectively. Even when fresh from a night's rest, there is a feeling to a right-handed man of greater strength or readiness in the right arm. This is apparently preliminary in consciousness to the actual movement itself.

Fatigue takes on a peculiar character, also, when the fatiguing movement is voluntary: at least, such movement is more fatiguing than mechanical movements. No doubt in the case of voluntary movement more nervous energy is employed. And it seems equally clear that in the two kinds of movement the kinæsthetic feelings remain about the same. If these points are true, we must hold either that all fatigue is nervous, or that there are two kinds of fatigue, muscular and nervous. This last hypothesis is proved by the experiments of Mosso, ${ }^{1}$ and also gathers support from the feeling of intellectual fatigue spoken of above, which would have less of the muscular and more of the nervous element. ${ }^{2}$ As a purely central or nervous feeling, fatigue would fall in the fourth class below.

The two last-mentioned feelings, muscular readiness and fatigue, are closely contrasted in consciousness, and it is possible they have a common nervous basis. The feeling of motor readiness may be simply the consciousness of the

1 Loc. cit.

Mosso proves that both are present after hard intellectual work. Ibid. 
unfatigued centre or organism. But such a common ground of connection would not destroy the distinct character of the two sensations, since we feel ready for action whether we subsequently induce fatigue by action or not: and we feel fatigue after prolonged action whether we felt ready for such action beforehand or not. As far as the element common to both is of consequence, it would tend to suggest a nervous basis of fatigue, since the readiness to act seems to be in a measure independent of the muscular fatigue which is brought on by mechanical movement.

It is in point to call attention here also to what may be called hypothetical feelings of expenditure: ${ }^{1}$ hypothetical, because it has hitherto baffled psychologists to abstract the consciousness of the actual outgoing process which this expression designates from the mass of sensations already mentioned, and more especially from the intellectual feeling of expenditure which seems to enter in the act of attention. But, as will appear later, the same uncertainty attaches to the attempt to separate such intellectual feelings of expenditure from the other motor phenomena which accompany it. If analysis should prove that there are feelings of intellectual expenditure, then we would have in that fact an analogy in favor of feelings of physical expenditure: and the analogy would hold also the other way.

Sequence of the Innervation Feelings. From the nature of each of the so-called innervation feelings, its time of happening may be determined in relation to the actual movement. Feelings of readiness are present with more or less strength in consciousness before the movement takes place; feelings of fatigue seem clearly to follow upon continued muscular strain. Yet if weidentify fatigue with unreadiness for motor activity, it may be said to precede that activity just as readiness does, both

${ }^{1}$ It is to such supposed sensations arising from the outgo of energy in the motor courses that the phrase "invervation feelings" is usually applied. 
being probably central feelings. Feelings of expenditure, again, would lie, to the unreflecting observer, midway between readiness and fatigue, and would accompany the actual discharge of the motor elements into the nerve-courses.

Beaunis analyzes the entire experience of a case of muscular movement into six elements, i.e., sense of amount of force necessary, ${ }^{2}$ of the extent, rapidity, duration, direction of movement, and, finally, of situation. The real nature and physical basis of these feelings must not be assumed under cover of the names given to them by unreflecting observation. It is possible that the above description of consciousness may have to be adapted to the results of physiological experiment and pathological observation. Nothing in consciousness is more deceptive than the order in which its events seem to take place.

In addition to these sensuous feelings, there are certain other modifications of consciousness involved in voluntary movement, such as consent, effort, etc., already spoken of. These are so evidently preceded by an ideal construction that they can properly be estimated only in connection with phenomena of voluntary movement."

IV. Nervous Foelings. Under this heading we have to consider the forms of sensibility shown by the nerves themselves: they are in so far strongly contrasted with the foregoing classes, since in the case of the organic, cutaneous, and muscular feelings, the nerve conducts the sensation from some other organ or part of the body. These feelings may be produced by various artificial experiments with the nerve-courses, though usually-since only his own nerves are capable of reporting the necessary information-the psychologist is shut up to cases of accident or disease for light on the subject, except as far as the treatment of the nerves may be harmless.

In the first place, the nerves are capable of the most acute pain. And nervous pain seems to have a more

'Sensations internes, p. 62.

${ }^{3}$ Chap. XV.

${ }^{2}$ Weber's Kraftsinn. 
positive and, in consequence, more agonizing character than pain from other kinds of tissue. For the present, however, the nature of nervous pain and pleasure may be relegated, as before, to the fuller discussion of the tone of sensibility below.

A variety of feelings arise from a nerve when it is subjected to pressure. If a small band of rubber be stretched around the upper arm, these sensations are brought into consciousness: namely, a tingling in the extremities, the peculiar sense of a limb's being "asleep," and finally numbness; in this order, with increased or prolonged duration of the pressure. These feelings are often accompanied by insensibility of the skin and muscles. The same class of sensations follow from the mechanical stimulus of the nerve-trunks in the stumps of amputated limbs.

Another series of feelings depend upon the condition of the nervous system as a whole. Among them may be mentioned nervous shock, exaltation, and depression. Then there are states of nervous hyperæsthesia, or restlessness, so-called "nervousness." Other conditions bring on feelings of alarm, danger, and anxiety. What can express the alarm a man feels when he awakes and finds the arm he has been lying upon completely dead to all feeling or control? To these massive nervous feelings is to be attributed much that passes for disposition and temperament. A very little trifling with his nerves makes a brave man cowardly, and causes the prudent man to lose his self-control. There are indefinable thrills which pass over the being of the opiumeater, depths of feeling-experience which the victims of exciting drugs alone understand. The foundations of things seem to be upheaving and all landmarks of feeling and knowledge disappear in the whirl of unreality and horrible darkness, when the surgeon's knife grates upon a nerve. 
Further, electrical stimulation of the nerves causes another series of feelings, what we may call electrical feelings: peculiar tingling in the organ, a knocking sensation, or longitudinal feeling of collision, such as the sensation in the elbows when a mild electrical stimulation passes through the arms. Further, electrical stimuli are capable of rapid summation, and give rise to the most excruciating pains.

The analogy between nervous force and electricity is so close, however, that instead of producing a new elass of sensations, electrical stimulation may be applied to the different centres to call forth the peculiar feelings which are normal to these eentres respectively. In this way, many of the feelings already mentioned may be artificially produced.

'The relations of these various elasses of sensuous common feeling to one another will be more apparent after the discussion of the pleasure and pain common to them all.

Physiological Proof of Distinct Common Feelings. That these general divisions of common sensibility have, at least in part, a physiological differentiation is shown by the possibility of destroying certain of them without impairing others. Under progressive anæmia, or loss of blood, the following feelings are lost in the order named-those named subsequently to any particular one remaining intact when that one and those named before it are destroyed-namely: delicacy or coördination of movement, delicacy of touch, pain, voluntary movement, electric feelings, muscular irritability.' Genzmer has found that infants show no painreflexes for some few days after birth.

Whatever other meaning this order of extinction may imply, it indicates functional nervous differenees corresponding to the different sensitions. The view now taken is opposed to the postulation of different kinds of nerve-courses for the different feelings: the law of indifference of function contra-

${ }^{1}$ Richet, loc. cit., p. 138. Cf. also Foster, loc. cit., § 683. 
dicts this. There must, therefore, be differences in the molecular processes induced in the common sensor nerves by the particular stimuli which are reacted upon by the centres in the form of different kinds of feeling. The essential fact of physiological function, therefore, in each case, is the specific conscious quality imparted to a stimulation at the central seat : a quality peculiar in its limitation to the form of stimulation which excites that seat.

On sensation and common feeling, consult: references given under Sensation in Senses and Intellect, p. 115 ; James, loc. cit., vol. II. chap. XVII; Dewey, Psychology, chap. XV and refs. p. 260; Richet, Récherches sur la Sensibilité ; (experimental introduction) Sanford, Amer. Journ. Psych., Iv. pp. 141 ff.; Wundt, Phys. Psych., 3d ed., I. p. 496 ; Maudsley, Phys. of Mind, chap. vI ; Galton, Inquiries into Human Faculties, p. $27 \mathrm{f}$. ; Beaunis, Sensations Internes, chap. I; (contrast) Wundt, Menschen und Thierseele, 13-15te Vorles.; Höffding, Outlines, V. A. and VI. A.; Volkmann, Lebrbuch, \$\$3245 ; Lotze, Medicinische Psychologie, bk. II. cap. 1 and 2 ; Sergi, Psychologie Physiologique, liv. I. chaps. III and IV ; (development of) Preyer, Mind of the Child, part I. chaps. 1-7; Wundt, Theorie der Sinneswahrnehmung, pp. $376 \mathrm{f}$.

On the questions concerning muscular sensations: James, loc. cit., II, pp. $493 \mathrm{ff}$.; Beaunis, Sensations Internes, chaps. VIII to XIV; Bastian and others, Brain, 1887; Münsterberg, Die Willenshandlung; Bain, Emotions and Will, pt. II. chap. II ; Wundt, Phys. Psych., 3d ed., I. pp. 289 ff. and 397 ff., and Mind, 1. pp. 161 ff.; Fouillée, Revue Philosophique, XxvII. pp. 561 f.; Mach, Beiträge zur Analyse der Empfindungen; Lotze, Medicinische Psychologie, in loc.; Harless, Fichte's Zeitschrift fïr Philosophie, Bd. 38 ; Ferrier, Functions of the Brain, 2d ed., pp. 382 f.; Loeb, Pflüger's Archiv, xuıv. p. 1; Féré, Sensation et Mouvement; Mosso, Gesetze der Ermüdung, Archiv fiir Anat. u. Phys., Phys. Abth., 1890, pp. 89 f.; Martius, Philos. Studien, vi. 2 ; Goldschneider, Zeitschrift f. Klin. Medicine, XVI (Abstract Amer. Journ. Psych., II. 514); Müller and Schumann, Pflüger's Archiv, XLv. 37 ff.; Binet, Revue Philosophique, xxviII. 470 ffi.; Féré, Revue Philos., xxvII. 37 ff.; Herzen (nervous fatigue), Archiv des Sciences, Sept. 1887.

Further problems for study: Contrast-phenomena ;

Muscular sensations (devise experiments) ;

Experimental questions regarding sensation. 


\section{CHAPTER V.}

\section{SENsUOUS PLEASURE AND PAIN.}

Is the classification of sensuous feeling above, an important factor under each head was the tone value or accompanying pleasure or pain. This was found so universally present in varying sensuous conditions that it was left for more especial consideration; it is therefore not the universal presence of pleasure and pain as a characteristic of sensibility that concerns us here, but only its character as accompanying and entering into sensuous feeling.

\section{§ 1. Physical Conditions of Pleasure and Pain.'}

General Conditions of Pain. Before an attempt is made to report the more general organic conditions of hedonic tone, the empirical cases of the rise of such pleasure or pain should be enumerated. After that, perhaps, some general characteristics of all such cases may become apparent and serve to throw light upon the wider question.

Phenomena of sensuous pain, which may be considered first, are clearly marked. The determination of the truth of each statement in regard to its rise may be made the subject of an immediate appeal to consciousness.

1. Intensity of stimulation is a cause of pain. The actual experience of such painful intensities in the cases of special sensation leads us to look for it in all forms

${ }^{1}$ For the sake of economy of space, the word tone may be used for the expression "pleasure and pain." 
of sensibility. A blinding light is painful ; a loud noise very close to the ear, rapid friction of the skin, great pressure upon the muscles, etc., all give rise to painful tone. It is true, also, that very strong tastes and decided odors are disagreeable or soon become so : but the case of these sensations seems to differ in some respect from that of the senses which report acnte pain, properly so called. Sensations of temperature, again, either heat or cold, give us positive pain when the degree of either stimulus is very intense. It is possible that the apparent difference between taste and smell and the other sensations, in this respect, may be due to the fact that in them the end-organ seems to have a chemical function, while the other end-organs are simply mechanical. But it is enough here to point out the fact that some tastes and odors are always disagreeable, however slight the stimulation be, and that others seem to be always pleasurable, however intense the stimulation. Bitter tastes, for example, are always normally disagreeable, and sweet tastes normally agreeable.

2. Inflammation. The same painful effects follow ordinary degrees of stimulation when an organ is in an inflamed condition. Irritation is painful when the skin, for example, is stretched or distended. In diseased conditions of the eye the slightest degree of light may be painful.

The same is true also of the nerves themselves. Inflammation may extend to the nervous tissue : it is then sensitive to slight degrees of stimulation, and the reaction is painful. This painful tone is present often under intensities of stimulation to which the nerve is not ordinarily sensitive. The general fact of this paragraph is expressed by saying that a condition of sensor or motor lyyperæsthesia extends also to the painful element in sensibility. It may also be added that the opposite is 
uot always true, but may be: namely, that sensuous anrosthesia extends to the painful element in sénsibility. In other words, tactile or muscular anæsthesia is not always accompanied by analgesia.

3. Summation of Stimuli. A painful reaction may be brought about by the summation of stimuli themselves not painful. Several electric sparks in succession are painful, where one is not. This is probably only a further application of the fact that high intensities are painful. It is given a separate place, however, since here the high intensity does not become so until it reaches the centre, while in cases of intense stimulation the intensity is such at the point of application on the periphery.

4. Appetites or Impulses when denied give rise to pains of want. Such pains are usually periodical, and indicate a lack injurious to the organism.

Less General Conditions. Besides the above, several more special conditions bring about a painful reaction in some one or more of the various divisions of feeling. Exposure to air is a cause of pain to tissue normally protected by the skin; disuse, or too slight stimulation, occasions pain in the more complex of the special senses, as sight; lack of accommodation of the organ to its stimulus has sometimes disagreeable tone, which is exaggerated when the stimulation is intermittent. 'The tone of the organic feelings seems to arise from any obstruction of the organic functions, such as laceration, cramp, repletion, etc. Intermittence of stimulation is also a frequent cause of pain, probably from the failure of the organ to accommodate to the broken stimulus.

Empirical Facts concerning Pain. There are, in addition, certain facts brought out by physiologists which throw light upon pleasure and pain. First may be 
mentioned the intermittence of pain: the greater and less intensity of painful feelings at successive moments, the stimulus remaining constant. It is plainly seen in electrical stimulation-a clear rhythm, or rise and fall, of the painful tone. A headache usually proceeds by throbs, a toothache by jumps, and a felon on the finger changes its feeling from a dull ache to a paroxysm of overpowering severity. That it is due to nervous causes, and indicates the ebb and flow of central processes, is claimed from such phenomena in intermittent fever; but in some cases it evidently depends upon the rhythm of the vascular system, the distension and reaction of the blood ressels.

Another kind of intermittence is brought about by the coming and going of the attention. The effect of the attention in increasing the intensity of affective. states is familiar; hence we would expect that the concentration and withdrawal of the attention would have a marked influence upon the rise and fall of pain. Further, we know that the attention, even when concentrated as steadily as possible, is rhythmical : so here. appears a further possible explanation of the intermittence spoken of. ${ }^{1}$

As to which is ultimate, the rhythm of the nervous system or that of the attention, it is not in point to inquire. No doubt they rise together and influence each other. The very intimate connection between the vivid presence of a painful state under attention and the imagination of the same, comes out in the hypnotic sleep : a mere suggestion of a certain physical pain throws the patient into untold agony as real as any pain could well be; and a counter-suggestion removes it.

Another interesting fact of painful feeling is what is called its irradiation or diffusion. The locality of a

1 On the periodicity of feeling, see Ladd, Physiological Psychology, p. 508, and Bain, Emotions and Will, pp. 42, 43. 
painful stimulus is less circumscribed as the stimulation becomes intense. Besides the intensity, or quantity, feeling takes on a massive or spread-out quality. It is probably due to a real spreading of the cause of the painful feeling over a greater area, both on the periphery and in the central seat.

Again, we may note a delay in the conscious awareness of pain compared with the appearance of the feeling whose tone it is. Even when the stimulation is a very strong one, the feeling is clear in consciousness before any pain is felt. A blow, for example, is felt as contact or pressure a fraction of a second before we begin to suffer from it : a burn is particularly long in reporting itself as pain. This is probably due to the fact that the full force of the stimulus is not reported at once, but that the organ accommodates itself to it by a series of partial transmissions. These transmissions are summated at the centre, and the result is a sufficiently intense central stimulus to occasion a painful reaction. This delay may be measured by comparing the reaction time of a painful stimulus-say the decided prick of a pin-with that of a simple contact sensation at the same point on the skin. ${ }^{\text {' }}$

Further, the duration, or lasting quality, of a painful state of sensibility is remarkable. Pains do not pass away, as painless sensations do, when the stimulation ceases. The recovery of the organism is very slow. What is called an after-image of some sensations seems here to be more truly an after-fact. It is probably due to the fact that the intenser degree of stimulation necessary to pain gives more decided and lasting character to the nervous change it works than feeble stimuli do. This is supported by the observation that pains are more distinctly and easily revivable than other repre-

'See Funke, Hermann's Handbuch der Physiologie, II. 2, pp. 298-300. 
sentations. A painful experience seems to hover constantly around us, and thrust its unwelcome presence into our gayest hours. When we remember that a revived image occupies the seat of the original experience, we only have to assume a more lasting effect to have resulted from a painful sensation, to account for its more easy reproduction.

Finally, pain lowers the temperature of the painful region.

Conditions of Analgesia. Insensibility to pain under conditions usually painful may be brought about by various agencies. Cold of very great intensity has this effect, pain becoming very acute and then subsiding altogether, as the temperature is lowered. The withdrawal of blood from an organ makes it insensible to pain. Lowered sensitiveness to pain, however, is always preceded by exalted sensitiveness, as in the evident case of cold. Apparent absence of pain is experienced when the intensity of a painful stimulus is suddenly lowered, even though the second intensity would be painful under other circumstances.

Pain as Feeling and as Tone. The conditions of pain now pointed out are conditions in the operation of the various modes of sensibility, general or special: that is, we have been observing pain as tone. The important question arises: Is pain always thus dependent on a definite form of sensibility, or is it itself, as a form of sensibility, ever found independent of its presence as tone? There are some facts which lead us to believe that pain has a functional independence, whatever we may say as to its anatomical ${ }^{2}$ or psychological indepen-

\footnotetext{
1 Mantagazza.

I.e., whether there are special nerve-fibres which conduct pain, a point on which experimental results are conflicting. See Ladd's citations, Phys. Psych., pp. 125-127.
} 
dence. For instance, pain may be destroyed without impairing any of the other sensibilities, as in analgesia brought on by chloroform: and in general, under the influence of anæsthetics, pain and memory disappear first and together. On the other hand, other sensations may be destroyed while the painful quality of their stimuli remains. Thus, under pressure, sensations of touch, temperature, and muscular movement may be destroyed while pain remains. So, also, under loss of blood in a member, sensations of touch disappear before pain, and both before temperature, electric feelings, etc., as has been pointed out above. In other words, the various elements of common sensuous feeling may be paralyzed separately.

The inference is that pain has a functional nervous basis in some way different from the basis of the sensuous feelings: but not necessarily that pain as tone is different from pain as feeling. It will not be necessary to hold the latter unless we find it impossible to bring both under some common conception. It is in place below to inquire whether we may arrive at any such single conception of sensuous pain, which will explain all the more important facts mentioned.

Physical Conditions of Pleasure. In the case of sensuous pleasure it is not as easy to point out its physical conditions; but in general we find them opposed to those already indicated as carrying painful tone.

1. Moderate Stimulation is pleasurable. This is readily seen in the exercise of the special sense functions : the eye is pleased with mild colors, and the ear with pure tones. Gentle touch, quiet muscular reaction, moderate tastes, are usually agreeable.

There are striking exceptions, however, to this rule. A great many sensations are always painful; when not giving a painful reaction, the organs involved do not affect consciousness at all. So the organic feelings. Certain tastes and odors, also, are always disagreeable. Further, sensuous pleasure, as depending upon moderate stimuli, seems often to be 
no positive addition to the quality of the sensation in question. Sight, for example, under moderate light, seems to contribute no distinct element of pleasure, apart from the pleasure that accompanies the higher emotions which the object seen calls out. This negative side of sensuous pleasure will be adverted to again.

2. Pleasure arises from the Adjustment of an Organ to its Stimulus. Muscular sensations are pleasurable within the range of easy effort. Stimuli of longer duration, which give time for the full adjustment of the organ, pass from the painful to the pleasurable. Feelings for which we are ready by anticipation are enjoyable. Yet this is also subject to the qualification that perfect adjustment seems in many cases (eye and ear) to have no feeling accompaniment whatever, either of pleasure or pain.

3. Activity is enjoyable. By this is meant function within the limits set by the two conditions already mentioned. If activity is pleasurable, it is the moderate activity of a well-adjusted organ. Yet there seem to be more massive organic conditions of activity which are pleasurable, even when such a general function involves some particular pain. The football-player enjoys his sport, even though he is never free from the pain of bruises or scratches. In such cases, the vigor and energy of the larger organs brought into play seem to overpower the protests of the smaller, and silence their complaints. A pain which would make one wretched if suffered in passive silence is forgotten altogether in the pleasure of diligent employment. This larger activity, however, which brings pleasure, must itself conform to the conditions of moderation and adjustment.

Moreover, these pleasures of activity, such as pleasures of the chase, of sports, of general vigor, are more positive apparently than any other sensuous pleasures. The claim already noticed, that in the absence of pain 
many states are not really pleasurable, but merely neutral as regards tone, does not seem to be well taken in this case. A condition of fresh muscular vigor seems to intrude itself into consciousness of its own force, and we become aware of pleasant occupation with no evident reference to the corresponding state of pain. Indeed, the opposite pleasures which result from a cessation of muscular pain-the so-called pleasures of rest-are something quite distinct from these pleasures of activity.

We seem to have a series of tone values in cases of muscular exercise which is adjusted to the capacities of the system : first, pleasures of activity, then pains of fatigue, then pleasures again of rest. The last are perhaps open to the construction given them by the negative theory; but the first, the feelings of activity, seem to involve no cessation of pain of any kind.

Under this head, also, as including any function, and not simply muscular activity, the pleasures arising from the gratification of the organic appetites and instincts appear to fall. They are functions of periodical exercise, and their normal working involves periodical stimulation. They seem to involve pleasure over and above the prompting of painful appetite : though this again is in dispute. It could hardly be said that all the pleasures of the table are due to the cessation of the pangs of hunger.

Relativity of Sensuous Pleasure and Pain. The fact referred to above, that many physical pleasures are only relief from preceding states of pain, finds place with other similar phenomena, under the law of relativity. First, we may say that the existence of either state may under certain circumstances arise from the cessation of the other. Cases of seeming pleasure, which is explained as absence of pain, have already been mentioned. Similarly, the cessation of an active pleasure 
may give us temporary pain and be the only cause of it. An element of higher emotion, however, generally enters in this case. Again, the intensity of pain or pleasure depends largely upon its contrast with a preceding state. After an unusual trip to the country, the painful toil of city life is all the harder to bear: so, after feasting the eyes upon a dish of luscious fruit, the beggar's plate of herbs is all the more unpalatable. So, also, the associations involved often convert pleasure into pain, and the contrary. A little clever deceit will make us enjoy a dish which before we found unpleasant.'

While the law of relativity undoubtedly holds to a limited degree of sensuous tone, yet its application is much more contracted than in the case of higher pleasure and pain. In almost all the examples given of sensuous relativity, an element of ideal or imaginative feeling enters. The full force of the principle, therefore, can be shown better in a later connection. ${ }^{2}$

\section{§2. Resulting Conception of Sensuous Pleasure and PaIN.}

From the foregoing brief description of the conditions under which sensuous tone arises, we may put all such feelings under two larger physical categories. A careful examination of these conditions will show that all pleasures and pains involve either a state of change in the organic tissue, in the way of integration or disintegration, or a change in the relation of the organism to its environment, in the way of adjustment or misadjustment. These two aspects of the case may be considered separately.

Pleasure and Pain as resulting from Integration and Disintegration. Considering pain from the side of the

${ }^{1}$ On the relativity of feeling in general, see Dumont, Théorie Scientifique de la Sensibilité, p. 78.

${ }^{2}$ See Chap. X. §I, and Chap. XI. $\$ 4$. 
organism, it is easy to see that all the pains of the body are due to disintegration of tissue, except those cases in which any amount of stimulation seems to result in unpleasant tone, such as tastes always unpleasant. That is, very intense stimuli are known to iujure, tear, wound the organ stimulated: stimuli summated to a painful degree have the same effects. The cases of stimuli which are always painful may be brought under the same category if we find it possible to view the response itself as a sign of such disintegration: a position which the chemistry of tastes and smells at least does not dispute. Bitter tastes, for example, we may well consider as resulting from a stimulus damaging to the taste apparatus: so with strong acids.

Yet we cannot say that all disintegration is painful, for the moderate stimulation which usually gives pleasure is also moderate disintegration. Any stimulation whatever involves expenditure; such expenditure meaus the liberation of energy before stored up, and this using up of energy is work done in the tissues. Hence we are obliged to say that under some conditions, at least, disintegration is pleasurable: so the pleasure of exercise.

On the other hand, integration is sometimes pleasurable, as in the case of pleasures of rest; but integration is sometimes painful, as in the pains of inactivity and disuse. What, then, shall we say?

The state of the case seems to be about this: the life-process is a process both of integration and of disintegration : the organism is built up, but is built up by exercise. Expenditure is the law of acquisition. On the other hand, disintegration may overstep the legitimate expenditure of the life-process; and integration may be too continuous to permit the proper expenditure demanded for the life-process. 
If, now, we consider pleasure and the absence of pain the accompaniments of the normal life-process, and pain the accompaniment of any organic event which interferes with the life-process or checks it, we seem to have a consistent conception; it explains the facts, as far as integration and disintegration are concerned.

Certain instances of pleasurable stimuli which have an ultimate damaging effect are urged in opposition to this theory : for example, deadly poisons are often sweet to the taste; food is relished after hunger is satisfied and when further indulgence is harmful. Such cases admit of ready explanation, however, when we remember that the differentiation of function in the nervous system has resulted in divisions in structure to such an extent that damage or benefit to one organ is not realized at once, or perhaps directly at all, in others. Hence what is pleasant may be beneficial or stimulating to the immediate organ affected, and at the same time prove injurious to the whole, when its influence is sufficiently spread. ${ }^{1}$

Pleasure and Pain resulting from Adjustments and Misadjustments. It has already been made evident that integration as an organic process would not include all the phenomena of pleasant or unpleasant tone. A variety of cases point to the relative adjustment of the organism to its stimulating environment as a principle of perhaps equal importance. Wherever such misadjustment is so overpowering as to affect the tissue of the organ in question, the resulting pain comes clearly under the principle of disintegration; but when such positive effects are not clearly present, the fact of misadjustment is yet sufficient to cause pain. Such is the disagreeable quality of musical discords, glaring colors, unaccustomed muscular movements, etc.

Wherever, therefore, there is conscious feeling at all attaching to the adjustment of a sense organ, we may

${ }^{1}$ Cf. Grant Allen, Physiological Alsthetics, chap. II : the fact was long ago explained by Jessen, Versuch über Psychologie, p. 271. 
say that adjustment is pleasurable and misadjustment painful.

This principle might be expected to follow from and supplement that of integration : the conception of pleasure and pain as factors in organic development would lead to such a twofold view. If the nervous system is to develop in progressive adaptation to a widening environment, and if this progression is to be accomplished by a series of integrations within the system, then, in the forms where consciousness is discovered, some conscious value should attach to states of profit or damage in both members of this couple. Adjustment and integration should be of conscious value, i.e., pleasurable: misadjustment and disintegration should be consciously avoided as harmful, i.e., as painful. So tone becomes the element of conscious life-conservation in both aspects of nervous fanction.

Accordingly we are thrown back again upon the requirements of the life-process. The adjustment element seems subordinate to a degree, from the point of view of tone, to the integration element. Too much integration, for example, becomes painful and compels new adjustments. After being shut up in the house all day I am forced to adjust myself to the storm outside; the pain of denying the demands of the organism overbalances the discomfort of getting wet. But this widening of adjustments then passes from the painful to the pleasurable: my daily walk becomes a pleasure to me even in the worst days of the year. And the same is true when changes in environment tax the vitality of the organism. Pain of misadjustment gradually yields to the adaptive integration of the centres, and we become " acclimated," "acquire a second nature," etc. Of these two factors in pleasure and pain, the integration factor corresponds to the central function of the nervous system, and the adjustment factor to the receiving and reacting function, as this distinction has already been pointed out. 
General Conclusion on Sensuous Pleasure and Pain. It now becomes evident that in the life-process we have the raison d'être of pleasure and pain. But by life-process we must be careful to include life-development as well as simple life. The simple present life of an organism as constant function is more than covered by the facts as we have observed them: pleasure and pain have a prospective future reference as well-reference to a fuller development and potential growth. Accordingly, sensuous pleasure may be defined as the conscious effect of that which makes for the continuance of the bodily life or its advancement; and sensuous pain, the conscious effect of that which makes for the decline of the bodily life or its limitation.

Interpretation of Relativity. The relativity of pleassure and pain, therefore, is a relativity in the long run. Pleasure is the cessation of pain in the sense that a state pleasurable on the whole is a state of better or betteradjusted function by reason of the pains already experienced. So pain is a cessation of pleasure ${ }^{1}$ in the sense that a state on the whole painful is in some respect a state of worse or worse-adjusted function by reason of earlier pleasant states.

But absoluteness must also be true in the sense that single pleasures and pains must be what they are in the present state of the organism. Many painful states are painful independently of the pleasurable states at. present possible, and pleasurable states are pleasurable independently of possible painful states.

The definiteness of pain exceeds that of pleasure, however, as regards both the physical process and the form it takes in consciousness. Painful states have an acuteness or pang that forces itself upon us: while pleasures are more diffused, general, and unremarked.

${ }^{1}$ So Renouvier. 
Pains, for the same reason, are more easily and strongly reproduced, as appears more forcibly in the case of ideal feeling, considered below.

The further justification of this theory follows from the examination of other and older theories, the claim being that they all have due recognition in this formula. It is remarkable how slightly theorists have differed on the subject after all, in spite of their antagonisms and jealousies, and how easy it appears to throw the different aspects of the case under a broader conception.

\section{§ 3. Theories of Sensuous Pleasure and Pain.}

Among the various theories of the nature of sensuous pleasure and pain, three great classes may be mentioned according to their recognition of one or other or both of the elements pointed out. That is, some theorists make the organic (or mental ') integration factor the essential one, and neglect the adjustment or evolution aspect of the case : this we may call the static or absolute conception. Others go to an extreme in their estimation of the relativity of tone, and of feeling generally ; losing sight of the constant aspect which has its basis in fundamental elements of structure-structure to which adjustments must be held subservient, as has been seen above; this we may call the dynamic or relative conception. ${ }^{2}$ The third theory is more comprehensive, and gives due recognition to both the empirical determinations; it is the theory presented above. Its roots in the history of doctrine will be traced below; and here, as elsewhere, we find the oldest and plainest conception to be most true.

'So the Neo-Kantians and idealists generally, who hold that feeling us given in consciousness is always made absolute in the knowing process : its worth to us is its represented worth to us.

'So Spencer and his followers: see, especially, Dumout, Theoris Scientifique de la $S$ nsibilité. 
This theory results in what may be called the genetic conception of sensuous tone.

The theory which is called above static may be either a physiological or an intellectual theory. The clearest statement and defence of the view which confines integration to the nervous basis is, without doubt, that of Grant Allen. He defines pain as " the subjective concomitant of destructive action or insufficient nutrition in any sentient tissue," ${ }^{1}$ and pleasure as "the subjective concomitant of the normal amount of function in any such tissue." This, it is plain, is an adequate statement of the integration or organic side, but this would still be true of a fixed self-repeating organism ; and if pleasure and pain are to be in any sense the vehicle of progressive adaptations to environment, we must transcend this definition. "Pleasure and pain are not prophetic," as he truly says, as regards the secondary effects of one single stimulus-the poisoning which follows the sweet taste of sugar of lead : but they are prophetic of the effects of future stimulations of the same kind. For example, the pleasures of exercise under incidental pains of misadjustment are prophetic of similar pleasures without pain, when the same muscles have better adjustment in consequence of this exercise.

Physiologically this account, which is that also of Spencer ${ }^{2}$ and Romanes, ${ }^{3}$ may be considered sufficient, provided that degrees of adjustment of the organ to its stimulus, to be felt at all, involve some change in tissue as respects integration. But as conscious states, the feelings of degrees of adjustment are so clearly distinguished from those of organic function itself, that the two conditions become separable. And this we shall find justified further, if it turns ont that there are intellectual or ideal feelings of pleasure and pain distinguishable from such sensuons feelings; and again, if in these ideal feelings we find the same distinction between feelings of function and feelings of adjustment.

The intellectual view of sensuous tone illustrates another aspect of feeling considered as the inner side of function. Hume and Locke have no feelings apart from "secondary impressions" and "ideas." The Neo-Kantians refuse to allow pure feeling, except as "sensuous impulse," and this impulse

1 Physiological Alsthetics, p. 29.

${ }^{2}$ Principles of Psychology, vol. I. part. Ir. chap. Ix : pain arises when activity is either too slight or excessive.

- Mental Evolution in Animals, pp. 105-111. 
is to be utterly discredited and driven out upon the bleak highways of empirical absurdity. A feeling to be folt must be known to be felt: but to bo known to be felt it must be related to a self that knows : to be thus related it must be bronght under the general a priori rules of the self-identical thinking subject, and so on through the mazes of a terminology which makes a sane man shiver ; for where is the warmth of feeling after such a process? It is sufficient to say here, that sensuous tone is much simpler than this. If I put my hand on a red-hot stove, I do not wait for the feeling to be taken up into relation to my rational self-identical subject, subsumed under the general notions of unity and eausality, and schematized in the productive imagination; and then decide that my sensuous impulse is worthy of a movement of my self-identical subject for its self-realization in time! No, what I do is to feel the pain and remove my hand, and it is probable that I do not even know what hurts me till my hand is in my pocket again.

In short, feeling is not knowledge, and we want no theory that tells us that it is. ${ }^{1}$

The Herbartians, again, make feeling dependent on the intellectual function considered as a play of representative elements or forces. The inadequacy of this theory as an account of sensuous feeling is so apparent that thinkers of this school make a sharp distinction between sensation and feeling, ${ }^{2}$ and rule the former out of account altogether. But of course this is tantamount to an acknowledgment that the ideal theory of feeling does not cover the whole of sensibility.

On the other hand, the relative theory finds an able exponent in Dumont." To him the stability of the life-process and its integration are secondary to its adjustments. All adjustment is pleasurable, but adjustment means, in so far, equilibrium of function and conservation of self. Hence all expenditure is painful and all integration pleasurable. So pleasure is an increase in the store of disposable energy, and pain a decrease.

'This position may be met-apart from facts, such as the pleasures of exercise-by one of the points of relativity which Dumont himself urges, i.e., that habit, while it fixes and intensifies knowledge, diminishes feeling. " Now, if pleasuro

1 Leibnitz brings in feeling under cover of his " unconscious presentatlons," and Hegel detines feeling as "obscure knowledge."

2 Nahlowsky, Das Gefühlsleben, pp. 17 and 30: also Lindner, Empirische Psychologie, p. 152, Anm. 3.

${ }^{3}$ Loc. cit.

- 1bid., pp. 77, 78. 
increase with novelty and decrease with habit, does not this show that pleasure accompanies increasing expenditure? Certainly a habitual chain of ideas involves less expenditure than a new chain, and yet it gives less pleasure. Dumont overlooks this, and claims that the breaking up of a habit, which involves much expenditure, is painful: and so it is, because the expenditure exceeds the limits of adjustments inside of the integrations brought about by luabit.

Dumont criticises the theory that makes pleasure the concomitant of moderate activity on the ground, that it gives a fixity and typical eharacter to sensuous feelings, which their meaning in the life-development disputes. 'This criticism we follow as far as holding that the so-called "energy theory" must be supplemented by an adjustment factor: but it does not follow that there are no fixed organic integrations at all, necessary to feeling. ${ }^{2}$ Ferrier takes the relative view. "Pleasurable and painful feelings," he says, " may be regarded as the subjective expression of physical harmony or disharmony between the organism and the influenees acting on it." 2

What has been called the genetic conception is illustrated by a number of historical positions, all approximating the truth, but clothing it in ambignous statement. From Plato to Hamilton, the genetic doctrine has been current in as adequate terms as could well be, before the rise of the development hypothesis. Plato defines pleasure as harmonious energy, and pain as disharmonious energy; but harmonious energy to Plato means the natural state of function, the proper realization of normal organization." Hence we find

1 Loc. cit., p. 54.

2 Functions of the Brain, 2d ed., p. 429.

3 The older interpretation of Plato (see Hamilton, Metaphysics, Lect. 43), derived from Aristotle's exposition of Plato, is inadequate, i.e., that Plato held pleasure to be a cessation of pain. Plato distinguishes between higher pleasures and lower, holds that higher or intellectual pleasures are not relative to pains, and among sensuous states distinguishes some pleasures (those of taste and smell) as not preceded by pain. I am indebted for the following note on the subject to the learned Platonist, Prof. S. S. Orris. "Plato says expressly that there are pleasures which have no antecedent pains,... although the pleasures which reach the soul through the body are generally reliefs from pain (Rep., Ix. 584, 6. c.). He teaches that the highest pleasure consists in harmonious energy (Rep., 1x. $586 \mathrm{~d}$, and $587 \mathrm{a}$ ). Of these painless pleasures, some, he says, are concomitant on pure mental exercises

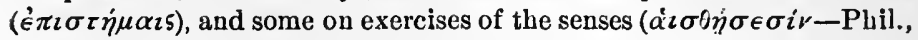


in Plato's doctrine the essential elements of the genetic theory: "energy" seems to mean "function," and "harmonious" seems to mean "well adjusted." 'The fault in Plato, from our present point of view, is lack of confirmation, and lack of adequate realization of the progressiveness of function throngh pain.

In Aristotle the genetic idea reached fuller statement. "The most agreeable sensation," he says, " is the most perfect, and the most perfect is that of the being which is well adjusted to the best of the available stimulations of the sense in question. Pleasure is greatest where sensation is most lively, and where it is exercised upon its most perfect object." : Pain, on the other hand, is lack of "perfection" in either of the two directions; in the mental state itself (function), or in its object (adjustment). Hamilton, to whom belongs the credit of throwing the theory of Aristotle into relief in modern discussion, states the same position in the terms of the faculty psychology, and so gives it a distinctly less philosophical expression than Aristotle's. 'The genetic or development element present in Aristotle's metaphysics of matter and form loses its prominence when translated into the fixed types of the faculty theory. Dumont's criticism " to this effect certainly holds against Hamilton. "Pleasure," says Hamilton, " is a reflex of the spontaneous and unimpeded exertion of a power of whose energy we are conscious," and " pain, a reflex of the overstrained or repressed exertion of such a power."

But ridding these formulas of their confusion of reflexes

66 c). As if differing from Plato, Aristotle says that pain is not in all cases the antecedent of pleasure; that the pleasures of mathematical studies are without pain; and of the pleasures of the senses, those of smell are not accompanicd with pain. But Plato says the same-that. the plensures of learning ( $\tau \dot{\alpha} s \tau \hat{\omega} v \mu \alpha \theta \eta \mu \dot{\alpha} \tau \omega \nu \dot{\eta} \delta o v \alpha \dot{\alpha})$ are unmixed

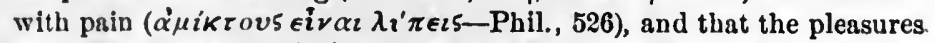
of smell ( $r \dot{\alpha} s \pi \epsilon \rho t \tau \dot{\alpha} \varsigma \dot{\delta} \sigma \mu \dot{\alpha} \zeta)$ are without antecedent pain (Rep., Ix. $584 \mathrm{~b}$ ). . . P Plato says, 'With those who maintain that all pleasures are a. cessation of pain, I do not at all agree '(Phil., 51 a). . . . 'There are pleasures uumixed with pain - the pleasures not of the many, but of the few" (Phil., 52 b)." The element of truth in the Hamiltonian interpretation is that Plato holds sensuous pleasure not to be a good in the ethical sense, and that, in arguing this ethical question, he emphasizes the relativity of pleasure.

1 Free translation from Nic. Eth., x. 4.

Loc. cil., pp. 53-55. 
with consciousnesses, and powers with enorgies, and stating the meaning we get clearly enough from Hamilton's context in this celebrated lecture, ${ }^{1}$ we find no hesitation in saying that he covers the whole case apart from the above criticism. Thus, translated into present-day terms, he may read: "Pleasure is consciousness of spontaneous (functional) and unimpeded (well-adjusted) activity (condition)," and pain the " consciousness of overstrained or repressed (functional) (and ill-adjusted) activity (condition)." 'The theory is here limited, however, to its sensuous application.

Stuart Mill's celebrated criticism of Hamilton ${ }^{2}-$ i.e., that in certain tastes all activity is painful-is sufficiently met by either of two suppositions: either that such tastes are cases of direct disintegration of tissue (as certain acids), in which case it is the disintegration and not the taste which is really painful; or, second, that such tastes (rhubarb, Mill's example) are cases where the adjustment is ahead of the integration, and the organism repels the damaging stimulation. It illustrates the criticism quoted above from Dumont, that the theory as held by Hamilton is not relative enough, and marks the necessity of substituting the word "condition" for activity or "power," in the Hamiltonian formula.

Other theories which we may still include under the genetic idea are much less adequate. They emphasize some one aspect, to the concealment of others. Descartes makes pleasure and pain a "consciousness of greater or less perfection." True, as far as it goes, if by cousciousness we do not run into the absoluteness of a conscious judgment of such perfection." Spinoza is truer in saying "pleasure is the pas sage of a man from less to greater perfection," and "pain is the passage of a man from greater to less perfection;" for it brings out the relative side in the word "passage," and bears an interpretation-from analogy of other passages of the Ethics-of "perfection" in terms of full or complete function. His relativity finds its limits, also, in the principle of self-con;servation (conatus), which is the essence of the active thing.

In Kant we find a genetic statement which is, however, not free from the "absolute" rational influence of Wolfe and Descartes. He defines sensuous pleasure (Vergnügen) as the

1 Metaphysics, Lect. 42. Bouillier follows Hamilton.

2 Exam. of Hamilton, chap. xxv.

${ }^{3}$ Descartes seems to have done more justice to the adjustment side in an earlier view of pleasure as "correspondence of the object with the senses ": De la Physique, ch. Iv, quoted by Dumont, loc. cit., p. 45.

${ }^{4}$ Ethics, definitions 2 and 3 at end of book III. 
feeling of the advancement (Beförderung), and pain (Schmerz) tho feeling of a hindering, of the life-process: ${ }^{1}$ yet, as Wundt points out, this advancement or hindrance was in Kant arlived at by a process of unconscious judgment. Lotze and Wundt followed with the distinct claim of such an unconscious intellectual element in feeling, but later retracted it in fivor of a view very near that of Hamilton." Lotze finds a special nervous process involved in pleasure and pain.

Bain recognizes the twofold uature of the facts and calls in a principle of "stimulation" to supplement the general law that "states of pleasure are connected with an increase and states of pain with an abatement of some or all of the vital functions." " He expresses the principle of stimulation thus: "Certain modes of exciting the nerves, irrespective of the rise of physical vigor, . . . are accompanied with pleasure, and certain other moaes with pain." "The meaning of this twofoldness in principle, from the development point of view, is not grasped by Bain. Yet we have ourselves intimated, as he claims, that " it is better, in the present state of our knowledge, not to push either principle to exclusive predominance."

\section{§4. Sensuous Pleasure and Pain as Worth.}

It is important to hold clearly to the fact that the foregoing definitions deal only with our objective knowledge of the physical process. We can only say from introspection that we feel a sensation, or a pleasure, or a pain, not that we feel perfect activity, or advanced function, or favorable adjustment, or self-realization. These are our theoretical interpretations, and have absolutely no part in consciousness. The sensation itself does not carry its own worth as an object of pursuit. What I feel at first, however it may be later identified with my idea about it, is only the pleasurable or painful modifi-

1 Anthropologie, p. 144.

Cf. Wundt, Phys. Pyych., 2d ed., 1. pp. 495-6.

Senses and Intellect, p. 288.

4 Ibid., p. 300.

'Dömrich and Hagen hold positions similar to Bain's. Paffe gives an able statemeut of the genctic view, Considérations sur la Sensibilité. 
eation of my sensibility. Here Wundt warns us by the best of warnings-his own retraction from an intellectual theory of feeling. ${ }^{1}$ Lotze truly says of the theories that make the nervous system the means of a unit expression to consciousness of the activities of the world, that "those speculative meanings are not in the sensation itself; . . . they are all no more than fancies about sensation, not the peculiar fancy of sensation itself:" ? and it is as true of the more sober doctrine of the evolutionists as of the involved conception of the Hegelians.

Pleasure, therefore, is at first pursued not because it is worth pursuit, but because it is pleasurable: and pain avoided because it is painful, not because it is damaging. ${ }^{3}$

On pleasure and pain, consult : Bain, Senses and Intellect, pp. $291 \mathrm{f}$, and Emotions and Will, chap. I. $\$ \$ 8-13$; Grant Allen, Physiolog. EEsthetics, chap. II ; Spencer, Principles of Psych., part II. chap. IX, and Data of Ethics, chaps. II-IV ; Ward, loc. cit. ; (theories of) Sidgwick, Methods of Ethics, pp. 178-94; Romanes, Mental Evolution in Animals, chap. vIII ; James Mill, Phenomena of the Human Mind, chaps. XVII-XXI ; the psychologies, in locis; Beaunis, Sensations internes, chaps. XVII-XxIII ; Wundt, Phys. Psych., I. cap. 10; Kant, Anthropologie, \$§ 58-64; Rabier, Psychologie, chap. XXXv; Bouillier, Plaisir et Douleur ; Dumont, Théorie de la Sensibilité, pp. 125 ff. ; Hamilton, Metaphysics, Lects. 42-44; Bradley, Mind, XIII ; Eth. Studies, pp. 78-144; Martineau, Types of Eth. Theory, II. p. 297 ; Sully, Pessimism, chap. XI ; Grote, Psych. de la Sensibilité ; Richet, l'Homme et l'Intelligence, I; Schneider, Freud und Leid; Kröner, Das Gefühl, Absch. III. See also the references in the text of the present chapter.

'Loc. cit., p. 497.

2 Microcosmus, Eng. trans., bk. I. p. 566.

${ }^{3}$ Further questions, such as the duration of sensuous tone, its relation to attention, the possible indifference of lower sensibility, are more profitably taken up in the discussion of ideal pleasure and pain, Chap. XI. 


\section{IDEAL FEELING.}

\section{CHAPTER VI.}

\section{NATURE AND DIVISIONS OF IDEAL FEELING.}

Ideal vs. Sensuous Feeling. From a study of general consciousness in connection with the nervous system an understanding has already been arrived at as to the nature of sensibility. The term denotes the subjective aspect of consciousness anywhere and everywhere. And this fact of feeling has been inquired into as far as it appears in connection with bodily states, i.e., as sensuous. In considering sensations, however, as special forms of sensuous feeling, we found it possible to draw a sharp line only theoretically between its affective and presentative aspects. Sensations, therefore, mark the transition from consciousness as pure feeling to consciousness as knowledge, as presentation, as representation, as thought,-or best, because most general, as apperception.

The further question, then, is : Is there an inner, or feeling, side to the world of ideas? Are we sensible of, or do we feel, the phases of the apperceptive process? The simple answer of consciousness is, yes; and there is opened before us the great class of feelings called ideal. Ideal feelings, therefore, are the modifications of sensibility which accompany the exercise of the apperceptive function. 
Ideal feeling is then, as Hodgson ${ }^{1}$ says, a new kind of sensibility; it is sensibility accompanying a new kind of nervous process. The apperceptive function has its organic basis in some kind of a brain-process which represents the combining of special centres in the hemispheres and the dynamic union of their energies. If the function performed by the attention is new, so also is the awareness of it, and the modes of mental excitement which attach to its different phases.

Ideal Feelings as Special and Common. The analogy of sensuous feeling serves us to indicate another distinction. Besides certain special feelings-sensationswhich are brought about by the exercise of particular organic functions, we found a great fund of common sensibility-organic feeling-which seemed to belong to the living being as an organism. The motor feelings were found everywhere, the muscles being the most general outlet for the nervous process which brings feeling about. So upon an examination of the "feelings of ideas," we are able to make an analogous distinction. On the one side there are the special kinds of mental excitement, which are developed in connection with particular synthetic processes: memory yields regret, remorse, pride; imagination throws us into expectation, hope, fear, love. Such states of sensibility we may call emotions. They are the special forms of ideal feeling just as sensations are special forms of sensuous feeling. But they do not exhaust the subjective element of this stage of consciousness. There is an undertone of feeling, a basis of sensibility, which is not disturbed during the mutations of the emotional life-feelings upon which all the emotions depend, feelings due to the fact of mental synthesis itself: such are the feeling of reality,

${ }^{1}$ Theory of Practice, 1. pp. 107-8. 
feeling of interest, etc. These we may call common ideal foelings.

Ideal Pleasure and Pain. Further, here as elsewhere, indifference would seem to be impossible: all ideal sensibility would be expected to have tone, as pleasurable or painful. And, further, the general law of apperceptive revival would lead us to expect the picturing of past pleasures and pains to play an important rôle in the development of the mental life.

It will be profitable, accordingly, to turn attention to common ideal feeling, special ideal feelings or emotions, and ideal pleasure and pain, in the order in which they are here named. 
COMMON IDEAL FEELING.

\section{CHAPTER VII.}

\section{INTEREST, REALITY, AND BELIEF.}

General Character of Common Ideal Feeling. The enumeration of the elements of feeling common to the intellectual processes throughout is necessarily partial and inexact. If this were not so, if these elements of feeling stood out clearly in relief in consciousness, they would be special feelings and not common. It is only as we catch an affective undertone and learn the broader phases of our life of thought, that we come to reach such distinctions as that between interest and belief, between belief and effort, and see that these undertones are always present when we think or strive at all.

Accordingly, the names given to these so-called common feelings often overlap in their meanings and are often confused. The general terms of ordinary usage are accepted below, and we shall find that the more special the concept grows under each head-as hesitation, perplexity, etc., under belief-the more definite is the mental movement which it accompanies. The following aspects of common ideal feeling may, on this understanding, be profitably considered : Interest, Reality-feeling, and Belief. ${ }^{1}$

1 The feeling of consent or effort would naturally suggest itself also here as being one of the broadest aspects of intellectual feeling; but it comes up more properly under the detailed treatment of Will below. The feeling of self also can not be adequately treated here, since it is so closely connected with the voluntary life ; yet as a matter of classification it must not be omitted from common ideal feeling. 


\section{$\S 1$. INTEREST.}

A general characterization of interest as a psychological state is best reached when we ask why it is that we act voluntarily in this way or that. The answer must invariably be, because we are interested in this course of action or that. As will appear later, the most important thing about interest is its quality as stimulating the will. A thing is interesting to me when, for any reason, it appeals to my attention-when it is worth looking at - when it is so related to me that I am led to investigate it; and the feeling of interest is this need of looking, investigating, finding out about. A child is said to show no interest when he is entirely satisfied, saturated, with his toy, and leares it.

Now, can we get any clearer idea of the mental antecedents and conditions of the feeling of interest, or must we be content with such a general description? As to what interest is, certainly we can add nothing to the feeling of it in consciousness, just as all feeling is an ultimate subjective fact. But if it be true that feeling is the inner accompaniment of a brain-process, and perhaps of an intellectual process, then our description may be supplemented by the determination of what these added conditions are.

Another use of the word interest-usually in the plural, interests - must be pointed out: a person's interest or interests may mean his advantage. A man is not always interested in pursuing his best interests, as will appear ; the two meanings are not altogether remote from each other, and popular usage of the word in the two meanings has its philosophical justification.

Physiological Basis of Interest and Indifference. On an earlier page, when gathering up our conception of nerrous function, we found reason to recognize two great laws, i.e., the laws of habit and accommodation. And 
occasion was taken to say of habit, that " psychologically it means loss of oversight, diffusion of attention, subsiding consciousness ;" and of accommodation, that "psychologically it means reviving consciousness, concentration of attention, voluntary control - the mental state which has its most general expression in what we know as interest." "In habit and interest we find the psychological poles corresponding to the lowest and the highest in the activities of the nervous system."

Interest, then, is the most general awareness of the process of our intellectual life, and as such represents the highest and most unstable form of nervous integration. Wherever there is the nervous basis of attention and will, there is sufficient physical reason for the feeling of interest. And wherever, by reason of fatigue or disease, attention and will are not called out, the physical process is accompanied by the feeling of indifference; that is, there is then a reversion to a stratum of nervous structure and function which is dominated more by habit.

Physiological research, however, has little to say in detail about the organic conditions of this class of feelings. So indefinite is our knowledge of the central brain-processes, that even the coarser sensuous feelings can not be given any adequate explanation ; and it may be said once for all that the subtler phases of sensibility covered by the term "ideal" fail of all particular characterization from the physical side. Emotional expression in the case of the more boisterous feelings is our only clue-some say an all-sufficient clue-to a psycho-physical conception. It remains, therefore, to inquire into the mental conditions of interest.

Intellectual Conditions of Interest. The general physiological analogies mentioned above lead to several presumptions which we find neatly confirmed by the psychology of interest.

1. Any reaction of consciousness which is repeated without variation becomes uninteresting; the nervous 
process passes from the stage of fresh accommodation to the stage of habit by the process of downward growth.

On the psychological side we may call this the principle of repetition, and say that intellectual repetition diminishes interest. We have only to understand a thing thoroughly to lose our immediate interest in it. Very few novels are worth reading a second time if interest is the measure of worth. It is hard to get up interest. in the departments of study which deal with descriptive details and statements of fact, and present no new openings for thought. "The conversation of our maiden aunts, detailing the illnesses and recoveries of our early childhood, no longer arouse our enthusiasm.

2. On the contrary, new relations are interesting; the nervous growth is "upward," involving higher integratious. Illustrations are not needed for any one who has ever reflected on the passion for news, the course of rumor, and the delights of gossip for all mankind. This may be called the principle of novelty, and we may say that the intellectually new is interesting.

3. The contradictory of the feeling of interest is not indifference, but ennui, mental fatigue, boredom. Indifference means the reign of nervous habit, the draining off of energy in an accustomed channel. But ennui meaus the distaste that arises from exhaustion of energy. It is a positive feeling as truly as is fatigue.

Interest of Discrimination or Exploration.' These intellectual conditions may be set apart as contributing to interest of a particular sort,- the feeling of curiosity, of exploration. It is never realized in its purity because

'It would be interesting to inquire whether all cases of intellectual interest might not be considered modifications of a fundamental interest in recognition. The absolutely novel is not usually interesting : and what a glow of interest arises when what seems new is, after all, recognized as related to earlier thought! 
emotional and other factors mentioned below come to modify the exploring impulse. But in a cold, calculating individual, who looks ahead and weighs the chances, these conditions are most marked. In early child-life, interest is almost altogether of the exploring kind. First, it is physical exploration: the infant explores his own body, then foreign bodies, his room, then adjacent rooms. The direction of his attention is largely accidental, depending upon casual stimulations. Then there begins a kind of moral exploration, the understanding of his own dress, toys, utensils, the fitting of things together, the meaning of facial and vocal expression. The exploring instinct satisfied, his interest is at an end.

This class of interesting experiences, however, belongs to the more superficial, shifting, and variable side of one's life. They represent the come-and-go of the attention as we follow its quick responses. Purely intellectual interest is, therefore, temporary: it does not attach itself firmly enough to its object to cause the latter to become one of our interests or goods. I am interested in the morning paper, the street sights, my afternoon drive, and the debating society; but to-morrow a set of new engagements carries my interest, and the experiences of yesterday, now past, only furnish one or two points at which my permanent life-interests have been touched. What, then, constitutes more permanent interest, over and above the simple interest of the intellectual act of discrimination?

Fmotional and Active Interest. So far interest simply represents a tendency to know. Its objects are mere objects that come and go indifferently to us: when we have learned what they are and how they act, our curiosity is satisfied. But bring them within the line of our emotional or volitional reactions and everything is changed. Does their being what they are or doing what 
they do hare any effect upon me? That is the vital question. The errand-boy in an office carries fifty letters a day to his employer, and they have no interest for him ; he knows them to be letters for X. Y. Z., and his curiosity is satisfied. But let one letter come to himself, and then not the words it contains or the love it brings interests him alone; but the envelope, its sides and corners, the stamp, the address, the very odor of it, fairly burn him with their interesting aspects. Anything, in short, gets interesting which has, besides its relation to other things and people, a power to make me feel and act. I may know the presence of a thing and not be interested; but I cannot feel its presence, and much less can I act upon its presence, without coming to think it to be worth my close attention. And such emotional interest seems to arise in different circumstances, as follows :

1. Whatever directly causes me pleasure or pain excites interest. Here the reference to self is so immediate that the knowing function which the attention brings with it is simply a self-preserving function. I am interested in pain to discover its cause and remove it, and in pleasure to understand and continue it. This is what pleasure and pain are for, to warn and advise; and to say they interest us is only to say that they carry this function into the life of thought.

The feeling of interest, therefore, seems to be an added thing to the pleasure and pain tone. It arises in connection with the apprehending of the tone and its causes. We would hardly say that an oyster is interested when a sharp instrument is thrust painfully between his shells. The intrusion affects him, and it is in his interest to avoid it; but it is truer to say that it hurts than that it interests him. Circumstances can be conceived in which pleasure and pain would lack interest; as, for example, the pain of an incurable physical trouble or a preying mental anxiety. Such pains are 
understood and endured without any but the negative interest of the endeavor to forget them.

This truth applies to all emotional states, with their pleasure or pain tone, as well as to sensational states. Emotion always terminates on an object, and the interest excited is interest in this object : that is, the interest is of an intellectual kind, but exists because of the relation of the object to some outgoing impulse. The objects may differ and have place in different categories according to the quality of the emotions which excite the interest : thus we have such expressions as "æsthetic interest," " moral interest," "sympathetic interest," etc. But interest is not many ; it is one. The variety is in the emotional qualities and in the objects of thought.

2. Equally original is the interest aroused by our volitional life. Ordinarily we act in reference to a thing because we are interested in it, which means because we are impelled by intellectual or emotional interest. But it is still true that, after acting, our interest is greater than before. Any effort expended on a thing makes it more worthful to us. The reader may have only the interest of courtesy in a new method of shuffling cards or of holding his pen; but after one effort, his growing interest will lead him to new endeavors. Again, even when there is at first no thought of a thing, tool, utensil, etc., and it is used only as a means to a more distant end, interest will gather around it for itself after long use. Who does not part, with an interest which is positive pain, from an old pair of shoes or his last summer's straw hat? The increase which accrues to interest by sharing it also illustrates this volitional and emotional element. Sharing is the result of the emotion of sympathy and proceeds by action.

Here, again, it may be remarked that the interest attaches to the object, not to the activity, except in early child-life, when movements are themselves objects of interest. But it attaches to the object because it is re- 
lated to $m y$ activity. No one's else exertion arouses my interest in the same way.

Interest of Custom or Habit. Very slight self-observation is sufficient to show that while repetition diminishes the temporary intellectual interest spoken of, it is still often through habituation that real interests are formed. There is a distinct line beyond which the customary ceases to be tiring and becomes interesting. Before this line of experience, things are faded and washed out; but as we grow accustomed to them, we begin to find ourselves expecting to find them, relying upon them, appealing to them with an interest born simply of old acquaintanceship.

It is undoubtedly through this principle of custom that some of our deepest life-interests are generated. We grow to think of ourselves with certain accessories which have always accompanied us. So a business man's interests narrow down to his business, because all his habits bear upon it. A man of college culture loses his interest in literature and science because his regular routine in after-life does not include such subjects. We become interested in certain classes of people because we are thrown with them. The cure of unfortunate love is separation, and the hope of an unsuccessful suitor lies in the art of keeping himself and his proposals in the mind of the woman he hopes to win.

Transfer of Interest by Association. Among the facts now cited are many which illustrate what we may call acquired interest. Apart from the simple exploring impulse, which works by suggestion, all interest in objects is thus acquired through their association with ourselves or with things already interesting. ${ }^{1}$ The revival of

' This is the obverse statement of the law of association by interest or "preference" (Senses and Intellect, chap. xr. \$2). The two aspects of 
an idea by association serves for the recurrence of the emotional state connected with it, and it is true, as Hume held, that the intellectual link of association may fall away while its peculiar emotional state remains to color the suggesting idea. A dangerous-looking knife is interesting simply from its murderous possibilities. When we question such an emotion closely, we know that it arises from association; but yet the feeling, the interest, comes without definite associative links.

Definition of Interest. A thread of common value may now be detected running through the complex phenomena of interest. Objects are interesting only as they affect us or are associated with objects that affect us. And by the phrase "affect us," we mean-work some change in the sensibility, which tends, by the law of motor-reaction, to realize itself in activity. Given such a modification of the affective consciousness, and interest invariably arises.

Now, such affective modifications may come in two ways. The two great stimuli to activity are pleasure and pain on the one hand, and suggestion on the other. ${ }^{2}$ Suggestion is passing, shifting, temporary : the interest it arouses is intellectual, temporary interest. But pleasure and pain, in all their range, represent the constitutional and permanent. As stimuli to movement, they are recurrent. And the interests they arouse are the deep-seated life-interests already examined. The ordinary distinction between interest and interests is accordingly just.

The common element, further, is an impulsive element

the case may be put thus: When two ideas are repeatedly associated, interest in oue passes over to the other, and when an idea excites interest those ideas will get associated with it which are suited to share or gratify the interest.

${ }^{1}$ See below, Chap. XIII. § 3. 
-a tendency element-realizing its object through the attention, which is the velicle of apperception. Accordingly, in view of all that has been said, we may define interest as the impulse to attend. And since it is in the attentiou that all mental synthesis takes place, we may say, as an alternative statement, that interest is the consciousness of a tendency to think. The amount of interest an object or topic will have for us at any time is the amount of calling-out force it exerts upon the attention, both by direct suggestion and by association.

Interest as Ideal Emotion. Consideration, therefore, justifies the view that interest is the subjective side of the apperceptive function. Habit diminishes interest because it diminishes the intensity and energy of presentative construction; but habit begets interest because it makes deep and strong the lines of associative or representative construction. By repetition, simple suggestions lose their force; but by repetition the moving principles of our nature gain force as stimuli to the relating process of attention.

The place of interest in the mental life has remained anomalous ; it has had no adequate discussion from psychologists. It has been treated, on the one hand, as a stimulus to thought, itself arising independently of thought; and, on the other hand, as the result of thought or attention. We are in a position to see that both are trie. If the simple movements of ittention under the lead of suggestion arouse interest, then it must be called an accompaniment or result of attention. But if it is through interest that affective modifications get an outlet through the attention, then interest, before only exploring, becomes an essential stimulus to voluntary thought. In general, involuntary attention follows the lead of interest of exploration, and voluntary attention requires the stimulus of emotional and active interest.

But heretofore theories of interest have not been well developed. The Herbartians reduce it, like all feeling, to a consciousness of relitions among ideas. Volkmann defines it "as the "relation" of an idea to the group of ideas which

' Lehrbuch d. Psychologie, 3d ed., II. p. 203. 
represents the ego.... Everything interests me of which I can say: I am in it (ich bin dabei)." But this clearly omits the simple interest of exploration. Steinthal ${ }^{2}$ agrees with Volkmann, saying interest is the "readiness of a group of ideas to. assimilate a new idea." Ulrici and Fortlage emphasize the impulse side of interest. Sully has good, but somewhat loose, descriptive remarks on interest. ${ }^{2}$ The Hegelian view is stated concisely by George. ${ }^{8}$

Interests vs. Affects. By affects are meant all stimuli to involuntary attention: " by interests, on the other hand, we mean all stimuli to voluntary attention. The passage from affects to interests is the passage from the passive and reactive to the voluntary consciousnessthe passage from consciousness which has no object. before it, as such, to the consciousness which has such an object. In interests, therefore, we have a step in mental growth of enormous significance in psychological theory. Affects draw the attention mechanically. As far as they are concerned, we have ground only for an effect theory of the mental principle. Is the case different with interests? Have we any new and different. factors in the problem when an object is pictured, found interesting, and intentionally pursued? These questions it is our business to answer later: the fact here must. suffice that interest attaches to something presented, and leads to what we call volition to pursue it.

\section{§2. Reality-FeELiNG.}

The fundamental question of philosophy is: What is real? Is there reality anywhere? Psychology has to do with this question only as far as to determine what we

\footnotetext{
1 Psychologie u. Sprachwissenschaft, p. 330.

${ }^{2}$ Outlines, pp. 83-87. For educational applications see the works on Pedagogics, Appendix A, below.

${ }^{3}$ Lehrbuch d. Psychologie, p. 544 ff.

- Below, Chap. XIII. § 3.
} 
mean by the sense of reality. Admitting a distinction in consciousness between ideas to which we attribute reality and those to which we do not, we note the different subjective effects which the two sorts of ideas have on us: the presence of one sort is the feeling of reality; of the other, that of unreality.

Distinction between Belief and Sense of Reality. Without entering at this point into the grounds of the distinction, two different sorts of feeling may be denoted by the terms reality-feeling and belief. The phrase realityfeeling denotes the fundamental modification of consciousness which attaches to the presentative side of sensational states-the feeling which means, as the child afterwards learns, that an object is really there. By the word belief, on the other hand, we may denote the feeling which attaches to what may be a secondary or representative state of mind, and indicates the amount of assurance we have at the time that an object is there. The idea which has the reality-feeling may be said to have its own guarantee of its reality; it is a given, and my feeling of it is direct acquaintance with it. But the idea to which belief attaches is guaranteed by some other mental state, by what I know about it, or by its connection with ideas already guaranteed. This distinction and its bearings will become clear as the exposition proceeds.

To the mind of the writer this distinction is a fundamental and vital one. Yet it has, as far as he knows, been made nowhere in psychological literature. Its successful establishment is, of course, the task of the following pages. Beginning with the earliest reality-feeling in infancy, we shall endeavor to show how it becomes the complex, and to the psychologist refractory, thing known as belief.

Rise of Reality-feeling. The dawning consciousness of a child-passive consciousness, as it has already been 
called-is filled with affective sensational happenings. All it has at first is feeling, and feeling of one kind. This feeling has no meaning whatever of any kind; for by meaning we mean interpretation in terms of something else, and there is nothing else. Hence it is not the "taking of a representation for an object," as Rabier says." The flash of light, the muscular sensation, the pain, each is simply this, an experience. There can be no distinction corresponding to reality and unreality, inner and outer, subject and object, presentation and representation.

Reality-feeling, therefore, at this early stage, is simply the fact of feeling; nothing more, but this much. Existence is simply presence; but presence is existence, and whatever is, in consciousness, is real.

James expresses this truth as follows: "The candle (first sensation) is its (the infant's) all, its absolute. Its entire faculty of attention is absorbed by it. It is, it is that; it is there; no other possible candle or quality of this candle, no other possible place or possible object in the place,-no alternative, in short, suggests itself as even conceivable; so how can the mind help believing [feeling] the candle real ? The supposition that it might possibly not do so is under the supposed conditions unintelligible." ${ }^{2}$

Now this feeling of presence or reality is not belief; and we have put "feeling" in brackets above, after "believing," ; since Prof. James overlooks the distinction. He is guilty of the "psychologist's fallacy" in saying "any object which remains uncontradicted is ipso facto posited [or affirmed] as reality." " Positing or affirming must carry a distinction in consciousness between the true and the untrue. If this feeling of "presence" is belief, then some other name should be found for the complex thing which we go on to describe

1 Psychologie, 3d ed., p. 267.

2 Principles of Psych., Ir. 288.

s Loc. cit., Ir. 289 and 319. 
in the pages that follow. In dreams the simple realityfeeling is present without belief, as is proved by the fact that the grossest inconsistencies are accepted. This simply shows that consciousness has lost its questioning attitude altogether -belief as such does not arise: but reality is there in its full strength.'

Rise of Unreality-feeling. Further, the early consciousness soon experiences something quite different from this feeling of presence. As soon as appetite and impulse assert themselves, they are felt; indeed they make the keenest demands upon the early sensibility. As we adults look at it, it is a feeling of lack, want, need; but to the infant it is simply a feeling, and a new one. But this new feeling must very quickly get connected with the reality- or presence-feeling: say the sensation of the white surface and warm touch of the milk-bottle, as following upon the lack of food. In other words, a simple presence-feeling becomes connected with a simple absence-feeling. As a matter of fact, the two come together, and it is perhaps the earliest felt distinction in the infant consciousness,-vague hunger-feeling, presence-feeling of taste and touch, absence-feeling when the supply is cut off. This absence-feeling is the first and original unreality-feeling.

Closer examination again shows us that this unreality-feeling has nothing to do with a negation of belief; with doubt or hesitation, the true negation of belief. If the sense of unreality arose as a contradiction of the sense of reality, there would be some justification for this view. But in that case we would not have a sense of unreality, but a sense of the reality of a new and contradictory experience. For example, the early

'Baln recognizes this state; but in calling it " primitive credulity," as well as in treating it as a form of belief, he fails to note the essentiai element of true belief, i.e., mental assertion, which is absent in this simple reality-feeling. Emotions and Will, pp. 510-515. 
consciousness has a single candle before it-a reality-feeling. Suddenly the candle goes out. Darkness is now a new reality-feeling. A memory of the candle persists and conflicts with the present darkness, and a new feeling arises,-doubt, perplexity - the foundation of belief, as appears below. But the unreality-feeling has an entirely different origin-in our active impulsive nature. It comes before there is any conflict, and lingers after such a conflict, distinct from the feeling to which this conflict gives rise.

Degrees of Reality- and Unreality-feeling. Both of these original forms of feeling must have degrees. Not only to the child is the reality of food more intense and consuming when it is hungry than when it is filled, but to the mature man there are realities and realities. Every one of us has his true reality, his real and eternal as opposed to his unreal and temporal. Even external things sometimes seem to bruise and wound us, so hard and stubborn does their reality become; and again, all the world seems thin, flimsy, and unsubstantial. We believe many a fact of which we fail to get a "realizing sense." Simple conditions of the nervous system derange our sense of reality : and emotional conditions suffice to infuse body into our life-experiences or to render them ghosts of profitless pursuit. Confining ourselves, however, now to the infant's life, we may say that his most vivid realities are those sensational states which satisfy his appetites and needs.

All this class of experiences again confirms the separation of these feelings from real phenomena of belief. Ask an intelligent man at random if he believes that four thousand people perished at the Johnstown flood, and he will say yes; then ask an eye witness of that awful calamity, one who helped stretch out a hundred dead, and how much more his "yes" really means! But both believe the facts of the case fully. One has a feeling of reality which the other quite 
lacks. It is this feeling element of present reality that enters largely into religious faith, and is contrasted with intellectual assent. "The devils believe," but the religious man is to realize his belief in that warmth of emotion which kindles active devotion.

Physiological Basis of the Reality-and Unreality-feelings. The organic basis of these feelings, it is easy to see, is nothing more nor less than the organic basis of consciousness itself. Any sensory process has its feeling of reality element, and any tendency to movement has its unreality-feeling, succeeded by reality-feeling, in the sensory process which satisfies it. Further, this feeling of need must arise from a lack of sufficient stimulation in the sensory seat, which lack is itself a stimulus to the motor-process by which the lack is supplied; the connection between the two processes being fixed by heredity and experience.

Looked at more broadly, here is an organism in a world of environing conditions; a certain sensational process represents its best life among these conditions. When it fails of this normal sensational process, its very lack is a stimulus to a motor-process by which the normal sensational process is re-established. Assuming this normal sensational process, whatever it may turn out to be, let us call it the sensational coefficient. By this phrase is then meant the element of nervous activity which, being present, gives a sensation: over and above the activity which gives a memory-picture or arouses an impulse. The sensational coefficient is the activity which is regularly aroused by a real object.

In this feeling of reality we find the mental "predisposition to illusion " referred to in a previous chapter." If the presence of the sensational coefficient gives "real" coloring to a conscious state, then, whenever this coeffi- 
cient is present, reality is reached. But if, by reason of undue excitability from disease, emotion, expectation, or other internal causes, this coefficient is artificially brought about when no reality corresponds; then illusion results.

Time and Space Reference of Reality-feeling. As soon as memory begins its function, the data are present for the construction of the time-notion; and the three temporal aspects of events, as present, past, and future, give coloring to the reality-feeling. The feeling of recognition $^{1}$ arises when the reconstruction of past experience takes place, whether that reconstruction represent real events or not. That is, recognition is independent of belief in the external reality of what we recognize. The feeling of presence again becomes the feeling of present time filled with events; and the construction of further experience as possible in the future gives the feeling of expectation.

The early quality of massiveness or extensity in sensations ${ }^{2}$ is a feeling which accompanies the earliest construction of the spacial form in experience. It seems, in connection with some sensations, to partake of the three dimensions, i.e., feeling of largeness, roominess, bigness, of sounds, muscular movements, touches, etc. These feelings represent the earliest and most incomplete form of temporal and spacial synthesis; they are limited to actual experience and its promise, and are altogether earlier than the developed ideas of time and space.

Our general outcome so far is, accordingly, this : the feeling of reality is simply consciousness itself; it is most vivid when it accompanies a nervous process having the sensational coefficient. The feeling of unreality arises in connection with appetites and impulses which result from the

1 Senses and Intellect, chap. x. \$1.

2 Ibid., p. 109; also see above, p. 98. 
absence of the sensational coefficient in particular sensory brain-seats. This may be called the first stage in the development of the consciousness of reality.

\section{§ 3. BELIEF.}

The feeling of belief is a feeling which attaches to the representative faculty primarily. It is only when memory and imagination come to bring up rival candidates for our acceptance, that we believe or disbelieve. The foregoing discussion suffices to show that something else must be added to the simple feelings of reality and unreality, as these arise in connection with sensations, to constitute true belief. The question of belief, put most broadly, is this: Why is it that, of two images which come into my consciousness, I discard the one as an imagination, a phantasm, and accept the other as a memory or present fact?

Again it is needless to say that, as a feeling, belief cannot be explained any more than any other feeling; it must be felt; and that further remarks are really upon the physiological and psychical conditions under which this feeling arises.

Doubt Precedes Belief. It was said above that the unreality-feeling comes, in cases of appetite, to oppose the simple reality-feeling of presentation or memory. The reality-feeling doubtless attaches at first to a memory of a candle as to a real candle, and nothing contradicts it. But, with other memories, this reality-feeling is rudely disturbed. The memory of food suggested to an infant by vain sucking at an empty bottle no longer has the reality-feeling. Unreality takes its place. So certain memories get labelled as unreal. And it is the discovery of this possible unreality-the discovery of the possible absence of the sensational coefficient, as the impulsesatisfying thing - that is the beginning of doubt. ${ }^{2}$

‘ Only experience-and that means disappointment-emphasizes 
That this is not theoretical only is proved from the observation of young children. They have implicit confidence in everything at first, but soon a stage is reached of hesitation and doubt. Unaccustomed things have so often brought pain, that the new-the strange face, the unusual expression of a familiar face, a new room, a new plaything-are treated cautiously and with manifest distrust. The question is : Can I trust the new image to satisfy my impulse toward it?

Development of Doubt. As the rise of doubt is due in child-life to the failure of a state to satisfy ; to the absence of the sensational coefficient: so all higher doubt can be traced to like conditions. I doubt an image, a statement, a law, because it does not meet the demands that I have a right to make of it if its claim be true. Just as there is a sensational coefficient, so there is an rsthetic coefficient, a moral, and an intellectual coefficient,- that quality in each of these fields which satisfies the demands of my nature in these directions severally. I doubt that a face can be called beautiful, because my resthetic sense is not satisfied with it. I doubt whether tuberculine cures consumption, because my logical sense is not satisfied with the evidence; and so on everywhere.

There are a great many things in our lives which never pass into the stage of doubt or belief at all; things which remain under the rule of the simple sense of reality. My mother's love, for example, is a thing in which I cannot be said to believe. It was one of the first realities of which I became sensible. My reality-feeling in reference to her has never been disturbed one way or the other, and so it has remained undoubted and unasserted. So it is with the religious truth in which one is reared. It is a shock to the sensibilities to ask the question, Do you believe? for the first time; it suggests the possibility of doubt, and puts us under the necessity of turning simple reality into grounded belief. But of other people than my mother-my books, say; and of other truth than religion-my history lesson, say,-I make certain de-

the difference between the possible and the actual." Höffding, Outlines of Psychology, p. 236. 
mands, and condition what is truly belief upon the way these denands are met.

What the higher demands are which $I$ have a right to make of asthetic, logical, and religious truth, in order to uwad my belief, that remains for later consideration. Here it is enough to show that the law of " satisfaction" is one throughout.

Resolution of Doubt. As doubt arises from the attitude of mind toward a new image, so doubt is resolved by an actual resort to experience, as far as that is possible. In the case of sensible things, we try and see whether the image have the sensational coefficient. If the child has once been fooled by an empty bottle, it doubts the bottle at its next appearance. But its method of testing it is always the same: it tries it. Does it get the needful sensation?-then reality is there; if not, then not. In all kinds of belief there are such tests, as appears more fully below.

Nature of Belief. Now the feeling which follows in every case is a feeling of resolved doubt; it is not the simple feeling of reality which prevailed before the doubt, or of unreality as unsatisfied need. It is a larger, freer, fuller state of mind. It is belief and disbelief, or, better, positive belief and negative belief; for the two are one state of mind. And the opposite of belief is doubt, as has been seen. ${ }^{1}$

One only has to question himself with ordinary care to find the truth of this result. The very word belief brings up suggestions of uncertainty. The mental side of this state cannot be separated from the inheritance of associates which swing down the tide of consciousness to attach themselves to it. As long as I am unaware of the real force of a thing, its sensational, emo-

1 The word belief is hereafter used to cover both belief and dishelief; the latter being equivalent to belief in something which negates Ih:t which is disbelieved. 
tional, or convincing quality, I simply let it pass. There are thousands of things about us, social conventions, redtape enactments, customs of dress and daily habit, which I conform to because they are not worth the trouble of a more serious attitude of mind. But what I believe has its pros and cons ; and however vaguely, still really, I am better satisfied with the pros than with the cons. Now for the first time, therefore, we have belief. And from the foregoing its conditions are more or less plain. Of belief in sensible things, the following remarks may be made.

1. It results from the presence of a sensational coefficient after its felt absence. We say felt absence, for simple absence, followed by presence, means simple unreality followed by reality-feeling, with no belief at all. But when the absence of a coefficient is felt, then its incoming presence leads to that mental affirmation of it which is belief.

2. It results from the satisfaction of an impulse which terminates in a sensational coefficient. By impulse is meant any practical need which brings an image to the sensational test.

3. It is a feeling of confirmation and security over and above the feeling of simple reality. This is what is meant in the discussion below by the will element in belief. It is the distinct feeling of ratification which I myself give to reality by being satisfied with it. I consent to it. Without anticipating details which are not necessary here, sensuous belief, and by implication all belief with it, may be defined as consciousness of the personal indorsement of reality.

Accordingly Brentano ${ }^{1}$ strikes the real point of importance in using the word judgment (Urtheil) to mean belief.

1 Psychologie vom emp. Standpunkte. So also Lipps, as I understand him, who gives as definition of Urtheil, "presentation with the con- 
Judgment is the assertion of the consistency of concepts, or of conception with reality. Belief is the subjective or feeling side of such assertion. Inasmuch, however, as it has seemed necessiry to deny judgment proper to the kinds of synthesis which are not assertive in form $(a=b),{ }^{1}$ I cannot follow Brentano in his use of the word judgment. 'There are many beliefs - in the external world, in memory, etc.-which are not formally assertive, i.o., not judgments.

Reaction of Belief on Reality. This may be called the second stage ${ }^{2}$ in the development of the consciousness of reality: the simple reality-feeling has passed into belief. Belief then becomes the test of reality. We turn back ruthlessly upon all we have accepted, and see whether it will staud the tests of reality at this second stage; whether it is meeting the full demands which our credence makes upon it. Realities to me then become what I believe, and what I believe is what meets the requirement of my life.

Kinds of Belief. Broadening our outlook, we are able to distinguish several aspects or phases of this feeling, which we may call respectively belief in the external world, belief in memory, logical belief, belief in ideals, etc. The general theory already set forth leads us to see that in each case there must be an impulse or tendency to a particular kind of experience, and that the reality of that experience must depend upon its capacity to satisfy the tendency involved. Calling, in each case, this ability to satisfy, the coefficient, we have as many coefficients

sciousness of reality" (of any kind, Wirklichkeit), Grundthatsachen des Seelenlebens, p. $396 \mathrm{ff}$.

'Senses and Intellect, p. 286.

'In the words of Hodgson, " existence (belief) is a second intention, a predication about the phenomena of the subjective aspect. The objective aspect is those phenomena plus the reflection-that they are phenomena." Theory of Practice, r. p. 266. 
of reality as there are fundamental tendencies toward objects. $^{1}$

\section{§ 4. Belief in External Reality.}

Its Coefficient. A few more words may be said about external reality as contrasted with the other kinds of reality in which we believe. The question suggests itself: What, in consciousness, is the sensational coefficient? Granted such a nervous process whenever a real object is present, what mental changes does it work?

We are now able to call upon the determinations already made in regard to the grounds of illusion. ${ }^{2}$ The grounds of illusion must be the marks which give the semblance, the coefficient, of reality. Most generally speaking they are two, first very high intensity ${ }^{3}$ and second uncontrollableness. Whenever a mental state is intense, be it sensation or image, and resists all endeavor of ours to modify or banish it, it carries our belief, it is real, as far as sensational tests are concerned, i.e., as far as the sensational coefficient goes. I may often have grounds for distrusting such a stateother coefficients which I invoke as of more worth to me in deciding the case than the sensational tests; but if I had only the latter, if I were merely a being of sensations and reactions, intense persistent states would always and invariably sum up reality for me.

Of these two elements of the sensational coefficient, the latter is more important and essential. Simple

1 The question of "objectives" generally (i.e., Why is it that mental states have objects?) is not here in discussion. Assuming objectives or presentations, Hodgson's "objective aspect" and Pikler's objectiva, the question we ask is, Why is it that some of them are accepted and believed, and others are not?

Senses and Intellect, chap. xII. 32.

${ }^{3}$ Emphasized by Hume, Treatise, part IIr. sects. vII, vIIr. 
reality-feeling attaches to intense and feeble images alike, provided no impulse arise which fails to find its satisfaction in the feeble ones. But in the element of uncontrollableness we have a confirmation of the impulse origin of all belief. Our impulses, our life-needs, are fixed and permanent, not subject to our will or control: so are their satisfactions, the realities we have reached in our life-experience.

Even seeming contradictions in such sense-reports are not sufficient in themselves to controvert belief. Do I hear a voice when my eyes tell me no one is nearhear it with its coefficient of reality? Then I believe it, and believe my eyesight too. The principle of consistency, of such supreme importance in logical belief, has only a subordinate application here. If I do compare my senses and judge which of them to believe, it is either because one or more of them has not the energy or persistence of others, or because I appeal to higher considerations-coefficients-to help me out. In actual fact we do find a class of sensations which realizes the coefficient most directly and distinctly, and thus becomes the arbiter or referee of sensible reality.

Primacy of Muscular Sensations as Giving External Reality. In an earlier place,' touch-with muscular sensibility - was called the "controlling sense," because questions of reality are referred to it for decision. We now see why this is so. It is through muscular movement that will and impulse and appetite, that all outgoing processes, are realized. If natural satisfactions therefore are the basis of belief in external reality, then the medium of such satisfactions must be the medium also of the sense of reality. And further, motor-reaction is itself an impulsive, original thing, and takes place largely through the stimulus of resistance: conse- 
quently the presence of resistances is itself the gratification of the need of motor-development-perhaps the most general and fundamental sensational need that we have. If we could get satisfactions without muscular sensations, then the latter would not be tests of external reality.

Primary Criterion of External Reality. Consequently it is only what we would expect that sensations of resistance become the primary criterion of all external reality. Anything that resists my will is believed to have present reality. And it is not simply resistance through contact, but, by generalization, resistance in any of the classes of sensation. A stifling smoke resists my will to be rid of it, that is, the physiological effort I make to banish it shows me that I have no control over it. But that this is derived from muscular resistance is seen in the fact that I confirm my belief derived from the smell of smoke by searching the house to find the fire.

It is clear that it is the element of present reality in the external world that sensations of resistance guarantee, not, to a great extent, future reality. The element of persistence in the world without depends rather upon the memorycoefficient, to be next discussed. Persistence comes through the sensational coefficient only as far as resistances have duration, i.e., stand constant in opposition to prolonged effort or in gratification of prolonged appetite. There is also probably an element contributory to the notion of persistence, in the accidental recurrence of old resistances when we do not expect them $;^{1}$ but such accidental anti-associational occurrences also come in the shape of pure imaginations, and the test of present resistance is the final appeal in cases of such unexpected experiences.

To the objection that pure imaginations may have the quality of uncontrollableness (fixed ideas), it may be pointed out that in most cases they cannot stand the test of actual muscular resistance, and that when they are abnormally im-

1 Urged by Stout, Mind, xv, art. Cognition of Physical Reality. 
perative they are considered as realities, i.c., we have the beginning of delusion.

\section{§5. Beidef in MeMrory.}

The Momory-coeffleient. By memory-coefficient is meant the coloring of reality which some images have, as representing former states of consciousness: that by which I distinguish a memory from a dream or a creature of the imagination. In general terms, it is the question of recoguition over again. Belief in memory is the feeling which attaches to images recognized; and as recognition has been seen to rest in the diminished expenditure of attention involved in the reinstatement of an act of apperception, we have here a sufficient statement of the intellectual conditions of the feeling of memory-reality. ${ }^{3}$

As feeling, however, two very distinct forms of realityconsciousness attach to memory: first, what we may call the simple sense of revival or recurrence, and second, the belief that what is thus recognized was itself a real objective experience. I may remember a dream, rocognize it, and believe in it as a real memory, and yet be in doubt as to whether it was a dream or a real occurrence when I first experienced it.

The memory-coefficient of belief attaches properly only to the first of these states: it answers the question, What shall I recognize? The further point of feeling-that which attaches to the answer to the question, Is what I recognize a reality ?-requires further inquiry into the nature of the memory in question. Does the memory recognized include memory of the sensational coefficient? Did I believe it to be a real object when I first experienced it? This question determines whether I shall feel it to be the memory of an objective thing or 
no. So with any other of the higher kinds of realitycoefficients yet to be spoken of. Do I recognize a former image of a beautiful face? Yes: but do I recognize it as a living beautiful face? That depends upon the kind of coefficient, sensational, imaginary, æsthetic, etc., of my earlier view of the face. The dependence of belief in many cases upon the sensational coefficient is well put by Hume in his definition of belief as "a lively idea related to or associated with a present impression (sensation)." ${ }^{\mathrm{He}} \mathrm{Hlso}$ points out that false images may get the sensational coefficient by repetition and emphasis."

A further refinement leads to a distinction between the recognition which has already been described as simple reality-feeling as it attaches to revived experiences, and recognition as a form of belief, i.e., as conscious reasoned recognition. Often one is not satisfied that he recognizes a situation till he passes the associative connections before him consciously. The peculiar illusion of being in a familiar place is simple reality-recognition, which we are unable to make reasonable as belief by supplying the missing elements. But both of these kinds of recognition must be strongly distinguished from the recognition of external reality.

Memory-coefficient Proper. The question then, Why do I recognize anything consciously at all?-has its answer in the memory-coefficient proper, viz., because 1 can reproduce it voluntarily by starting a chain of associations leading up to it. I have control over it in this sense, that it is at my command for reproduction. My past is mine only in as far as I can utilize it in my present. I refresh my memory by rehearsing details, and thus bringing up points which, if simply suggested to me without their earlier connections, I might have failed to recognize. So we reach two kinds of present 
reality : present external reality, guaranteed by its independence of my will, and present memory-reality, guaranteed by subjection to my will.

Moreover, there are further modifications of memories due to volition. We can by attention bring out details of outline, strengthen, intensify, or, by neglecting, practically banish a memory: all of which influences external things stubbornly resist.

Completed Criterion of External Reality. Besides the primary criterion of external reality found in feelings of resistance, a secondary criterion is, therefore, supplied by memory. Of the two kinds of memories, both having the memory-coefficient, those which represent external realities and those which do not, the former are important factors in the development of our idea of the world without. Among the trains of association by which memories may be voluntarily brought up are certain muscular trains themselves accompanied by resistances, and the memories brought up by them also resist. It is only these muscular resisting trains terminating in a resisting experience which carry belief in external things remembered. For example, I remember equally a merman and a salesman. I can get the shopman again as a present (resisting) reality by reproducing the series of muscular (voluntary, but resisting) sensations required to revisit his shop. But I can only get the merman as an image (unresisting) by a train of ideal (voluntary, but unresisting) associates. The former alone $I$ do and must consider externally real. The secondary criterion of exterual reality, therefore, is $m y$ ability to reinstate resisting experiences at will.

In this secondary criterion the element of persistence included in our idea of external things seems to take its rise. In saying things are, we mean also that they continue. That is, as we have seen, we 
mean that we are able to go and find them again, and find them with the same resistance they showed when we experienced them before. To a creature without memory, reality would be simply successive resistances: but with memory as recognition comes also persistence.

The history of opinion regarding belief in objective things shows that the twofold nature of the complete criterion has been generally overlooked. Hume, ${ }^{1}$ Mill, ${ }^{2}$ Pikler, ${ }^{3}$ James, "emphasize the voluntariness (memory side) of reality. The same is seen in Mill's famous definition of external reality as "the permanent possibility of sensation." Bain," Spencer," Stout, ${ }^{7}$ Lipps, ${ }^{8}$ give corresponding emphasis to the resistance side. The latter certainly are more just, since resistance is the last test even of images which persist: but the memory side of externality just as certainly requires due recognition, and a true view means the theoretical union of the two. ${ }^{\circ}$ Of recent writers, Lipps' treatment is, in the author's opinion, the most adequate. Belief in external reality is thus seen to attend upon the whole process of perception. Its first rise follows upon the differentiation of sensations as need-filling, resisting, etc.; the feeling of massiveness and externality accompanies localization; and persistence is the feeling of repetition and association involved in sense-intuition. The element of involuntariness in sense-intuition, by which certain groups of sensations not only are but must be found together, is well pointed out by Lipps.

The objective validity of belief in external things is, of course, a question of metaphysics.

1 Treatise on Human Nature, pt. III. sects. 7, 8, and 14, and pt. IV. sect. 2. Hume's two criteria of external reality, constancy and coherence, both involve memory.

Eixam. of Hamilton, 1. chap. 11.

Psychology of Belief in Objective Existence.

4 Loc. cit., chap. XxI.

Emotions and Will, 3d ed., pp. 574-583.

- Princ. of Psychology, vol. II. pt. vII. chaps. 16-18.

Loc. cit.

8 Grundthatsachen des Seelenlebens, chap. xvir. Lipps uses the word Widerstandsgefühl for what I have called uncontrollableness in general, and Zroangsgefühl for the resistance-feeling of external reality. Ibid., p. 379.

'Cf. the writer's article in Mind, July 1891. 


\section{§6. Belief in Concepts and Thought.}

Thought-coeffcient. In conception we pass from the simple reproduction of experience to the abstracting and generalizing function of apperception. Conception, judgment, reasoning, have been already described as the successive efforts of consciousness to maintain identity throughout the diversity of new experiences. The fundamental movement, thereforewhat we may call the logical impulse-is to secure identities or partial identities, resemblances, consistencies, in its content. The demand of thought in general is agreement, consistency; its opposite is contradiction: this it cannot abide and be satisfied.

Consequently, consistency, the absence of presentative or conceptual contradiction, is the thought-coefficient of belief. Where no other coefficient conflicts, mere consistency carries intellectual assent. But by intellectual assent, it must be carefully noted, is meant formal assent, logical assent, indifference as far as the logical impulse is concerned. My belief is evidenced from the fact that I go right on and form my general class, until some inconsistency leads me to break it up.

Now in judgment and reasoning, as has been said in an earlier connection, this coefficient is made explicit. I assert my identities as logical identities. So logic is a formal science. It has no reference to what we call external fact, for that would be to call in other coefficients of belief-sensational, æsthetic, etc. And in the existential jndgment this is just what we do. The categorical expresses logical belief only; the hypothetical expresses the dependence of the logical process upon sensational or other material conditions; the existential expresses belief in these material conditions.

1 Compare the whole of chap. xIv, Senses and Intellect. 
§\%. Emotional Beliet.

No detailed argument is required to show that strong emotion has an influence on belief. So evident is this that the emotional method of persuasion is universally recognized. An idea which strongly excites us to some definite emotion, hope, fear, anger, love, is easily believed in, and the cherishing of the emotion is a means of intensifying conviction in reference to its object.

The emotional coefficient, therefore, consists, like the sensational coefficient, in intensity and uncontrollableness. While mere intensity does strengthen conviction, yet it may be questioned whether it is not mainly because it is through intensity that we lose control. As soon as we can get our emotion under our will, and can say to ourselves, "think calmly," the distorting influence of feeling disappears.

It is necessary for completeness of treatment to indicate that there are beliefs in æsthetic and moral truths; that these beliefs accompany satisfactions which arise from the gratification of corresponding æsthetic and moral impulses. What it is, however, that furnishes the gratification-constituting the æsthetic and moral coefficients-must be left till the emotions concerned are themselves discussed. ${ }^{1}$

\section{§ 8. General Conclusion on Reality and Belief.}

The consideration of the different coefficients of belief leads us to conclude that there are as many kinds of reality. There is moral and æsthetic reality no less than logical and sensational reality; and there is the same reason for believing in one that there is in another, for both rest upon the fact that our mental nature demands certain kinds of satisfaction, and we find it possible to get them. Sensational reality will not satisfy

${ }^{1}$ Chap. IX, below. 
our logical demands, for nature is often refractory and illogical. Neither will logic satisfy our moral and asthetic demands, for the logically true is often immoral and hideous. It is well, therefore, to write large the truth that logical consistency is not the whole of reality, and that the revolt of the heart against fact is often as legitimate a measure of the true in this shifting universe, as is the cold denial given by rational conviction to the vagaries of casual feeling.

Composite Realities. The outcome of our life of belief is the more or less complete adjustment of these kinds of reality to one another. We find ourselves constantly denying, minimizing, scouting the external world, as we abstract our higher selves from connection with it. Idealistic philosophy is more a revolt from the sensational coefficient in the name of the moral coefficient, than the logical system of belief which as philosophy it claims to be. Materialism, on the contrary, is the worship of the sensational coefficient as more real than any other. Religious truth either tells us which to put under and which to embrace, or bids us await a future state when all the demands upon us will be harmoniously adjudicated.

What $I$, as an individual, therefore, believe, is a composite thing, a mixture of truths representing the degree of harmony I have succeeded in reaching among things which, taken singly, I am obliged to accept. Among them the largest place is given to external or sensational reality. I bring things wherever possible to the test of sensation. No doubt this is because my connection with the external world is most intimate and direct, and the penalties of its disregard are most quick and sure. Next in practical importance is the world of logic or demonstrable truth, which holds its sway imperatively when sensation does not vote a negative. The disregard 
and violation of æsthetic, moral, and religious truth is due to the difficulty of deciding just what these coefficients are, and of disentangling them from the swarm of temporary emotional states which have not the same claim to satisfaction.

Self the Ultimate Reality. Amid the variations of composite and varying reality, the most fixed point of reference is now seen to be the feeling of self. This is as far as psychology can go with its analysis of reality. All reality is given us through our own experience and the centre of experience is self and its needs.

Existence. There are, moreover, as many kinds of existence as there are coefficients of reality. We have already seen that judgment involves belief in existence of some kind, but not always external existence. ${ }^{1}$ It may be mere mental existence (imagination-coefficient), as in the world of fiction and mythology; or ideal existence (æsthetic coefficient) ; or logical existence (thoughtcoefficient), as belief in a hypothesis; or it may be what we call "real existence" (sensational coefficient), belief in external reality. And things are constantly passing from one of these kinds of existence to another. We learn that we had mistaken its coefficient. Santa Claus passes from real existence to imaginative existence; disembodied spirits in the minds of some undergo the contrary change in the manner of their existence. ${ }^{2}$

Relation of Belief to Will. If the foregoing theory of belief be true, it is evident that belief is not the feeling of effort or volition. It is a feeling of willingness or consent, but not of will. I often consent to reality

\footnotetext{
1 Senses and Intellect, p. 286.

2 See James' interesting treatment of the Worlds of Reality, loc. cit., II. pp. $291 \mathrm{ff}$.
} 
against my will. The effect of will upon belief is really the effect of voluntary attention upon one or more of the coefficients already mentioned. Attention may intensify an image and so give greater sensational or emotional reality. It may also dwell upon and bring out certain relational connections of an image and so throw the logical coefficient on the side of those connections: it may refuse to dwell upon those relations which are distasteful. But it is not true that we can believe what we will. To say we believe what we need, is not to say we believe what we want.

There is a distinct difference in consciousness between the consent of belief and the consent of will. The consent of belief is in a measure a forced consent : it attaches to what is - to what stands in the order of things whether I consent or no. The consent of will is a forceful consent-a consent to what shall be through me. Further, in eases in which belief is brought about by desire and will, there is a subtle consciousness of inadequate evidence, until by repetition the item desired and willed no longer needs volition to give it a place in the objective series: then it is for the first time belief, but then it is no longer will.

Deflnition. Belief was above defined as the consciousness of the personal indorsement of reality. Reality we have now found to be a general term for that kind of experience which satisfies one or more of the needs of the individual. Belief in anything is then, put most generally, the consciousness of the presence of that thing as fitted to satisfy a need: and it is distinguished from the earlier unreflecting reality-feeling, which is the simple consciousness of a presence.

So far the psychology of belief and reality. The metaphysical question of reality is quite a different thing; how to account for these different coefficients and reduce them to a single truth which shall satisfy all my needs. Shall we credit them all, with Reid; or discredit them all, with Hodgson? It is not for us to say anything about it here-if we had 
anything to say-except that all our needs must stand and fall together, and no metaphysics which does justice to one coefficient only, and calls that reality, can stand before the presence of those who find some other need within them more urgent for the utterance. ${ }^{1}$

In regard to theories of belief, it may be sufficient to say that each of the coefficients mentioned above as sensational (Hume, Sully) and logical (Mill) has been considered as the principle of belief. Bain explains the state as taking its rise in the rolitional life. We believe only what we would be willing to act on. James endeavors to cover all these partial truths in a doctrine which he sums up in the words, "the most compendious formula perhaps would be that our belief and attention are the same fact." While his discussion is suggestive in detail, I am unable to bring his partial statements under a sufficiently exact principle. The account above endeavors, by the delineation of belief as a feeling arising from the successful outgo of impulse, to give it the definite standing which was not possible while it was confused, by every one, perhaps, except Brentano, with the simple fact of consciousness itself, called above reality-feeling.

Interest and Belief. A further interesting question is the relation of these two states, considered as ideal feelings, to each other. Interest is the feeling of an impulse to attend aroused by an object ; belief is the feeling of possible satisfaction for this and other impulses. Interest has a distinct future or prospective reference. If my future were forever cut off from an object, my interest in it would die away as soon as the image of it became so faint and infrequent as not to arouse a strong impulse. But, however thus cut off in the future, I would not lose my belief in such an object: for the memorycoefficient of it would last as long as memory itself, and with it the peculiar coefficient of the object's own reality. Belief, therefore, has a retrospective reference. Interest must be perpetually renewed by new impulse, new ap-

'See Lotze's subtle discussions, Metaphysics, chap. vI, and Microcosmus, bk. IX. chap. III. 
perceptive activity; belief can only be destroyed by experience which impels me to conclude that it was at first misplaced. The points of similarity between the two feelings are that they both terminate on an intellectual object, and both arise in connection with an impulsive mental outgo.

On belief in the external uorld, consult: George, loc. eit., p. 234: Paffe, Considérations sur la Sensibilité, chap. v ; Pikler, Psychology of Objective Existence; Horwicz, Psychologische Analy. sen, 6 Absch.; Stout, Physical Reality, Mind, xv. pp. 22, 545 (and Pikler), ibid., p. 394: Baldwin, Mind, 1891, p. 389; Rabier, Psychologie, chaps. XXXI, XXXII ; Mill, Exam. of Hamilton, chap. XI.

On belief in general: George, loc. cit., pp. 406-455; Ollé Laprune, La Certitude Morale; Brochard, l'Erreur; James, loc. cit., II. chap. xxI, and Princeton Review, July, 1882 ; J. S. Mill, note to James Mill's Analysis of Phenom. of Human Mind, 1. pp. 412f.; James Mill, ibid., chap. xı ; Marty, Vierteljahrschrift für wiss. Philos., 1884; Hume, Treatise, sects. 7 ff. ; Bagehot, Literary Studies, I. pp. 412f.; Renouvier, Psychologie Rationelle, II. part II ; Royce, Religious Aspect of Philosophy, chaps. Ix, x ; Bain, Emotions and Will, 3d ed., pp. 505-35 ; Sully, Sensation and Intuition, Iv ; Newman, Grammar of Assent; Mill, System of Logic, bk. I. chap. v, Dissertations, II ; Lipps, Grundthatsachen des Seelenlebens, chap. XVII ; Waitz, Lehrbuch der Psychologie, § 36 ; Rabier, Psychologie, appendix to chap. XXI ; Höffding, Outlines of Psychology, v, D.; Adamson, Encyc. Britann., 9th ed., p. 532 ; Fechner, Drei Motive und Grunde des Glaubens; Ulrici, Glauben und Wissen; (religious) Köstlin, Der Glaube ; Leibnitz, Opera Philosophica, ed. Erdmann, pp. 442f.; Bouillier, La Vrai Conscience, chap. XIII ; Brentano, Psychologie, I., bk. II. chap. vur ; Sully, Outlines, p. 397f., and Illusions, chap. $\mathbf{x}$; Perez, l'Education des le Berceau, part v. chap. III.

Further Problems for Study:

Interest of recognition;

Are there disinterested interests ?

Educational value of interest;

Varieties of interest-indifference, enthnsiasm, etc.:

Varieties of belief-hesitation, perplexity, etc.;

Theory of probabilities ;

Selective aspect of interest and belief ;

Interest in the disgusting, dangerous, awful, etc. 


\section{SPECIAL IDEAL FEELINGS.}

QUALITY, OR KINDS : EMOTIONS.

\section{CHAPTER VIII.}

\section{DIVISION : PRESENTATIVE EMOTIONS.}

\section{$\S 1$. Division.}

General Nature and Characters. Special ideal feelings or emotions have already been given their place among the general phenomena of sensibility. They are the feelings which arise in connection with different phases of intellectual activity, as far as these feelings stand clearly distinguished in consciousness from one another. They are qualitatively different (hope and fear, for example), as sensations (sound, taste) are qualitatively different.

Besides distinctions of quality among emotions, we are able also to predicate of them quantity (or intensity), duration (time-relations), and tone (pleasure and pain), reaching the same four characters which we found to be present in sensations. ${ }^{1}$

The investigation of the emotions, it is at once seen, if the analogy from sensation is of any worth, must proceed empirically. We have no way of finding how many kinds of sensations there are, except by noting those which we actually feel : so we have no high road of certainty to an enumeration or classification of the emotions. What actual emotions do we experience? - is the question we must answer. The search after an exhaustive classification of the emotions is accord- 
ingly not worth its cost, except in as far as it leads us to inquire what emotions our geueral mental theory would lead us to expect.

Kinds. Upon examination, states of ideal sensibility fall into two classes, which may be called respectively emotions of activity and emotions of content, i.e., first, feelings of the operation of the apperceptive function without reference to what it operates upon; and second, feelings excited by the particular object upon which the intellect operates. These classes of ideal feeling suggest themselves for separate treatment.

This distinction is essentially the same as the current German (Herbartian) division of emotions into formal and material. ${ }^{1}$ The terms of the text are preferred because they accord better with the terms already used in earlier connections, and also because the use of the distinetion between "formal and material" carries metaphysical associations unnecessary here. To adopt that terminology would also prejudice the detailed treatment below in favor of the Herbartian theory of feeling, to which strong exception may be taken. It may be noted that of the common ideal feelings already treated of, interest belungs to the emotions of activity, and the feelings of reality and belief to those of content.

\section{§ 2. EMotions of Activitr."}

It has already been seen that all mental activities reside, that all apperceptive processus happen, in the attention: hence the great class of emotions of activity cluster round the different phases of the attentive life. These feelings again fall into two classes, which we may call feelings of degree of adjustment, and feelings of function, or activity proper.

1 See Nahlowsky, Das Gefühlsleben, pp. 49-51; also Waitz and Volkmann, in loc., and in English, Ward.

' Cf. especially the clear treatment of Waitz, Lehrbuch d. Psych., pp. $395 \mathrm{ff}$. 
Emotions of Adjustment. It is an easy matter to get these feelings experimentally. Attention to successive stimuli-say sounds-following one another in very rapid succession, soon grows painful as a feeling of distraction or confusion. The attention cannot adjust and readjust itself in time to bring order into its stimulations. On the contrary, when there is an evenmeasured flow in the appeals to which the attention is open, we have a class of feelings of abstraction or clearness. Again, a stimulus may be so slight, vague, dim, as to lead to violent concentration upon it, giving feelings of contraction or effort; and again, we often have the consciousness of unusual breadth of view, comprehensiveness of range, expansion or ease.

It is seen that these emotions, given thus in pairs, indicate roughly a distinction between what is called sensory and ideal attention. The first of each pair-distraction, abstraction, contraction, expansion-refer to the adjustment of the attention itself with the muscular feelings it includes. A number of sounds distract, a continued musical chord abstracts, a microscopic preparation contracts, and a landscape expands, one's attention; and the different feelings are very distinct. An outstanding feeling of good adjustment is that of rhythm, based upon successive movements of the attention. On the other hand, the second named in each pair refer more particularly to the apperceptive or thought process which is brought about in the corresponding state of the attention. Many reasons confuse a subject, but the discovery of harmony among them clears it up; but I may make an effort to understand it better, and my success leads to a feeling of ease. These feelings become generalized in the common ideal feelings already treated, i.e., doubt and belief.

There are a great many subordinate varieties of these feelings of adjustment, but the enumeration of them is not 
necessary: such are fatigue, sluggishness, opposition, seeking, finling, success, failure, hesitation, perplexity, uncertainty. As said before, such enumerations are quite empirical and incomplete. Excellent detailed treatment from the Herbartian standpoint is Nahlowsky's. ${ }^{1}$ For theoretical considerations, the student may consult Ward.'

Fmotions of Function. Although the line of distinction is inexact, this class of emotions is conveniently separated from the foregoing. They are feelings of the apperceptive process, as far as it is felt in operation; still, however, apart from the nature of the particular object of its operation. The going-out of the attention may be felt as freshness, triumph, eagerness, alertness, hope, courage, aspiration, elation; or as hesitation, indecision, anxiety, timidity, melancholy, irritation, fear. The former great class may be known in general as emotions of exaltation, and the latter as emotions of depression.

A division has been attempted of these, as of other emotions, into those having present, past, and future reference; but the details are plain enough to suggest themselves to the reader. Moreover, as far as they are purely feelings of function, and do not vary with the object referred to as past, present, or future, they are only three, all of which have been suggested under the head of common ideal feeling, i.e., feeling of recognition of that which is past, of presence in regard to that which is now, and of expectation of that which is future. If more than mere time-form is meant, and if such feelings have qualitative differences which mark them as past, present, or future, then they belong under the head of feelings of content, or "material " feelings.

§ 3. Enotions of Content, I.E., Having Reference to OBJECTS.

The great body of our emotional states have not the fixity and exact determination of emotions of activity. The so-called objective or qualitative emotions vary

'Loc. cit., pp. 85-130. See ulso Dewey, Psychology, pt. II. chap. xII.

Encyc. Britann., art. Pychology. 
with all the varieties of combination in which objects or objective truths may take shape. Accordingly, no exhaustive classification in detail will be attempted. Perhaps the most convenient, as the most evident, division of these emotions, is based upon distinctions among their objects, as regards the kind of belief-coefficient which they involve.

Proceeding on this plan we may distinguish presen tative from relational emotions, and under the presentative order we find, first, a great class which refer exclusively to self, terminate on the ego; for example, pride. These we may call self-emotions, after analogy with the more affective kinds of sensation, which, it will be remembered, have most direct value as reflecting the subjective side of sense-experience. Another class, under the presentative type, depend upon the relation of the object of the emotion to one's self, as fear, etc. These we may call objective emotions, after analogy with the knowledge element in sensation. Relational emotions, on the other hand, terminate upon objects which have certain complexities in themselves apart from their connection with the individual. The presentative emotions carry belief in the sensational or memory coefficient; the relational, in the logical coefficient.

Further, under the objective emotions, we may distinguish the expressive from the sympathetic. The former indicate a reaction in consciousness outward as an expression of personal feeling; and the latter indicate a similar reaction, which is now sufficiently described by the term sympathy. Again, feelings of relation fall into so-called logical and conceptual feelings.

The divisions thus indicated may be presented to the eye in the following table:

$$
\begin{gathered}
\text { Emotions of } \\
\text { Content }
\end{gathered}\left\{\begin{array}{l}
\text { Presentative }\left\{\begin{array} { l } 
{ \text { Self } } \\
{ \text { objective } }
\end{array} \left\{\begin{array}{l}
\text { Expressive } \\
\text { Sympathetic. }
\end{array}\right.\right. \\
\text { Relational } \\
\begin{array}{l}
\text { Logical } \\
\text { Conceptual }
\end{array}
\end{array}\right.
$$




\section{SELF-EMOTIONS.}

The emotions which terminate on one's self must be clearly distinguished from the feeling proper of self. The feeling of self underlies all other forms of consciousness wheu self-consciousness has once arisen. Assuming this to be so, whatever self may be, we find that the contemplation of self, when it becomes the object of our reflection, arouses certain spontaneous and peculiar forms of emotional excitement. These are the emotions of self.

Such emotions attend either an exalted estimate of one's own person or possessions, or, on the other hand, a depreciatory estimate. The former we may call emotions of pride, and the latter emotions of humility. Looked at casually, emotions of pride include the states ordinarily called pride, vanity, haughtiness, conceit, superiority, complacency, arrogance, self-confidence, frowardness, etc.; and under emotions of humility we have humility, modesty, self-debasement, self-distrust, inferiority, bashfulness, meanness of spirit, weakness, poverty, shame, etc.

In different individuals these emotions have habitual stimulation in very varying circumstances. One vainglorious mortal dwells always upon his past exploits; another, on the mighty deeds he is going to perform. One humble spirit bears always in mind the weakness of his earlier or present endeavors; another lives in constant dread that an occasion may arise in which his real shortcomings will become evident. Moreover, besides the common object of them all, self, viewed in a narrow sense, these emotions attach very broadly to anything in which one's interest is wrapped up, or for which he is in any way responsible. Without discussing the question as to whether the extent of these feelings justifies our extending the notion of self to include all the objective personal interests of the man, it is still true that his 
self-feelings overflow, as Hume maintained, and attach themselves to all objects with which he is closely and habitually associated. A man grows proud of his college, his boarding-house, even of the valor of his enemies; ashamed of his associates, of the shabby dress of his grocer, of the venality of his political adversaries. Give me a real interest in anything whatever, and it becomes mine in an emotional sense : its fate affects me in the same way, though not to the same degree, as a similar fate to myself.

Self-emotions as Egoistic. This transfer of emotion from the strict self-object is seen at once to be due to the nature of interest as an egoistic feeling. If $\mathrm{my}$ pride attaches only to what I am deeply interested in, it is. because my interest represents the outcome of my own selective, habitual, and truest activities. In worshipping what is closely connected with myself, I am worshipping. largely what I have made or done-in short, my own power, or myself. The interest of mere suggestion, of curiosity, the exploring interest, does not carry my emotion of pride or humility; it is perfectly indifferent as far as its object is concerned, unless by my exploration I discover some deeper connection between this object and myself. The bearing of this truth-the essential egoism of this whole class of feelings-upon the sympathetic emotions is noted below.

\section{§ 5. ObJective Emotions.}

The objective emotions have already been characterized; they are so called to indicate that their main reference is to an object. As feelings, they are subjective states, but. they arise as differentiated qualitative states; and this differentiation seems to depend in some way upon the. relation of self to the objects which excite them respec- 
tively. But the idea of self, as itself an object presented in relation to the thing on which the emotion terminates, is not necessarily present. Children show fear, anger, etc., before they have the notion of self. The object of the emotion does sustain a relation in adult conception to self, and the emotion which was purely instinctive (presentative) at first thus becomes reasonable (representative). But the fact that the same emotion may not have a conscious self-reference shows that such a reference is not one of its essential conditions.

This case illustrates the growth of consciousness in connection with the nervous system. What in the child represents a natural inherited raction upon special kinds of presentations, is prophetic and anticipatory of conscious means to ends in the man: a nervous adaptation is confirmed and reaffirmed by a conscious adaptation. Facts of this kind favor the theory that the nervous system itself represents intelligent adaptation, either through the consciousness of its progenitors, or through the teleological principle of its own development.

The position, therefore, is mistaken that the fundamental objective emotions have necessarily a conscious self-reference. The truth is that they come to have such a reference. We would expect this from the falsity already pointed out of the idealistic position that knowledge of an object necessarily implies the antithesis of subject and object. Of course, if one hold to the latter position in regard to presentations, he must hold the former in reference to presentative emotions. Hume's celebrated distinction between the object of an emotion and its cause' is true only for the adult reflective consciousness, in which the purpose of the emotion, at first only biological, has become a conscious psychological end as well. ${ }^{2}$

1 Treatise, bk. II. pt. I. sect. 2.

- Schneider makes this distinction clear by showing that some feelings begin as purely biological (fear, anger) and end by being purely mental (dread, hate). He designates these perception (Wahrnehmungs). and representation (Vorstellungs) feelings, respectively, as distinguished from purely physical (Empfindungs) and purely formal (Gedanken) feelings. The class called presentative in the text corresponds to bis: two classes, i.e., perception and representation feelings. Schneider, Thierische Wille, pp. 95-121. 


\section{§ 6. Expressive Emotions.}

These emotions, further, find their place in the reactive consciousness, as both the study of children and adult reflection teach us. They rise in child-life before volition becomes prominent. Consequently the phrase expressive emotion serves best to distinguish them. They are an expression of the reaction or behavior of consciousness when given objects are presented. They represent the reactive, outgoing side of consciousness, as the affective emotions or feelings of self represent the receptive or reflective side.

Looked at from this point of view, emotions rest upon impulses, ${ }^{1}$ and exhibit the two great directions which have already appeared in impulse, i.e., toward or from an object as fitted to satisfy, or the contrary. Careful distinction in terminology-more careful and exact, no doubt, than the facts warrant-gives over the active, impelling factor in a state of high emotion to impulse, and reserves for emotion only the mental excitement, agitation, felt disturbance of consciousness. This, at any rate, serves to cover both aspects of the case, and gives us a terminology which may be consistently maintained.

Having in view, therefore, the direction of the impulses which the expressive emotions accompany, we may distinguish emotions of attraction from emotions of repulsion.

The treatment of the impulses which express higher ideal reactions-in distinction from sensuous impulseis now reserved.

It is accordingly the impulse which is really expressive, the emotion being the inner or conscious side of that expression. We shall see below that the question is an interesting and important one, whether the facial and general muscular 
movements which are ordinarily known as movements of emotional expression are not historically, after all, the remains of useful motor reactions, i.e., the channels of impulse. The establishment of such a theory by the evolutionists would go to show that emotion is but the consciousness that there is impulse, and that it is expressing itself.

Emotions of Attraction. Under the general head of attraction, we may include all tendencies toward au object or individual, or satisfaction in its presence; from the slight feeling of approval to the boisterous expression of social enjoyment, or to the quieter but stronger movings of affection and love. And the progress of this emotion in degree and closeness of attachment is an interesting and typical chapter in the natural history of feeling.

Beginning with interest-the emotion of simple attention-an object becomes attractive as it comes into clear relation with one's self. Both simple association, by the egoistic reference already remarked upon, and increasing knowledge of attractive qualities in the thing in question, tend to increase its attracting force. Further, any effort which may have been put forth in connection with such an object increases its hold upon us, and, by strengthening our interest, makes its presence a matter of need.

In this increased attractiveness of an object, however, we discriminate clearly between persons and things. Familiarity with things always leads to attachment to them, simply by association and interest. If the thing is useful we become further attached to it; if it turn out useless, we simply neglect it; but it still has its place in its interesting environment. But things never arouse in us the opposite, repellant emotion, except by some kind of association with persons.

In the case of persons, on the other hand, the simple attachment which now becomes, in its earliest form, admiration, passes over, on further acquaintance with the 
object, into a more positive and vigorous emotion, Strengthen the ties of association and self-relation (kinship, partnership, etc.) sufficiently, and the emotion of attachment becomes affection and love. There is a line in the growth of the emotion of attraction beyond which all revelations of character or action, however damaging, only deepen and strengthen the earlier tie. But if this line has not already been reached when damaging discoveries are made, if the attractive emotion has only reached the stage of admiration arising from intellectual interest and casual association-then there comes a revulsion to emotion of repulsion.

Around these three stages in the growth of emotions of attraction, the varieties of such feelings may be grouped. Admiration, the feeling of deep interest in persons, is veneration when its object is elderly, superior, or of high rank; awe, when it is obscurely grand and imposing. Attachment, the feeling of close association with and dependence upon persons and things, has distinct colorings, when felt toward inanimate objects, animals, inferior or superior persons, etc. Affection, the feeling of profound attraction toward persons, arising from the deeper ties of family or common life-interests, parallel opinions and aims, or congenial dispositions, takes on innumerable forms known by name as distinct emotions: feelings of confidence, patience, security, help, congratulation, self-surrender, self-denial, tenderness-in short, all the infinite emotional phases, of past, present, and future reference, which poets have sung and women have felt since one human heart first learned to enlarge its borders to include another.

All such feelings of attractiveness take on peculiar qualities when their objects are matters of future or of past time. The belief-coefficient may be a representative in distinction from the sensational (presentative) one, carrying the force of the future as well as of 
memory: these emotions are then called hope and joy respectively.

It shonld be remarked that the place of sympathetic emotion in feelings of attraction cannot be here discussed. It is a topic for future treatment; but that it bears a large part in the more intense phases of affection there can be no doubt. It must not be supposed, further, that there are no other emotional elements than the attracting motive in the feelings here mentioned. Each one of them is exceedingly complex. They are placed under the "attractive" eategory, to emphasize the kind of impulse to which they attach. 'The minute aualytic treatment of each would be an extended inquiry, largely barren of theoretical or useful results.

Emotions of Repulsion. The repelling impulses also supply us with a group of emotions of enormous range and importance. What has been said about the development of the feeling of attraction applies with some modification to this class also. Simple interest and some knowledge is necessary to induce the feeling of unattractiveness in the first instance; it grows to be objectionableness in things (mainly) or persons. The feelings toward things do not pass into stronger emotion except through association with persons. But with persons it passes into distaste, a positive feeling which becomes intense in abhorrence. At any stage, except that of extreme repulsion, an attracting motive-kinship, pride, intellectual admiration, etc.-may assert itself so strongly as to cause a revulsion of feeling over to the attractive side: and attachments thus formed are often most lasting and intense.

Many modifications of the so-called feeling of objectionableness might be mentioned: feelings of inferiority, of poor breeding, of bad faith, disdain, distrust, etc. So positive distaste may take form as impatience, scorn, rebellion, impertinence, malice, vengeance, present fear, anger, hatred, etc. And abhorrence has varieties in detestation, contempt, disgust, loathing, etc. 
All the emotions of this class also get generalized under coefficients of future and memory reality respectively, and become the opposites of hope and joy, i.e., fear (or, more properly, dread) and sorrow.

Exhaustive enumerations here also are unnecessary because both unprofitable, and impossible. The further question of interest remaining is that of the possible reduction of all varieties of expressive emotion to a few simpler kinds of reactive feeling. How far such a task is promising is indicated below in connection with ideal impulses, where the question can be approached to better advantage. It would be an interesting and profitable exercise in reflective analysis, for teachers to suggest emotions not hitherto mentioned, and lead students to attempt to assign them proper position in one of these or later categories. The physical basis of these emotions is also deferred.

\section{§\%. Sympathetic Emotions.}

The second division of presentative emotions has been called sympathetic. The word " sympathy," in ordinary usage, signifies the emotion which is called out by the intelligence of such good or bad fortune to others as sustains no immediate connection with our own. The ordinary popular understanding at the outset is that any feeling of advantage and disadvantage to one's self is different and apart from so-called pure sympathy or fellow-feeling.

An adequate psychological analysis of the conditions of sympathy seems to yield the following results :

1. It is aroused by states clearly pleasurable or painful. There is no occasion for sympathy with one who does not need it; that is, with one who is not in a state of positive feeling, good or bad. Further, the study of the first sympathies of children shows that they extend to

1 Only the painful causes of sympathy will be considered in detail : this accords with the popular usage. The same considerations apply, however, to the pleasurable exercise of sympathy. 
things as well as to persons, and only gradually get narrowed down to sensible objects. Sympathy as au emotion is shown before the child makes any distinction between things that feel aud those that do not.' But whatever the object be, the emotion is called forth only by such happenings as have before excited the child's own feelings of pleasure or pain.

2. Some degree of interest is necessary to sympathy. The confirmation of this appears broadly in every-day experience. I read in the morning paper that thousands of people perished in a Chinese flood, and the cup of coffee that follows it up is much more important to me than their bereaved families. But a single death in my own community makes me at once solicitous in reference to the deceased man's relatives. Yet mere exploring interest, when it comes upon suffering, always starts the sympathetic feeling.

3. My sympathy is in a rough way proportionate to the nearness of the individual's connection with myself. This, again, needs no detailed proof : if my brother break his leg, I feel more sympathy than if a casual comrade meet the same misfortune; and the difference is greater still if the latter be a servant or a favorite horse.

4. Sympathy is aroused, not merely by real beings, but by any idea of suffering. It is not necessary that we believe in the object of our sympathies. Pictures in memory win our sympathy, imaginations in fiction arouse it, vague forebodings of misfortune to others excite it. Whenever there arises in consciousness an idea of a sensible creature-be he fact, memory, fancy, illusion, reality in any of its kinds, that is, be he a possibility in any form-his fortune as suffering or enjoying moves our sympathy.

${ }^{1}$ Darwin observed sympathy in his child six months and eleven days old. Mind, II. p. 289. 
This is true in spite of our efforts, often successful as they are, to suppress sympathetic emotion by dwelling upon the unreality or ill-desert of the subject of it. Little Dorrit will move some readers, in spite of their sense that the character is fictitious. We all feel the stirrings of fellow-feeling for the condemned criminal, even though we be convinced of the justice of his sentence. In cases in which we do suppress the emotion, it is by getting rid of the idea, turning the attention to something else, exciting some new interest, that we do it; not by depriving the subject in question, the idea of suffering, of its force to affect us.

Definition of Sympathy. It is plain, if these points be true, that sympathy is an emotion aroused by any presentation which suggests suffering.

In this definition several further considerations are involved. By the use of the word "suggestion" an important distinction is intended between the object on which sympathy terminates and that by which it is caused. A suggestion is a stimulating idea which is brought into consciousness from without, or comes by an association, in such a way that it does not belong in the course of my real life. A suggested pain, for example, is a pain which I am led to think of, but which I am not really suffering. Suggested suffering has not the present coefficient of pain, but only a remembered coefficient of pain. Suggested suffering, therefore, is the idea of pain as far as it differs in consciousness from the actual pain of the experience presented.

But the question arises: Does such a suggestion excite sympathy? Suppose a cruel father who punishes his child by pinching, the presentation of the father may suggest pain to the child; but this does not seem. to be sympathy - it may be fear, or memory of pain. Yet, on looking closer and observing children, we find 
that if the father take the attitude which the pain before accompanied, real sympathy is excited. Let him pinch a piece of wood, paper, even his own finger, and the child a year old gives clear expression to its sympathetic emotion. The child does not need the notion of another person who suffers, nor even of another object that suffers; he only needs two things: first, a presentation which suggests vivid pain, and second, the absence of the coefficient of reality which his own suffering had. In other words, the emotion of sympathy does not require an object at all. It acquires an object, and then maintains itself by the emphasis of this object; but in the first place it attaches to any convenient presentation in close connection with its exciting cause.

This seems at first sight a remarkable statement, but it is borne out even by adult experience. Personally, the writer finds it impossible to think of suffering in the most abstract and general way without feeling the beginnings of sympathy. The simple thought of a bloody knife excites the emotion, when the only object is found to be the fragmentary glimpses of deat bodies, butchers' stalls, and squealing pigs, which stand for murder. And further, there seems to be no difference in the conscious feeling of remembered pain and present sympathy, except a vague outward reference, which means only that it is not real pain now in me.

The foregoing assumes, evidently, that there is a difference of some kind between "real present pain" and the pain which is suggested or remembered. It is sometimes held that a suggestion or memory of pain is real pain. This is true if in each case we mean only the tone, not the qualitative emotional state which it accompanies. But "suffering" is always a particular kind of suffering, not pain in general ; and the particular qualitative coefficient of (say) a toothache, as distinguished from remorse, is clear enough. In the suggested suffering this qualitative element is wanting or very confused.

Kinds of Sympathetic Suggestion. We may sympathize, therefore, without sympathizing with anything, and at first this is the experience of the young child. 
But its sympathy gets an object, and, by getting it, develops and maintains itself. The fact that the suggesting presentation is generally the same as the suffering creature tends to give stability to the object of the emotion. Then there arises the apprehension of the physical signs of suffering, for which the child inherits in some few instances a direct susceptibility; and these carry the objective reference of the sympathies over to themselves. Indeed, it is probable that the first suggestions of suffering come from the facial and vocal expressions of others. Imitation also leads to the copying of the movements of emotional expression of others, and this reacts to suggest the appropriate emotions in the child himself. ${ }^{1}$

Development of Sympathy. The rise of sympathetic emotion may be described in view of the foregoing. Considering only the feeling elements, in the light of what has been said of the intellectual conditions of each, we may distinguish three stages in this development, i.e., affect, interest, concern. The affect we understand to be a simple present state of feeling considered as having motive force: say a present pain. Affects become strongly associated with presentations, and this association is a process occurring in the attention; the exercise of the attention then excites interest. Interest, further, as far as it arises in connection with pleasures and pains remembered, introduces concern, i.e., sympathy become definite as terminating on a distinct personal object.

Altruistic Element in Sympathy. The much-discussed question of egoism $v s$. altruism in the sympathetic

${ }^{1}$ Cf. Sully, Outlines of Psychology, pp. 508 ff. Spinoza had a glimpse of this ; be says (Ethics, IIr. demonstr. 27): "The idea of an external body imagined by us will imply an affection of our own body like to that of the external body." He held, however, that "we must imagine any one like ourselves to be affected with any emotion in order that we may be affected with the said emotion." 
emotion may receive partial consideration here. If it be true that suggested suffering excites sympathy, and that it is only suggested suffering that excites it, uamely, suffering not felt to be present as real suffering is, and for that reason attributed, when knowledge is sufficiently advanced, to some one else-then we must believe that sympathy is not entirely egoistic. Suggested suffering is at first neither egoistic nor altruistic, because neither the ego nor the alter exists in consciousness when sympathy at first arises. 'The reference of real pain to self, and of suggested pain to another, seem to be both late acquirements. But as it is true that the child gets his external objects clearly presented-especially his external personal objects-before he clearly presents himself, so sympathy must be a conscious emotional motive before self-seeking is.

Here, again, it is necessary to draw a careful distinction between the origin of sympathy and its relation to volition. Sympathetic emotion arises from experiences of personal pleasure and pain ; of this there seems to be no doubt. But in acting from sympathetic impulses, the object of the sympathy is the end set before consciousness, not the attainment of pleasure or the avoidance of pain. Suggested pain in others may lead to a course of conduct directly opposed to the course most pleasurable to one's self ; that is, two suggestions, one selfish, the other sympathetic, may arise in direct opposition to each other, and the sympathetic suggestion may win the day.

Nervous biological conditions with passive or reactive consciousness, therefore, account sufficiently for the origin of sympathy as an emotion. But it is only the "psychologist's fallacy" to make such sympathy of ethical worth, on the one

1 "It is psychologically without meaning," says Höffding, " to speak of a native egoism, if by egoism is understood the conscious setting of the weal and woe of others below our own." Outlines of Psychology, pp. 242,243 . Stephen says there is fellow-feeling before there is knowledge of others' feeling (Science of Ethics, chap. vi. §2); but knowledge of one's own feeling, as one's own, is equally absent. 
hand, with Bain ${ }^{1}$ and Spencer, by converting it into the selfconscious sympathy which sees self hidden "in the shoes" of the ostensible object of the kindly emotion; or with Green," on the other hand, to make all sympathy a matter of the rational construction of an end, which turns out after all to be self. The ethical aspects of the case receive consideration below. ${ }^{3}$

Varieties of Sympathetic Emotion. A large number of varieties or shades of emotion may be classed as sympathetic, i.e., kindness, benevolence, charitableness, etc. When felt toward an equal in character or station, we call it congratulation, fellow-feeling, fellow-suffering, companionship, common well or ill desert, solicitude, heartache; toward an inferior, compassion, pity, mercy ; toward one much superior, it approaches awe, but differs from it in an unnamable way.

There is also malignant sympathy, i.e., pleasure in another's pain. It is a compound emotional state, in which the pain of suggested suffering is overwhelmed by the gratification of powerful unworthy emotions, such as hate, revenge, self-assertion, etc. Ordinarily it carries a distinct element of disquiet and self-reproof ; yet in some natures it seems to reach pure malignity. This feeling oftener manifests itself as dissatisfaction at another's success or happiness.

${ }^{1}$ Emotions and Will, ch. vr. Both Bain and Spencer hold that sympathy has arisen in connection with the " gregarious instinct," i.e., the best conditions for life have been those secured by flocking or herding, and in this way the connection between an animal's own safety or comfort and that of other animals was established.

2 Proleg. to Ethics, pp. $211 \mathrm{f}$. It is only a repetition of the fallacy of Green's main position that all feeling involves knowledge of self. He says: "If imagination (suggestion) is merely the return of feeling in fainter form, no one can imagine any feeling, any more than he can originally feel it, except as his onon." Introd. to Hume, п. p. 28. Sympathy, to Green, involves a " conceived identity, or unity in difference, between the spectator's own person and the person of the other." Ibid., II. 43, 44.

${ }^{3}$ Chap. IX., § 7 . 
Social Foeling.' The further generalization of the idea of personality to which developed sympathy attaches gives the emotion a broader reference. Social feeling is sympathetic emotion as it attaches to man in general. It can only arise after the conception of man is reached, of man as a multiplication of particular men like myself. As long as men were not considered all "like myself," but some slaves, some barbarians, some Gentiles-only a few Greeks or Hebrews-social feeling had only the range of the class or race in the midst of which it arose. Particular forms are feelings of equality, justice and injustice, rights, political and patriotic feelings, etc. Also under this head should be included feelings of rivalry, emulation, jealousy, ambition, competition, love of fame or reputation, sensitiveness-all the emotions, in fact, which arise from the association of man with man in social life. Intense pleasure and pain both tend, it may also be remarked, to sociability and communicativeness.

\section{§ 8. Representative Emotions.}

Presentative pass over into representative emotions when the object is itself representative, i.e., a memory, imagination, reproduction of any kind. It is sufficient to say here that the emotion aroused by a reproduction is the same in kind as that of the original presentation. They are prevailingly, however, of much lower intensity. The time element which they involve also gives them a new coloring: the joys of memory are different from the joys of the present or the future. This means that the substitution of the memory for the sensational coefficient of belief has its meaning in the emotional consciousness.

1 On the relation of the sympathetic and social emotions to the egoistic (self) emotions, see Ward, loc. cit. 
So also, as Schneider claims, ${ }^{1}$ the emotion accompanying simple existential and categorical judgments passed on objects is still the same in kind as the presentative. The judgment is only the explicit belief-form of the synthesis of perception, and the object fills consciousness just as a percept does. I fear burglars more as I talk about them and describe their murderous deeds. There is here a new coloring of emotion, however, given by the consciousness of the absence of sensational reality. We hold ourselves aloof from the emotion, as it were, and deny its right to arise. The feeling of activity strives with the feeling of content. This Schneider overlooks, and consequently has no adequate basis for the feelings of relation treated in the next chapter-feelings of which the forms themselves of conceptual relation have become the objects.

On objective and sympathetic emotion, consult : (development of) Perez, l'Education dès le Berceau, part IV ; Höffding, Outlines, VI, $\mathrm{B}$ and $\mathrm{C}$; Horwicz, Psychologische Analysen, in loc.; Hodgson, Theory of Practice, vol. I, in loc.; Volkmann, Lehrbuch, 135-138; (sympathy) Sully, Outlines, pp. 508f.; Bain, Emotions and Will, part I. chaps. IV-XI ; Drbal, Lehrbuch, \$ 114-125 ; Carpenter, Mental Physiology, bk. I. chap. vII ; McCosh, Emotions; see also the works on Ethics for discussions of Sympathy.

On social and personal feeling, see refs. given by Dewey, Psychology, p. 346.

Further Problems for Study :

Development of sympathy ;

The ethical aspects of sympathy.

1 Thierische Wille, pp. 120,121. Schneider draws an argument for the identity of emotion as it progresses from perception to thought, from the sameness of its physical expression; for example, disgust. 


\section{CHAPTER IX.}

\section{EMOTIONS OF RELATION.}

ThE higher reaches of apperception in conception, judgment, and thought, give rise also to characteristic emotional states. The fundamental act of attention as relating function gives most general coloring to this class of feelings, and from it they also derive their name, relational feelings. The divisions of these emotions we should expect to correspond to the kinds of relation discoverable in the forms of judgment. These have already been classified above,' and while the feelings to which they give rise are, like feelings generally, less clearly differentiated, and their nomenclature quite undeveloped, a general correspondence may be easily discovered in consciousness. In the description which follows, however, no attempt at such an exhaustive parallel is attempted.

At the outset, three very distinct kinds of emotional experience may be distinguished: intellectual or logical feelings, ethical or feelings of right and wrong, and cesthetic or feelings of the beautiful. The latter two may be further classed as conceptual feelings.

It may be observed that these feelings are distinguished clearly as a whole, though not always clearly in particular cases, from the emotions of activity already described. Here we have emotions attaching to content, but this content. is constructed in relations, i.e., the relations are part of the content. Activity-feelings arise from the activity itself, and presentative feelings arise from objects in their more isolated and unrelated character. For example, I attend successively

1 Senses and Iniellect, chap. xIV. §4. 
to two tigers in a cage-emotion of activity. I discover that they are similarly and beautifully marked-emotion of relation. I am informed that one of them is at liberty-presentative emotion of fear.

Recent work in comparative psychology is emphasizing the line of demarcation between the intellectual processes involved in presentative and in relational emotion. We have already had occasion to note the distinction between the reactive or receptive form of consciousness-that of perception, memory, passive imagination, implicit judgment; and the active consciousness in which the data of free reproduction are constructed under the lead of explicit selection-constructive imagination, thought. The former is enlarged upon by Romanes," and its products called "recepts" in contrast to concepts proper. Prof. Lloyd Morgan ${ }^{2}$ marks the same distinction by the terms "constructs" (percepts) and "isolates" (concepts). Both writers point out the "composite" generalized character of passive mental construction and draw the limit of animal intelligence just short of active conception. This distinction is slighted, as we have seen, by Schneider and other evolution writers.

\section{§1. Logrcal Emotrons.}

By distinguishing the more fundamental emotions of relation as logical, we intend to point out those to which the coefficient of thought-belief attaches: those which attend upon the various acts of judgment. First, we find a class of feelings arising from bare relationship as itself the object of consciousness, i.e., feelings of reasonableness and unreasonableness, of contradiction, of logical satisfaction, of tendencies of thought,' of ignorance, of the unknown, the mysterious, the inscrutable, feelings of the conclusiveness of argument, of the hypothetical, of the inconclusive, etc. These feelings are in close affinity with the great class-feelings already described as doubt and belief.

1 Mental Evolution in Man, chap. III.

"Animal Life and Intelligence, chaps. vIII and Ix.

s Cf. James' interesting description of Feelings of Tendency, loc. cit., I. pp. 249 ff. 
Connected with time-relations we have what may be called time-emotions, i.e., anticipation, prophecy, presentiment, hope, attaching to the conception of future time; retrospection, revery, musing, regret, feeling of the irremediable, of opportunity lost or improved, attaching to the conception of the past; and routine, surprise, amazement, astonishment, present opportunity, hasty decision, attaching to the idea of the immediate present.

Space-relations also are reflected in emotional states: feelings of distance, moral remoteness or nearness, grandeur, pettiness, mental vacancy, besides the ordinary sensuous feelings of spacial relations.

Other relationships give us feelings of coexistence or the contrary, i.e., communion, community, company, loneliness ; of quantity, i.e., importance, insignificance, greatness, abundance, economy, paucity, poverty, completeness and incompleteness; of identity, ${ }^{1}$ i.e., sameness, resemblance, difference, contrast, quality; of finess, i.e., utility, uselessness, adequacy, insufficiency, redundancy, congruity and incongruity, suitableness, adaptation, means and end; of objective power, i.e., agency, destructiveness, might, fearfuiness.

The peculiarity of this whole class consists in the conscious explicitness of the act of relating. Judgment has been distinguished from conception and imagination by this very feature. Yet as there is every degree of progress from the more mechanical union of factors in the pictures of passive imagination, to the clear cousciousness of relation as found in judgment; so these

1 It is under this head that the emotional effectiveness of rhetorical expression and lllustration arises, such as the pleasure of a good figureanalogy, simile, hyperbole. Under this head, also, is the feeling of logical identification, or pleasure of the fitting of new materials into the accustomed form of our reasoning, which the Herbartians make the essence of logical feeling as a whole (Waitz, Lehrbuch, p. 300). This feeling of logical framework or form is often present in the wildest vagaries of dream-life. 
feelings vary from a most to a least degree of explicitness in this respect. As might be anticipated, further, there is a class of emotions attaching peculiarly to the least evident degree of relationship, as it appears in the apperceptive process before it reaches conscious assertion in judgment. These we may now consider.

\section{§2. Conceptual Emotions.}

The progress of the intellect from the involuntary combinations of fancy to the free constructions of imagination and conception has already been depicted. This progress is a matter of feeling also; the feeling of enlargement of range, emancipation, constructive capacity, which is covered in popular language by the phrase. getting or having ideals. If my imagination builds up for me something more pure and satisfying in any particular-form, color, use-I say that result approaches more to my ideal in that direction. If, again, I set my. self to draw up a system of philosophy, I express my satisfaction at each turn of its development by saying it. tends toward my ideal of a system : and I reverence a character more because, as I think, it more nearly embodies my ideal of a man. So in all construction whatever, besides the feeling of the extent of actual. construction, there is a feeling of further possible construction-construction beyond what I have done, yet in the line of what I have done.

\section{§3. Construction of Ideals.}

The process of constructive imagination has been described. ${ }^{1}$ It is the machinery by which ideals are produced. It is only necessary here to give the elements, before pointed out, their proper place in the scheme of feelings.

1 Senses and Intellect, pp. 226-243.

2 Ibid., pp. 227 f. 
The appetence or moving force which impels a scientist or artist to produce is the impulsive principle of need now found to underlie belief and action in general. It belongs amoug the higher impulses yet to be discussed. The artist's intention expresses the permanence of this impulse and its exhaustive range over the material available to him. He selects his material under the law of voluntary interest. What constitutes the fitness of his material is the problem of what ideals are, and it is that aspect of the case to which we must now turn.

In the former connection we had to deal with the intellectual side only of constructive imagination, to answer the question: What constructions do we actually reach? We looked at art, etc., from the composer's point of view. There, while picturing the scientific and æsthetic process, we found no such things as ideals; only the intimation of them in the shape of vague distant ends toward which the imagination tends. Ideals are not mental constructions at all : if once constructed they would no longer be ideals: which only means that what we call ideals are emotional in their nature, expressing the drift or felt outcome of the constructive process, not any actual attainment of it. If my ideal man, for example, were an intellectual construction, I would be able to describe him.

Nature or Iaeals. ${ }^{1}$ What are ideals? What is art from the spectator's point of view? Evidently ideals are something felt in connection with present images; something, that is, in virtue of which peculiar feelings arise over and above the simple feelings of apprehension. In other words, conceptions of the kind produced under the lead of the constructive imagination have a peculiar quality, which leads us to pronounce them true, beautiful, or good. From the essential nature of conception we are able to reach, in a general way, the $\mathrm{s}$ within which this quality must be sought.

1 With this development, compare Hodgson, Theory of Practice, I. pp. 250-260. 
1. Conception proceeds by abstraction, and abstraction has been seen to be the mental tendency to pursue identities through the mazes of new experience. The gratification of this pursuit of identities arises as a feeling of pleasure whenever two elements of experience before disparate fall together in a unity or common meaning. Without such a process of identifying, with its accompanying gratification, no conception whatever can take place. One element of conceptual feeling, therefore, must arise from abstraction, and this element may be best characterized as the feeling of unity in a whole.

2. But an equally important, because opposite, aspect of conception, is generalization: the function whereby a concept gets application over a wider area of experience by a modification of its content. In abstraction I preserve my concept and neglect all experience which does not illustrate it; in generalization, I accept my experience and modify my concept to include it. It is a mental tendency away from identity to variety; and its gratification brings another element to conceptual feeling, i.e., the feeling of harmony of parts.

3. The intension or depth of a concept begets a phase of feeling in response to the peculiar qualitative or essential value of it in experience: while its extension excites only a feeling of its present accidental application. Man in intension excites in me the sympathetic and social feelings ; it indicates humanity, with the living thrill of interest the word suggests: but man in extension simply means men, anybody, everybody, commonplace and uninteresting. The emotion of intension let us call the feeling for meaning, a third essential ingredient in conceptual emotion.

By meaning we mean interesting quality, recognizing in the word all the springs of interest, intellectual, emotional, and volitional, already discussed. Our ideals are 
the things of most absorbing interest to us. Yet this interest is of a peculiarly objective character, as appears immediately below.

The extension-feeling scems to be no more than the simple logical emotion over again. It is the emotion which ordinary judgment and assertion arouse. "John is a man," is a judgment in extension : its relational quality is felt, but this feeling ceases, terminates there, and has no part in the feeling of ideals. If I simply mean that John is one of many men, knowing nothing more of John, then all is said : but when I say "John is a grand man," my word John has intension, depth, meaning, and I begin at once to look-or better, to feel-toward ideal humanity.

4. Further, conceptions are objective in their reference; they arise in the knowing function. They are guaranteed, as has been said, by the objective reality of the elementary presentations which they include. Further, this objectivity is not simply the presentative objectivity of passive imagination. It differs from it in two ways. In the first place, the concept is applicable to each object of the class it covers, while the product of imagination may be a new and unheard-of concoction of elements. And in the second place, the conception must be true in each of its applications to a consistent kind of reality-coefficient. The conception horse applies to all horses with sensational quality, the conception fiction to all objects which have only other than sensational or logical coefficients, etc. Consequently, two kinds of objectivity are felt to attach to ideals and to be involved in conceptual feeling: first, presentative objectivity to me (present also in imagination), and, second, the same coefficient of reality to every one else as to me (not found in imagination). Both these aspects may be covered by the phrase feeling of universality: a fourth ingredient in conceptual feeling.

Ideals, therefore, are the forms which we feel our conceptions would take if we were able to realize in them a sat- 
isfying degree of unity, harmony, significance, and unicersality. The first two properties we may call ideal form, the third, ideal meaning, and the fourth, ideal validity.

Feeling of Fitness. We are now able to give more exact definition to the state of consciousness before designated as feeling of fitness. It attaches to certain images of imagination which are available for conceptual construction : namely, to those which tend to take form in ideals. It indicates promise of progressive idealization under some or all of the rubrics pointed out above. But it precedes actual construction, since ideals are not positive constructions. If conception follows, then the feeling of fitness either becomes simple feeling of logical relation, or it attaches in turn to the new product as far as it is felt to be fit for further ideal construction. For example, I feel that each fact discovered in nature or the laboratory must fit in a construction of all similar facts called a law; but when this law, now a vague felt ideal, is itself discovered, then my feeling of fitness attaches to it only as it in turn serves as an element of a still broader ideal of systematic science.

Bain ${ }^{1}$ draws out a striking analogy between the process of constructive imagination in working up its material, and the process of muscular adjustment to external conditions, in which volition takes its rise. The two are essentially one in their selective and more or less tentative character, but they differ in their material, and in the rule under which they proceed. Muscular adaptation proceeds under the stress of practical necessity imposed by the environment : constructive imagination by a law of mental appreciation which does not reflect any arrangement or order realized in nature.

\section{§ 4. Conceptual Feeling as Intuition.}

In this feeling of fitness for conception, we have an advance guard, so to speak, of the apperceptive process

'Emotions and Will, pp. 374-77. 
itself. The reality or truth of conceptions we have seen to depend upon the reality of the particulars involved, and upon the validity of the laws of conception. Any validity, therefore, which attaches to general knowledge, as reached in conception-and there is no other way of reaching it-may be in a measure prophesied by conceptual feeling. If ideals are felt things, having their rise in vague analogies of relation, analogies which we find it impossible to interpret clearly in the regular categories of logical thought-then such feelings may often be the only guide to action and the safest criterion of truth.

By calling such feeling intuition, we simply. mean to say that it carries the reality-feeling by direct reference, and not by a constructive process. Conception does not carry belief, but acquires it from perception: but conceptual feeling often carries a belief-force which overwhelms the grounded conclusions of thought. It extends to all the varieties of conceptual feeling, finding place in art, in morals, in religion, in intellectual system.

Here is to be mentioned the strong intuitive faculty generally attributed to woman: a tendency to reach conclusions by "insight," as opposed to the reasoned and slower attainments of man. It is undoubtedly a feeling of relations, depending upon great liveliness in the sense of fituess. Women have ordinarily a lively imaginative endowment, and great delicacy of general sensibility in matters of fancy, taste, and fitness : gifts which artists share with them. And it is only what we would expect, that artists tend to show other feminine traits of character.

The place of sich intuition among the methods of truth is not in place here; but it may be said that there is no reason, in the nature of the case, that such feeling may not be misleading. The earliest feeling of reality has been found liable to the rude shocks which lead on to well-grounded belief : so this feeling for truth may vary in vilue from the 
brilliant forecasts of an Agassiz or a Cuvier to the vacillating presentiments of a hysterical girl. ${ }^{1}$ Such error resides, however, in the content, the particular fact, of intuition.

\section{§5. Range and Kinds of Conceptual - Feeling.}

The various ideals to which we find ourselves committed with greater or less emotion may be classed under three heads, according to the classes of data which are felt to be fit. The tendency to gratification in the more logical application of the principles of unity and variety is felt in an ideal of system : what we may call feelings of the systematization of truth. The principles of progressive ideal conception in their application to the elements of personality lead us to ideals of character, feelings for the good, or ethical feelings. A further outgo of conception in its felt grasp upon all the more particular aspects of its content, upon all possible objects of feeling, gives us ideals of beauty: the cesthetic feelings. These may be considered in turn.

\section{§6. Feeling for System in Mental Construction.}

Scientific system. The exercise of the scientific imagination is accompanied by the scientific ideal, and its materials are selected as fit to realize this ideal. Of all conceptual ideals, the scientific is most plain. Here the criteria of unity and variety have almost exclusive voice, and apply throughout all the kinds of relation which arise in the apperceptive process. The ideal is complete unity of conception in the infinite variety of objective fact, and each new generalization in any science, as chemistry, biology, psychology, is in so far gratifying as a partial realization of it. The conceptual

'See Lotze's interesting discussion of the intellectual worth of feeling; Microcosmus, bk. II. chap. v. As he says, the "world of values" (felt) must be explained by the "world of forms" (cognized), ibid., p. 247. 
feeling, however, antedates the actual construction, and hence its presence in strength, if supported by the other elements of constructive imagination, marks the scientific genius. The pleasure derived from the contemplation of systematic truth, as opposed to its anticipation, is a different feeling, i.e., simple relational feeling. Yet this latter is seldom unmixed with a further feeling of meaning or fitness for yet new advance.

Philosophical system. The same tendency of thought. urges us on to the construction of a system of philosophy in which all the partial truths of the various sciences, all the facts which are not accredited to particular sciences-indeed all the phases of reality under whatever aspect they may be viewed-are held in a single constructive effort of the mind. This is a philosophy : absolute unity of ground and infinite variety of application: the resolution of all half-truths in one whole truth, the universe. No philosophy can completely satisfy the constructive ideal which does notreach a single all-comprehensive principle.

\section{§7. Ethical Feeling.}

Its Coeffleient. Premising the conceptual nature of the moral feelings, we may ask after their peculiarities. What is their general nature, and to what kind of experiences do they attach? Using the words good and bad, to express what we mean by moral approval and disapproval, we may examine consciousness to find their legitimate application. The moral coefficient is that in experience which leads us to attach to it the predicates good and bad: it may be called, for the present, moral quality.

Moral Quality. A rough generalization easily leads to the conclusion that good and bad, in their moral sig- 
nificance, attach only to living agents. If I say a man has a bad character, I mean that he is capable of bad conduct. If I say a knife is good, I mean simply that it is useful : not that it is moral at all. But not all actions are moral. Some actions are forced. I may be driven to perform an act against my will. This is not moral. So we reach a further point, i.e., moral actions must be voluntary acts, or acts of will, whatever will may turn out to be. Further, not all voluntary actions are moral. I may dine at two o'clock or at six; I may take my walk north or south: these actions are morally indifferent. What further peculiarity attaches to some acts of will, whereby we call them good or bad?

A reference to the general psychology of conceptual feeling, as already developed, will throw light upon this point. We found the feeling for ideals to involve, in its object, harmony, universality, and meaning: so, if the moral feelings are rightly classed as conceptual, only those states of will which fulfil these conditions in some degree will be found to excite moral approval or disapproval.

I. Moral Quality as Harmony. Acts of will which are moral can never be taken out of their environment in consciousness and conduct, and pronounced good or bad. Moral actions are those which are harmonious with each other in reference to an ideal. A morally indifferent act is an act which stands alone, which is of no value to anybody except the doer, and of no value in the conceptual complex of the doer's progressive agency. The reason that my dinner-hour is indifferent is that it has no value to any one but myself, and none to myself except my convenience. As soon as it does become a matter of health to me, or comfort to any one else, i.e., gets a setting of relations more or less conscious, it does become moral. Moral quality, therefore, attaches to an 
act of will considered as an element in a complex of interests, my own and those of others. Moral predicates attach to certain felt possibilities of conduct considered in relation to all other possibilities of conduct.

It is this aspect of the case that men have in view in locating morality in character. ${ }^{1}$ A good man is not a man of particular good acts only, but of general prevailing good acts, of good character. This means that his acts of will are so harmoniously good that they bespeak a disposition or permanent attitude of will. What it is that this harmony refers to as the ideal good is a matter of further inquiry.

II. Moral Quality as Universality. The universality of ethical feeling arises in consciousness in two new and distiuct forms. Not only is morality objective in the sense that others are held by me to the judgments that I myself make; the universality of truth in general: but the existence and claims of others enter as factors in the content of the feeling for myself. The feeling of sympathy is one of the elements whose satisfaction this moral satisfaction as a whole must include. And further, simple disinterestedness, as all conceptual feeling involves it-value apart from gain or loss to myself-does not here suffice; but the feeling of restraint, constraint, obligation, takes its place. These two factors may be considered further.

A. Moral Sympathy. Moral sympathy attaches exclusively to the idea of persons, and carries with it the notion of self. The simple idea of suffering which was found sufficient for sympathy as an expressive emotion, now gains its full personal reference. This feeling may be described as the consciousness of the equality of 38,39 .

'Cf. Hodgson's admirable exposition, Theory of Practice, II, pp. 
individuals in reference to ideal good. The conceptual construction which ethical feeling anticipates, proceeds out from the postulate of a plurality of individual wills acting in harmony. The ethical feeling is based on the social feeling and grows in generality with it.

The question of altruism vs. egoism already stated receives here its definitive answer. Ethical feeling cannot be based simply on egoistic feeling, for the reason that the ethical ideal involves more than individual interests. It is a generalization away from the individual agent, and by its very conception demands equally the consideration of the interests of others. Even though we should find that simple sympathy arose psychologically as an expansion of personal pleasure and pain, still this would not begin to explain the universality of the ethical consciousness; simply because the construction of such egoistic factors results in a concept which does not arouse moral emotion.

B. Moral Authority : Feeling of Obligation. ${ }^{1}$ The second aspect of moral universality is the feeling of obligation, or of subjection to moral authority. As already said, it is a consciousness of both restraint and constraint. It is further felt to be from within, i.e., not to have any assignable cause outside of consciousness. It restrains from one course of conduct and constrains to another. It does not enter simply as a possible alternative which I may or may not embrace, which may be neglected or not as I please; but it has an additional element of feeling, the feeling covered by the word ought. I may go to a lecture or not; I ought to help my poor neighbor. This is ordinarily called the imperative aspect of ethical feeling.

Ethical authority may be distinguished from several other kinds of authority in consciousness.

1. It is not simply the authority of truth over belief: it differs from conviction in that it attaches exclusively,

${ }^{1} \mathrm{Cf}$. what is'said on the feeling of responsibility, below, Chap. XVI. §4. 
as has been seen, to states of will. The laws of logic are not the laws of ethics.

2. It is not the authority of fixed ideas, whether they be ideas of theory or of action. For a fixed or controlling idea does not admit the choice which is felt to constitute an act of will. The same may be said of acts performed under overwhelming emotion. The feeling called freedom, which attaches to all acts of will, is absent-whatever, again, that may turn out to be.

3. It is not authority extraneous to the will itself. Later analysis will show that nothing can influence the will that does not act as an antecedent condition to choice, as motive. Moral authority, therefore, must enter somewhere in the complex conditions which issue in an act of will.

For these reasons, ethical systems agree in linking obligation with freedom. We have only to do with the feelings of obligation and freedom respectively, not with their ethical or metaphysical worth; and it may be well at the outset to emphasize the point that in the following pages the only problem attempted is an analysis of the subjective moral consciousness.

In view of these distinctions, moral authority is seen to be the feeling that a peculiar worth attaches to cer tain motives or ends in relation to other motives or ends. This worth is further not merely a recognized worth in view of an ideal, but a worth felt to be imperative upon my free choice. In other words, moral authority may be defined, at the present stage of our inquiry, as a feeling of an imperative to the will to the free choice of $a$ moral end. The nature of the moral end or ideal is reserved for later remark.

Upon this determination certain remarks may be ventured. First, the imperative of the feeling of obligation is an unconditional imperative. While it is true that it arises only in connection with alteruative courses 
of action, yet when once arisen it is, as an ought-feeling, quite independent of such connections and conditions. This Kant has emphasized by the phrase "categorical imperative." Second, the feeling of freedom before alternatives is still present, even when the moral imperative is clearly attached to one of them. Though I feel that I ought to pursue a certain course, still I feel free to disregard my own moral injunction and pursue a different course. Third, that the ought-feeling is always relative to an ideal, is seen in the fact that the same course of conduct is at one time right, at another wrong, or indifferent. The morality, therefore, as already said, covers the harmony of all possibilities with reference to an ideal. And, fourth, moral feeling always attaches to the concrete, to particular acts of will. We have no general feeling of right or wrong. We may vainly attempt to depict the moral ideal as an abstract ideal, and through it to arrive at the sense of right in the abstract: but moral decisions, as such, are always decisions on actual concrete possibilities. Moral authority, therefore, is the form of all ethical feeling: but ethical feeling is more than form, since the content of an ethical decision, the concrete course enjoined, may vary even to contradiction.

Ground of Moral Authority. The further question, therefore, arises: How can a formal principle of the activity of will get its application to concrete courses of conduct? Why are not all acts of will included in this form, i.e., why are they not all moral? How can a regulative principle of will get to be a criterion of conduct? Several possible answers may be suggested. It may be said :

1. There is a conscious ethical ideal or end with which possible alternatives of conduct are directly compared. This is disproved by the fact that we are not 
conscious either of such an end or of comparing our alteruatives with it. There is an ideal, but we cannot conceive it-a point made plainer below.

2. It may be said that there is a simple reaction of feeling, perhaps biological attraction or repulsion, toward or from actions. This will not do, for the question would still remain: Why, then, are such reactions felt to be imperative? Why do some attractions and repulsions have the ought-feeling, while others do not? This theory simply denies moral authority as a separate kind of experience.

3. We may be told that we have a direct cognition of moral quality in actions ; and wherever thus cognized, it is felt as imperative. This will not answer, since the same acts, qua acts, may be, at different times, right, wrong, or indifferent: and to inquire into the ground of this difference is to admit some criterion over and above any quality the individual act may have.

4. Ruling out these answers as inadequate, there is only one alternative remaining, namely, that the determination of conduct in the concrete as morally imperative takes place by a reaction of consciousness upon $a$ group of alternatives in such a way that these alternatives are arranged in a scale of values with reference to the moral ideal and to one another, the highest value being approved as relatively right, and the others disapproved as relatively wrong.

In this position, it is seen, the determination of the imperative is a relative determination; a determination of the adjustment of particular alteruatives to each other as regards worth for an ideal. In other words, the particulars are the material of different degrees of fitness for a generalization. That generalization-could we make it-would be the moral ideal, and the peculiar feeling of approval or disapproval of the most fit in 
possible conduct carries with it also the feeling of oughtness.

It may be said, in regard to such a solution, that it leaves the main question unanswered, the question as to how a formal principle of oughtness gets application : that it does no good to widen the reference of the imperative to a group of particulars which have moral worth relatively to each other. But this objection holds only if certain such groups have moral predicates while other groups have not-as we found certain courses of conduct indifferent while others are not. Now this is not true. No such groups of alteruatives are morally indifferent. Indifference here means relative rightness. My decision to dine at six o'clock is indifferent as a single act, but it is not indifferent as a relative act. Relatively, I do right in dining at six, as I would also do right in dining at two. In the relations of my conduct to any interests, there is only one act, dining, and it is indifferent; as soon as any other interests become alternative to my dining at two or six, my dining is a moral act. The question, why it is that certain alternatives take rank as morally fitter than others, belongs under the wider question of the nature of the moral ideal.

The conclusion on moral authority is, therefore, with Butler and Sidgwick, that it is psychologically "ultimate and unanalyzable." Why there should be an ought-feeling in connection with states of will is as inexplicable as the states of will themselves are. Both the elements of ethical universality mentioned are recognized by Sidgwick, ${ }^{1}$ who uses the word objectivity to express the essential presentativeness of moral distinctions. The term "conceptual" answers the purpose as well, and at the same time suggests the ideal reference of oughtness, to which Sidgwick does distinct injustice. This objective or universal value of moral feeling is emphasized under the phrase "practical reason" by idealistic thinkers, and under that of right anu wrong "cognitions" or " judg" ments" by intuitionists.

Conclusion on Moral Coefficient. In regard to the subjective side of moral quality-the conscious feeling of the presence of the right or wrong-we are now able to speak more definitely. Negatively it may be said :

${ }^{1}$ Methods of Ethics, 4th ed., chap. III. Cf. above, Nature of Ideals.

${ }^{2}$ Calderwood, Handbook of Moral Philosophy, chap. II. 
1. Moral quality does not attach to single pictured alternatives or ends as such. An end is nothing apart from the will whose end it is; it is an end only as it is set up by an agent. To the artisan, a penknife is an end for construction. We cannot say that moral quality attaches to the penknife, for it does not exist; nor to the conception of the penknife, for it may be either right or wrong for him to make a penknife. The moral judgment does not arise till we know more about the case: the relation of this end to other ends he might pursue, and his own relation to other men.

2. It does not attach to motives. The ordinary phrase "good or bad motives" is misleading and inexact. For a motive is simply a moving influence and may be either a conscious pictured alternative, an end, or a vague affective or organic motor influence, an affect. The former has already been ruled out. The latter does not answer the general criterion of moral conduct, that it be the outcome of free choice. Such affects are seen in impulse, in animal instinct, in insanity; conditions to which no moral worth is attached.

3. It does not attach to actions; actual volitions as such; for they are right, wrong, or indifferent. We say we must know why a man did so and so before we pass judgment upon him.

More affirmatively, therefore, we may conclude, in conformity with the determination already reached, that the moral coefficient is the feeling of an attitude of the will toward or from one of alternative courses of conduct as relatively fit or unfit for construction in a moral ideal. And this fitness is, as far as can be discovered : first, the degree in which a course of conduct is felt to harmonize with most interests, to be approved by others as well as by myself, and to be imperative, though not executive, upon my choice.

The moral coefficient is thus seen to have two sides, a 
subjective and an objective side. Subjectively it is an approving attitude of will with felt obligation, all that is meant by the word ought: objectively, it is harmony and universality, what is meant by the word right. About the ought, the above is all that we have to say; it is an ultimate category of feeling, whatever its origin may have been. As to the right, certain rules of conduct are found below, which find their highest expression in the Christian principle of love, the Golden Rule. It embodies the moral ideal, the next topic of treatment, as far as the ideal is a matter of conception.

III. Moral Ideal: the Ethical End. Of the elements found necessary to ideals generally, that is, necessary to conceptual feeling, meaning was included no less than harmony and universality. Having now looked at the elements of harmony and universality involved in ethical feeling, it remains to consider the element of meaning. To draw again a distinction already made, not the spectator's point of view alone must be consulted, but the composer's, the constructing agents; in this case, the doer's point of view. If I would do right, what kind of a pattern or end do I set myself ?

Notion of End. In the treatment of the motor aspects of ideal feeling below, the conception of end becomes familiar; yet here it is necessary to point out and emphasize certain distinctions. An end is that which I consciously present to myself for possible pursuit. It must be clearly distinguished from motives, ${ }^{1}$ which are any influences whatever that may come to bear on the will, whether they be consciously presented or not. Only some motives are ends. Further, an end does not always carry

1 This distinction is shown by Sidgwick (loc. cit., pp. 38, 39), follow. ing Butler, to be a necessary one, whatever our ethical theory may be. Even the hedonist (see below) makes some distinction between impulse and more or less reasonable purpose. 
the presentation of self; a child has an end when it imitates the movements of its nurse, before it gives evidence of reflection upon its own mental states. Consequently there may be a great many ends in consciousness at once; which means that the end is distinct from volition. Volition is the choice of a particular end.

Subjective vs. Objective Ends. ${ }^{3}$ In saying that an end must be consciously presented, it is further meant to exclude organic and biological results which seem to us to be due to presentation or purpose. The physical organism is full of adaptations all supposed to minister to the greatest pleasure and to produce the least pain. Yet pleasure and pain are not necessarily the ends of our voluntary physical activities. In order to become subjective ends, they must be pictured as the objects of the voluntary process; otherwise being organic, they are a form of objective end.

Equally objective are the speculative ends which philosophers find in nature as a whole; design in nature or teleological ends: immanent development of an absolute principle in nature; ends of self-realization. The psychology of subjective ends has nothing to do with such determinations.

This distinction cannot be too carefully weighed, because of the wanton violation of it in certain ethical discussions. The child reaching for its food-bottle may not picturo the pleasure, and it may not picture itself; it may picture only the bottle. 'The organic end is the gratification of appetite, and this results in pleasure ; the cosmic end may be equally present; but the conscious end may terminate short of both of them. The violation of this distinction is a case of the " psychologist's fallacy." We adults have learned that the organism is an adaptive thing; that nature has ends; that the fittest for these ends survive: and so we read into the childconsciousness the intentions which we think must be those of

'Question of subjective end vs. question of Summum Bonum, cf. Sidgwick, Methods of Ethics, 4th ed., p. 3. 
nature. Even though it were granted that all voluntary action arose and survived by exclusive reference to pleasure or to self-realization, yet it would be a patent fallacy to say that the only voluntary end is either of them-that consciousness has all along been versed in our biology or our speculative ethics, and has aimed to fulfil the one or the other. Consciousness has no inkling of the $\delta v^{\prime} v \alpha \mu z$ of Aristotle, or the conatus of Spinoza, or the trieb of Wundt and Schneider; of the "strife for existence" of Spencer, the theoretic "reverence for law" of Kant, the "self-realization" of Green, or the dialectical " becoming" of Hegel. Let us discover these things if we may, but do not let us say that a man is not moral unless he have a realizing sense of them.

Moral vs. Natural Ends. A further distinction is possible between ends which are accompanied by moral feeling and ends which are not. The latter are important for the psychology of volition; they are the indifferent ends already spoken of, and may be designated natural. Only moral ends concern us in the theory of ethical emotion.

Theories of Moral End. The question of the moral ideal, then, becomes this : is there a subjective moral end called the "good," which the right-doer sets before him; and if so, what is it? A variety of answers have been proposed in the history of ethics. Considered psychologically, we may classify them as affirmative and negative answers, i.e., those which attempt to justify a particular conception of the moral ideal, and those which do not.

I. Affirmative Theories: 1. Happiness Theories. A general class of theories affirm that happiness is the eth. ical end. The happiness theory is denominated Eudaimonism or Hedonism. ' In favor of a happiness theory of the moral ideal, several lines of argument are historical, each upheld by a school of ethical thought.

${ }^{1}$ The difference between these two words is about that between the

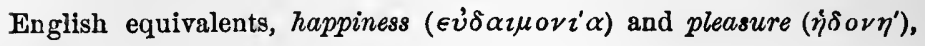
or higher and lower enjoyment. 
Arguments. a. We are told that happiness is the most natural and spontaneous object of pursuit; that, while all other ends have to be justified to consciousness, happiness needs no such justification. It is an implanted principle, the love of pleasure ; and volition is only following the example set by organic nature in pursuing happiness. So the ethics of Naturalism.

b. Again, it is contended that happiness and well-being go hand in hand. Happy states are healthy states, and this holds of will as well as of all other states of consciousness. The law of life and development, therefore, is the law of happiness: so Egoistic Hedonism. Further, our intellectual life is a progressive developing principle ; its implicit end is self-realization ; and self-realization comes by the gratification of the higher nature of self, by happiness. So the Eudaimonism of Subjective Idealism.

c. Yet again, what is true of the individual holds $a$ fortiori of the race. Men are social by nature. Society develops by the greatest happiness of the greatest number. Happiness is the general end of humanity. So Universalistic Hedonism. And this union of individuals is possible only by reason of an universal consciousness which is realizing itself through us. Happiness, gratification, is the law not only of self-realization, but of the realization of absolute reason. So the Eudaimonism of Absolute Idealism.

Reply. In reply to these positions, in the outcome of which empirical naturalism and speculative idealism are in unusual alliance, it may be said :

a. If happiness be individual egoistic happiness, it comes in conflict with the happiness of others. ${ }^{1}$ Nature,

${ }^{1}$ This position is adverted to below under the headings Desire and Motive. For the best examination of the egoistic basis of hedonism, see Sidgwick, loc. cit., bk. I. chap. IV, also bk. II. 
then, simply affords a theatre for warring interests, and so does consciousness, unless we deny the sympathetic feelings altogether. This may be true of organic ends in nature, but it satisfies neither the moral nor the philosophic consciousness.

b. If happiness be the happiness of the greatest number (universalistic), it is either consistent with individual happiness or it is not. If it be consistent with individual happiness, then we go back to egoistic hedonism to get our criterion; if not, then we have no means of knowing what actions of individuals will conduce to general happiness, i.e., we lack an end altogether. General happiness may be the organic, natural, objective end; but as subjective end its only formula is an analogy from individual happiness.

The appeal of Mill" to sympathy as the "ultimate sanction of the greatest happiness morality" is, therefore, vain; for though we may find in sympathy the means of bringing the interests of the community into the consciousness of the individual, we still lack means of interpreting those interests apart from the conscious experience of the individual as felicific or otherwise.

c. The moral imperative often comes into direct conflict with the natural pursuit of happiness; it seems to enjoin the subordination of personal pleasure to the happiness of others. Such a conflict would be impossible if personal happiness were the subjective end.

$d$. No help is reached by a qualitative distinction between lower and higher happiness (qualitative eudaimonism ${ }^{2}$ ), higher happiness being the highest good. For how can we distinguish happiness as higher and lower? Only by some criterion or standard of reference back of happiness.

${ }^{1}$ J. S. Mill, Utilitarianism, chap. III.

J. S. Mill. 
e. We cannot accept gratification with self-realization as the subjective end; for if we really pursue gratifica. tion, it is an end and not a means, and we fall back into egoistic hedonism. But if we pursue self-realization, we have no right to interpret it exclusively in terms of gratification; indeed, our moral approval attaches consistently to acts of self-denial which bring no evident gratification, except the gratification of the moral impulse, to auybody. If we endeavor to hold the two notions together, with Green, and make the end gratification as tending to self-realization, then we distinguish kinds of gratification qualitatively again with Mill. How do we know what gratifications tend to realize self? The same is the result if we make moral gratification the only legitimate gratification.

2. Utility Theories. A second class of theories make the ethical ideal some form of the useful. Right conduct is always useful, and useful conduct is always relatively right. Historic Utilitarianism has taken one of three forms : it may become a broader statement of eudaimonism, by construing the useful as that kind of conduct which brings happiness to somebody; or it may stand for self-realization, by construing the useful as that which is beneficial, whether it be pleasant or not; or, finally, it may refuse to construe the term useful in any exclusive way, meaning the useful in general-either the detached and accidentally useful, or the coördinated and cosmically useful.

Criticism. 1. On Utilitarian Hedonism, the above general criticisms of hedonism hold. If I pursue the useful in order to get the pleasant, my end is, after all, the pleasant.

2. As regards utility as self-realization the question of a criterion at once arises. Happiness will not do, as we have seen, as a criterion of conduct which realizes self. 
Character or virtue as an end for self to realize sounds plausible, but what kind of character? -and what acts tend to virtue? The only answer is, satisfy your conscience; but I could do this, and would as well, if I knew nothing about the law of self-realization. My principle has accordingly given me no information, and I have to resort to experience for concrete decisions, as before.

There is here, however, the case of those who make truth to conscience their end, i.e., absolute fidelity to what light one has :

"To thine own self be true

And thou hast done with fears,"

-as Swinburne and Emerson are fond of telling us. But this "conscientiousness" asserts an ideal of execution-an attitude toward the right-rather than the determination of what is right. As Sidgwick points out, the fanatic is no more right because he is cock-sure of it, and so over-scrupulous about observing it.

3. On utility considered as cosmic progress, it may be said that it gives no practical criterion of conduct, unless there be in the individual some response of a peculiar kind to the progress in which he shares ; but to consider ethical feeling such a response, while perhaps philosophical in itself, is worthless for the psychology of moral ends. For the question then is : What particular form in consciousness does universal progress assume, what presented form, as object of pursuit? What concrete action is the conscious equivalent of cosmic progress? The other alternative, that the moral is the accidentally useful, is only another statement of the doctrine of naturalism. Our moral nature does not approve what is accidentally useful in conduct.

The Evolution Ethics belongs in one or other of these categories. Either evolution is teleological, an end-process, or it is a'process of growth by happy accidents. If it is teleological, 
and moral feeling is the conscious reflection of this progress, then either natural biological ends state the whole case, or cosmic progress itself is the end. But the first clashes with moral feeling, and the second gives it no criterion. And if evolution is not teleological, concrete utility or egoistic hedonism should satisfy the moral consciousness. Further, it is difficult to see how inherited experiences of utility, as claimed by Spencer, would help the case. For even if it be granted that the feeling of oughtness has arisen from race-experiences of utility, still in the individual consciousness there is no knowledge of it ; it is only the philosopher who sees in all his moral judgments a higher utility. 'The subjective end, the pictured end, may be the hurtful, and the fact is not altered whatever derivation we may give to it. There can be no doubt that utility is applicable to all of nature's ends, but it is pretty evident that I do not share all of nature's ends, and that I have some little ends which I can find no evidence that she shares. It is probable that the economy of nature includes my morality ; but yet I cannot accept the c onomy of nature, as I understand it, as doing justice to my moral needs. 'To repeat an earlier warning, we must not confuse extraneous ends, either organic or speculative, with the conscious subjective end. It is the fundamental confusion of such opposed thinkers as Spencer and Caird.

General Criticisms of Hedonism and Utilitarianism.'a. A further criticism of both these classes of theories may be drawn from the fact that they rest the case upon experience. It is difficult to see how experiences either of happiness or utility could give rise to the universalitythe imperative character-which we have found attaching to ethical feeling. Why do I hold other men amenable to my decisions of right and wrong? Their pleasure and profit might be different from mine.

$b$. If moral feeling results from a series of consistent pleasurable or useful actions, why do not all such series of consistent pleasurable or useful actions become moral? Suppose all my ancestors had found it pleasant and useful to dine at six, and I myself had likewise, it

1 For detailed criticism of the view that pleasure is the object of all desire, see the section on Desire, below, Chap. XIV. $\$ 2$. 
would not on that account be wrong for me to sacrifice: the advantage and dine at two.

This is the same difficulty we have found attaching to empirical derivations of space and time. If any series whatever of intensive sensational data get arranged in space and time order, all such series ought to ; but they do not. So if moral feeling results from pleasure and pain series, all pleasure and pain series ought to have some show of morality about them ; but they have not.

Mill $^{1}$ endeavors to meet this objection to Bentham's utilitarianism by holding that such experience begets "habits," which habits are, like Hume's " custom," a "new state of feeling," i.e., ought. In other words, I no longer need to. prove the act useful and thence judge it right ; I have come. to $\mathrm{feel}$ it right. Spencer attempts the same by his resort to race experience. But this only means that for my acts, the ought-feeling, and not the useful results, is my criterion. The ought-feeling may have arisen in the progress of evolution, as far as this point is concerned, because it is a useful feeling; but the law of survival is sufficient to prove it to be clearly differentiated from the atility-feeling. As Hodgson ${ }^{2}$ shows, the utility theory can only be a theory of ethical judgment, not of the immediate feeling, which oughtness is.

c. Both these theories fail to recognize the spontaneity of moral emotion. They introduce calculation and discussion into conduct. Conscious moral decisions, however, generally lose in clearness and certainty if we weigh the pros and cons. In matters of conduct, first: thoughts are usually best thoughts.

$d$. It is difficult to give any meaning to the feelings of responsibility, sense of wrong-doing, and remorse, on either of these theories. If a man acts for happiness or utility and does not get it, he has simply made an error of judgment. Regret or sorrow would be the only emotion experienced.s

1 Utilitarianism, chap. II.

2 Loc. cit., Ir. p. 46.

${ }^{3}$ Cf. Green, Introduction to Hume, vol. II. pp. 13-16. 
3. Formal or Kantian Theory. According to Kant, the moral law is the ethical end ; right conduct is conduct performed from reverence for the moral law. Morality enjoins such acts as tend to the universal observance of law. Upon this theory the following points may be made.

Criticism. a. It requires a statement or definition of the moral law as a subjective standard of reference for conduct. Such a statement or definition must take the form either of generalization from concrete cases of felt imperative oughtness, or of a command upon the will from some extraneous source. The latter we have seen to be impossible : the will can be influenced only through motives. And a generalization from concrete cases of obligation we have seen to be equally illusory. The imperative proceeds by a new relative adjustment of alternatives for every act of choice; not by a comparison of each alternative with an abstract statement or law. This difficulty is such an evident and practical one that every moralist encounters it in endeavoring to draw an adequate general formula of duty.

$b$. Admitting the formal imperative as the law of will, the question of its possible application has already been discussed. We have found such an application possible only on the ground of an attitude of will toward its present alternatives as relatively more or less fit for ideal construction. What is universal is the form-the imperative feeling; not the content-the criterion. The content is particular in the first place and remains so in the last place, and the attempt to give it universal statement as law is speculative and foreign to the spontaneous ethical consciousness.

c. The last criticism is practically admitted by Kant in his attempt to state the law, i.e., "So act that the maxim of thy conduct may be fit for universal accept- 
ance." In other words, act with an eye on the consequences.

d. As the experiential theories, so the formal theory opens the door for deliberation and destroys the spontaneity of moral feeling.

II. Negative Theories of the Ethical Ideal. A class of ethical theories, further, assert that there is no single adequate statement of the ethicalideal or end. Of these the following are typical.

1. Intuitional Theories. Some intuitive or "commonsense" thinkers hold that we have no single absolute conscious standard of moral feeling, but that we have immediate or intuitive cognition of moral quality in acts. Such intuitions, although given on occasion of concrete choices, nevertheless have the value of absolute laws of conduct. The moral standing of a given alternative of action is ascertained by the application to it of one of these moral intuitions.

Criticism. In spite of many excellences which will suggest themselves, this theory is inadequate for the reasons below.

a. We have failed to find the stable moral quality in acts which such intuitions require.

b. The facts do not sustain the universality claimed for such intuitions; the laws of veracity, homicide, etc., are not universal, but relative. Hume is right here. It does not do to say that conscience has been perverted, and hence the disagreements between races and individuals ; for to admit that some are depraved is to deny the only consensus possible to prove their uniformity. We must take conscience as we find it, not as we wish it were, in dealing with the psychology of man as he is.

c. These intuitions are singly inadequate, as is seen in their possible conflicts, as between justice and mercy 
and in their historical development. The condemnation of polygamy and slavery is an historical growth.

2. Relative Theories. Relative theories have in common the denial of the universality of the ethical content or ideal ; while they may or may not admit the universality of the formal imperative or feeling of oughtness. In regard to the latter question, the foregoing discussion has been sufficiently explicit; our conclusion was that an unconditional, absolute, and imperative feeling of universality attaches to our distinctions between right and wrong. It remains to show that, while the form is thus universal, the content is not. My ethical consciousness tells me universally that I ought to do right, but it does not tell me universally what I ought to do, to do right. In every dilemma I may be in, it is a question as to what, which I ought to choose; not whether I ought, after I have chosen.

Conclusion on Ethical Ideal as End. If what has been said about moral quality and authority be true, the doctrine of end is plain. The content is given in concrete acts; it is a relative content. The form is given as an universal imperative. The form cannot be end; that would be tautology, i.e., I ought to do what I ought. But an adequate content as universal end demauds a perfect generalization of possible concrete choices, which is impossible. Hence there is no universal subjective end.

The examination of the foregoing theories has made it clear that the traditional statements of the schools are inadequate. And it follows from a sufficient understanding of the nature of conceptual feeling, that all statements of the ethical ideal must be inadequate. Fitness for an end cannot mean adequate embodiment of that end; no one's alternatives of conduct can cover the whole 
of the possible fields of adjustment of wills to one another in a developing social organism. The ethical ideal, therefore, as far as it is conscious, is the degree of harmony and universality in conduct which I find my emotional nature responding to with imperative urgency. As an ideal, it is relative and changing in the life of the individual and of the race; yet that embodiment of it to which the individual or the race at any time responds is of absolute and unequivocal validity then and there.

The highest embodiment of the ethical ideal is the conception of the character of God. This does not give a statement of the ethical ideal, however, for the conception of God as a perfect being is of a character which realizes our moral predicates to perfection, and as such shifts with our development and that of the race. Instead of the end consisting in our conception of God's character, the reverse is true. God's character to us results from our conception of the moral end.

Rules of Conduct. There are, therefore, valid rules of conduct which are imperative upon the individual, not because they are universal statements of the ideal, but because they generalize our concrete intuitions of the right. They are the objective side, mentioned above, of the moral coefficient. The worth of each of them, however, in any case, depends upon its support from the moral consciousness in that particular case. Such principles are the so-called laws of the practical moralist : veracity, temperance, prudence, mercy, forgiveness, etc. These rules are absolutely binding wherever the moral consciousness gives them an application; but they are not applied by the moral consciousness universally. For instance, veracity is sometimes subordinated to a higher demand of ethical feeling, such as loyalty, humanity, or charity. 
Such exceptions are rare, however, in homogeneous society, and it is a question whether the objective demand for uniformity does not become in most cases a balancing motive for the maintenance of common standards: there is a tendency of our moral nature to rest satisfied with convention. Yet the possibility of transcending these gencral rules of content is always open, and the denial of moral progress is the price of its denial. Moral progress, like intellectual progress, is a matter of broader conceptual outlook upon conditions of greater social, as well as personal, complexity; and there is no reason in the psychology of the case that there should not be progressive moral insight-broader statements of the ethical conscionsness at different epochs-and broader consciousness of the need of such statements in individuals. Indeed, it must be so, and this is only to say from the point of view of psychology what we are told unequivocally from the point of view of historical study.

The spontaueous character of ethical feeling brings to prominence what was said above of conceptual fecling as intuition. Intuition here is felt conviction, anticipation of truth by vague analogies of relation, which is not itself understood. In matters of right and wrong, as in matters of rsthetic fecling, such intuition is the only guide we have. In the anticipation of logical truth, it may be supported or revised by conscious reasoning; but in ethical and æsthetic matters only the intuition of another can overcome or justify it. If women served on juries, criminals would have a better chance. 'The broadest and truest statement of the ethical law is the Christian law of Love; but an important element of its truth is that it admits of progressive interpretation.

In actual life our choices rest usually between two rules which contain all the rest, i.e., justice and sympathy, both of which we feel must tend to happiness.' Sympathy is right; it is an essential element in the moral coefficient; it is the individual side of social feeling. And justice is right; it is the general disinterested side of social feeling. As to which prevails, that depends upon the character, as emotional or stern, not upon any rational solution. One often feels that it is a question of a right and a more right; the relativity of the decision seems to work up into consciousness.

Moral Judgments. It is in the conscious application of these rules of conduct to particular actions that the ethical emotions take on the form of judgment, i.e., "this act of

'See Hodgson's fine discussion, loc. cit., p. 40, and context. 
veracity is right," or, generally, "veracity is right." Such judgment, like all judgment, is the assertion of the relations implicit in conception. Ethical judgment is, therefore, the belief form of the ethical ideal as far as its elements are conceived. Just as belief in external reality takes the form of judgment, or assertion of the sensational coefficient; and belief in logical reality, that of the logical coefficient ; so ethical judgment is the assertion of belief in the presence of the ethical coefficient or moral quality. Both moral and rsthetic feelings are anticipative, while the corresponding judgments are more or less retrospective.

Psychologically, the clearest exhibition of moral judgment is felt after moral hesitation and indecision. The process of synthesis of motives, called below "volitional apperception," results in a moral assertion or judgment which represents, not one of the motives alone, but that motive in the light of, and as related to, all the others. What we have called "intention," therefore, comes to apply, as Sidgwick says, "to "all the consequences of an act which are foreseen as certain and probable;" as distinguished from motive, which applies only to the desired consequences. For example, I decide to go gunning on Sunday morning rather than go to church. I feel not only reponsible for my motive, the getting of sport on Sunday, but also for the injustice I have done in rejecting the opportunity for spiritual improve ment, i.e., for my intention.

Conscience. In the word conscience, the ethical consciousness has its broadest characterization. Conscience may mean and does mean three very distinct things; three things, however, so essentially one as a mental fact that the use of a single word to cover them has its full justification. If we cut the mental life right through at the moment of positive ethical feeling, getting a section of the mental stream, so to speak, showing all that is felt at that moment, this section is conscience. The three

Below, Chap. XVI. $\$ 1$.

'Loc, cit., 4th ed., p. 202. 
portions of the section correspond to the three determinations we have already made, i.e., moral quality, moral authority, and moral ideal.

Let us take a concrete case of action from conscience: I give money to a beggar because I am bound by conscience to do so. The moral quality of my act is my feeling of its harmony with my better acts as a whole, and the exaction I make upon other men to be charitable also ; without this, conscience would be wanting - the act would be indifferent. The moral authority of the act is the feeling which at once arises that this quality has an immediate reference to my will. I am bound to choose it as my act; without this, there is no conscience-conscience is dead. The moral ideal is the outreach of my feeling toward a state of will in which such a relative and hesitating decision would yield to clearer and more direct moral vision; a state of will which I cannot picture, cannot conceive, but which I feel my will is meant for, and for which I feel my present act for conscience sake is the only means to prepare me.

Consequently, from the point of view of the individual consciousness, conscience is a spontaneous reaction of approval or disapproval of one of alternative ends, as of higher relative excellence with reference to an ideal unseen but imperatively enjoined.

So far the psychology of moral emotion, which, with tho psychology of the will, constitutes the psychological basis of ethics." 'The further problems of the validity or objective

1 While the treatment above is quite independent, its outcome in its philosophical bearings is similar to the doctrine of Herbart, its ethical implications are nearest the teaching of Sidgwick, and in psychological detail it indorses in many points the analysis of Hodgson. The similarities to the last-mentioned author's positious are so evident that the writer is only just to himself in saying that the text was written in substance for university lectures before Hodgson's Theory of Iractice (II. chaps. I-II) fell into his hands. 
grounding of ethics, the place of ethical values in a philosophy of knowledge, though belonging to ethics proper, are not within our sphere. Three distinct questions remain to ethics, points where our psychological determinations must give to philosophy a further justification of their existence: first, what is the meaning of the relative worths, which we spontaneously assign to alternative ends? Have they or have they not meaning and worth in a developing cosmos as a whole? Second, what is the authority of the moral imperative, the ought, and whence is it derived? Is it or is it not the form which the must of natural law takes on when consciousness appears upon the scene? And third, is the moral ideal essentially an illusion of my conceptual faculty, or is there an ultimate reality whose rightness will both satisfy and complete my feeble gropings after righteousness? Ethics, therefore, has three great questions, each dealing both with a feeling and with a fact, and the systems which neglect any one of the three fails of its task; i.e.,

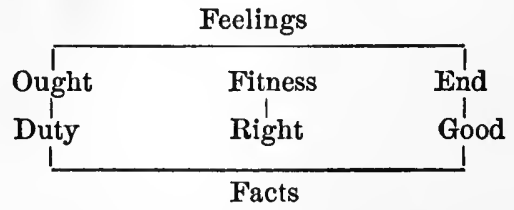

In a later connection the discussion of the ethical impulse: will engage our attention. It is sufficient now to say that, like all emotions, the ethical have an outgoing value which rests in impulse. Calling it, for the present, "love of the good," we see in it the ground of obedience or disobedience to the authority of the ethical imperative. That such a spring of action is real-whether derived or not-the difference between logical, æsthetic, and ethical feeling is sufficient to prove. ${ }^{1}$ We are constrained to do the right in a sense different from that in which we act on the consistently true or the formally beautiful.

Ethical Feeling and Happiness. The examination of Hedonism above raised the question of the true relation of happiness to the ideal element in conceptual feeling. The statement of the question from the point of

1 Compare on this point the discussion between Martineau and Sidgwick in Mind, xI and xII. 
view of conceptual feeling in general is intentional; for we are thus able to view it apart from the more particular reference to ethical discussion. In the light of later treatment of æsthetic feeling, it is clear that happiness is, in the first place, simply the tone of these emotions, as it is of others. The object which arouses logical feeling - systematic truth-is quite different from the pleasure $I$ feel in it: the pleasure is part of the feeling, not of the object of the feeling. So the object which excites my feeling of beauty is different from my enjoyment of it. The case is exactly the same for ethical feeling, unless it can be shown that the object is itself always a state of pleasure or pain-a happiness theory of the ethical ideal. It is no argument, however, to say that happiness is always present in ethical feeling; so it is in logical and æsthetic feeling: nor that most happiness is secured in the long-run by right conduct; that only means that the satisfaction arising from one impulse is more important in the long-run than that arising from others, or that happiness is the organic immanent end of mental development. These might be facts on any theory.'

As a matter of consciousness, pictured pleasure, as a motive, seems to enter the lists on an equal footing with other motives, as candidates for moral approval. It may get moral approval, for no other interest may rank above it in my present choice. But the pleasure thus morally pursued is quite different from the pleasure which arises from consciousness of right; for if $I$ do right for the sake of my own approbation, I have a clear

1 This point is recognized acutely by Sidgwick, who distinguishes happiness as the "outcome," viewed objectively, from happiness as the individual end ; yet his phrase " psychological hedonism" for the former doctrine is, I think, unfortunate, for it tends to keep up the confusion between subjective and objective ends. Loc. cit., pp. 42-44. 
feeling that my ideal has vanished and that I have missed the happiness I craved. ${ }^{1}$

Put in terms of end, happiness may be (1) a subjective natural end, and as such become morally right; (2) the organic and cosmic end, and as such justify morality in the long run; (3) the subjective moral end, in which case it is morally wrong; (4) simple tone, an ingredient of ethical feeling, not an end at all. Yet it is a peculiar fact that we often, in imagining an act, wait for the feeling of moral pleasure as an indication of its rightness before we embrace it cordially. Instead of the moral being pursued for the sake of the happiness, here is the turned-table of the happy pursued for the sake of the moral!

It is a common observation that happiness pursued is, when reached, not worth the pursuit. This is easily explained when we remember that happiness is not itself the outcome of a partieular function, but merely the tone of consciousness throughout. To pursue happiness is to do violence to the function from which it normally results. A strict analogy is found in the physical functions. Nature's design is that the heart should beat regular and strong. She secures this by my enjoyment of physical exercise. Suppose I set about pursuing the same end, to improve the heart-action: I can do so more directly by taking drugs. But the result is soon disastrous. So, undoubtedly, morality is the happiest course; it brings into harmony a number of impulses each having its tone, and adds the satisfaction of a new impulse, the moral. Further, moral feeling attaches only to moderate well-adjusted activity, which is a condition of pleasure. But as soon as I set about the pursuit of happiness, I destroy all this ground of it, and lose both the morality and the happiness. "We realize the good by conforming to the right."

Emotions Akin to the Moral. Around the fundamental moral emotions cluster a number of more special and complex feelings. Moral approval and disapproval of others in different degrees becomes moral praise and blame, moral respect and contempt, moral reverence and disgust: applied to self, they are feelings of good conscience

1 On the development of the moral feelings, see Sully, Outlines, pp. 560-67, and Bain, Emotions and Will, 3d ed., p. $285 \mathrm{f}$.

${ }^{2}$ F. L. Patton, Syllabus of Ethics (printed, not published). 
and remorse, moral hope and despair. These latter take on peculiar forms when complicated with the knowledge that others know and judge our case, i.e., moral pride and shame. These two feelings are the most powerful and lasting of our moral nature, as witness the aggravated punishment of the "Brand of Cain" and the "Scarlet Letter." They bring all the motive and emotional force of the sympathetic nature to reinforce the intrinsic sanctions of duty. Other forms of the ethical emotion whose factors suggest themselves readily are repentance, moral penance, moral restitution; and moral cowardice and hesitation, on one hand, contrasted with moral courage and resolution on the other. The great class of religious feelings are also most closely connected with ethical emotion and rest upon it.

Adequate treatment would iuclude at this point an analysis of the religions conscionsness. The limits of our plan do not permit an attempt at it; but it may be well to suggest that such an analysis is a desideratum in psyehology, espeeially as elucidating the social aspect of religious emotion. References to writers on the general subject are given at the end of the chapter.

\section{§ 8. Esthetic Feeling.'}

In beauty, the elements of what we call the ideal seem at the outset to be most fully set forth. The simplest empirical observation of beautiful things suffices to illustrate the necessity of both unity and variety in form in any object to which we attach this predicate. There is no beauty when unity is absolute, and it is only when arrangement is possible to a degree which allows a distinction between variety which is yet unity, which has a plan, and variety which is multiplicity, which has no plan-that any such feeling arises at all. It is equally evident, also, that

1 On the relation of the resthetic to the ethical feelings, see the references given by Sully, Outlines, p. 557 . 
meaning, significance, contributes to æsthetic effect. The beauty of a landscape is cold and formal until the smoke of a peasant's hut, or the spire of a country church, is added to give it a touch of human interest. The village green has more meaning than snow-clad Alps. And, further, we feel the essential sharableness, universality, validity, of all beauty. I expect a face to appeal to you as it appeals to me.

While all beauty, thus, has the ideal character, and is for that reason conceptual, yet it is well to distinguish two kinds of rsthetic emotion; that which attaches to more sensuous experience, and is almost exclusively formal, and that which attaches to more representative experiences, as having meaning. Following Wundt, the former may be called lower, and the latter higher, æsthetic feeling.

I. Lower Asthetic Feeling. ${ }^{1}$ It is difficult to determine when the sense of the beautiful begins in childlife. The expression of such a sense is for a long time simply the ordinary expression of pleasure,-smile, active muscular movements, etc.; and the presumption is that simple pleasure is all there is to express. Yet, by inquiring into the effects upon the child of objects otherwise indifferent, expressions due to form alone may be isolated. ${ }^{3}$

The objective character of æsthetic impressions leads us to look upon sight and hearing, the most presentative senses, as the exclusive organs of sensuous beauty. The objective form of sounds is time, and those of sight are time and space. The formal element,

' For interesting treatment of the rise and progress of æsthetic feeling for form, see Lotze, Microcosmus, bk. v. chap. II.

2 Spencer bases the æsthetic upon the play-instinct so early exhibited by children (Psych., II. part vIII). Sully's objections to such a derivation may be read with profit (Outlines, p. 233, note). 
therefore, in all rsthetic feeling, is unity and variety in time and space relations.

Further, in both time and space a distinction may be made, with Hodgson, ${ }^{1}$ between static and dynamic relations. Sounds which occur simultaneously, and space-relations which are perceived to be stationary, are called static; sounds following one another, and space relations which change through physical movement, are dynamic. The ordinary words for these two qualities are repose and movement.

As regards time-relations, music is the purest and most adequate illustration. In the chord, the static quality is illustrated. The variety of auxiliary tones is held in a unity dominated by the fundamental. The single tone in ordinary instruments is, further, a static effect, since in it there is also a variety of secondary or over tones which give to it its peculjar timbre. In general, musical harmony is the static form of the æsthetics of time. The dynamic element in the æsthetic feeling of time-relations is presented by rhythm, complex transitions, beat, measure, movement. It presents the formation and resolution of harmonies in a series of effects, which, while themselves static alone, yet are united in the flow of the composition as a whole or of portions of it. This dynamic aspect of the case is known in music as melody."

The æsthetic effect of music is almost entirely a matter of time form. The question of meaning in music, of the possibility of conveying emotional affections through variations in harmony and melody, is referred to below. It is clear, however, that the main and usual beauty of music is due to the distinct differentiation of sound-elements, as simultaneous or successive, which the auditory apparatus makes possible;

Theory of Practice, I. p. 186.

On the physical basis of both these qualities, harmony and melody, see the remarkable work of Helmboltz, Sensations of Tone (Eng. trans., Ellis, 2d ed.). 
together with the possibilities of union of these sensational elements secured by the activity of the auditory centre. In other words, music is mainly a physical sensational effect. In poetry, on the contrary, the form, while important, is subordinate to the meaning, as appears below.

In regard to relations of space, ${ }^{1}$ the distinction between static and dynamic, between rest and movement, is equally plain. Architectural beanty illnstrates the former; beauty of wheels in motion, birds in flight, the intricate evolutions of the dance and the drill, illustrate the latter. Considering the static quality, the question arises: What relations of space are rsthetically most pleasing? In plane figures, richness of division, together with evident simplicity of plan, is the æsthetic desideratum. A square inscribed in a circle is more pleasing than either the square or the circle; but two overlapping equilateral triangles in a circle present still greater attractiveness. Investigations have been made into the most tasteful laws of longitudinal and vertical division. For the best effect, longitudinal division should be either perfect symmetry (bisection about a. vertical axis) or some proportion well away from symmetry. Zeising's principle, called the "golden cut," is that, in horizontal division, the longer part (b) should be a mean proportional between the shorter $(c)$ and the whole (a), viz., the proportion $a: b:: b: c$ should hold. For vertical lines, it is held that the point of division should be two thirds to three fourths up from the bottom, or the same distance down from the top : as the arms on the erect human body, or the lowest broad-spreading boughs of the arbor-vitæ. The quality in division which excites æsthetic feeling we may call balance.

${ }^{1}$ On beauty of space-relations see R. D. Hay, First Principles of Symmetrical Beauty.

${ }^{2}$ Cf. Fechner, Vorschule der Alsthetic, r. p. 192. 
As regards plan, the question is largely one of outline. If the divisious are pleasing, in what kind of an outline shall the lines of a design terminate? The attempt has been made, and probably with some success, to connect the pleasure of outlines with the relative ease or difficulty of the eye-movements required to compass the figure in question.' The normal movement of the eye, except in its vertical and horizontal axes, is a curve of gentle and somewhat irregular curvature. ${ }^{2}$ Hence the general principle that curved lines present a more pleasing outline to the eye than extended straight lines. And variations of the same principle are, that curved outlines are more sgreeable when the law of curvature changes slightly at frequent intervals; that transitions should be by curves rather than by short turns or angles; and that sudden irregularities are allowable only when they can be brought under a regular law of recurrence, i.e., reduced to the general plan of the design as a whole." The erect human form has been considered from antiquity the supreme illustration of beauty of form, as regards both balance and outline.

Put more generally, the scheme of æsthetic form for the eye conforms approximately to the field of vision. The ideal of form is indicated by the most facile and pleasurable adaptation of the eye at once to detail, and, by easy transition, to the plan as a whole. Yet even as regards form, considerations of meaning work modifications, as is seen in the lack of symmetry of vertical division: this pleasurable effect of asymmetry is probably due to the instability, under the action of gravity, which is suggested by vertical bisection. An æsthetic vertical division either conforms to gravity by avoiding top-heaviness, or defies gravity by asserting superior strength of construction, support, material, etc.

The conception of æsthetic feeling as belonging in all cases to objects, that is, to presentative experience, leads us

1 Wundt, Theorie d. Sinneswahrnehmung, p. $140 \mathrm{f}$.

? Principle of Listing : see Helmholtz, Physiologische Optik, p. $457 \mathrm{f}$.

${ }^{3}$ Cf. Sully, Mrind, 1880. 
to exclude from this category, properly speaking, the pleasure that comes from certain intensities and qualities of sensations apart from their space and time form. Children in their first year show preference for certain colors, generally red and blue, ${ }^{1}$ before pleasure in forms becomes at all apparent. It is perhaps only a matter of terms to exclude such gratification from the rsthetic category; yet in adult life we are usually able to distinguish the simple pleasure of color from beauty of color, tracing the latter either to some association or to relations of figure, contrast, etc., which are aspects of time and space harmony. 'The fact, however, that color is given to us originally in space-form, makes the distinction between the two kinds of gratification liable to all the difficulties we have already found in distinguishing between objects as intensive sensational data and objects as extended and external things.

The graphic arts and sculpture, called, as opposed to music and architecture, the imitative arts, embody ideals of space-form. They are imitative only in the sense that they represent objects taken from nature; but imitation is altogether subordinate, as is seen in the fact that only such objects in nature are suited to the purposes of art which are already recognized as embodying some ideal. A painter paints a face either for its beautiful form or its beautiful meaning, or both : if it has neither, it is not beautiful as a picture of a face, and hence is not æsthetic, not art. Even a portrait must idealize somewhat, to be beautiful and satisfying.

Perspective in the graphic arts is the reduction of space-relations of depth to the form of the original field of vision in two dimensions. If it is true, it conforms to the requirements of all spacial beauty: it has a visual centre to which its lines of direction converge, and if there be two or more of these centres, they must be in turn subordinate to yet another. 248.

${ }^{1}$ See the writer's experiments reported in Science, vol. xvI, 1890, p. 
II. Higher Æsthetic Foeling. We now come to consider beauty apart from its framework of sense-perception. If space and time relations were all that resthetic ideals included, beauty would be robbed of most of its power to influence and gladden us. It is the meaning, the suggestiveness, of art that rouses in us feelings for ideals. This meaning is by many writers simply made convertible with the associations or memories which the beautiful object calls up : and in many cases this is a sufficient account. For example, a building becomes beautiful when we know that it is a hospital for sick children. The knotted hands of a workman suggest a lifetime of privation, toil, and devotion, and rouse in us emotions of respect and admiration. Yet even in cases where simple association is most conspicuous, the suggestions themselves involve ideals and seem to bring them more vividly before us. The suggested emotion does not terminate on the building, but on the ideal of charity which it represents; not on the physical hands, but on the ideal of life which they suggest. Association is, therefore, not the whole of what we intend by the word meaning. It is only as associations themselves have meaning that they enter into the meaning of present beauty.'

In an earlier place, meaning was connected with the intension of concepts. Intension includes all the data that we have about objects. But we have more data about objects than their simple presentative associates; we have also the feelings, of whatever kind, which they excite, and the motive reactions to which they impel. All these elements must enter into the framework of resthetic emotion in its higher forms, i.e., associative connections, emotional revivals, volitional and ethical reverberations.

'Cf. Dumont, Théorie Scientifique de la Bensibitité, pp. 39-42. 
And all this framework must be conceived as representative of unity in variety, harmony, universality, in a particular sphere. That is, higher æsthetic feeling arises only by the tendency of the abstracting and generalizing function to transcend its immediate presented material. The complete resthetic coefficient, like the ethical end, is an ideal and cannot, for that very reason, be given adequate formulation. ${ }^{1}$

Beauty of Truth. The more intellectual side of the æsthetic feeling is seen in our admiration for logical consistency and system. This feeling is quite distinct from intellectual emotions proper. In the latter we have relations from the producer's, in this case from the spectator's, point of view. A neat demonstration is beautiful from an objective disinterested standpoint, though I may not have the personal pleasure of thinking it out for myself. Meaning in this case is intellectual meaning, while at the same time ideal.

Beauty of Character. By this is meant harmony and proportion as prophetic of an ideal of emotion and will. Due balance of pleasures is as necessary for character as due balance of members is for truth. And the ethical feelings as already sketched give controlling color to this harmony. The most beautiful thing in the world is a man who acts harmoniously and consistently and truthfully, and finds his most harmonious and consistent pleasure in such acts. The arts, therefore, which depict phases of human emotion and conduct are the most moving in their effect, since they present an ideal meaning, which as far transcends that of sensuous form as human character transcends natural mechanism.

${ }^{1}$ A conclusion reached, though for different reasons, by Gurney, Power of Sound, chap. Ix. 
The Æsthetic Judgment Universal. Like ethical, so resthetic feeling takes form in general laws: canons of taste and art. An adequate formulation of such laws or canons would bring asthetic enjoyment down from its ideal vantage-ground. Consequently, a complete science or philosophy of art is unattainable. As far, however, as the principles of æsthetic form are reached by empirical observation, they become universal in the sense already explained.

This universality in art may be viewed under three general aspects. In the first place, it is disinterested, as all realities grow more and more to be. I idealize my gratification and make it others' gratification as well, before I call its object beautiful. Second, it is thus made matter of assertion, belief, judgment. Beautiful and homely things are realities in the most intense way. We have seen that realities are the most satisfying experiences: and what could be more satisfying than those objects which in a measure anticipate and gratify our ideals of truth and life? And third, the belief-coefficient must be consistently maintained in all the details of an art.construction. This is the extent of consistency in æsthetics. An anachronism in history or poetry violates consistency, because the coefficient of reality is fact or truth. But anachronism in drama does no violence to the æsthetic sense, because simulation, pretense, acting, is the sort of reality I go to drama to find. The truthcoefficient is not appealed to, but the imaginative coefficient. Hence the "dramatic unities" are unnecessary limitations. The principle of consistency may be expressed by saying that the kind of reality which an art construction sets out to exhibit it must consistently maintain. ${ }^{2}$

'On æsthetic judgments compare Nahlowsky's suggestive treatment, Das Gefühlsleben, pp. 163-73. 
Emotions allied to the Esthetic. The violation of certain elements in the requirements of beauty, while the other elements are present, gives rise to distinct emotions. In the comic we have violations of the law of consistency. The comic is the rsthetically abortive. A joke turns on a misplaced grammatical or logical rela. tion which, if properly placed, would have been æsthetic. A comic situation is an incongruity, where the conceptual process demands congruity and anticipates it. Hence the elements of surprise, disproportion, and disharmony, in all humor and wit. ${ }^{1}$ The comic is a matter largely of meaning. The grotesque, on the other hand, is the comic of form. The picturesque illustrates a similar departure from normal beauty, but not sufficiently so to lead to positive inconsistency. It applies especially to form, and is found in the bold, sharp, irregular, unexpected in outline. In the sublime, the meaning attaches to particular feelings, those aroused by the large, massive, forceful, and destructive; it seems also to include a coloring of fear and awe.

\section{§9. General Table of Feelings.}

The more evident distinctions of quality in emotions, as we have now found them, in connection with various conscious functions, may be thrown into a table for general review : it is meant to be exhaustive, but not minute.

${ }^{1}$ Not only is the comical the unexpected, as Dumont maintains, Causes $d u$ Rire, but it is that which we have no right to expect, which we have every right not to expect; and negatively, the sense of fun often arises from the simple absence or failure of what we do expect. Another requisite is that the subject be trivial and involve no important consequences. We often say an erent would be ludicrous if it were not so serious.

'For other classifications the reader may consult the references given. at the end of Chap. III. 

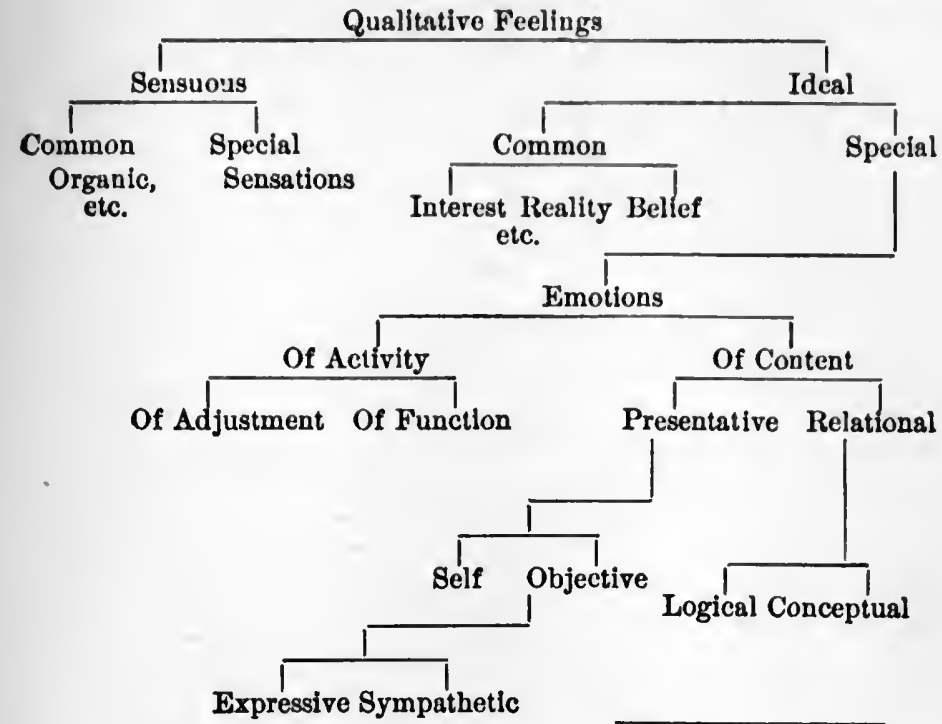

Systematic Ethical Esthetic (Religious)

On logical, ethical, asthetic, and religious emotion, consult: (ethical) Perez, l'Education dès le Berceau, pt. 1. chap. 7 ; (formal) Waitz, Lehrbuch, pp. 295-320; Wundt, Phys. Psych., 3d ed., II. pp. $209 \mathrm{ff}$. and $424 \mathrm{ff}$.; Volkmann, Lehrbuch, $\$ \$ 133$, 134; (ethical) Martineau, Types of Eth. Theory, II. bk. I; Nahlowsky, Das Gefuhlsleben, pp. 157 ff.; Dumont, Théorie de la Sensibilité, pt. II. chap. v, II, Iv; Sully, Outlines, chap. xII, and Pessimism, chap. xI; Spencer, Psychology, II. pt. IX. chaps. V-IX; Bain, Emotions and Will, pp. 215293; Drbal, Lehrbuch, \$§ 110-113; James, Sentiment of Rationality, Mind, IV., 317; (æsthetic) refr. given by Dewey, Psychology, p. 325; Lotze, Outlines of Fsthetics; Guyau, Problèmes de l'Esthétique contemporaine; Bradley, Ethical Studies, III.

On the comic: Dumont, Causes du Rire and Thérie de la Sensibilité, pp. 202 f.; Lévêque, Revrue des deux Mlondes, Sept. 1863; Hecker, Physiologie u. Psychologie des Lachens.

Further Problems for Study :

Relation of resthetic and moral feelings ;

Development of rsthetic and moral feeling ;

Theories of the arts ;

Ethical theories :

The religious feelings ;

Canons of art criticism; cultivation of taste ;

Social obligations and duties ;

Psychology of crime, of punishment, of charity. 


\section{CHAPTER X.}

\section{QUANTITY AND DURATION OF EMOTION.}

\section{§1. QUANTITY OR INTENSITY.}

Mental Excitement. The most general predicate which we can make of the states of feeling arising about mental operations is expressed by the term excitement. The word means stimulation, and as physical stimuli bring about a more or less diffused physical reaction or bodily excitement, so presentations, ideas, stimulate higher states of feeling in forms all of which exhibit the diffused property called excitement. If we picture a logical machine, with no feeling whatever, turning out syllogisms, we picture at the same time the absence of that excitement which makes the mind in its logical character different from such a machine. "Coolness" is the popular word- "calmness" is more fitting-to denote. the absence of emotional excitement. For the present, we will consider such excitement on its conscious side, and call it mental, leaving the question of its relation to nervous diffusion until its inner aspects have been. pointed out.

Relativity of Feeling. The general nature of feeling, as dependent upon physical and mental processes, accounts for its extreme variability in different and in the same circumstances. If feeling arises everywhere in consciousness, the present state of feeling must result from a great complexity of bodily and mental conditions. 
The variability of feeling may be spoken of under several so-called principles.

Principle of Contrast. In general, this principle means that any state of feeling is pure only when it is isolated as regards quality : it is a general extension of the principle of contrast found in connection with sensation-qualities.' Such isolation is quite theoretical; but any feeling-quality stands clear only as it does not share consciousness with other feeling-qualities. Sounds are louder and clearer during silence, colors are distinct and well-defined in proportion as no other colors appeal to the eye with them. Pleasures are more pleasurable after or during pains. Hopes and fears exaggerate each other. The whole affective life is a matter of contrasts of sensational and emotional qualities.

In the emotional life auy regular observation of contrast is impossible, because of the extraordinary complexity and variety of emotions. Just as any sense-quality may be modified by a new or concurrent nerve-stimulation, so an emotional quality may be modified by any variation in the intellectual or ideal complex which constitutes its stimulus. The principle of Hering cited above, i.e., that the apparent union of sense-qualities is really a union of stimuli, not of sense-qualities-aids us also in understanding complex forms of emotion. We cannot consider the half-hope, half-fear state of mind with which we watch a friend who is desperately ill, as being literally the presence of two or more qualities, hope and fear, influencing each other. That would mean that we had first a clear picture of the friend's. death, then of his health, and so on in alternation; also that the two nervous processes were separated from each other. On the contrary, the state is one, anxiety, an emotion arising from the intellectual complex of doubt 
as a whole. Here there is a mental compound or summation, as in the case of color-contrast there is a nerrous summation.

That there is a nervous summation in the emotional complex is also true; but from the analogy of the foregoing treatment of feeling, the intellectual summation is the immediate ground of the complex emotion. Of course the conscious state itself has a neural basis, and the physical expression of emotion shows that the excitement has also. But waiving for the present the question of cause and effect as applied to emotion and its expression, we still have a right to consider the intellectual complex as the conscious stimulus to the emotion, and to find in it, by analogy from Hering's principle, the ground of emotional contrast.

Principle of Attention. The general law of attention in its relation to mental intensities has already been cited: attention heightens the intensity of any mental state. This is especially true of states of ideal feeling. It is so easy for an interesting idea to tarry in the focus of consciousness ; it is so hard to withdraw a conception which promises advantage, from our mind's consideration; that the emotion which such an ideal presence arouses becomes lasting and influential. We calm an angry man, or an angry headache, by arousing interest in a new subject; we work up curiosity or hope by keeping the expected revelation vividly forward; we cure disappointed love and allay crushing grief by travel or by business details which absorb the attentive energies.

It is a curious fact, however, that after an emotion has reached a certain degree of intensity, the absence of the object only inflames it. It is analogous to the fact noted in connection with happiness, i.e., happiness is more acute in the pursuit than in the possession. It is probably due to the dissociating influence of the imagination, which keeps only the particular details to which the emotion attaches, in consciousness; while if the object be actually present, extenuating and modifying features are forced upor the attention. 
There is a limit, however, beyond which we must cease to speak of the influence of attention upon emotion and reverse the terms of the proposition. Intense emotion bids defiance to the laws of mental control, carries the attention perforce, and paralyzes the intellectual life. Yet the liability to this result depends upon the quality of the emotion. It is seen at once that emotions which depend upon complex constructive intellectual processes must be strongest when these processes are at their best. An inventor or an artist feels most strongly when he thinks most deeply, and, further, his emotion is a spur to his invention. So strong interest, which arises when apperceptive concentration and adjustment are smooth, reacts to further the play of thought. In general, therefore, we may distinguish the presentative, expressive, and sympathetic emotions as those which interfere most with thought. They present a fixed ideal content, an image, a fact, an event, hold the attention to it, and thus obstruct the normal flow of consciousness. This different relation of various emotional states to intellection, confirms both the principle of dependence of emotion upon ideation, and the details of the foregoing classification of the emotions.

Principle of Accommodation. A corollary of the principle of contrast is found in a class of facts which show the abatement of emotional excitement under long-continued stimulation. Sensuous feeling, both pleasurable and painful, as we have already seen, is modified if the organic stimulus be continued. The reason is that the organ becomes accommodated to the stimulus. After fatigue begins, however, the rule is reversed. The same is true of ideal feeling, both as a matter of immediate adjustment of the attention (mental accommodation), and in view of the conditions of mental habituation. The emotions of adjustment die out under conditions of 
easy attention. As regards habituation, the law is that already pointed out in connection with the emotion of interest, i.e., interest as excitement, present emotion, is attendant upon exploration, but dies out as association passes over into fixed habit. So emotional excitement generally subsides when the stimulus is often repeated. Yet we tind that repetition strengthens the deep-seated tendency to emotion, i.e., passion, ${ }^{1}$ just as it strengthens deep-seated interests.

Principle of Fatigue. The law of accommodation leads directly to that of fatigue. Accommodation has its limits. As, in speaking of sense-qualities, we found that a long-used function tends by fatigue to disappear in the rise of a new function, so the attention, and ideation generally, has its corresponding emotional relativity. Intellectual fatigue attaches not only to the attention, as an activity, but also to its content. We tire of a subject and our emotion loses its freshness and strength: and the following emotional state has, through contrast, more than its ordinary force. That this contrast-effect also has its physiological basis in some form of dynamic cerebral summation appears from Mosso's experiments, ${ }^{2}$ which show that mental is distinct from muscular fatigue, although the two have a direct influence on each other. We may suppose the seat of an emotion to be a given ideational centre: prolonged stimulation of this seat exhausts it and predisposes the centre to react in a manner corresponding to a new ideational product and emotion. This form of emotional contrast is exaggerated in certain mental diseases which are due to damage to the coördinating centres of ideation. The emotions become as facile and fickle as the play of ideas.

1 See below, p. 257.

See above, Chap. IV. \$ 2. 
Emotional Expression. Like all other states of feeling, the emotions belong in the reactive consciousness. As forms of excitement they represent conditions of intense stimulation, and find their physical basis in processes of pronounced nervous change. As excitement simply, apart from qualitative differences, emotion indicates a diffusive outgoing wave of nervous action consequent upon heightened processes in the centres of ideation. Viewed qualitatively, the particular emotions are correlated to nerrous discharges in particular directions and portions of the nervous apparatus, issuing in muscular contractions to a large degree differentiated and peculiar. Such muscular indications of emotion are most clearly marked in the face, though the more intense extend to the limbs, and finally take the form of massive and convulsive movements of the trunk. So familiar are we with these forms of emotional expression, and so expert have we become in reading them, both from experience and by heredity, that our responses to them are instinctive. Only the practised observer is able to analyze the common facial indications which we all readily construe in terms of answering emotion.

A good deal of progress has been made by psychologists in assigning to the different emotions their peculiar correlatives in the muscular system. In general, each main emotion expresses itself, not by the contraction of a single muscle, but of a coördinated group of muscles. The smile or weeping of an infant is, at the start, a matter of very extended muscular inuervation, and in adult life the entire countenance seems to take on the semblance of thought or laughter, and to support the brow or mouth in its assumption of the leading rôle. The general facts of the case, as respects the leading presentative emotions, are readily observed by noting others, or by simulating emotion before a mir- 
ror: it is unnecessary to go further into details which are endless and wearisome.

Sir Charles Bell ${ }^{1}$ is one of the pioneers in the exact study of emotional expression, and he is well supported by the more exhaustive work of Darwin. ${ }^{2}$ Some of Darwin's descriptions have become classic, and have not been surpassed by later and more elaborate researches. Further references are to be found at the end of the chapter. The hypnotic state, especially the condition called by the Paris school catalepsy, affords a striking method of studying expression. Either an emotion or its expression may be suggested to the hypnotic subject. ${ }^{3}$

The fundamental emotional expressions are impulsive. The child inherits the necessary vital reactions for its life and growth, and, besides these, certain muscular contractions indicative of pleasure and pain, joy and sorrow, i.e., smiling, weeping, crowing, sobbing, etc. Very early, more distinct emotions grow up with corresponding ready-formed reactions-fear, wonder, anger, love, jealousy, etc. It is probable, from what we know of mental growth, that the rise of these early emotions waits upon the development of their appropriate nervous basis: which means that it waits upon the development of certain ideational centres.

Such general emotional expressions are either elevating and exciting, or depressing and inhibiting. Besides these regular native reactions, however, which are common to individuals, there are multiform peculiarities in the motor side of feeling, every man having, externally, his emotional stamp. We all have habitual movements -twitching, nervous contractions of face, hands, limbs, often anything but comely, postures of the body as a whole-which were probably at first no more than acci-

${ }^{1}$ Anatomy of Expression, Bohn's Library.

Expression of the Emotions.

${ }^{3}$ Compare Binet and Féré, Animal Magnetism, p. 277. 
dental paths of discharge for the diffused wave of nervous excitement. These paths become fixed by recurrent discharges. "Temperament" is judged externally largely by such indications.

The attempt is often made to determine what emotional expressions are fundamental. Jessen' names joy, sorrow, anger, and fear ; Bain, ${ }^{2}$ love, fear, anger. The question is of little importance, except as enabling us to pass judgment upon the order of development of the emotions; for if the forms of emotional expression may be reduced to two or three, it does not follow that the emotions are capable of a corresponding reduction. It is interesting, however, from the evolution point of view." If belief is the fundamental general emotion of conscious reality, and if attraction and repulsion are the fundamental forms of impulse, then perhaps the special modes of impulsive belief may be considered original expressions, i.e., joy and sorrow (belief with past reference), love and anger (belief with present value), fear and hope (belief with future reference). All these expressions are inherited, as observation of infants shows; though it would not do, perhaps, to say that no others are.

Theories of Emotional Expression. The traditional theory of such reactions is that they are literally expressive: that they follow as effects upon emotions considered as intellectual eauses. If we were able to assume that the mind is a substantive self-sufficient entity, giving and taking influence to and from the brain, such a conception would be tenable. Empirically, however, we are not able to justify such a conception. Our experience is of emotion expressed, not of emotion and expression. If we endeavor to quiet the expression, to inhibit the nervous reaction, just in the degree in which

1 Versuch über Psychologie, p. 295.

Emotions and Will, p. 72.

3arwin's laws of development of emotional expression have become classic. He held that the principal expressions resulted from the fixing by inheritance of muscular movements serviceable to the life of the animal organism. For this and his subordinate principles, be must be consulted; Darwin, loc. cit. chap. I. 
we succeed, the emotion vanishes also. Even in the case of the intellectual emotions, the muscular accompaniments of attention are always present. Clearly, therefore, psychology has no more right to an effect theory of emotional expression than to an effect theory of the cerebral changes which accompany conscious phenomena in general.

On the other hand, the cause theory of emotional expression reverses the order of events and makes the so-called muscular expression cause of the corresponding emotion.' This is equally positive, as a metaphysical conception; since it gives primacy to the physiological basis beyond the positive evidence at hand. Even though it be true that any emotion may be calmed by the inhibition of its nervous expression, it is equally true that the nervous diffusion may be calmed by the play of association or the passage of attention. And as for chronological priority, all our analogies from psychophysics show that such priority is unlikely on either side. All attempts to prove priority are vain, considering the fact that muscular expression is peripheral, while the nervous basis of emotion is central. Suppose it proved that the muscular reactions either precede or follow the conscious emotion, what will follow regarding the central process? The analogies spoken of afford us ground for a sounder theory of the physical basis of emotion.

Physical Basis of Emotion. Conceiving the problem of expression under its widest reach, the view required. both by the physiology of the nervous system and by the facts of consciousness, comes plainly out. Let us call the aspect of nervous processes which belongs peculiarly to emotional excitement, the nervous coefficient of

1 This theory is most interestingly presented by James, loc. cit., Ir. chap. xxv. See also C. Lange, Über Gemüthsberoegungen. 
emotion, substituting this phrase for the question-begging word expression. The question then is: In what kind of a nervous process does this coefficient consist? What nervous process varies-rises and falls, grows or contracts in extent-with corresponding variations in conscious feeling?

Now in the general conception of the nerrous system stated above, we found that personal consciousness was present only when the system attained high integration. We have also found that sensibility is only another name for consciousness : intense consciousness is intense sensibility or excitement. Excitement, therefore, is the kind of consciousness which arises when nervous integration is intense, i.e., very complex and very unstable. This is the nervous coefficient of emotion. Emotional expression is, then, the outgoing side of the nervous coefficient. Complexity at the centres means diffusion in discharge; instability at the centres means facility of discharge-just the two characteristics of emotional expression.

And how inapplicable on this view are questions of priority! High nervous integration expressing itself outwardly, conscious excitement felt inwardly-these are the data. One cannot be removed without destroying the other; how then can one precede the other? The two things are there together; what has psychology to do with the question of cause and effect as applicable to them? To push the question farther is to go into speculation on the origin of consciousness itself, i.e., why is it that there is a coefficient at all, and which coefficient, the nerrous or the conscious one, is the metaphysical prius?

It is not necessary, either, to inquire what kind of molecular activity the nervous coeffieient is. The complexity side of it is best conceived in terms of the dynamic connections between brain-centres which we 
found necessary for the physical basis of the intellectual function. In saying that emotions of various kinds spring from combinations of ideas, that we have ideal emotions as well as sensuous feelings, is only to say that the cerebral coefficient of emotion includes that of presentation. But the emotion is not thereby removed from its own cerebral process, and made to follow it.

Further, since the common ideal feelings-interest, reality, belief-give most general form to conscious emotion, so the physical basis of excitement is most general when these common feelings occupy consciousness. Reality-feeling has its nervous basis in the process which underlies consciousness generally ; interest indicates both greater complexity and greater mobility in cerebral state; and belief implies a new mode (or intensity) of brain-function, taking form in the various belief-coefficients recognized above.

The sensational coefficient carries, besides intensity in the proper sensor seats, some kind of nervous modification corresponding to sensations of resistance and effort, possibly to be sought in the phenomena of nervous inhibition. The logical coefficient suggests activity in the coördinating centres which physiologists are endeavoring to locate; and the ethical and æsthetic coefficients, though akin to the logical, are among the many subjective facts for which nerve-physiology gives us as yet none but speculative and unsafe analogies.

Conscious Diffusion of Emotion. The element of diffusion already pointed out in the nervous basis of emotion is a marked characteristic, also, of mental excitement. Strong emotions spread themselves out over the whole content of consciousness, and our thoughtcurrent becomes grave, gay, elevated, depressed accordingly. Not only so, but we objectify our feelings to an extent. The external world takes on the color of our mood. This is probably due to our lack of control over strong emotion: we are unable either to banish it or to 
pin it down to its peculiar object. It is also especially true of the more deep-seated organic conditions which give tone to consciousness as a whole. Dyspepsia is the most notorious enemy to good spirits.

Brain-seat of Emotion. Assuming the central apparatus to be made up of sensor and motor elements and dynamic connections among them, we find three alternatives open to us regarding the locus of the nervous coefficient of emotion. Either a peculiar manner (or intensity) of activity originating in the sensor (ideation) centres is felt immediately as emotion, passes off through the motor centres, and is reported back to the sensor centres as feelings of muscular expression (Fig. 14, $A$ ); or the emotion-coefficient may originate in either the

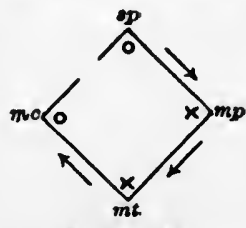

A. Theory 1.

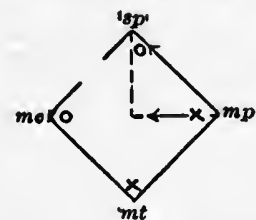

$B$. Theory II.

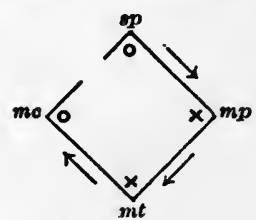

C. Theory III.

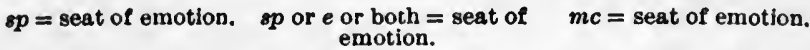

Fro. 14.-Brain-seat of emotion: "motor square." The sign $x$ signifles a brainprocess, the sign o consciousness, at the point at which it occurs.

motor or sensor seat, and be carried back as emotion, either to the ordinary sensor seats or to a seat of its own ( $e$, in Fig. 14, $B$ ), without the intervention of the muscular movements of expression; or such a process may originate either in the sensor or motor centres, but not be felt until it issues in muscular expression, the consciousness of which is the emotion (Fig. 14,C). Of these alternatives the first and second may be combined in a view which best represents the present state of the evidence, i.e., the emotion-coefficient is felt at once in consequence of the stimulation of the centres of ideation, just as the sensation-coefficient arises, giving 
qualitative sensuous feeling, upon stimulation of the same centres. This stimulation is communicated to the motor centres, which react back upon the sensor, and also issue in muscular movement. The motor element felt in emotion, as far as it is not due to association from earlier muscular expression, is due to the setting back of nervous disturbance from the motor to the sensor centres.

In support of the first theory, as modified from the second, and in opposition to the third or "kinæsthetic" theory, especially as defended by James and Lange, it may be sairl: 1. The analogies from sensuous feeling, sense-qualities, are all in favor of the central origin of emotion. No one holds that sensations are felt only as far as they have motor expression. 'The kinæsthetic theory accordingly forfeits unity in its account of sensibility. 2. It is generally admitted that the nervous process of ideation (reproduction) is a reinstatement of the sensational process; we would expect, therefore, that special feeling-conscionsness would accompany the former as it does the latter. 3 . The cases where motor effects seem to follow directly upon presentations, the emotion coming later, are worthless as evidence $;{ }^{1}$ for, as already said, to prove priority of the peripheral reaction proves nothing of the central processes. It would only show that the motor discharge is more facile than the emotional discharge, and this might be due either to the distraction of attention and violent interference with the mental process which the emotion accompanies, or to the time required for the nervous process to "set back" from the motor centres. The argument is a post hoc ergo propter hoc. 4. Argument from "those pathological cases in which the emotion is objectless" " is equally inconclusive ; for they are consistently accounted for on the theory that the emotion and the expression are common effects of disease in the centres of ideation. 5. Further, the kinæsthetic theory is inadequate to account for suggested emotion, "suffering by hallucination," etc., in anæsthetic patients." The actual expressionthe kinæsthetic factor-is absent, yet the suggested idea

1 James, loc. cit., II. pp. 457, 458.

${ }^{2}$ Ibid., II. pp. 459-62.

'James, Ibid., II. p. 456 ; cf. his chap. viII, in vol. I, and Janet, Automatisme psychologique. 
brings a real emotion. It will not do to say with James that there may still be sensations in a split-off consciousness which contribute to the major emotional consciousness, for two reasons: first, it is difficult to see why processes which cannot get into consciousness when they are stimulated from the periphery should still be able to get in when they are set moving over the very same courses by ideally initiated muscular movements ; and second, to say that such processes do get in to the motor consciousness and prevent ataxia is to assume that the cause of ataxia is the absence of kinæsthetic stimulations, which is not proved. Further, the abrupt rise and fall of these suggested emotions points us to the ideal process with its accompanying attention as their real seat. 6 . Such suggestions account for the fact urged by James that expression increases emotion; the expression suggests more emotion by ideal suggestion; it is also true when the expression is some one's else expression.'

Emotion and Passion." Jessen asks the question whether emotion is real when it is not felt, i.e., do we love our friends when we are not thinking of them. The answer to this question introduces us to the great class of facts covered by the word passion. By passion is meant the growth of emotion in depth at the expense of expression. What we have already learned of physical and mental habit would lead us to expect a consolidation of emotions in a few great habitual forms of reaction : for this is what we find both in the nervous organism and in the intellectual life. Nerrous

1 Janet's suggestion, loc. cit., p. 215, that the kinæsthetic stimuius may come in from sight and hearing, really supports the centrai theory, since such stimulations, as he himself holds (p. 274), instead of giving direct emotion, rather suggest it by association. Prof. James seems to me to give up his whole case in saying (loc. cit., II. 459, note) : "Under these conditions (trance, ecetasy, ordinary dreaming), one may have the liveliest subjective feelings... of the emotional sort, as a result of pure nerve-central activity . . . with complete peripheral repose."

'The word passion corresponds to Kant's Leidenschaft. The Germans use Affekt to cover emotion as excitement.

${ }^{8}$ Versuch über Psychologic, p. 296. 
reactions become organized in subconscious motor intuitions; mental reactions become organized in perceptions, subconscious beliefs, and interests : so emotions take on mentally subconscious forms. They become so habitual as to be unremarked except when some new occasion calls them out in the shape of emotional excitement. A man's love for his fiancée is a matter of constant consciousness and expression: his love for his wife-it takes a burning house or a drowning-accident to bring fully into his consciousness. Emotional excitement, however, remains the method of expression of passion, and in popular speech the term passion is given to such violent expressions themselves. The real passion, however, is deep-seated prevailing emotional motive; it enters profoundly into our notion of character.

Among the most marked passions, some are clearly inherited, others may be traced in their development from occasional recurring experiences of emotion. The most distinct classes of passions may be designated affections and sentiments. Affections arise from the more interested and personal classes of emotions: examples are sympathy, love, contempt, benevolence, stoicism, pessimism. Sentiments spring rather from the more objective, disinterested emotions : examples are reverence, respect, religious or irreligious attitudes, love of beauty, morality, etc.

Theories of Emotion. Theories of expression involve and are involved in theories of emotion. Three general views are held as to the nature of emotional excitement: intellectual theories ${ }^{1}$ hold that all feeling is ideal feeling, taking its rise from the relation of ideas to one another as opposing or reinforcing forces. This theory fails confessedly ${ }^{2}$ to account for sensuous

${ }^{1}$ Volkmann, Horwicz, and the Herbartians generally.

${ }^{2}$ Nahlowsky, Gefühlsleben, pp. 61-63. Cf. Waitz, Lehrbuch, pp. 306, 
feeling. Physiological theories ' make all feeling sensuous feeling, in compounds of varying degrees of complexity. Emotion is a higher form of organic pleasure and pain-a biological function. This theory fails to account for higher emotion, or, indeed, for feelingqualities generally. It involves a doctrine of unity of composition throughout the entire affective life. Original theories " are opposed to these in holding, in some form, that feeling-qualities are original subjective facts. The entire foregoing exposition of feeling is an argument for the "original" view.

This classification, again, is a broad psychological one, and takes no account of the particular views of individual writers, many of which are extremely interesting. ${ }^{3}$ As regards expression, an intellectual theory of emotion carries an effect view of expression ; a physiological view of emotion carries a cause theory of expression; an original theory may stop short of either view of expression, or it may reconcile the two in a conception of concomitance or "coefficient" akin to that in the text.

Reproduction of Emotion. From what has been said of the conditions of the rise of emotion, the laws of its reproduction are evident. If emotion is present only when an ideal object is present, and if an ideal object is present only when the brain-conditions of earlier sensation are reinstated, then the laws of association of ideas with their basis in dynamic cerebral processes are also the laws of the revival of emotional excitement.

In consciousness the dependence of revived emotion upon revived ideas has the same evidence as that of

307, who reproaches Herbart for distiuguishing between feeling and the perception of it.

' Spencer, Bain, Schneider, Ribot, Lewes; see Ladd's exposition, Elements of Phys. Psych., chap. Ix.

- Spinoza, Hodgson. Wundt. James.

For example, Paulhan holds that emotion arises from the repression or inhibition of impulse; Les phénomènes affectifs. 
first-hand emotion upon presentations, i.e., the evidence of invariable concomitance. Among these ideas, however, we find remembered muscular and organic sensations. I may reproduce grief either by recalling a grievous event or by throwing my countenance into the form of grief-expression. If I fail to get one of these, I fail to reproduce the emotion.

Further, we would expect the suggested emotion to vary as one or another coefficient of reality attaches to the revived experience. When an event is remembered and recognized as a real event in my past life, the emotion it arouses has a new quality from the fact of its present real setting. I may remember my past object of wrath with present gratitude or affection, my past hopes with present regret, my past fears with present complacency. The revival of the original emotional coloring of an idea depends upon the permanent worth of that idea as I first experienced it. Or I may voluntarily banish my present flow of thought, reinstate all the conditions of the first experience, and thus bring back the original emotion. In case of the memory of sensational experiences, the reality-feeling is much stronger and the same emotion comes back with more or less force. This is because the object is in these cases bound more closely with my own feeling, and with difficulty put in a new emotional setting.

As far as the same emotion is revived, it is not simply a picture of a former state, but a real state of feeling. When I remember a pain, I am in pain : but not neces; sarily in the same pain. For example, I remember vividly : toothache: I have a real pain at present, but it is not a toothache. By the fact of memory, it has lost its sensational coefficient, but it has the memory-coefficient, and is real. It may by its intensity become a real toothache, i.e., get its sensational coefficient again, thus becoming an illusion. The picturing of the facial elements of ex- 
pression is the most immediate representative means of awaking similar feelings-a widening of the fact already noted of the emotion of sympathy.

This affords an explanation of what is known as the contagion of emotion in crowds, and on a broader scale, in common sentiments in communities and states. In a crowd, fear will spread with amazing rapidity, probably by the semi-unconscious interpretation of muscular and vocal expression. So the styles of taste, morality, and custom are inhaled, so to speak, from the emotional atmosphere in which we live.

It is a remarkable fact that revived emotion has a cumulative quality which tends to produce the intensity and reality of illusion. The law of summation seems to apply peculiarly to emotions which are kept in consciousness and thought of. Not only is my present state of revived emotion intense from the activity of attention, but it seems to have further intensity simply from its duration, and this further force in its turn tends to keep the attention enchained. So emotion and idea react and reinforce each other.

Transfer of Emotion by Association. It is a matter of clear experience, also, that emotional excitement gets transferred by association to ideas by which it is not originally aroused. The color black has become doleful and sad from mourning associations, the sight of the postman in the morning brings joyful emotion; in fact, interests of the deeper kind, as has been already remarked, arise from the expenditure of emotion or action upon things at first uninteresting. The whole range of symbolism and suggestiveness in art rests upon this fact of accrued feeling, when the ideas from which it has accrued have become vague or subconscious.

It is much debated whether the object with which emotion is thus associated ever becomes intrinsically of emotional worth : some hold that to the last the emotion only arises in as far as the associated idea which at first aroused the emotion is more or less consciously suggested. The particular case of the miser who grows to love his gold is in point. Does he lovo only the pleasure, power, etc., which the gold stands for? While such a complex state of emotion as ararice may remain 
in doubt, ${ }^{1}$ yet there are simpler analogies which point to the real transferrence of feeling. Physically we have such facts. as fainting at the sight of blood when no association with blood has been acquired; aversion to certain animals, as. snakes, lizards, etc. The only explanation is to be found in the supposition that race-experience is registered in the nervous system, and the necessary life-preserving reactions attach themselves to the simple associates of real dangers. In the sphere of emotion also we find analogous aversions, antipathies, fears, etc., which the individual is quite unable to explain from any association in his lifetime: and we must hold that the emotion often terminates upon innocent and intrinsically neutral presentations.

Conflict of Emotions. All mental conflicts are conflicts: of feeling. So-called conflicting ideas are those which are felt to be in conflict, i. $\theta$., those which introduce conflict into the life of feeling. So the much-talked-of conflict of feeling and reason is purely a conflict of feelings. Reason here means the moving aspect of thought, the strength of truth in setting the subject into action. I might apprehend a truth clearly and yet find no conflict between it and my life which denies it. It is only as it moves. me, as I have an emotion for it, that it makes a conflict for supremacy. But emotional conflicts are real and tragic, especially when they play around questions of duty. And it is the degree of persistence and strength of the underlying ideas that gives and takes the victory. Emotional conflicts, therefore, indicate the hold that various kinds of truths have upon the agent. One man surrenders to the sensational coefficient, the sensuous; another gets an easy victory for the distant and ideal; while a third lives a life of irresolution or decision according to the accidental appeals of one truth or another.

1 On this case, which illustrates the ethical bearings of the subject, see Flint, in Mind, vol. I. 


\section{Duration of Eyotion.}

The experimental method of determining the duration of simple psychic acts is not applicable, in any exact way, to emotional excitement: for the reason that the psycho-physical process is not here a matter of simple nervous reaction or of the abrupt come-and-go of presentations. Emotious are diffused excitement, both internally and exterually. All that can be said, therefore, of the duration of emotion, pertains to the general conditions of its rise. It is, of course, only a truism to say that emotions last only as long as their causes last, but the twofold basis, physical and intellectual, of emotion gives the truism some special bearings. Cases are recorded of the absence of the intellectual object and the continuance of the emotion, its expression being obtrusive and vehement. It is less frequent, but real, also, that emotional expression may be apparently lacking, as in intense rsthetic, ethical, and spiritual feeling.

Emotional Cessation and Relief. It follows also, from the foregoing, that relief from emotion may be artificially courted. Indulgence in strong outbursts of feeling tends to allay their causes; it exhausts the nervous processes involved and induces other emotions. Knocking a man down satisfies my feeling of revenge more from the new emotion of justice or houor vindicated, than from nervous expenditure; but both satisfactions are real. Relief by nervous expenditure follows, especially, in cases of emotion which excite to action. It is always a relief to have done something in an emotional emergency, whether it be successful and wise or not.

Again, there is a great class of emotions which sharing tends to relieve. Novelists make much of the smouldering motif in the growth of feeling. The immediate effect of sharing a personal emotion is to temper it by the sense 
of sympathy and social community. Psychologically, several elements enter into this sense of relief : a feeling arises that the friend confided in justifies and defends the emotion; also, a feeling that help and support are secured. And there is further relief by the cessation of the feeling of isolation and loneliness, which is the reverse of social feeling.

All this offers new proof of the general utility and social value of sympathy. Not only is it pleasurable to suffer with another, by the greater value of the social over the egoistic impulse; but one of the keenest and most elevated enjoyments of life is the pleasure of being sympathized with. This is akin to the additional pang imported into remorse and repentance by the knowledge that others know and condemn. The same is also evident in the pleasure of vanity, which transcends pride through the consciousness that the praise of others is added to one's own.

Relief from sharing is, however, temporary unless assisted by other agencies. And the return of feeling is more intense from the sense of social support. Apart from its immediate effects, which are largely nervous, sharing deepens emotion by fixing the ideal causes in the attention, expanding the reasons for feeling fully in consciousness, and giving additional associations to keep it constantly in mind. Mourning garments, cards, etc., undoubtedly keep grief alive. We often have emotions because we feel that it is expected of us. ${ }^{1}$ Yet often one of the old associations that has long seemed the dried channel of a forgotten joy or grief, empties upon us an overwhelming flood of sweet or bitter memories. Such experiences we call revulsions of feeling, and they sometimes give a new turn to the permanent current of the affective life.

1 When nine years of age, the writer lost a brother, and his memory of mourning is largely of his consciousness of the importance of the occasion and his desire to do himself and his family credit by his deportment. 
Emotional Rhythm. The alternating relief and recurrence of emotion gives it a certain periodicity or rhythm; not as definite as the rhythm remarked in connection with the attention, but probably resting in part upon it. Ideas sufficiently inteuse and important to arouse strong emotion stay in consciousness inclependently of continuous attention. They hold the attentiou in spite of its attempts to liberate itself. Further, emotion is a massive thing which diffuses itself over the entire content of consciousness, and dominates the mental life in the form of a mental mood. Minor changes have little influence upon it. The rhythm of emotion is, therefore, a deep-seated thing, depending, as far as its physical conditions are concerned, largely on the rise and fall of the rhythmical organic functions, or of the vital energies as a whole. Emotions play themselves out, but while we sleep they gather their secret forces.

On mental excitement as emotion, consult: Spencer, Psych., §211; Hodgson, Theory of Practice, I. bk. I. chap. II ; Bain, Emotions and Will, pp. 3-293, especially chap. III ; Hume, Treatise, bk. II ; Schneider, Thierische Wille, Cap. iv ; James, Princ. of Psych., II. chap. xxv; Spinoza, Ethics, in loc.; Ladd, Elements of Phys. Psych., chap. Ix; Dugald Stewart, Active Powers, vol. I; Höffding, Outlines, vi. E, F; Ward, Encyclop. Britann. ; Volkmann, Lehrbuch, $\$ \$ 127$ -132 ; Sully, Outlines, pp. 480 ff. ; Garnier, Traite des Facultés de l'Ame, bk. Iv. chap. v; Paulhan, Les Phénomenes affectifs et les Lois de leur Apparition.

On emotional expression: Bain, Senses and Intellect, 277-88; James, loc. cit., II. chap. XXV; Ladd, loc. cit., chap. Ix; Wundt, Phys. Psych., 3d ed., Ir. 504 ff.; Warner, Physical Expression; Dumont, Théorie de la Sensibilite, pt. II. chap. vI; Schneider, Mensch. Wille, chap. xx; Spencer, Psychology, §261, and I1. pt. Ix. chap. IV; Sully, Sens. and Intuition, pp. 23 ff.; Ferrier, loc. cit., pp. 67 f.: Mantagazza, Physionomie et l'Expression; Mosso, La Peur ; Carpenter, Ment. Phys., chap. vir. 


\section{CHAPTER XI.}

IDEAL PLEASURE AND PAIN.

The earlier consideration of sensuous tone leads up to a clear view of higher or ideal pleasure and pain. In general, the phrase ideal tone is used to designate the pleasurable or painful quality of emotional excitement. We are now able to gather up the mental conditions of pleasure and pain as we before sought out the physical conditions. A preliminary retrospect of the two great classes of emotions, i.e., those of activity and those of content, ${ }^{1}$ or formal and material, both of which have pronounced tone-quality, leads us to see that new factors now come into play; factors not involved in sensuous feeling. The phenomena of sensuous tone are adequately explained from the consideration of function only, with its adjustments. Such an explanation of ideal tone, it is plain, would apply only to one class of the emotions, those of activity, which we have found themselves to fall in two classes corresponding to function proper and adjustment. The tone of the whole important class of emotions of content must have some further explanation. Considering first the tone of the emotions of activity, we may call its conditions the primary conditions of ideal pleasure and pain; the conditions of the tone-quality of the emotions which attach to objects, to a content, will be secondary conditions.

The need of this distinction and its great importance will appear more clearly as we proceed. Heretofore the explana-

${ }^{1}$ Above, Chap. VIII. § 1. 
tion of pleasure- and pain-quality has stopped with a theory of physical tone from which an analogy was drawn as to the nature of ideal tone. But-to cite an example without anticipating the discussion-what analogy from the physical conditions of pleasure and pain is applicable to the pleasure of beauty or the pain of envy? 'The new condition in these latter cases is the presence of the object in consciousness-a condition of which bodily pleasure and pain, as such, know nothing.

\section{§1. Primary Conditions of Ideal Tone.}

1. Some degree of ideal change. As physical pain arises from physical function, so higher pain comes with apperception considered as ideal function. And in general, the degree of ideal function, measured in terms of the emotional excitement to which it gives rise, indicates also the degree of pleasure or pain. Ideal change, the rearrangernent of elements in the apperceptive content of consciousness, is accordingly the general condition of particular ideal tone.

We may, therefore, at once-subject to support from empirical observation-make use of the conception of sensuous tone already arrived at, substituting for the physical, the apperceptive function, and for the adjustment of end-organs, that of attention; and expect to find an adequate conception of ideal pleasure and pain. Accordingly we reach a second condition.

2. The degree and duration of attention: determining ideal tone as pleasure or as pain. Excessive concentration of the attention is painful; yet the pain involved is directly merged in the pain involved in the adjustment of the bodily organ. Prolonged attention becomes painful by the law of fatigue already found operative upon emotional states. On the other hand, moderate concentration and duration of attention are pleasurable. The "emotions of activity" mentioned above, give emotional body to these states of pleasure and pain. 
3. The degree of adjustment or misadjustment of the attention. The conditions given above as involving emotions of distraction, etc., are painful; those giving feelings of ease, flow, variety, etc., are pleasurable. It is probable that the most pleasurable adjustment is that of finest and most exact discrimination. Ward formulates this and the preceding condition as follows: there is pleasure "in proportion as the maximum of attention is effectively exercised."

Strict analogy, accordingly, from the philosophy of the sensuous functions, leads us to define ideal pleasure as the conscious effect of that which makes for the continuance of the apperceptive life or its advancement; and ideal pain, the conscious effect of that which makes for the decline of the: apperceptive life or its limitation.

\section{§2. Secondary Conditions of Ideal Tone.}

The determinations already reached have evident: application to emotions of activity, to those states of feeling which arise around acts of the attention regardless of the nature of the object to which the attention is directed. The other emotional states, however, are pronounced in their contribution to the tone of consciousness. The great expressive emotions (fear, love, anger), the sympathetic, the ethical and æsthetic, are all at times controlling agents of pleasure or pain. The question at once arises: Is it possible to bring them under. the formulas already enunciated? This question awaits.

1 Loc. cit. In this formula the pleasures of activity are well formulated, as against the plurality of energies of the faculty-psychologists: (Hamilton); but it is open to the same criticism that the Herbartian theory is, i.e., that it takes no account of the pleasure and pain occasioned by objects presented. My painful fear of a mad dog is hardly due to my failure to give him attention. On the contrary, the more effectively he culls out the exercise of my attention, the more pained I grow. 
an answer from the consideration of the conditions under which objects come to be pleasure- or pain-giving.

1. Oljects of perception excite pleasure or pain only as they have some present or future relation to our physical wellor ill-being. Perception, as has been seen, is a summing up, a synthesizing of sensations; an object gives us certain sensations, and these enable us to anticipate others. The sight of falling rain prophesies to me the unpleasantness of being wet; the sight of a lion, the pain of being eaten. The tone of perception, therefore, as far as it refers to the object, is intrinsically a prophecy of the tone of the sensations it includes and suggests.

Put more generally, the tone of the qualitative expressive emotions has reference to the preservation of the animal life. Fear means danger to life, love means satisfaction and advancement of life; and if it be true that this law of life-development is a sufficient account of sensuous pleasure and pain, then we find it possible to account for the tone-quality of the expressive emotions by the same principle. Sensations, in which knowledge synthesis is implicit, have toue-quality-an index of the present life-process: perceptions, in which the knowledge synthesis is becoming explicit, and which has the value of future sensations as well, have tone-quality -an index of the future life-process.

To illustrate : a child first sees a fire (yellow-light sensation), grasps it (touch-sensation), feels pain (sensuous tone, due to damage to the life-process). Again he sees the fire (perception, carrying in it touch- and painmemories) and has fear, which is of painful tone. The point advanced is that this latter tone, of fear, also has. reference to the life-process. It is nature's way of utilizing simpler pain-experiences, just as perception is her way of utilizing sensational experiences.

This is not to say, however, that ideal pleasure and pain, as given in expressive emotion, is reducible to sensuous tone. 
It is not. It arises only when the process of perception is present. I may be pained by the glare of a printed page, but at the same time pleased at the thoughts I read from it. Yet it means that in both sensation and perception a common life-process runs, to which both are subservient, and this lifeprocess comes to consciousness in the pleasure and pain which is common to both. This consideration applies to the other forms of ideal tone considered immediately below.

In the case of the sympathetic emotions, a difficulty seems to arise from the fact that one's own life-advantage is not secured in the pleasure of sympathy. Yet when we remember that at first sympathy has no personal object, but is simply an emotion of suggested pleasure or pain, we see that the principle is the same as for fear, anger, etc. When the child has the pain of sympathy, it is because he feels again his own former pain, and that pain is at first sensuous pain. As for the tone of sympathy with intellectual and emotional distress, not sensuous, that comes under the same head, provided it can be shown that such sympathy reflects the well- or ill-being of the physical as well as of the mental life.

\section{Representations of objects excite pleasure and pain only} as the objects themselves excite them. This covers the whole field of emotions due to reproduction-memory, passive imagination, illusions, etc. The emotions which such representations excite have qualitative coloring (expectation, dread, etc.), but their tone is again due, as the tone of perception is, to the anticipation of advantage or damage from the pictured object.

It should be noticed that the emotions whose tone has been so far considered, all involve the sensational coefficient of belief ; they all terminate on external objects presented or represented. Some such conclusion as this is accordingly reasonable, i.e., the pleasure or pain of belief in the external world is the indication in consciousness of the worth of particular aspects of that world for the individual organism; a proposition which may be further extended by this consideration : that through perception and memory the organism is capable of adjustments to conditions more remote in time and space. The animal that only feels, lives and dies in a closeencasing milieu: a creature that perceives and remember's, is forewarned by the past and anticipates the distant and future. 
3. The tone of the conceptual and relational emotions has reference both (1) to physical and (2) to intellectual wellor ill-being.

(1) The reference of conception and thought to physical pleasure and pain is clear in some cases. My conception of the work of dentists, for example, has a painful tone which is as clearly a warning of physical damage as the perception of my particular dentist is. So, also, the science of dentistry, the logical framework of the art, considered merely as a branch of instruction, caunot be rid of its physical suggestiveness. The medical student grows faint when he hears his first lecture on blood-letting. Consequently, a positive part of the tone of higher æsthetic, ethical, and logical emotion illustrates the law of physical well-being.

In the case of resthetic emotion, the element contributed by association is largely of this sensational character. Apart from the beauty of the purely sensuous in music, its associations are largely sensuous. A face often becomes handsome from association at the table, the theatre, on the promenade, and the pleasure we take in it is a reverberation of these associated pleasures of sense. The ethical we have also found to involve tone-quality, and in the absence of higher motives, the physical is often the right. It is our duty to preserve our health, and it is impossible to separate the pleasure arising from such an act of duty from the memory-tone of health itself.

Again, it shonld be pointed out that higher tone is a new thing, not the same as physical tone, although it subserves in part the same law of physical development. The esthetic and ethical emotions are distinct qualitatively, and arise only from explicit intellectual and voluntary operations; and their tone can only arise when they themselves arise.

It is also true here that the organism, by thus entering into the region of thought with its claim to consideration, gets new and marvellous facilities of adjustment to its living con- 
ditions. Who can say how soon our organic life would be snuffed out if we did not make reasonable provision for its protection and prolongation? We must live in order that we may think, love, labor, it is true: but, first of all, we must think in order that we may live.

(2) So far, it is clear, we may carry a naturalistic view of pleasure and pain, conceding that, whatever purpose they may serve beside, all normal pleasures point to healthy, and all normal pains to unhealthy, physical functions. Does this exhaust the range of ideal tone? Further consideration convinces us that it does not. There are emotions whose tone seems to violate the law of physical well-being. It is a common distinction-that between higher and lower pleasure: and so obtrusive that a great school of moralists ${ }^{1}$ have attempted to justify the popular consciousness in the matter, by making the distinction a fundamental one in the ethics of the moral end. This attempt we have found to be unsuccessful, since it is not tone which has quality, but it is rather mental quality which has tone. That is, it is in the conditions of pleasure, as higher and lower, physical and ideal, not in pleasure itself, that a distinction is to be made.

We would expect, if consciousness is a synthetic thing, and if its synthesis becomes explicit in what we call apperception or thought, that such a new thing in nature would have its own principle of development. And we would expect, further, that its development would be a matter of conscious adaptation to free conditions of thinking and willing. The most natural view of ideal pleasure and pain, therefore, is to consider it an index of healthy or unhealthy mental function. As physical pleasures, at first ministering blindly to the welfare of the organism, grow to attach to objects in

1 The disciples of J. S. Mill. 
relation to the organism; so ideal pleasures, while attaching still to attention as a function, yet come to attach to its objects as well. On this view, the tone of many emotions, as the conceptual and relational, reflects the state of the mental functions primarily.

This surface-view is supported by abundant empirical evidence. The pleasures of intellectual pursuit lead their devotees to neglect the body and even to continue this course in the face of acute physical pain. Esthetic delight is so independent of selfish motives that admiration is often called out by what is destructive and terrifying. Ethical emotion, with the happiness it always brings, may triumph over physical impulse, when they come into conflict. In all these spheres, further, the possible antagonism of pleasures is a matter of such direct conscious experience that philosophers, from Plato down, have supposed a great gulf fixed between the animal soul and the rational, the gratification of the latter alone being true happiness. As to the actual decision of consciousness on the question of a distinction between the conditions which bring happiness and those which bring pleasure, there can be no doubt.

Accordingly, we find it necessary either to maintain a dual reference of ideal pleasure and pain-to physical well-being on one hand and to intellectual wellbeing on the other-or to find a postulate upon which these two aspects can be shown to be included in one truth. Without appealing to metaphysics, we may simply conclude that ideal tone refers to personal wellbeing as a whole. And it is equally true that sensuous tone refers to both physical and mental well-being. Consequently, no violent separation between the two interests is possible. If mind develops with body, and body with mind, then their interests, as represented in consciousness, would be generally common interests. In the relative adjustments of their claims, the ideal, by 
common consent, takes precedence ; but why this is, and what it means, let philosophers decide.

Philosophy finds it easy to suggest two hypotheses, either of which empirical psychology, as far as this single difficulty is concerned, should be free to accept. The materialistic evolutionist identifies physical and mental well-being; the mental life is a form of consciousness resulting from a highly integrated brain-process; and since the end of brain-development is complex integration, the pleasure of higher ideation and volition is primarily an index of physical development. The spiritualistic monist, on the other hand, says the highest outcome of evolution is mind; once born, it is the evident goal of nature. Physical pleasure is only a prophecy, a stepping-stone, to the higher happiness which is nature's provision for the perfection of spirit. One is the philosophy of mechanism, of which thought is only the most complex expression: the other is the philosophy of reason, for which the mechanical is but a preparation.

The same conclusion may be reached from the point of view of impulse, of which pleasure is the gratification. It will appear later that the intellectual ideal impulses stand out as something different from physical impulses; yet that the two cannot be set over against each other, as answering different purposes in the economy of the individual life. The will is amenable to both, and both go to make up personality.

\section{§ 3. Final Conclusion on Pleasure and Pain.}

Summing up all that has been said of pleasure and pain, both. sensuous and ideal, we may conclude that pleasure and pain are the affective coloring, respectively, which consciousness takes on in conditions of present or prospective well- or ill-being: ${ }^{1}$ a definition which can be understood only in the light of earlier ${ }^{2}$ explanations of the terms which it employs.

And we have found three classes of conditions upon which pleasure and pain depend: first, physical conditions, giving sensuous tone; second, ideal conditions,

1 Cf. Lotze's statement, Microcosmus, I. p. 240.

Chap. V, and the foregoing sections. 
giving rise to the tone of states of attention; third, a union of physical and ideal conditions, giving rise to the tone of the emotions of content.

\section{4. Universal Presence of Tone.}

It is now possible to justify the earlier assertion that all mental states have tone, that present consciousness is never quite indifferent as regards pleasure and pain.

It may be well to remember that consciousness itself is primarily sensibility. Whatever happens in consciousness is therefore felt, whether it be mental excitement, knowledge, or volition. But this sensibility may not itself become a matter of consciousness, i.e., I may not know that I am feeling cognition or volition; they may not intrude themselves upon me as states of feeling. In other words, feeling may be distinguished from consciousness of the fact of feeling. This latter we have called excitement, i.e., feeling so intense that it " ruffles up" the surface of consciousness. For example, an accountant works up his books for the thousandth time; his entries and additions are performed semiautomatically; he is not aware of any excitement, any consciousness that he is all the time feeling the processes he performs. But he comes upon a deficit in his accounts; something does not balance. At once the meaning of the deficit becomes a problem of felt importance and interest, and every subsequent step of his calculations is accompanied by a consciousness of feeling, i.e., by excitement.'

Now it is simple sensibility, intensity of conscionsness not sufficiently high to become a matter of consciousness, that oftenest seems to have no coloring of pleasure or pain. Positive excitement, it is safe to say, always has such a coloring; and wo may say conversely

'See the exposition of H. M. Stauley, Mind, xrv. p. 538. 
that positive recognized pleasure and pain always indicate excitement.

Bain's cases of "neutral excitement" have been sufficiently examined by Sully ${ }^{2}$ and others. Bain instances surprise, restlessness, wide-awakeness, and other analogous states, as indifferent. An adequate analysis, however, shows a distinct hedonic value in them all. The tone of surprise runs so quickly into the pleasure or pain of the event anticipated that it is difficult to isolate it. But as pure excitement, surprise seems to fulfil the law by which moderate stimulation is pleasurable. The tone of excitement is generalized popularly in the phrase "love of excitement." The further fact urged by Bain and noted by Sidgwick, ${ }^{3}$ i.e., that the intensity of pleasure and pain is not commensurate with the intensity of excitement, finds a ready explanation in the fact that excitement is cumulative. It summates by addition; while pleasure and pain tend to neutralize each other in some instances. An alternation of hope and fear, for example, throws consciousness into intense excitement, while at least four elements of tone are present, confusing and annulling one another, i.e., pain of suspense, pleasure of excitement, pain of fear, and pleasure of hope. The excitement is stimulated by all the elements together; the tone reflects now one, now another. But when the others subside, the pleasure of excitement comes out alone. That pleasure and pain may be acquiredas pleasure in horse-racing-only goes to show that the worth of the object of the emotions may change, not that excitement has no constant tone.

In regard to general sensibility-consciousness of each mental event for itself, apart from the added consciousness of excitement-the case is somewhat different. In the case of many sensations, complete indifference is claimed. A simple sound seems to give neither pleasure nor pain. The sight of a button on my coat is indifferent. This position is further strengthened by the probable fact, already mentioned, that physical pleasure and pain involve distinct nerve elements or processes; and

1 Mind, xII. 376, and XIv. 97.

3 Mind, XIII. 248.

${ }^{3}$ Methods of Ethics, pp. 45 and 48. Of. also pp. 178-94. 
that sensations of pressure, temperature, etc., may be entirely separated from feelings of pleasure and pain.

This might be valid as far as pure sensation is concerned; but it must be remembered that in our ordinary lives pure sensation is a fiction. Even in the simplest bodily feelings, if they occupy the attentive consciousness, an element of synthesis is involved, which has its own law of tone. The hedonic condition is one of mental function as well as one of bodily well- or ill-being, and while the nerves of physical tone may be isolated from the sensational process proper, we know of no way to isolate and destroy that element of the hedonic consciousness which colors the apperceptive process. Consequently we are thrown back upon the inquiry as to whether intellectual operations are ever without some hedonic value.

Empirically we are here in the presence of the phenomena called subconscious, least conscious, and unconscious. Many of our perceptions and thoughts seem to pass before us with no interest and no tone; we can recall the thoughts, but we are as indifferent to such recall as we were to their original presentation. If there were pleasure or pain, it has lapsed into the subconscious. Undoubtedly Waitz is right in saying ${ }^{1}$ that if any mental states are indifferent to us it is those which are of no worth to us. But it may be asked in reply, with Lotze: Are any states quite worthless? What I see with indifference now was once a pleasant novelty or a painful shock. Every new adjustment of the attention carries evident pleasure of activity, and each subsequent adjustment differs from the first only in the degree of its affective coloring. As Lotze sums the matter up, "we can conceive no state not either in harmony with the conditions of psychic development or somehow contrary to them." " 
Accordingly, when we speak of indifference, we mean practical indifference: lack of excitement, not lack of tone. To many, felt indifference is itself very pleasurable, and is much cultivated. As soon as we begin to theorize we find that "active neutrality," to use Dumont's phrase, is all that can be justified. But we do find here, as elsewhere, that consciousuess is a matter of relative intensities, and that pleasure and pain, like presentations in general, may be so completely out of attention as to seem out of consciousness. 'The evolutionists reject pure indifference for the reason that it wonld serve no purpose in the development of the organism.' 'The further argument (Wundt) that pleasure shades down into pain, giving a point in the curve which is neither,-i.e., indifferent,-is ingeniously met by Höffding, who cites the fact established by experiments to determine the threshold value of sensation, that the point of neutrality between pleasure and pain varies according as it is approached from above (by diminishing pleasure) or from below (by diminishing pain).

Complexity of Tone-states. It is now clear that the hedonic coloring of consciousness, at any time, is not a simple thing. Pleasure or pain are reported from the body and from the mind, from many organs of the body at once, and from many mental "moments" at once. Hope and fear may be struggling within, the will may be painfully paralyzed, attention distracted, and with it all, a beating sun may annoy, an aching tooth distress, and all go to make up a complex condition of tone. So mental and physical conditions may combine to produce pleasure; and all possible combinations may, and do, arise in kaleidoscopic order.

The elements, however, of this complex effect may be generally distinguished in consciousness. They do not coalesce except in their general tendency to produce emotional excitement, which has its own tone. If the two hands be held under two streams of water, very hot and pleasantly cool, respectively, the two hedonic effects may be clearly distinguished from each other. So, as

1 Schneider, Thier. Wille, p. 95.

2 Outlines, p. 287. 
already pointed out, the pain of suspense arises from the excitement of alteruating hope and dread, and persists apart from the pleasure and pain of those emotions themselves as they struggle in consciousness.

The maximum happiness of the individual wonld be the harmony of all these conditions, in reporting each its maximum of pleasure, and the maximum pain would be the reverse. But this maximum would vary with individuals, according to the relation of these several springs of pleasure and pain to one another, and according to the degree of culture attaching to each. In heaven, the child would lose nothing from perfect happiness because the saint was immeasurably more happy. Probably our happiest condition is one in which we alternate between activity in which there is no thought of self, and sudden inward glimpses of ourselves by which we discover how happy we are.

Relativity of Ideal Pleasure and Pain. What has already been said of the relativity of general ideal feeling holds also of ideal pleasure and pain. Each is made more vivid by contrast with the other. Ideal hedonic states are further, more lasting and important than physical ; because, probably, the intellectual conditions are more revivable than the physical. The possibility, also, of artificially acquiring pleasure is greater in the case of ideal states. ${ }^{1}$ Farther, of the two ideal states, pain is more vivid, acute, and permanent than pleasure-what we would expect by analogy from the similar relation of sensuous pleasure and pain."

On feeling as indifference, consult : Marshall, Mind, xIv. p. 511; Bain, Emotions and Will, p. 13, and Mind, xı. p. 376; Stanley, Mind, xIv. p. 538 ; Sully, Johnson, Mason, Mind, xıı ; Nahlowsky, loc. cit., p. 15 ; Lotze, Microcosmus, I. p. 243 ; Dumont. loc. cit., p. 297: Sidgwick, Methods of Ethics, pp. 178-194; Bouillier, Plaisir et Douleur, pp. 86-88 ; Hamilton, Metaphysics, pp. 525-27 ; Ladd, references, Elements of Phys. Psych., pp. 509, 510.

I Cf. Spencer, Psychology, \$128.

- Bain, Emotions and Will, p. 107. 


\section{PART IV.}

WILL.

MOTOR ASPECTS OF SENSUOUS FEFIING.

\section{CHAPTER XII.}

THE MOTOR CONSCIOUSNESS.

§1. IDEA of the Motor Consciousness.

THE general notion of the motor consciousness has already been made more or less plain. By it is meant the ensemble of elements in consciousness, contributed in any way by the motor apparatus. If there be consciousness of the condition of the motor areas in the brain, of the process of the outward flow of the nervous current, of movements taking place or having taken place-all are elements of the motor consciousness. The phrase, therefore, is most general; and it may be defined as consciousness in as far as it is concerned with muscular movement. Its further differentiation and analysis are questions left open for any hypotheses which may be able to make good their claim.

The motor consciousness is, therefore, simply consciousness as filled up with a particular class of feelings or revivals of such feelings. These feelings have already been roughly distinguished ${ }^{1}$ from other elements of the general sensibility. They are thereby, also, set apart as feelings, affective states; the fact that they are asso-

${ }^{1}$ Muscular Feelings, Chap. IV. $§ 2$, above. 
ciated with movements is simply an added fact to the statement of their intrinsic nature as feelings.

Law of Mental Dynamogenesis. Empirical observation tends overwhelmingly to confirm the inference we would expect from the law of nervous dynamogenesis, i.e., that every state of consciousness tends to realize itself in an appropriate muscular movement. The nervous application of the law leads up at once to its application to sensibility. If every ingoing process produces an outward tension, or tendency to muscular discharge, and the more intense and integrated conditions of central stimulation be most delicately adjusted to such a play of incoming and outgoing processes; then we would expect the sensible modifications which accompany these more complex conditions to tend to a form of consciousness peculiar to the motor reaction. That is, we would expect the affective consciousness to merge into the motor consciousness, just as the ingoing nervous process tends to the discharge of energy into the outgoing courses.

The analogy, therefore, may be put something like this: the nervous system in its development has taken on the two functions called stimulation and reaction. When consciousness arises, it is at least-whatever else it be-an aid through pleasure and pain to the lifeprocess, and to the further development of the system." Analogy would lead us to look, therefore, for this new factor in connection with each of the two essential nerrous fuuctions, stimulation and reaction.

So much may be fairly said apart from the consideration of the details of the motor consciousness; that is, simply from the actual fact of such a thing as the motor conscious-

1 Above, Cbap. I. $\$ 2$.

The hypothesis is quite in order here, that this further develop. ment of the nervous system is for the sake of consciousness, i.e., mind. 
ness. The interpretation of the details may show that the analogy does not hold in this form : that, after all, the motor element is only a qualitative element, distinguishable, as other qualitative elements are, within the consciousness which accompanies the sensor-nervous process. At any rate, it is certain that some of our motor feelings are such, i.e., the class called above kincesthetic.l. If this possibility be confirmed, then innervation'-feelings, as a class distinct from kinæsthetic feelings, will disappear. It is enough, however, at this point, to make it plain that the nervous analogy is on the side of feelings of innervation: and that, if the contrary is proved, it must be by evidence strong enough to overthrow this analogy. And the strongest proof, apart from fact, would be to show that an improved conception of nervous development may dispense with an element of consciousness belonging to the reactive function of the system, as such.

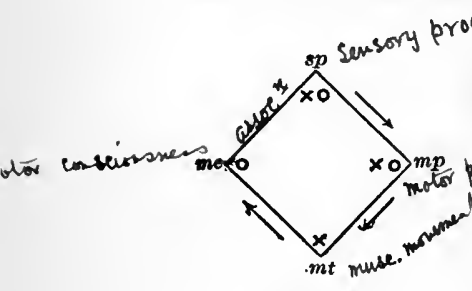

Fra. 15.

The concomitance of the nerve processes and their conscious states, if the above analogy holds in a simple way, is shown in Figure 15, which represents the normal motor consciousness by means of the "motor square."

Varieties of Motor Consciousness. If it be true that all states of consciousness tend more or less strongly to bring about appropriate muscular reactions, we should find several phases in the motor consciousness. And this is true. It is, indeed, one result of this truth, as empirically observed, that we are able to say that all states of consciousness have a dynamogenetic influence. It is our task, accordingly, at this point, to trace the motor bearing of the different kinds of consciousness which have been already distinguished, i.e., to discuss the motor value of the subconscious, of reactive and of voluntary consciousness, respectively, as far as this value is felt.

1. Fuling of a movement aftar it thes place.

1. Teeling fa movernest before it takes plece. 


\section{§ 2. Motor Value of the Subconscious.}

The facts already adduced to illustrate subconscious phenomena are largely motor facts. Motor phenomena which fall below the threshold of conscious reaction belong partly to the subconscious and partly to the unconscious; that is, partly to very weak sensibility and partly to sentience. But it is impossible to draw a line of distinction between them, and the attempt to do so would be quite artificial. We shall, therefore, mass such reactions together under the above heading, claiming, at the same time, license to include those reactions which are, as reactions, purely nervous.

In the case of a subconscious reaction, we come across the curious fact that a nervous process itself insufficient to call out sensibility may have muscular effects which are quite sensible. We respond to stimuli which we do not discern, and which we fail afterwards, perhaps, to discover by introspection. We often speak or write words which we do not mean and have not been thinking of. Associations often lack conscious links. We respond to a settling chair by balancing the body, to differences in the material we tread upon, by increased muscular tension. In short, close observation leads to the conclusion that we are constantly alert to our surroundings when we are apparently unconscious of them. The whole class of coördinating reflexes already described belong here. The most unmistakable class of cases covers suggestions made in the hypnotic state, which are carried out many days afterward in the normal state, the individual being unable to give any reason for his action. In this case, we seem to have absolute unconsciousness, a physiological reaction apart from any modification in the major consciousness, whatever we may say about the existence of a secondary conscious- 
ness. There are other states which are very vaguely or dimly conscious, such as presentations of objects, or memories of events, so habitual as to be reacted upon without attention. We walk about our own house, hang our hats up, and rub our shoes, and then, when asked, are unable to tell whether we did any such things or not. We wind our watch at night, and learn the fact later only by trying to wind it again. We suddenly discover ourselves half-dressed in the morning in garments we had decided to wear no more. We take a walk, forget our errand, and soon "rein up" in the most unexpected part of the city. In the horse-cars we brace ourselves from the jolting, move up and give another half the seat, and often pay our fare without taking our attention from the morning paper. A nervous man will arrange his necktie or stroke his mustache fifty times a day without "knowing" it, and all of us have our little motor habits, which we are conscious of but do not observe. Perhaps as clear a case of a direct adaptation of our movements to objects of which we are only passively conscious is the way we pass about in a well-filled drawing-room when wrapped in thought, avoiding all obstacles by a most circuitous and irregular route.

It is only by license that the physical type of reaction is here in point. Its explanation is found in the law of consolidation or downward growth of the nervous system. When a sensori-motor connection or nervous arc becomes so free in its reaction as not to involve a higher centre, its stimulus excites it, but does not arouse consciousness. The reaction, however, is reported to conscionsness in terms of muscular movements, whose effects are members of other more complex arcs; we know, that is, that the movement has taken place. Automatic and simple reflex nervous reactions, therefore, supply the physiological basis of subconscious sensibility-as far as there is any sensibility accompanying such reactions. In general, any reaction performed in entire independence of the attention belongs to this class. 


\section{§ 3. Motor Valee of the Reactive Consciousness.}

The reactive consciousness has already been characterized with sufficient clearness. It is marked off from passive consciousness by the presence of a reaction of the attention, i.e., by the presence of reflex attention.' The term reaction brings out clearly the fact that, in such case, the attention is in response to an unexpected and unpremeditated stimulus. As has been said above, there is just as truly a reaction in consciousness as there is in the nervous system, although the elements of the reaction are often thrown out of their true order when taken up into the discriminating process. For example, I hear a loud unexpected sound, and turn my head involuntarily in the direction from which it seems to come. The order of events appears to be this : first, the sound; then my sensation of sound; then the attentive impulse carrying with it, first, the grosser movements of the head and trunk, and, afterwards, the finer movements of the eye-muscles, etc., engaged when the attention is concentrated; then a discrimination of the sound through the attention; and. finally, a motor response to it. This is not the order, however, in which I myself apprehend or recognize the different elements in the reaction. About the first thing I know in such a case is that I have suddenly turned my head and body, and am concentrating my attention upon something which I now subsequently learn to be a sound.'

1 The relations of these so-called kinds of consciousness to attention may be illustrated as follows:

$$
\text { Consciousness. }\left\{\begin{array}{l}
\text { Passive ........... Diffused. } \\
\text { Reactive.......... Retlex. } \\
\text { Voluntary......... Voluntary. }
\end{array}\right\} \text { Attention. }
$$

- This subversion of the serial order has an interesting illustration in psychometrical experiment: the experimenter often reacts, and only learns by a later act of discrimination that he has mistaken or anticipated the signal (sound). 
Elements of the Reactive Consciousness. Here, again, we must refuse to abide by the intellectual apperception view, and go back to the feeling view of the case. Taking the reaction, then, as a felt reaction, and considering its elements in the order made typical in cases of nervous reaction, we find, first, a stimulus to the reactive consciousness, i.e., whatever affective or feeling element in consciousness calls out an involuntary act of attention; second, the feeling of expenditure in an act of attention which is drawn out without volition, whatever this feeling may be found to include; and third, the feelings of the muscular movements appropriate to the particular stimulus.

Of these three elements of the reactive consciousness, the last may be considered as comprehending only the feelings of movements already executed; that is, feelings coming in from the grosser muscles of the body, etc. These constitute a clear kinæsthetic contribution. to the motor consciousness.

The stimulus in this form of consciousness is treated in a later connection: so it remains for us to inquire into the feelings which properly belong to the act of involuntary attention itself, so-called feelings of expenditure. And they must be considered independently of feelings of voluntary effort: if we are able to reach a coherent. conclusion regarding expenditure alone, it will be of great. service to us when we come to consider effort.

\section{§ 4. Feeling of Expenditure in Attention.}

Description. A description of the sensuous feelings: which go to make up the consciousness of movement. has already been attempted. It remains to ask whetherthe phenomenon of involuntary attention confirms that description, and whether, further, any light can be thrown upon the feeling of the act of attention itself. 
Inspection of such an act of attention leads to the detection of the following elements.

1. Feeling of Readiness to Attend: Mental Potential. Such a feeling of readiness or potential has already appeared in connection with muscular movement. Muscular freshness and vigor pervade the entire organic system : so readiness to give attention or to do intellectual work is a clear and well-marked state of consciousness. And the two seem to be, in part at least, distinct from each other. After confining myself to my writingtable all the morning, my attention loses its elasticity and readiness of concentration : but my muscular system begins to feel an overabundance of energy, a pressing readiness for exercise. And when I give up my intellectual task and indulge my craving for exercise, I have a peculiar feeling of throwing off the mental weight, of getting rid of the thraldom of ideas, in the easy enjoyment of muscular activity.] However we may account for it, the difference in consciousness between feelings of intellectual and of muscular potential is well marked. Intellectual readiness probably includes both central nervous and muscular freshness.

2. Feeling of Fatigue of Attention. The state of the case is about the same between intellectual and muscular fatigue. The question whether there is nervous fatigue apart from the fatigue of particular muscles has already been adverted to. It is difficult to divide this question in two parts and suppose purely intellectual fatigue apart from nervous fatigue. The feeling of fatigue in attention may be taken, provisionally at least, to include, first, fatigue of the nerrous system, either in the sensorium as a whole, or in the particular elements which are brought into play in the activity which occasions the fatigue, and, second, an element of lowered muscular tone.'

${ }^{1}$ Cf. MLosso, Archiv für Anat. und Phys., P'hys. Abth., 1890, p. 89. 
Considered in this way, fatigue, as a feeling, is the opposite of the potential feeling: that is, it indicates unreadiness, or low potential, in the attentive activity. And the two would seem to find their explanation in the same hypothesis. The sequence of these feelings, therefore, is at first sight the same as the sequence of the muscular feelings of potential and fatigue. ${ }^{1}$

3. Feeling of Activity in Acts of Involuntary Attention. The central point of expenditure is reached in the feeling of actual activity during the attention. If readiness precedes the attention, and if fatigue follows it, what feeling do we have during it-at the very moment of it? Is there a feeling of activity, apart from the feelings of all kinds now described? Postulating such an element of consciousness at this point, and remembering the corresponding postulate made above of a feeling of nervous outgo, or activity, it remains to examine both postulates, and further, to see if there be any ground for the opinion that the two postulates represent one and the same fact.

Sensorial and Intellectual Attention. Further, involuntary attention is either sensorial, i.e., terminating on a part of the body or on an object, or intellectual, i.e., terminating on an image. The case of the sound which causes a start is typical of the former : the play of images in passive imagination, or revery, when all control is withdrawn, illustrates the latter. The question before us may be put separately for these two cases: and the word "thought" will be used to designate the play of ideas in apperception, apart from any voluntary influence we may have over them.

The problem of the feeling of attention is thus simplified, and three plain questions now confront us : First, are we conscious of nervous outgo from the brain, or is 
our consciousuess only of the effects of such outgo? Second, are we conscious of an activity of attention or thought, or only of the effects of such an activity, that is, of thinking thoughts, or only of thought thoughts? And third, are these two forms of consciousness one and the same thing?

\section{§5. Theories of Feeling of Activity in Reflex Atrention.}

The questions thus put are answered consistently among themselves in two great ways, indicating two schools of thought. Philosophical conceptions cannot be excluded here, both because the unreflecting consciousness itself supplies the conception claimed by many to be most philosophical, and because the facts are so extraordinarily obscure and hypothetical that hypothesis alone is able to cope with them. On one hand, the solutions of the three questions are gathered into what we may call the postulate of a principle of associated effects, and, on the other, into that of a spiritual principle.

I. Association or Effect Theory of Reflex Attention. This theory regards reflex attention as an associated mass of incoming muscular feelings and memories of such feelings. It accordingly holds, in answer to the third question, that the feeling of muscular expenditure is the same in kind as that of expenditure in reflex attention, the feelings involved in the two cases arising from different classes of muscles and muscular memories. Thus feelings of ordinary expenditure are from the limbs moved, with their traces left in memory; while feelings of attention are from certain muscles of the eye and eyebrow, skin of the skull, etc., with mennories of former acts of attention.

Without citing detailed evidence this position seems 
well made out: it suffices, in proof, to observe that the feeling of muscular expenditure is not present when the attention is entirely absent. If my arm is raised mechanically by a friend who comes softly behind me and grasps my hand, I do not feel muscular expenditure; the feeling is quite absent. After many such movements, I begin to feel fatigue, it is true, but it is clearly muscular fatigue: an effect reported by the afferent process. The same is seen in cases of sudden twitching of the muscles, due to isolated discharges in the brain, and in pure reflexes: they are known only after their occurrence. Consequently the third question may be thus disposed of.

In the next place, this theory replies to the second question above, i.e., Are we conscious of the activity, process, of thought, or are we conscious only of the products of thought; of thought-relating, or of thought-relations? The answer is that we are conscious only of the latter, of thoughts after they are thought. Whenever we catch ourselves thinking, either we feel that we have just thought something, or that we are just going to think something. There is no process between the absence of the second term of my thought and its presence, no gap at all. For example, a loud sound calls my attention; there is no interval of conscious thinking, no feeling of thinking, between the absence of the sound and its presence. The whole case is a succession of feelings thrown into temporary confusion by a new feeling, and the eureka-feeling that results, when I recognize the sound, is only the fortunate circumstance that the series ends in a feeling that is familiar. Even granted, moreover, that there is a synthesis in thought, yet it is known by the presence of such synthetic constructions in thought, not by any consciousness of the process of making them.

This point, again, seems to be well taken as regards the actual elements in consciousness at any given stage 
of thought. It is a mistake to say that we feel a syn. thetic activity in consciousness when volition is absent: all that we feel is the coming together and disjoining again of elements. That this is the result of an activity is an implication, a recessary presupposition, not a felt fact. The analytic method consists in taking a something found in the mind and asking how we got it: if we were conscious of the process of getting it we would not need to ask this question.

The only apparent difficulty with this position is to account for the different degrees of ease or difficulty, slowness or rapidity, of thought in different circumstances. But the advocates of the effect theory have a ready answer in the analogy between mental potential and fatigue and nervous potential and fatigue. 'Taking up the question of the physical basis of the latter feelings, it seems fair to suppose that their consideration will throw some light upon the mental feelings that evidently depend so closely upon them.

There are two possible sources from which considerations may come to lead us to modify this decision : first, if the consideration of physical potential and fatigue should not adequately account for mental potential and fatigue, the hypothesis of a mental expenditure would be a resource; and second, the same resource may be necessary from the consideration of the voluntary attention below.

It is said by some ${ }^{1}$ that we must be conscious of the amount of attention expended, or we would never be able to discriminate between the artificial intensity given to an object by the attention, and the real intensity of the object itself. But any such consciousness of the amount of expenditure might, evidently, arise from the degree of muscular sensation involved in the concentration of attention.

Accordingly, only the first of our three questions remains for this theory to give reply to, i.e., Are we conscious of nervous currents as they pass out of the brain, or are we conscious only of the effects of such currents in actual movements of the muscles? The question is

'Stumpf, Tonprychologie, r. p. 71, quoted by James. 
here limited to cases of reactive consciousness or reflex attention, as before.

The effect theory is not slow to answer this question in accordance with its general tenor. Its advocates challenge their opponents to produce any case of such feelings of expenditure that cannot be explained in terms of afferent sensation. The present state of the discussion is briefly indicated in a later connection. ${ }^{1}$

It is evident that this last question is not an important one in this connection. 'To establish a consciousness of the outgoing current would only give an analogy at most for a consciousness of mental outgo, and even this might be disputed by those who hold that such a nervous consciousness meets the entire case. As long as we confine ourselves to the reactive consciousness, therefore, the effect theory of attention seems to be entirely adequate from a psychological point of view.

II. Spiritual Theory of Reflex Attention. Advocates of the spiritual theory claim a direct consciousness of a mental activity in feelings of expenditure. The principal argument they urge is drawn from the apperceptive consciousness, which we have already found reason to consider an exhibition of spiritual synthesis. In brief, it is said that if there is a rational activity in involuntary attention, there must be a forceful substantial principle whose activity it is ; and it is this which we feel as activity in every attentive reaction. This argument is a metaphysical one. ${ }^{2}$ It proceeds upon the supposition that an activity is always a force, and, further, that force is a criterion of substance.

But the empirical consciousness gives no direct confirmation to this position. The relational consciousness is a consciousness of relations ; but this does not make

${ }^{1}$ Below, Chap. XV. § 2.

${ }^{2}$ Hering, however, argues the case from an experimental point of yiew, Hermann's Handbuch der Physiologie. 
necessary an element of consciousness due to a pure activity by which these relations are constituted.

This is one of the points at which the friends of the spiritnalistic hypothesis claim too much. Recent discussion has tended to purify that hypothesis of nany elements long thought essential to it. It is not in place here to be dogmatic: it is sufficient to say that in the writer's view the place to defend spiritualism is in connection with the phenomena of will. It is needless to say that the association theory which is victorious here will be found to be its most formidable rival there. But it should also be remembered that the association theory fails signally to account for the relational or apperceptive constructions of the intellect.

The distinction between what is really reflex or involuntary, in the attention, and what is voluntary, must be, if possible, clearly made. In actual fact, the voluntary act of concentration' follows so closely upon the simple conscious reaction that it may be only an abstraction we have been pursuing after all; but in this connection it may be well to remember that the feeling of expenditure was called above " hypothetical." The pursuit of it will serve, at any rate, to free us from a superstition, and give a free and open theatre for the discussion of the voluntary element in attention. It is really an open question whether the apperceptive consciousness is ever quite involuntary or reactive: we have as high an authority as Wundt holding the contrary" ; and on his view, the parallelism between the synthetic activity of relation and the forceful activity of volition holds good. There is something temptingly simple about this; but facts seem to show that there is often attention which gives us knowledge by simple reflex stimulation.

\section{§6. Conclusion on Reactive Consciousness.}

From the foregoing, the conclusion is that the reactive consciousness, per se, is simply consciousness of nervous reactions, and memnities of such reactions or of their elements. As far as there is a consciousness of

${ }_{1}$ The further attention to the loud sound which calls me out at first retlexly.

'Stumpf has receutly developed a similar view, Tonpsychologie, vol. Ir. 
self in reflex attention, it is an objective felt self, rather than a subjective, feeling, active, self. Whatever ground may be found subsequently for such an active executive self, we find no such ground here.

This conclusion is thrown into prominence by the entire group of facts of hypnotism. Here the subject is quite and entirely reactive. His consciousness of his own power of choice, exertion, initiative, is gone, and the mechanical nature of his nervous processes works up through the relational consciousness which he still has. Instead of having a suggestion from without, let us suppose him acting from simple sense-stimuli, or from memories thrown into his consciousness from within, and the whole case is plain before us. Whatever feeling of activity a hypnotized man may have, it is evidently an activity of his nervous system as it reflects the activity of the mind of some one else.

On the motor consciousness and involuntary attention, consult: Preyer, Mind of the Child, I. chaps. viII-XIV; Wundt, Menschen und Thierseele, 14te Vorl.; James, loc. cit., I. p. 434 ff., II. chap. xxIII, and (habit) I. chap. IV-V; Volkmann, Lehrbuch, \$\$ 45-48; Lotze, Medicinische Psychologie, \$§ 24-27; Warner, Physical Expression, chap.v; Sergi, Psych. Phys., liv. v. chaps. 1-3; Schneider, Mensch. Wille, chap. XI ; Ladd, Elements, part II. chap. IX. \$\$2631 ; Bain, Emotions and Will, part II. chap. I. \$ 6-8 ; Carpenter, Ment. Phys., bk. I. chap. vi. 3, chap. viII (habit), and bk. I, chap. III (attention) ; Féré, Mind, viII. pp. 210 f.; Ribot, Psychology of Attention; N. Lange, Philos. Studien, Iv ; Marillier, Le Mechanisme de l'Attention, Revue Philos., xxvII ; G. E. Müller, Theorie der sinnlichen Aufmerksamkeit; Stumpf, Tonpsychologie, 1. p. 67 ff. ; also see references given at the end of Chap. V in the author's. Senses and Intellect, and at the end of Chap. IV above.

Further Problems for Study :

Experiments upon reflex attention. 


\section{CHAPTER XIII.}

\section{STIMULI TO INVOLUNTARY MOVEMENT.}

Notion of Stimulus. An involuntary reaction in movement has already been analyzed into its three aspects or parts, i.e., stimulus, consciousness of reaction, and äctual movement. Further, the second of these elements has been reduced to the third in cases where volition does not enter. Leaving the third for discussion in connection with voluntary movement, it remains to inquire into the nature of the various stimulations which issue in conscious but involuntary reaction.

By stimulus is meant the affective experience of any kind which tends to issue in conscious motor reaction. Looked at from the side of the nervous system, it is the new element of tension, whenceever it comes, which disturbs the equilibrium outwards. And from what we already know of the nervous system, we readily see that such new elements of tension may come either from some condition of the nervous organism or from outside the system. Accordingly, stimuli to the reactive consciousness may be distinguished as organic and extraorganic.

\section{§ 1. Kinds of Motor Strmuli.}

I. Extra-organic Stimuli to Movement. The various special kinds of stimulation, as light, sound, etc., have already been sufficiently discussed, as also have the external causes of the more obscure phases of sensibility. All of these, representing, as they do, different environ- 
ing conditions, have a direct or acquired motor value. By direct motor value is meant the reactions which certain sensations occasion: reactions which seem to be inherited. And by acquired reactions is meant the movements which we come to associate habitually with certain sensational events. An example of the former is the crying of a child in pain; and of the latter, the movements of speech as they become associated with the corresponding word-sounds.

Such indirect or acquired reactions are typical, and simply indicate the gradual formation of definite paths of nervous discharge into which the energy of the stimulation is gathered. They supersede the diffused wave of effects found before such pathways are formed. In child-life this process is clearly seen in operation. The child at first uses hands, feet, and tongue together, and only gradually becomes able to gather all his energy of reaction into a definite and appropriate channel. In adult life, also, very intense stimulations cannot be held within their ordinary channels, but become diffused through many courses: note the contortions of the man undergoing torture at the hands of a dentist.

Stimuli to Reflex Reactions. All reflexes are stimulated from without: and they cover a wide range of phenomena. There is no hard and fast line between pure inherited reflexes and the acquired coördinating reflexes which come later. The stimulus to the former is simple, and calls into play a system of muscles grown together, so to speak, for a particular reaction. Such reflexes upon external stimuli occur in earliest childhood, i.e., sucking, winking, and probably the first essentials of walking-a reflex alternation of the legs, ${ }^{2}$-swallowing, etc.

1 Bain. This alternation came out very clearly in the case of my own child, but, strange to say, the movement of the legs was such as to carry the child backward. 
Suggostion as Motor Stimulus.' By suggestion is meant a great class of phenomena typified by the abrupt entrance from without into consciousness of an idea or image which becomes a part of the stream of thought and tends to produce the muscular and volitional effects which ordinarily follow upon its presence. I suggest a course of action to my friend-he may adopt it. Besides this fact of ideal suggestion, there is what may be called physiological suggestion: covering the same class of phenomena in cases where the suggestion does not attain the standing of a conscious image, but remains subconscious. It is called physiological because the nervous process, as in all cases of very faint degrees of consciousness, is largely self-acting or reflex. By physiological suggestion, therefore, is meant the bringing about of a reaction unconsciously or subconsciously by means of an extra-organic stimulus.

The clearest examples of such suggestions occur in sleep. Words spoken to the sleeper get intelligently answered. Positions given to his limbs lead to others ordinarily associated with them: the sleeper defends himself, withdraws from danger, etc., etc. The early development of the child's consciousness proceeds largely by such suggestions. Before mental images are definitely formed and subject to association, we find many motor reactions stimulated by such physiological suggestions from the environment. It may be called in child-life the pre-imitative stage, imitation being a reaction stimulated by a conscious suggestion. The process in physiological suggestion is indicated by our " motor square," in Fig. 16, $A$; sg being the suggesting element.

The physiological process involred in such reactions must lic on the border between true reflexes and ideal suggestion.

'Cf. the writer's observations upon his child, in Science, xvI (1891), pp. $113 \mathrm{ff}$. 
The stimulus to a reflex reaction is a nervous cause, and we need the word suggestion only when there is a modification in the way of adaptation or unnecessary response to the incitement. Undoubtedly, real suggestion involves the adaptive and selective function which we have seen to belong to consciousness, even in its lowest and dimmest forms, and the hypothesis, again, of one or more secondary consciousnesses found useful in other connections would help us here. There may be-Binet and Janet would say, on the strength of many alleged and so far undisputed facts, that there is-a subsidiary or split-off consciousness which acts on suggestions overlooked or unreceived by the main personal consciousness.

From physiological the child passes to sensori-motor suggestion: the type of reaction which illustrates most clearly the law of dynamogenesis already stated. ${ }^{1}$ In this case it is a sensation, a clear state of consciousness, which liberates motor energy and produces movement. Besides the inherited sensori-motor couples, which are numerous and well-marked, other reactions grow up early in life and become habitual. Of the latter, the following may be mentioned in particular.

1. Sleep-suggestions. The early surroundings and methods of inducing sleep become powerful reinforcements of the child's drowsiness, or even substitutes for it. $^{2}$

2. Food and clothing suggestions. These represent the spheres of most frequent and highly-spiced joys and sorrows, and their reactions soon take on the involuntary and yet highly purposive character which marks our adult attitudes toward dress and the table.

3. Suggestions of personality. The child shows preferences for individuals at a remarkably early age. $\mathrm{He}$ seems to learn and respond to a personal presence as a whole. Probably the voice is the first indication of his nurse's or mother's personality to which he responds, then touch, then the sight of the face.

\footnotetext{
1 Above, Chap. XII. \& 1.

${ }^{2}$ See the writer's detailed observations, loc. cit.
} 
4. Imitative suggestion. The simple imitation of movements and sounds, clearly manifested about the seventh month of life.

In ideo-motor or ideal suggestion we pass to the motor aspects of images, reproductions. And here the motor accompaniments are largely associations and follow the laws of association. As soon, further, as reproductions come up, with their suggested trains, we find the rise of will: that is, they become stimuli to the voluntary consciousness-a topic for later discussion. Yet there, is a state of conflict and hindrance among presentations which is mechanical in its issue, the attention being drawn in a reflex way. So states of vexation, divided counsel, conflicting impulse, and hasty decision against one's desire for deliberate choice. We often find ourselves drawn violently apart, precipitated through a whirl of suggested courses into a course we feel uuwilling to acknowledge as our own. This state, called by the writer deliberative suggestion, characterizes many actions of the young child before will is clearly exercised.' Figure 16, $B$, presents a diagram of sensori-motor and ideal suggestion.

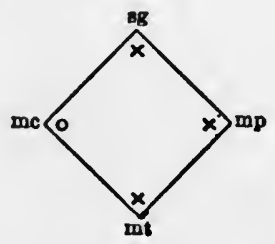

$\mathbf{A}$

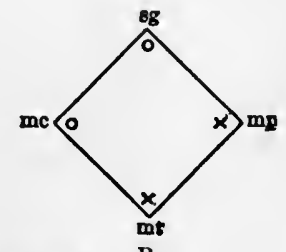

B

Fro. 16.-Suggestion.

II. Organic Stimuli to Movement. Again, the results of the former classification of the organic sources of feeling serve to cover a great area of the present topic. In general, any condition of the organism, be it active

I See the article just cited for a detailed example. 
or passive, which is sufficient to reach consciousness, tends to muscular expression, either natural or acquired. Any derangement of the digestion, respiration, or circulation, quickens or deadens muscular tone, and comes out, if not in the face, yet in the conduct of the man. The muscular feelings themselves, so large a portion of the "general sensibility," reflect direct changes in the tendency and direction of motor reactions. Diseases of the. nervous system find their diagnosis in their effects upon the muscular apparatus: paralysis means rigidity; epilepsy, convulsions; sleep, flabbiness of the muscles. The effects of organic stimulation upon the motor consciousness is best seen in conditions of pleasure and pain.

Expressive Reactions. Among direct or native reactions, an important class are called expressive: they are differentiated muscular movements which reflect uniformly various affective states of consciousness. These reactions have already been discussed above. ${ }^{1}$

Pleasure and Pain as Stimuli to Movement. Perhaps the most direct and invariable stimulus to involuntary movement is pain. And its motor force is independent, as it seems, of the intrinsic experience of which it is the tone. The motor force of a sensation of light, for example, may be in direct antagonism to the motor force: of the pain which the light causes to a diseased eye. Despair begets inaction, but the painfulness of it begets restlessness. This is only to say that the tone is an element of sensibility apart from the sensation it accompanies, and that both the one and the other have motor force.

Yet the fact that there are no experiences absolutely indifferent as respects pleasure or pain gives the motor 
aspect of them an universality and importance which must be acknowledged and provided for in any mental theory. It is a question answered often in the negative, whether any course of conduct is ever pursued without primary reference to the pleasure it will bring or the pain it will avoid. However this question may be answered later,' it may be said at this point that no line of muscular reaction is possible in which an element of motor discharge due to pleasure or pain has not entered. This must be true if the fundamental position is true that every ingoing process alters the equilibrium of the central system and modifies the direction of its outward tendency. Pleasure and pain arising from bodily states. may, therefore, be called the most general internal stimuli to the reactive consciousness.

The relation of sensuous pleasure and pain to the kinds of sensibility they accompany has been seen to be the same as the relation of ideal tone to the emotions: and the distinction in motor force between tone and sensibility proper, if made ont in one case, miglit be expected to hold in the other. In other words, if we have reason to believe that there are sensuons stimuli to the reactive conscionsness apart from pleasure and pain, we will probably find two kinds of ideal stimuli also in the voluntary life, i.e., that pictured plcasure and pain are. not the only ends of voluntary choice.

The universality of sensuous tone, already remarked, makes it difficult to find pure stimuli of any other character; yet in early child-life such stimuli do appear. The writer is convinced, from the observations referred to, that suggestion is such an original motor stimulus. That suggestion is not itself guided by the presence or absence of pleasure or pain is evident from the fact that the suggested reaction often antagonizes pleasurable or induces painful consequences. After repetition, the resulting pleasure or pain becomes the controlling influ-

\footnotetext{
' Chap. XIV. $\$ 2$.
} 
ence, "other things being equal," and so the suggested reactions are dropped, in the one case, or made intentional, in the other : but originally the suggestion seems to be sufficient unto itself.

Nature of Pleasure and Pain Reactions. The motor reactions stimulated by pleasure and pain must be carefully distinguished from similar movements which the idea or memory of pleasure or pain leads to. Such latter movements are spoken of later. The presence of sensuous tone has an immediate influence on movement, apart from the traces left in memory. And this influence may be either furthering of such movement or inhibitory of it.

We have already seen that moderate activities are generally pleasurable. It would be expected, therefore, that pain would have a deadening and quieting effect upon the muscular system: that such an effect would tend, by reducing muscular activity to a moderate amount, to alleviate the pain and induce pleasure. It may, accordingly, be said that a painful motor reaction tends to suppress itself.

Again, in cases of extreme pain, we would expect, in addition to the above, that the activities of other motor elements would reinforce the inhibitory process, i.e., draw off energy from the painful reaction. Accordingly, we find that violent pain stimulates a diffused and convulsive motor reaction.

And yet again, since pleasure accompanies moderate function, we would expect the same two considerations to operate for the continuance of a pleasurable reaction; namely, that the life-process would be furthered by the repetition of a pleasurable reaction, and by the quieting of other activities which interfere with it and dissipate its energy. Hence we may say, a pleasurable motor reaction tends to persist. 
Looked at physiologically, it is easy to see that under the action of these principles the nervous organism very early begins to adapt itself to its varied stimuli. Painful reactions are rapidly dropped, pleasuruble reactions soon become fixed and habitual, and the law of pleasure and pain becomes, for the reactive consciousncss, the principle of its life. Suppose the infant continued to hold his hand in the flame after he felt pain, or did not change his position when lying upon a pin; or suppose he withdrew from his bottle as soon as he tasted its sweetness: if he continued to live at all, it wonld be by reason of assistance from others who had learned to live on another principle.

Here, again, a sharp line is drawn between the reactive and the voluntary consciousness. 'The effect theory, found sufficient for reactive expenditure, also proves sufficient for reactive stimulation, i.e., we have not appealed so far to any agency beyond the conscionsness of physiological conditions for the incitement of muscular movement.

Motor Spontaneity. The observation of infants clearly tends to show that movement is no less original a fact than feeling. ${ }^{1}$ It is impossible to say whether all ante-natal movements are in response to feeling conditions, as claimed by some; just as it is impossible to prove that the beginning of feeling is possible only after sufficient physical organization to make motor reaction possible, as claimed by others. It is altogether probable that the two kinds of phenomena are equally original, and depend upon each other. This is certainly the case, at any rate, at the dawn of independent life. Internal conditions of the organism itself are sufficient stimuli to an endless variety of movements. Such reactions which are simply the discharges, the outbursts, of the organism, independent of definite external stimulation, are called spontaneous. So the incessant random movements of infants and the extraordinary rubber-like activity of the year-old child.

'Preyer finds spontaneous movements in the embryo chick "without the least change in the surroundings and long before the reflex excitability is present at all." Mind of the Child, trans., r. p. 201. 
The movements of infants seem to indicate greater intensity of motor feeling than is found in adults. A child's extreme restlessness is due to a high feeling of potential or readiness of discharge; and fatigue is accompanied by a correspondingly complete collapse of muscular movements. This follows from the mobility of the infant's cerebral elements before they are pressed into definite connections and systems which give them greater inertia, on the one hand, and greater general capacities for continued expenditure on the other.

Upon this superfluity of motor energy is built up the so-called play-instinct, which is not definite enough in its channels to be classed properly as an instinct. The energy of the muscles is brought under voluntary control to gratify other senses than the muscular sense itself. Educationally, play is important, as tending to give the child mobility of movement, and a sense of arrangement, form, and complex situation: it is also a valuable aid to the growth of the inventive and constructive faculty.

\section{§ 2. IMpUlse AND Instinct.}

In the foregoing section, the stimuli to the reactive consciousness have been seen to come from within or without the organism. As originating mainly within, they may be called in general impulsive, and as originating mainly without, instinctive. With such an inexact distinction for the present, the more definite inquiry into impulse and instinct may be begun.

Impulse. By an impulsive character we understand one in which activity predominates; but activity of a somewhat capricious kind. We contrast a creature of impulse with a creature of reason. And this means more than that the impulsive individual can give no adequate reason for his outbursts : it means also that 
no one else can. Impulses are essentially unreasonable to the onlooker. They are capricious in the sense that they are, to a degree, idiosyncratic.

In this case, as in so many others, the result of close analysis is only a confirmation of our ordinary definition. Looked at from the side of physiology, sensory and motor processes are such only as they are correlative and antithetic to each other. The physiological unit is an arc, a reaction. Psychologically, we find a similar state of things. At the beginning, as far as investigation can discover, there is an element of motor feeling - of going out, as well as of taking in. And this "going-out" element gets to itself, wherever we find consciousness, a kind of personality or idiosyncrasy, seen in its selective reactions, and in the kind of character which it builds up. The ribs, so to speak, of consciousness go in pairs, just as the sensor and motor nerves serve as rib-pairs in the nervous system: and taken together as pairs, they constitute, on our last analysis, the foundation of all conscious life. In dealing with sensibility, we are dealing with one side of this pair. What sensibility is, is an inscrutable mystery : it is an ultimate psychological fact. And the same is true of impulse: it is the other element in the fundamental pair.

Yet, in the way of description, we may make the following observations about impulse, in the light of what we know of physiology and of general consciousness.

1. Impulse belongs to the reactive consciousness : it does not involve deliberation and will. A deliberative character is a man who controls his impulses, that is, one who brings his will to bear effectually upon his impulses. On the other hand, very strong and varied impulses tend to overpower and paralyze the will. Impulse should therefore find its general condition in the physiology 
and psychology of the involuntary life. It follows that the end of impulse is not pictured in consciousness.

2. Impulses are never quite beyond control in normal circumstances. They are sufficiently internal and unreflex to be subject to voluntary negation. Yet their influence upon the volitional life may be very great, as appears later in the consideration of them as motives to action. In cases of long indulgence or weak resolution, their subjugation can only be indirectly accomplished; that is, by the active pursuit of other lines of activity, by which the force of the unprofitable impulse is drained off into adjacent channels.

3. The idiosyncratic character of impulse must be due largely to constitutional tendencies of individuals derived from inheritance or from peculiar conditions of life. The effects of inheritance in this particular are very marked. Nothing is so evidently inherited as emotioual temperament. This is what we would expect from the law of nervous heredity, as already stated above. And in the individual life, the growth and decay of impulse is also easily observed. Discouraging circumstances or continued illfortune may reduce a man of hopeful impulses to a prevailing pessimism and lack of interest. This characteristic individuality of impulse prevents its division into classes, and makes it impossible to formulate for single impulsive reactions any exact laws of stimulation.

4. Impulse is, therefore, internally stimulated : and cannot generally be analyzed into definite reflex elements. This is true on both the physiological and the psychological side. A physiological impulse cannot be traced directly and uniformly to a particular stimulus : it seems to be rather the outcome of what is peculiar to the central process, and to result from the growth of the system. And, on the other hand, we cannot trace impulses in consciousness to uniform psychological antecedents. They seem 
to represent the state of consciousness as a whole, apart from the theoretical worth of particular images. Impulses of fear in nervous persons are, and persist in being, quite independent of argument and persuasion. Our reasoned conclusions frequently have to fight their way through many opposing impulsive tendencies.

Yet it is generally through the presence of some definite object or image that impulses are clearly manifested. What may have been a vague feeling of unrest or disquiet turns into an impulsive motor reaction whenever it finds its appropriate object, as Jessen remarks.'

Definition of Sensuous Impulse. Accordingly, we may define sensuous impulse psychologically as the original tendency of consciousness to express itself in motor terms, as far as this tendency exists apart from particular stimulations of sense.

Kinds of Sensuous Impulse." Confining ourselves for the present to the sensuous side of impulse, we find that such tendencies are either positive or negative-toward or away from a present stimulation. The impulses following pain are away from the cause of pain, those arising from pleasure toward the source of pleasure. They do not involve, however, definite purpose, or the adoption of conscious ends. The purposive character which they have is a case, as far as psychology goes, of original adaptation.

Farther, such impulses are either furthering or inhibitory, respectively, of motor reaction. As has been seen, the effect of moderate pain is, generslly, quieting or inhibitory. Yet an important class of physical pains induce definite and violent motor agitation: these are the discomforts arising from physical lack or unsatisfied appetite.

${ }^{1}$ Prychologie, pp. 346, 347.

* Schneider's Sensationstrieb, see his Thierische Wille. 
All the animal appetites are native and their appropriate motor apparatus comes into impulsive activity. The impulses which spring from pleasurable states are uniformly furthering.

Instinct. The general word impulse was given to the more complex motor tendencies as far as they are internally initiated : similarly, complex reactions which are stimulated from the environment are called Instincts. The division between the two classes is thus a broad line of demarcation, subject to exceptions and anomalous cases on both sides. From the standpoint of common observation, two great characters seem to attach to instinct: first they are considered a matter of the original endowment of an organism, and further they are thought to exhibit the most remarkable evidence in nature of the adaptation of organisms to their living medium.

Assuming in advance that instinct is a complex motor phenomenon stimulated from without, empirical observation enables us to make the following remarks in the way of further description.

1. Like impulse, instinct belongs to the reactive consciousness and is original. This is now sufficiently understood.

2. Ordinarily, instinct is not under voluntary control. Here the case differs from the phenomenon of impulse.

3. Instincts are as a rule definite and uniform: they lack the idiosyncratic and individual variations of impulse.

4. Instincts are correlated with definite stimulation, to which they afford reflex reaction.

In saying that instincts are reflex, we bring to mind all the characteristics of such reactions: their mechanical nature, as fixed types of nervous process, their irresistibleness as phenomena of consciousness, their particular forms, as belonging to distinct animal species. They represent the consolidated nervous structure which is transmitted by inheritance, and the low form of con- 
sciousness which has not character enough to be impulsive.

In saying that they are reflex, it is further meant that instincts do not carry cousciousness of the effects which they work. [ The hen, when she first "sits" on her nest, has no picture of her future brood, and no purpose to hatch her dozen eggs. In saying she has an instinct to "sit," we mean that when her organic condition (warmtli, etc.) is so adjusted to the environment (nest, eggs, etc.) that hatching will ensue, she sits by a necessity of her reflex nervous organism. [ So we cannot say that migrating birds have a picture of the country to which they fly for the first time, or an anticipation of the congenial warmth of a southern clime:- all we can say is that, atmospheric and other conditions acting as stimuli, the bird's migratory instinct shows itself as an appropriate motor reaction.

Complexity of Instinct.' But the simple concept of reflex reaction needs some modification in view of the marvellous complexity of observed instincts. If the purposive adaptations of the organism were limited to a single reflex arc, i.e., to a sense-stimulation and a muscular movement in reaction, the life of the animal world would be cut off at a low level of development. The adaptation to its environment, on the part of the nervous system, must gain this complexity in two ways : first, by a coördination of muscular elements in a single group for a common end-what we may call a coexisting complexity -or, second, a union of successive motor reactions in a. dependent series for a common end-what we may call a serial complexity. Both of these are realized in animal instinct. The bird's nest-building involves both the simultaneous performance of many muscular reactions

' On both the complexity and the reflex nature of instinct, see Spencer, Prychology, §194. 
and the long succession of movements in flight, etc., from day to day, which in voluntary life we call the employ. ment of means to end.

Apart from the original fact of adaptation, this serial complexity, extending, as it often does, over great periods of the creature's life, is the most extraordinary aspect of animal instinct. The entire life of some species of birds is a round of successive instinctive adaptations to atmospheric and temperature conditions. And the complexity is often a remarkable simnlation, to put it mildly, of conscious rationality. Witness the social and political life of ants and bees. How it is to be explained biologically, it is not ours to inquire; psychologically, there is nothing to explain. An instinct in consciousness is a blind outward tendency in response to sense-stimulation. 'To put anything more into it would be to call unwarrantably upon a foreign science for more data, or on philosophy for wider interpretation.

As Romanes ${ }^{1}$ makes clear, the purely reflex theory of instinct, as held by Mr. Spencer, overlooks the part played by purposive conscionsness and intelligence in the formation of instinct; and in this he is supported by the pronounced evolutionist Schneider, ${ }^{2}$ who holds that there is always a conscious state through which the instinctive reflex works. There is undoubtedly a class of phenomena here which neither the hypothesis of "nervous reflexes" nor that of "lapsed intelligence" is sufficient alone to explain : phenomena of the adaptation of the nervons system to new reactions through consciousness which is yet not will. From study of the child mind the writer has elsewhere endeavored ${ }^{3}$ to justify the use of the word suggestion exclusively for this class of facts : the word in this usage meaning the modification of a reflex reaction through the conscious state which links together its sensor and motor branches. Such a conception unifies the two ways in which we may suppose instinct to have arisen : namely, first, by a modification of nervous reflexes through suggestion (what the writer calls "physiological " and "imitative" suggestion), and second, the lapsing of intelligent voluntary reactions into secondary-automatic, and finally into suggestive reactions ("sensori- or ideo-motor suggestion"). On the

1 Mental Evolution in Animals, p. 266 ff.

- Der Thierische Wille, chap. III.

${ }^{3}$ Above, $\$ 1$ of the present chapter: 
organic side, these two laws of the rise of instinct represent " "ipward" and "downward" growth, respectively, of the nerrous system."

Definition of Animal Instinct. From the point of view of consciousness, instincts are original tendencies of consciousness to express itself in motor terms in response to definite but generally complex stimulations of sense; i.e., they are inherited motor intuitions.

Both this definition and that of impulse must be held subject to the artificial precision which exact statement unavoidably entails. In consciousness the distinction between them is important for its bearings in the voluntary life : for it may be held that impulse is ethically available, while instinct is not. On the physiological side, the distinction seems to be only a relative one.

Yet even from the side of consciousness, the word instinct is often used to cover impulse, especially the adjective instinctive, as synonymous with the word original. Prof. James adopts this usage, and it leads him to an exaggeration of the reflex side of impulse, at the same time that he tones down the meaning of reflex by a correspondingly strong statement of the variability of instinct proper. Preyer's view, with which the above definitions are in substantial accord, is in better harmony both with the psychology of impulse and with the physiology of instinct.

${ }^{1}$ Lewes and Schneider (Thier. Wille, p. 188) advocate the view that instinct arises exclusively from " lapsed intelligence." Romanes, following Darwin, recognizes both the principles of the text; the former, under the phrase "law of natural selection," being called by him the " primary," and that of lapsed intelligence the " secondary," prin. ciple of the origin of instinct. His is probably the most adequate treatment of the question yet written, Mental Evolution in Animals, chaps. $x I-x v i I$. I am unable, however, to agree with him in drawing a sharp line between reflex and instinctive action on the basis of differences in the "mental elements" involved; i.e., he holds that reflex action involves sensation only, while iustinct involves perception, ibid., p. 160. On the contrary, it seems clear that each may involve either. It is difflicult to assign, for example, nny perception to the migrating instinct, or to confine such a reflex as the closing of the eyes before a foreign body to sensation. On the origin and development of particular human instincts, see Preyer, Mind of the Child, I. chap. XI (trans.). 
Variability of Instinct.' The experience theory of instinct is further strengthened by the fact of variability, possible modification, or entire loss of an instinct by reason of changes in the stimulating conditions. Recent observations have established this point beyond question. The child loses the power of sucking after he has been weaned; and if he relearn it, it must be by a gradual process. Birds in confinement lose the nestbuilding instinct. Bees will so modify their hive structure as to overcome new and quite artificial obstacles, while still retaining the architectural principle essential to economy of material. We accordingly reach a broad class of phenomena which seem to lie on the border-line between impulse and instinct, as now defined, and which tend to bring unity into this phase of conscious life. The facts may be gathered under the following points.

1. Decay of Instinct from Disuse: a principle which explains itself. Physiologically it means the encroachment of nerrous combinations which are used upon the material or connections of such unused apparatus: the result being a readjustment of elements in a way which destroys the former instinctive reaction.

2. Modification of Instinct from Imperfect Adjustment. This means the reversion of reflex coördinations to a less complex type. The bird that has lost the nest-building instinct may still retain the egg-laying and mating instincts, although in a wild state it is difficult to draw any line of division between them. The adaptation of the reaction to that degree and kind of stimulus actually present is wonderful, but still a fact. It is probable that this modification of instinct is due in part to the influence of memories of earlier experiences, the present elements of stimulation working by help of reinforce-

${ }^{1}$ Cf. Romanes, loc. cit., pp. 167-203. 
ment from their own memories. In this way the elements essential for a present reaction are emphasized. Initative suggestions tend, in the same way, to modify instiucts. Voluntary selection, also, breaks up instincts, until in many cases only the impulses remain, so to speak, instinctive.

3. Natural Exhaustion of Instincts. Many instinctive reactions naturally spend themselves and die away. Thus the infant's sucking instinct, the gregarious instinct in some, the bashful instinct in others. In many cases the instinct of modesty seems to disappear altogether as life advances. So the fires of physical enjoyment and the instinctive enthusiasms of youth fade and perish together. Such instincts represent phases merely in the life-history of the physical and mental organism.

A detailed enumeration of animal impulses and instincts is required for fulness of descriptive treatment, but it has none but illustrative interest. Preyer ${ }^{2}$ and Schneider ${ }^{3}$ have endeavored such a classification of original reactions, and James ${ }^{4}$ follows them. We may dispense with such a list ; only remembering that in impulse we have a phenomenon as original, as far as psychological analysis goes, as sensation, and that impulses, like sensations, seem to be qualitatively distinct, while still one in their nature as impulse.

\section{§3. Affective Nature of all Stimuli to Movejient.}

Affects. In the foregoing notice of different classes of stimuli, the fact has been assumed that they are all phenomena of feeling. We feel the force, the motor worth, of a suggestion, a pain, an impulse. An idea simply as an idea-if such could be realized-might not react in movement; but the simple presence of an

${ }^{1} \mathrm{Cf}$. Fortlage, Psychologische Vorträge, p. $229 \mathrm{f}$.

The Senses and the Will, part Ir.

${ }^{3}$ Der Menschliche Wille, Theil II.

Loc. cit., II. 403-441. 
idea in consciousness is itself a feeling, and only in as far as it affects us does it move us. ${ }^{1}$

We may accordingly apply the term affects to all stimuli to involuntary movement. When I am affected, I am moved through my own inner state of sensibility. And such affects also figure, "as shall appear, in the voluntary consciousness as well; but there they stand in contrast with another great class of stimulations, which together with them constitute motives. Affects, therefore, are the feeling antecedents of involuntary movements; as motives, including affects, are the inner antecedents of acts of will.

This use of the word affect in a peculiar sense seems justified by the meaning given to it, even from a conservative view of the right to assign new meanings. The phrase "sensuous impulse," however harmless intrinsically, has become a snare and reproach to the new idealist. Green's limitation of motive to rational end is arbitrary and unwise. Motive means anything that appeals to the will; not pictured ends alone, but often the vaguest analogies of feeling: and in this category are included, not impulses alone, but all the various stimulations mentioned above. The rôle of the affect, at any rate, is sufficiently great in the sequel, whatever may be said about its right to be baptized with a name.

Division of Affects. From the above description of motor stimuli we may conclude that involuntary movement, when not spontaneous nor simply reflex, results from one or more of the causes in the following table:

$$
\text { Stimuli. }\left\{\begin{array}{l}
\text { Pleasure and pain. } \\
\text { Suggestion. } \\
\text { Impulse. } \\
\text { Instinct. }
\end{array}\right.
$$

Speculative analysis may reduce instinct to impulse and suggestion: impulse may be expressed in terms of pleasurepain and stggestion. These two, however, pleasure-pain and

${ }^{1}$ On the affective nature of impulse, see Lotze, Microcosmus, p. 255. 
suggestion, remain outstanding, both in psychology and in ethies. Under pleasure and pain, also, must be understood all states of feeling considered as pure feeling-such as physical and emotional excitement, passion, etc., the consequences of which become more apparent in the next chapter.

On suggestion and impulse, consult, in general: Perez, l'Education des le Berceau, part IV. chap. 5; Bcaunis, Sensations Internes, chaps. II-VI ; Baldwin, Science, vol. xvII (1891), pp. 113 ff.; Wundt, Phys. Psych., 3d ed., II. pp. 404 ff.; Rabier, Psychologie, chap. xxxvi ; Martineau, Types of Ethical Theory, II. bk. I. chaps. 5-7; Schueider, Thier. Wille, Iv and v, and Mensch. Wille, chap. xII; Bain, Emotions and Will, part II. chap. 1; Drbal, Lehrbuch, \$129-34 ; Garnier, Traite, bk. Iv. chaps. 1-4; Wundt, Gemüths bewegungen, Philos. Studien, vi, Heft 3; Preyer, Mind of the Child, I. pp. 157-215; Volkmann, Lehrbuch, I.pp. 321 f.; Lotze, Microcosmus, pp. $254 \mathrm{f}$.; Radestock, Habit and its Importance in Education; Guyau, Education and Heredity.

On hypnotic suggestion: James, loc. cit., II. chap. xxvII ; Moll, Hypnotism; Binet and Féré, Animal Magnetism ; Bernheim, Suggestive Therapeutics; numerous articles in back volumes of the Revue Philosophique and the Revue de l'Hypnotisme.

On instinct : George, Lehrbuch d. Psychologie, p. 167 ff. ; Bastian, The Brain as an Organ of Mind, chap. xIV ; Fortlage, Psychol. Vortrage, vi ; Lewes, Problems of Life and Mind, 3d Series, Prob. III. chap. 1 ; Preyer, Mind of the Child (trans.), I. chap. $\mathbf{x 1}$; James, loc. cit., II. chap. XXIV; Volkmann, Lehrbuch, \$146; Romanes, Ment. Evol. in Animals, chaps. XII-xvill ; Morgan, Animal Life and Intelligence, chap. XI ; Schneider, Menschliche Wille, Theil II, and Thierische Wille, III; Spencer, Psychology, II. part Iv. chap. 5; Perez, First Three Years, chap. IV ; Bascom, Comparative Psychology, pp. 147 ff. ; Joly, l'Instinct.

Further Problems for Study :

Play-instinct ;

Normal and hypnotic suggestion;

Muscular movements in sleep ;

Infants' movements. 


\section{MOTOR ASPECTS OF IDEAL FEELING.}

\section{CHAPTER XIV.}

\section{STIMULI TO VOLUNTARY MOVEMENT.}

The Voluntary Motor Consciousness. The general analysis already found convenient for the reactive consciousness holds for the voluntary. We find that in all cases of intended bodily movement there is, first, a reason why we will the reaction; second, the actual decision or act of will; and third, the resulting movement. All the " reasons why," taken together, constitute stimuli to voluntary movement, and they may be considered first.

Farther, we may assume, for the present, that the laws of the stimulation of voluntary muscular movement are the first and most essential application of the laws of voluntary activity in general. Whatever the will does, it does by the leverage, so to speak, of muscles: will can only express itself by muscular reaction. So what is true of voluntary movement is, in the main, true of volition generally.

\section{§1. General Stimuli.}

I. Interest in an Object. The most evident characteristic of intentional action is that something is intended, i.e., that a presentation of some kind is set before consciousness. The notion of an end foreseen, which we found absent in instinct and impulse, and undefined in ethical feeling, here becomes explicit. Psychology finds 
here, in common phraseology, one of its safest distinctions.

Yet it is easy to see that an object thus presented or apperceived must carry some interest in order to be pursued. I will to move my leg, either that I may walk - my present interest; or that I may relieve a strainalso my present interest. Some degree of present emotional interest, therefore, may be said to be the most general stimulus to volition.'

As the young child's earliest interests are its crying physical needs, it is probable that voluntary movement takes its rise in the adjustment of spontaneous and reflex movements to varying conditions, for the satisfaction of appetite. As a fact, we find the random movements of the infant very soon taking on the character of tentative voluntary explorations. These nascent efforts and their reverses gradually give rise to well-formed beliefs in points of objective reality, through the successful repetition of series of muscular sensations."

Belief, then, confirms interest as motor stimulus. So in adult life, also, interest is no longer a sufficient stimulus to volition; strong belief is necessary to rouse us to action.

II. Emotional Excitement. Apart from the impulses upon which emotions are built up, the simple fact of ex-

1 Jessen points out the need of preliminary interest to serve as a basis of permanent desire (Leidenschaft), loc. cit., pp. 293 and 335. James interprets the volitional stimulus too much in terms of the present consciousness, in limiting moving interest to "that which possesses the attention," loc. cit., II. p. 559. The deeper abiding interests seldom come into consciousness : and when their influence is grentest they do not possess the attention. The conflicting elements of present consciousness rise and fall together with the ground-swell, so to speak, of permanent habitual desire-the influence of what I have called above " affects."

"Secondary cocflcient" of exteraal reality, above, Chap. VII. $\$ 4$. 
citement stirs the will. Considerations lose their proper balance and coördination to an excited man. His emotion puts him in a general state of willing-principally physical-and objects presented are magnified or otherwise distorted. ${ }^{1}$

An interesting case of the motive force of excitement is seen in the gambling mania in all its forms, betting, gaming, horse-racing, etc. There seems to be pleasure in the alternating phases of hope and fear, with the tremendous nervous agitation which it works up when long continued. It is tinged, also, with the emotion of self-interest, but not strongly enough to make this the sufficient motive consideration.

III. Suggestion. The effect of a simple sensation as suggesting an appropriate involuntary reaction has been pointed out : the same influence from ideas is to a degree stimulating to the will. In the absence of other considerations and aims, the simple presence of an idea of movement appeals with some strength for recognition. This is ideo-motor suggestion. Perhaps the best illustration is found in persistent imitation of movements by infants." It has a wider influence, however, through the associated elements which cluster around a suggestion. The wider the range of association which a suggested action takes on, the more interests it is likely to. involve, and the stronger it becomes as a moving force. Suggestion may then be said to work by a law of associated interests.

The force of simple suggestion upon volition is seen

\footnotetext{
1 Professor Bain admits this influence as an exception to the general law that the will is moved only by motives of pleasure and pain. That is, he differs from the view in the text by ruling out of the category " motive," influences which do not work either directly or indirectly through ideas. Emotions and Will, pp. 380-81. Mental excitement, he says, works simply by facilitating motor diffusion. Ibid., p. 311.

" See my article already quoted from, Science, vol. xvII (1891), p. 116.
} 
in the many sudden impulses we have toward freaks and uureasonable actions, such as throwing ourselves from high places, attacking strangers, returning to repeat actions we know we have already done, etc. ; so also the attractiveness of the unpleasant, loathsome, monstrous. From these outgoing tendencies which are only unreasonable, suggestions shade up through many stages into fixed ideas, hypnotic illusions, permanent delusions, and more positively pathological conditions of will. ${ }^{2}$

IV. Ideal Pleasure and Pain. Movements which have no ulterior reference, which are themselves the presented objects of will, are largely incited by present pleasure or pain. So also the attention is voluntarily withdrawn from painful conditions and given where its concentration is pleasurable. But in ordinary circumstances this hedonic incitement is not itself the pictured object. It is only later, when consciousness becomes reflective, that hedonic indulgence becomes the set aim of desire and voluntary movement.

Affects as Stimuli to Voluntary Movement. A closer examination of the influences now found to bear on voluntary movement brings out the fact that they are farther and explicit expressions of the influences already found to effect involuntary reaction. The general law that sense-modifications tend to pass off in motor reactions bears right up into the voluntary sphere. Suggestion which produces involuntary movement tends to produce voluntary : so of pleasure and pain, emotion, impulse. The psychology which separates volition from reaction so sharply as to deny any influence upon the will to other stimuli than pictured ideas, is false. The conditions back of an act of choice are never limited to the alternatives between which the choice is made.

' Cf. Ribot, Diseases of Will. 
There is beneath it all a dumb unexpressed mass of affects-organic partially-felt tendencies outwards, which give coloring to the whole process. A decision made at night is reversed in the morning when no new information has been received. "A trifling physical accident will distort vision, arouse emotion, and reverse decision. This fact, that our most abstract acts of volition are strongly influenced by subconscious affective conditions, is only beginning to have the recognition it deserves.

In other words, the ideal stimuli do not supersede the sensuous entirely : both persist, even in cases where both are subordinated to represented ends. With all such representation in idea, there is also a body of stimulation, both sensuous and ideal, not thus represented, which colors the representations and modifies their motive force. ${ }^{1}$

\section{§ 2. Special Stimulus to Volition: Desire.}

Apart from the more general influences already described, we find at the basis of all voluntary movement the great fact of desire. Understanding the term as synonymous with wish-as the words are popularly used-our conception will grow more exact as we proceed.

Impulse as Basis of Desire. The remarks already made about sensuous impulse lead to an inquiry as to the ground of the attracting and repelling force inherent in certain emotions. Are there original intellectual impulses accompanying and carrying forward the apperceptive processes, as there are physical impulses preserving and furthering the physical life? This question may be answered confidently in the affirmative, as the following positions already justified lead us to believe.

' Prof. Bain urges this truth, Emotions and Will, Will, chap. vi. 
1. The interest of exploration-the infant's attention -is an ever-active, restless, changing thing. It does not a wait a law of successive stimulation, but its nature seems to consist in impulsive activity, just as spontaneous movement is the unsolicited exhibition of physical vitality.

2. In the intellectual feelings we find a demand for consistency of experience and free function analogous to the demand in sensational experience for the consistency which induces belief in the permanent external existence of the world : and one seems to be as original and impulsive as the other.

3. In the conceptual emotions, esthetic and ethical impulses seem to be presupposed. We are impelled toward the right and away from the wrong-toward the beautiful and away from the ugly. And since these emotions are dependent upon the free conceptual construction of ideals, away from the limits set by experience, the most reasonable explanation is found in the hypothesis of intellectual impulse.

4. The analogy from the distinction between sensuous and ideal pleasure and pain supports a similar distinction between sensuous and ideal impulse. We found that ideal pleasure and pain arise as soon as the intellectual process begins to be explicit; i.e., all processes higher than perception have ideal tone. So ideal impulse appears at the same stage in mental development. The impulses involved in expressive emotion are still sensuous: many of them are inherited as nervous coordinations. So is the sympathetic impulse, as far as it attaches to simple presentations of suffering or joy. It ouly becomes ideal when it is taken up into the conceptual process and becomes the basis of social, ethical, or esthetic feeling, and then we find new outreaches of impulse. 
The question of "unity of composition" here confronts us for the first time on the active side of the mental life. It should be clearly understood that "composition" will not be made out until all activity is reduced to reflex nervous reaction. So if sensuous impulse be so reduced, the next step is the reduction of ideal impulse to sensuous. Psychologically, we encounter the difficulties already stated. They may be overcome if it can be shown that volition is no more than the felt side of a compound reflex. And in that case a presumption would be raised from the active side against the cogency of the arguments already urged in opposition to the "composition" theory, from the point of view of apperception."

Holding to original intellectual impulses here, however, we do not exclude any single philosophical explanation of mental activity as a whole. Any hypothesis which will bind physical and mental impulse together, as exhibiting a common energy, is pertinent, as we had occasion to say in speaking of lower and higher pleasure and pain. The fact that physical and mental impulses support and further each other, as physical and mental pleasures and pains do, seems to invite some such philosophical hypothesis.

Kinds of Intellectual Impulse: Appetence. In accordance with what is said above, there are several kinds of intellectual impulse which lie at the bottom of the earlier classification of the emotions: logical impulse; self-impulses, seen in ambition, vain-glory, self-depreciation; sympathetic impulses, seen in generosity, self-denial, impulse to rescue, bravery for others, etc.; impulses for ideals, of truth, the good, and the beautiful. As terminating on particular classes of objects, such impulses are often called appetences. ${ }^{2}$

It now appears that the founding of belief, in its various forms, upon impulse was just. The kinds of impulse now

1 Senses and Intellect, chap. vir. \$ 4.

'Volkmann divides desires into two classes, according as they take their rise in higher or lower impulse : see his historical note, Lehrbuch, pp. 429-30. Paulhan, who makes impulses (tendances) the fundamentals of mind, divides them into personal (egoistic), social (sympathetic), and ideal; L'Activité mentale et les Éléments de l'Esprit, pp. $141 \mathrm{f}$. The usual ethical division is into egoistic, altruistic, and mixed. 
appear to yield the different coefficients of reality, i.e., sensuous impulse, the sensible coefficient; logical impulse, the logical coefficient; ethical and xsthetic imputses, their respective coefficients. The sympathetic and selfish impulses play between the sensible on one hand and the ethical on the other, for which we have found psychological reason in their rise and development.

The reduction of all impulse to one, that of self-conservation or self-preservation, has no place in psychology. 'To say that all impulse, or all pleasure, tends to the development of self or the race (Vervollkommnungstrieb ') may be true, but it has no meaning for the impulsive consciousness itself. Schneider's classification, given above as the basis of his division of feelings, serves such a purpose of reduction from the evolution standpoint. ${ }^{2}$

Desire and its Objects. The impulsive basis of desire, however, is not the whole. Intellectual impulse is a directed impulse, an impulse conscious of the object of its satisfaction. This objective reference it is that distinguishes desire from centrally-initiated reactions generally. The distinction is seen clearly in certain experiences of restless impulsireness which we feel when there is no definite object of desire. Restlessness, both mental and physical, tends to pass off in diffused accidental channels. The shifting, aimless, often destructive, muscular movements of the nervous dyspeptic find their counterpart in similar movements of his attention and emotions. But when this outward tendency is chained down to a single outlet clearly pictured in consciousness, we have desire.

Intellectual impulse represents the more permanent massive outward tendencies of our nature, of which desires are the tangible and definite expression: just as passion stands for the underlying capacity for strong emotion. The qualification "intellectual" is indicative

${ }^{1}$ Braubach, Prychologie des Gefühls, p. 28, and the new idealists generally.

Thierische Wille, p. 48. 
only of its reference to ideal objects as opposed to physical, and must not be allowed to conceal the essential affective character of the impulse itself. ${ }^{1}$

The object of desire is, therefore, that after which desire reaches out: and these objects are innumerable. In general, any presentation whatever that arouses an impulsive movement of consciousness becomes by that fact the object of desire.

Rise of Desire. The first clear cases of desire in the child express themselves by movements of the hands in grasping after objects seen. As soon as there is attention, giving a clear visual presentation of an object, we find impulsive muscular reactions directed toward it, at first in an excessively crude fashion, but becoming rapidly refined. The writer found, in experiments with his own child, ${ }^{2}$ that the vain grasping at distant objects which prevailed in a lessening degree up to the sixth month of life tended to disappear in the two subsequent months. During the eighth month, the child would not grasp at colored objects more than 16 inches distant, her reaching distance being 10 to 12 inches. This training of desire is evidently an association of muscular (arm) sensations with visual experiences of distance. It is, therefore, probably safe to say that desire takes its rise in visual suggestion and develops under its lead. The earlier feelings of lack and need springing from appetite are vague and organic, and cannot be called desires: they have no conscious pictured objects.

Desire and its Tone. The hedonic coloring of desire is always a state of pain, especially when the impulsive tendency is intense or long restrained. It begins with

1 The Germans have a distinct word (Leidenschaft) to distinguish these tendencies from the simple impulse (Trieb).

* See Science, xvr (1890), p. 247. 
a state of uneasiness or restlessness. The basis of desire, like that of appetite, is a functional need: this state of need or lack is in itself painful, and its gratification pleasurable. But both the removal of the pain and the gaining of the pleasure are conditioned upon the presence of the object upon which the function in question is legitimately exercised. For example, in hunger the lack in the nutritive function is felt as pain; the function is brought into exercise by its appropriate object, food; and the exercise of the function is pleasurable. So with the student, the lack of mental occupancy is painful, the pain is relieved by securing an appropriate subject of application, and the function thus established gives pleasure.

Originally, therefore, the hedonic coloring of the satisfaction of desire is purely an accompaniment, not in any sense the object of the desire; and this for several reasons. First, it is equally present in instinct, sensuous impulse, and emotion, when there is no conscious object.' As has already been seen, the elevation of pleasure and pain in these cases, as in the case of ethical ends, to the rank of object sought, is a distinct confusion of objective, or teleological, with subjective ends. Second, the stimulation of rolition by suggestion, as pointed out above, shows that consciousness may react directly upon ideas presented regardless of hedonic consequences. Third, the approximate neutrality of actions impelled by certain desires and emotions proves that pleasure is not their object. The desire for excitement, for example, grows intense in circumstances in which it is open to discussion to the man himself whether the actual excitement is pleasurable or painful. Such emotional states are not absolutely indifferent, but sometimes pleasurable, sometimes painful: they show that the intensity of

' See Hoffding, Outlines, p. 324. 
desire is altogether out of proportion to the intensity of the hedonic result. ${ }^{2}$ Fourth, the principal increment of pleasure or decrement of pain is not secured by acting but while acting. When the act is done, the pleasure falls off. It is, therefore, the accompaniment of function, achievement, not of possession. Whatever the object be, it only serves a hedonic purpose as long as it remains the end of pursuit. This we found to be conspicuously the case with ethical desire and the enjoyment of doing good. Fifth, when the pleasure to be enjoyed enters as an object, it gives a new and involved aspect to desire. ${ }^{2}$ The two desires often run parallel, but often also conflict. I can distinguish my desire for the pleasure of giving to a beggar from my desire that the benefit may accrue to the beggar, and find myself deciding between them. To deny the former is to deny myself an indulgence: to deny the latter is to deny the beggar a good.

In order to maintain that pleasure is always the end of desire, it would have to be shown that what we call the object has been so often the means of getting us pleasure, that we have come to take it for end without further thought. ${ }^{3}$ This would mean that while pleasure was originally the conscious end of volition, it has become so habitual that consciousness pursues the means with none but tacit reference to the real end. This is disproved directly by fact. Observations of children at the period when volition is arising show that the first stages of volition deal most directly with objects; that the child only learns by degrees to manipulate objects in order to

'Sidgwick maintains that this is especially true of sympathy and. benevolence. Methods, pp. 45-48.

' Noticed by Sidgwick, loc. cit., p. 48.

${ }^{3}$ So Bain, Emotions and Will, pp. 438-39, Sully, Outlines, p. 581, and the evolutionists, i.e., Schneider, who makes instinct a case of this transition on a larger scale, Thier. Wille, p. 124. 
increase or lengthen pleasure, i.e., learns that he can modify his natural reactions and subordinate them to the pursuit of the pleasure which they have incidentally afforded him.

'The infant's appetites aro at first directed to objects which satisfy: he drinks and casts his bottle energetically from him. After some eight or nine months, he begins to dally with his bottle, to stop awhile and return again, to continne after his appetite is satisfied; and in the child of two years and older, the pleasure of eating has clearly superseded the simple desire for food, and has become itself an object of pursuit.

This passage from simple desire for an object which satisfies to desire for the satisfaction itself is, however, so universal that the hedonist has much evidence in his favor if the adult reflective consciousness alone be analyzed. It must be admitted that personal happiness is an insidious and most powerful motive with us all. We grow to identify the good with the amount of happiness it brings to us. We go to church because we enjoy the sermon. We do not attend a party gathering because we find it pleasanter not to be too positive in our politics. Although we do not always intend it, and although the higher course is often clear before us, on equal standing as a motive, yet it is probable that we make no deliberate decisions whatever in which our own happiness has not been a factor of influence.

The real order of things is, therefore, just the reverse of what the hedonists tell us. Instead of beginning by the pursuit of pleasure, and ending by pursuing what was earlier the means to pleasure; we begin by pursuing an object, and end by degrading this primary object to an artificial position, as means to pleasure, or as a cumpetitor with pleusure for the dignity of being pursued." Volkmann ${ }^{2}$ turns the dictum

1 The subject is ably discussed by James, loc. cit., II. pp. 549-59: see also the references he gives on p. 558 .

Lehrbuch, Ir. p. 424 ; Spinoza also said the same thing long before, Eth. III. 9, Schol. 
nihil appetimus, nisi sub specie boni, by saying that we do not desire a thing because it is good (pleasurable), but it is good because we desire it-a law which the political economist is familiar with on a large scale.

Coefficient of the Desirable. A further question has reference to the attribute or quality of an image which makes it the object of desire. Why is it that there is an impulsive tendency to or from certain presentations? The answer requires a closer analysis of both the mental and the physical conditions involved.

On the mental side, it is well to remember that the various coefficients of belief are found in the satisfying quality of various mental experiences. In desire the demands for satisfaction become explicit, and the presented objects come to have value and satisfying reality according as they afford fit termini for reaction. The reproduction of such an object suggests its appropriate satisfactions, but the representation is wanting in body, reality, coefficient. Here, then, is one attribute of an imaged object of desire, i.e., the suggestion it gives of satisfactions which it does not bring.

It should be noticed that this fact is not limited to the absence of the sensational coefficient, the absence of sensereality: but extends to all kinds of objective representation. I desire not only food and drink, but wealth, fame, goodness. The image I have of any one of these does not meet my dernands upon it as the corresponding reality would. Volkmann contends ${ }^{1}$ that desire is a feeling of relative tension (wachsender Spannungsgrad) among presentations, and the end of desire is a clear unarrested presentation: an object. is not the end, he says, for then no desires could ever be satisfied. In reply, it may be said, an image simply is not the end, for then all desires could be satisfied. An object is the end in the sense that the reality of the object is the end-all that the object means to us in the way of satisfactions.

${ }^{1}$ Lehrbuch, II. pp. 397-98, also 407. See his exhaustive note of historical views, ibid., pp. 400-404. 
Further, what are these suggestions? What form do they take? Evidently the form that all suggestions take: motor form. They tend to pass off in the channels of action appropriate to the kind of satisfaction for which they stand. Now either the imaged object is sufficiently real in its connections to cause motor reactions, in which case desire is, partially at least, satisfied; or it is only competent to give what Ward calls "incipient action," i.e., a tendency to react which is held in check by the consciousness of the object's unreality. In this latter case, there is continued desire and a second element is reached, i.e., an incipient motor reaction which the imaged object stimulates but does not discharge.

These two aspects of desire are equally important. And on closer view, we see that they stand in the case of physical desire for the twofold criteria of objective reality with which we are now familiar. These criteria were seen to be, first, present satisfying quality; and second, liability to reproduction at the terminus of a voluntary muscular series. Now desire, as appears above, arises when an image excites these criteria without satisfying them, i.e., suggests satisfaction without giving it, and stimulates a muscular series without providing it a terminus. Or put as a single formula, we may say that an image is desired when it suggests satisfactions which are neither immediately present nor available by volition."

Physical Basis of Desire. The conception of the physical process underlying desire must await the conception of the processes which underlie the perception of the different kinds of reality. If the sensational reality of an object reflects itself in consciousness through a certain brain-process, then the idea of that object would rest upon a process lacking the peculiar element which

In the intensity and rhythm of desire, see Volkmann, loc. cit., pp. 413-16. 
stood for reality. The motor outlet in the two cases is the same : for the incipient reaction is the ordinary reaction which the object in question calls forth, except that it is incipient. Further, the seat of the idea communicates with the seat of the associated sensations which constitute the usual gratification, and these again with the seat of their legitimate motor reactions now incipiently stimulated. For example, I desire an apple: the picture in my book ( $s p$, Fig. 17) stimulates the muscu-

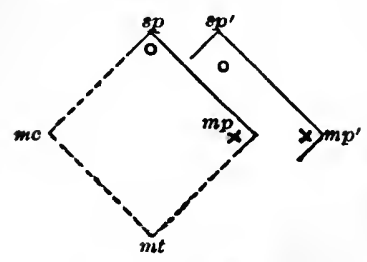

Fig. 17.-Desire. lar and glandular movements of mouth and tongue $(m p)$, and also arouses incipient sensations of taste, smell, pressure, etc., which in turn become suggestions ( $s p$ ) to new motor reactions $\left(m p^{\prime}\right)$ : but all are so faint that the remaining elements of the "motor square" ( $m t$ and $m c$ ) are not brought to fact. Physiologically, therefore, desire is the brewing of a motor storm : the beginning of what is to be when the discharge has gathered its full force in the presence of the real object.

As would be expected, motor reaction of any kind is usually a great relief in cases of intense desire and longing. The pent-up energy of the stimulated reactions is drained off into other channels. That such activity, however, does not completely satisfy desire is sufficient proof that the motor impulse is rather the means of securing the reality which gives satisfaction, than the satisfaction itself.

Aversion. In strong aversion, the mental conditions are somewhat more complex, inasmuch as strongest aversion is aroused, not when the object is merely pictured, but when it is actually at hand. This latter form of aversion is really impulse, and is much more largely a matter of the reactive than of the voluntary consciousness. As such it has been noticed under impulse. ${ }^{1}$ As

\footnotetext{
${ }^{1}$ Above, Chap. XIV. §2.
} 
accompanying representative states, it seems to be essentially the same as desire, except that it accompanies suggestions of pain rather than of pleasure, aud its motor instigation is to movements away from the object rather than toward it.

\section{§3. Voluntary Movement and Habit.}

The tendency of desire to become fixed and permanent has already been adverted to. The influence of desire upon will becomes gradually less vividly conscions and more uniform. In our adult life a few great leading principles of action are influential ; our simple desires are subordinated to our life aims, and tolerated because they advance them. Great preferences thus come to characterize a man-preferences already seen to enter in giving direction to the flow of his constructive thought ${ }^{1}$ and feeling. The law of habit, therefore, here as elsewhere, reduces the vivid to the faint, the difficult to the easy, the unusual to the customary. The content of the voluntary consciousness falls back into the reactive consciousness.

There is, therefore, a constant play both upward and downward. Stimuli to involuntary movement get taken up by rolition, and utilized to aid the law of conscious preference : nature becomes an instrument in the hand of will. But will, by using, fixes and unifies conscious reactions, and the new arrangements brought about by foresight and effort tend to become organic and natural, and thus to dispense with the supervision of will. Affects become ends, and ends lapse into affects.

What is lost in interest and psychological complexity by the law of habit is more than gained in increased definiteness and reliability. That which is once performed only after a struggle, must be struggled for again : 
but every victory strengthens habit. It is only when a. moral conquest entrenches itself in character, away from the turmoil and uncertainty of the issue gained in particular choice, that it is altogether a conquest. His. virtue is safest who never knows his virtue.

\section{$\S 4$. Motive.}

All the stimuli to voluntary consciousness now discovered may be gathered under a single term, i.e., motive, which shall denote any influence whatever which tends to bring about voluntary action. Motives are seen to fall into two great classes according as they represent pictured objects of pursuit, or the subconscious, organic, habitual, or purely affective, springs of action whose main influence is the coloring they give to consciousness as a whole. The former class of motives are ends, the latter affects. No sharp line can be drawn between them as stimuli; for, as has been seen, they pass constantly into one another. Yet in consciousness the line is both plain and important. As will appear below, it is only ends which are available as distinct lines of direction for volition, in definite cases of choice.

Motives may be classified, accordingly, as follows-the arrows denoting the way of development up and down in experience.

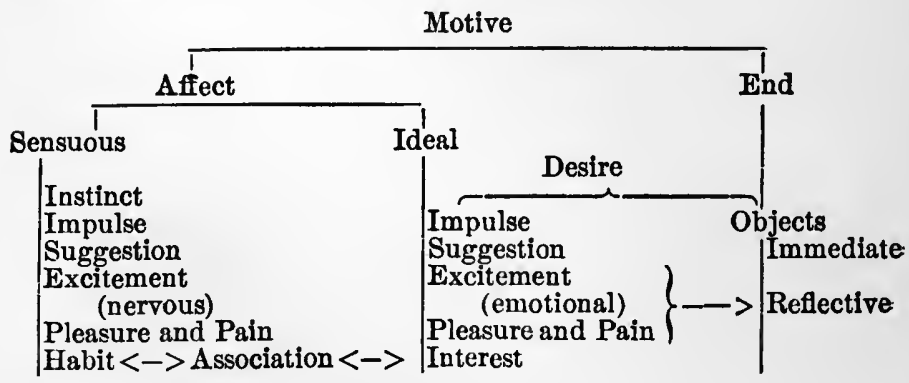


Martinean's classification of "springs of action" under two great divisions, primary and secondary,' corresponds roughly to this dichotomy of affect and end. Differences of detail, however, appear on closer examination. In the table above, the attempt is made to come close home to the psychology of action, and, as far as possible, to give fixed significations to the terms already generally used without exact agreement among authors; and, at the same time, to recognize popular usage. The earlier treatment of ethical feeling prepares us for a distinct ethical spring of action, motive to duty, apart from the coefficient of the right-a contention denied by Martineau, but upheld by Sidgwick. If the recognition of the right is the sole motive to its performance, it is difficult to see why the same individual varies so in his response to it. Spinoza gave us long ago, in his "intellectual love," an affirmative answer to the question of the existence of a more special motive to duty."

On desire, consult : Perez, Él'ducation dès le Berceau, part I. chaps. I and II; Volkmann, Lehrbuch, \$\$ 139-145 ; Kant, Anthropologie, $\$$ 72-84; Sidgwick, Methods of Ethics, bk. I. chap. Iv ; Lipps, Grundthatsachen des Seelenlebens, 6te Absch.; Green, Prolegomena to Ethics, bk. II. chap. II ; James, loc. cit., II. pp. 549-59, and his references, p. 558; Bradley, Mind, 1888, p. 1; Wundt, Phys. Psych., 3d ed., II. p. 463 ff.; Martineau, Types of Ethical Theory, n. bk. I. chaps. V-VII; Beneke, Lehrbuch, chap. v; Sully, Outlines, pp. 574 ff.; Bain, Emotions and Will, part II. chap. vill ; Radestock, Habit and its Importance in Education; Guyau, Education and Heredity; Fortlage, System der Psychologie, \$§ 62-67; Setchenoff, Etudes psychologiques, chap. I.

Further Problems for Study :

Rise, formation, and control of habits;

Habit in edueation;

Relation of habit to character;

Ethical bearings of the doetrine of desire.

1 Types of Ethical Theory, II. pp. 129 ff.

See Pfleiderer, Philosophy of Religion, Div. I. vol. r. pp. 55, 56. 


\section{CHAPTER XV.}

\section{VOLUNTARY MOVEMENT.}

So far the springs of voluntary action have been explored. What do these springs lead to? In other words, what is voluntary action? Confining ourselves as before to muscular movement, we find two great kinds of experience attaching to all movements which we are willing to claim as our personal performances. These we may call respectively feeling of effort and feeling of consent. We are willing to claim any movements of our bodies which we consent to, or which we make an effort to bring about. These two feelings may be considered more closely.

\section{§ 1. Feelings of Effort and Consent.}

What is meant by muscular effort, as a type of experience, is clear when we examine a particular act of voluntary movement : say lifting the arm to a definite height in front of the body. Omitting the elements already found present in reactive or mechanical movement, two great cases of effort present themselves-cases which we may call positive and negative: effort to $d o$, and effort not to do. In positive effort, we strive to bring about movement: let us call this feeling the fiat of will. In negative effort, we strive to put an end to a movement, to control or suppress it: this we may call the neget ${ }^{2}$ of will. For example, I am charged with not moving a

1 The word nolition, used by Preyer, is a happy designation for negative volition. 
paralyzed arm, and I reply: "No, but I tried to!"- this is the fiat. A child is blamed for moving, and he cries: "Yes, but I tried not to !"- this is the neget.

There are certain new factors involved in a fiat of will, factors both psychological and physiological.

Psychological Elements of the Fiat. 1. First, there is a conscious selection of the course to be pursued. I agree with myself, as it were, that my right hand is to be raised, to be raised so high, so high in front, etc. The end of desire is clearly emphasized and cleared of all extraneous uncertainties. There is a feeling of the richness of alternative possibilities, of more or less deliberation upon them, and of satisfaction as to the readiness of all the apparatus, as far as my selecting activity can go.

This feeling of preparation by selection and exclusion, of the adoption of the particular alternative for realization, is altogether new in consciousness. There is nothing like it in simple reactive movements. There, I do not know the real nature either of the stimulus or of the movement till the reaction is an accomplished fact. Here, I know what movement I am to make and why I make it. In short, here is a clear conscious case of end as already found in desire considered as stimulus to will; a sense of adopting, accepting, ratifying, this particular end as my own present desire.

When the muscles have not before been voluntarily used, there is a feeling of separateness, aloofness, from the bodily apparatus; of a futile attempt to select. Let the reader try for the first time to move his ear. We feel in this case that we could, if we could only find the right button to press, the right fulcrum on which to rest the lever. There is a distinct consciousness of search, located in the side of the head.

2. There is, second, a feeling of the waxing importance of this end to me in my consciousness. It persists steadily there, grows large, overshadows every other 
claimant. It is as if my cheeks were being distended by a wind from within-larger and larger, till it is all that I can hold : but still I hold it, and I feel that I alone hold it. No one helps me or hinders.

This feeling of enlargement, of absorption in an idea, is found also in the reactive consciousness. Sometimes an idea emerges uninvited from the background of sensibility, and stalks boldly before the footlights of consciousness, throwing a shadow over all the occupants of the front rows-and holds me against my will. In the present case, however, there is a coloring of feeling flowing forward from the end-feeling (1, above), and backward by anticipation from the fiat-feeling ( 3 , below), which is absent in cases of involuntary enlargement in consciousness.

3. The feeling of fat-Let it be! Let it go! I hold in no longer. The time is come for action and $I$ act. Here the feeling is absolutely peculiar to the voluntary life. It is the kernel of felt self-agency. The outburst of the reactive consciousness is accompanied by a helpless, runaway-horse, feeling: but here the outburst is felt as the urging on of a steed well under rein. This is the consciousness of volition proper.

4. A feeling of control over the muscles : of ability to stop in transitu, to withlold the fiat. The same feeling extends also to the mental flow.

5. A feeling of antagonism to the muscular system. "I tried to" is urged and accepted as sufficient answer to the charge "you did not act." James has called this element of consciousness the "dead lift" of effort, and it is here that effort proper seems to be something added to the volition-feeling. The muscles lie like lifeless wood against the outgoing of one's force. It carries with it consciousness of difficulty, resistance, volition and yet stronger volition, with the felt expenditure already characterized. 
6. There is an intensifying and enlarging of the relational complex of which the end is a part. By acting we know more about the act. The particular reaction gets itself compared with others, throws light on the actor's capacity, precision, strength, and forms a valuable measure for the carrying out of future desires of a similar kind.

7. Finally, we have distinct sensations of movement, if the member move : an agglomerate of touch, temperature, and muscular sensations. In normal circumstances, if there be no actual movement, these sensations are not felt.

Physiological Accompaniments of the Fiat. On the physical side we find, when voluntary reactions are well established, certain significant facts.

1. An enormously increased complexity in the muscnlar apparatus available. This is in most striking contrast to the simplicity and uniformity of reflex and impulsive movements. The latter stimulate particular reactions which are repeated in fixed and comparatively simple muscular arrangements. Voluntary movements, on the contrary, break up, redispose, and reunite the elements of these reactions in numberless ways.

2. There is a direct increase in energy available in the particular muscles toward which volition is directed. Muscles do more work when they are voluntarily worked.'

3. There is greater rapidity, definiteness, and precision of reaction here than in impulsive movements : and this gain is proportionate to the sharpness with which the end intended is pictured. Repetition tends to improve a voluntary reaction in these respects, since it tends to reduce the carrying out of the pictured end to the type of a compound reflex; the volition only serving to start the flow of nervous energy outwards.

1 Mosso; Orchansky, Archiv fur Anat. u. Plıys., 1889, p. 176. 
4. There is a sustained equilibrium of the motor apparatus as a whole, due to education, and no longer a matter of conscious effort. The infant must learn to hold his head up; and that the adult is really actively engaged in holding his head up all the time is seen in the fact that it falls-he "nods"-when he grows drowsy. So the body is in a state of constant muscular tension called by Béclard " "static contraction." A little careful attention to the limbs enables one to detect these conditions of tension, and release them when they are not necessary. One has never learned to rest properly who is not able consciously to throw his muscles "out of gear," so to speak, and sit or lie as heavy as a piece of wood. It is astonishing how much strength is gained by this absolute repose of the muscles. ${ }^{2}$

Psychological Factors in the Neget. There are certain added elements of consciousness involved in an act of negative volition.

1. A sense of strong clash and conflict, between a present reaction now operating, or about to operate, and the end which I desire and will. It is more positive than the mere separation felt in the "dead weight" feeling. In this case I am actively opposed : I do not urge a lazy horse on, but I rein a fiery horse in. "I moved, but I tried not to." This is negative effort proper.

2. When it is a voluntary reaction which is negated,

1 Revue Philosophique, Oct. 1890, p. 401.

2 A book recently written by Annie Payson Call, Poner through Repose, enlarges upon this important fact. The general realization of some means of relieving the "static contraction" of the average American would be a public gain. The writer gains this rest by fancying himself away from all possible interruptions, as lying on shipboard on a smooth sea : it is greatly helped also by consciously imitating the appearances of sleep-breath by slow deep inbalations and quick exha. lations, etc. Every five minutes not actively occupied should be seized upon for such relaxation of the muscles. 
there is a feeling of "calling one's self off," of withholding the nerve-energy necessary to continue the function. This is negative volition. If the function continue, it is involuntary, and I oppose it by "negative effort."

3. In many cases there is a feeling of helplessness and of casting about for means to circumvent and prevent the nervous discharge indirectly. This goes perhaps as far as an appeal to others to hold the offending limb and prevent its reaction.

4. Finally, there are sensations of movement from the muscles and joints in action.

Physiological Accompaniments of the Neget. The physical machinery of negative volition is : 1. The stimulation of the muscles antagonistic to those which realize the reaction negated. The injured party who will not bow to his enemy on the street "leans back for very straightness :" when we determine not to smile, we produce a contrary grimace.

2. Experiments show, also, a direct inhibition of the muscles whose reaction is negated.

Feeling of Consent. The feeling of consent is denied by many to have volitional significance : yet the fact that it always involves an idea or end and indicates an active attitude toward this end-that is, an attitude rather than mere apprehension or belief-controverts this view. I do not consent to the fall of the Niagara River, as I behold it pouring out its strength; but $I$ do consent to my child's going to see it. In the latter case there is a clear reference to my will.

'See Orchansky's extremely interesting experiments, loc. cit. He finds, in opposition to Kries, that the release of the muscle already contracted oceurs before the contracting impulse takes effect in the antagonistic muscle (p. 186). 
There are two general types of consent to muscular movement: 1. A simple readiness to put forth the fiat of volition when it may become necessary, or when I may be driven either to affirm or deny. It often represents. the absence of both fiat and neget, by reason of the close. balance of desire in the two directions. It is closely akin to the feeling of irresoluteness and laissez faire. It responds to the vigorous demands upon me of a stronger personality, or acquiesces in a prospective course which it takes no positive effort to accomplish or in favor of which temptation may be strong.'

2. In other cases, consent is a distinct removal of the neget of will, and as such is a fiat, i.e., "at last I consent." By this I mean that hitherto I have refused my voluntary endorsement, have negated; but now I give my fiat.

We are, accordingly, justified in saying that when consent does not fall clearly under either positive or negative volition, it consists in an attitude of voluntary reserve or indecision which is nevertheless a real condition of will.

Summary on Muscular Effort. Gathering up the elements now seen to be present in effort, we find a distinct consciousness of opposition between what we call self and muscular resistance. Consciousness is unmistakable on this point. In the reactive consciousness, the ego-feeling is present, but it is of an ego involved in the general tendency of the muscular adjustments. In the voluntary, it is an ego which inspects the movement beforehand, selects and approves, or withholds itself and condemns. Whatever the ego be, and whatever we may

${ }^{1}$ A muscular example of this is seen in the peculiar twitching, winking, etc., of the face which some find it difficult to overcome: it does not occur when not thought of, but when actively combated it occurs by tacit consent between the very efforts to control it. 
decide as to the meaning of this consciousness of opposition, it yet exists, and must be given the complete recognition due to such a clear empirical fact.

This distinction between active and passive elements in conseiousness seems to present a formidable problem to the affeetive theory of attention as held by Horwicz and Ribot.' Reflex attention may well be a congeries of passive states: but if voluntary attention is, whence comes the feeling of effort? If, however, the feeling were all and we found no results not ordinarily ascribed to feeling-reactions, then their case might be theoretically made ont. But all that we know and do by volition, and never know or do without it, reinforees the claim of effort as a new agency. The current idealism makes the same mistake and throws away one of its keenest weapons against materialism-strange as the case may seem. The idealists (Green) say: "All knowledge is through consciousness, therefore we can never get outside of consciousness : there are no differences between active and passire states of feeling." "Exactly," replies the materialist (Maudsley "), "your feeling of self is passive like everything else: the unity of mind is the unity of the nervous system, and consciousness is an epiphenomenon."

Muscular Effort and the Attention. The first point mentioned above, as characterizing voluntary movement, was the feeling of preparation; i.e., the relating, selecting, adopting of the end to be realized. Now, as has been shown, this selecting of one of many presentations takes place only in the attention: it is either itself involuntary or itself a fiat. If involuntary, it is a matter of reactive consciousness, in which case the resulting reaction in movement is involuntary also. When a man. acts at random, having no time for deliberation, or perhaps no information to deliberate on-throws a mental penny, so to speak, to guide his choice-his action is not voluntary at all.

1 The Psychology of Attention. Jessen's doctrine is an early and notable statement of the affective theory; loc. cit.

Physiology of Mind, chap. vir. 
In all voluntary movement, therefore, there is an earlier fiat than the will to move, i.e., the fiat of attention to the particular idea of movement." In general, the two forms of volition may be clearly distinguished in consciousness. I may attend as closely as I please to an idea of movement, keep it resolutely before me, and yet not reach a decision to perform it. Yet in the cases in which I do reach such a decision, I do so only by concentrating my attention upon the idea to the exclusion of all others. When I am not able to reach a decision, it seems to be due to a defect in my attention; other ideas share it with the muscular idea. Consequently, it is the degree of preparation, i.e., voluntary attention, which leads to the expansion of a presentation till it so fills consciousness as to overflow in volition.

The entire question as to what volition is, is accordingly thrown back upon an investigation of the exercise of voluntary attention. Voluntary movement is only a particular case of voluntary attention. It is not true, therefore, that all volition is reduced to a play of muscular impulses which issue in a direction representing their resultant; unless it can be shown that the voluntary attention is such an effect of the outburst of a nervous storm.

In this position, the text is in substantial agreement with .James, ${ }^{2}$ except that he uses the expression "muscular effort" to designate the sensational content of muscular volition, which is not consistent with the use of the same term "effort" to designate volition itself in the case of attention. The effort is exactly the same in the two cases: in one case it is exercised about " muscular feelings," in the other, about " intel-

${ }^{1} \mathrm{Höffding}$ is accordingly wrong in saying : "This internal preparation does not admit of more minute description. It is the fundamental element in the consciousness of an intended movement." Outlines, p. 318. On the contrary, we find it to be the consciousness of an earlier act of selective volition.

${ }^{3}$ Loc. cit., II. 561. 
lectual feelings." In one case, the object is a motor intuition, in the other a logical relation: the form, the effort, is the same. Hence it is not true that "muscular effort consists of all those peripheral feelings to which a muscular ' exertion' may give rise" (James, loc. cit., 1I. 562, note). It consists of these, plus voluntary attention (effort), plus the muscular sensations peculiar to voluntary attention. James cites the difficulty of nerving one's self to a cold bath on a winter morning as a case of great roluntary effort and little muscular effort : this is true, for the reason that there are two separate volitions, one to take the bath, the other to make the necessary movements. Both are voluntary effort, but the former is harder because it includes the latter. To a paralytic, the case would be different: the movement would be the great effort.

The fact that the muscles are the channels of discharge of all intense presentations makes it seem that all volition must be directed at first to the muscles. But observation of the infant's first acts of volition shows us a different state of things. The child first pictures a situation and strives to attain it, imitate it, reproduce it, quite unconseious of his museles or of their arrangement. But the coneentration of his attention upon the presentation leads to a muscular discharge more or less appropriate: and by repetition this discharge is adapted to the reaction required. The passage over to the picturing of the muscular movements themselves is clearly marked in his consciousness. When he first begins to learn that he acts by muscles, it throws him into confusion, and his efforts lose the efficacy of their native adaptations. Tell a child to try hard to pronounce a word better, and he mouths his sounds in the direst confusion. But when he is guided simply by the sound, and wills it, with no consciousness of his efforts of speech, his success is better. This simply means that these early reactions, while voluntary, are not voluntary movements: the movements are provided for in the native pathways of discharge; what is voluntary is the interested attention held upon the presentation which starts the discharge.

There are three stages, therefore, in the development of voluntary movement: 1. Voluntary attention to a presentation which, in turn, stimulates a native muscular reaction; 2. Voluntary attention to a presentation of movement, which stimulates the movement presented. This is the state of things in all our endeavors to learn 
new muscular combinations, making them our end; 3 . Voluntary attention to an end for which a muscular reaction is a necessary means. This takes us back to the first state of things again. By the process of learning, (2, above), we have gained new adaptations, and by repetition they have become unconscious means, just as the native reactions ( 1 , above) are. So in writing, for example. That is, we find, the organism gives us so much (1), we improve upon it by effort (2), and, having patented our improvements, so to speak, we hand them back to the organism again (3).

This is seen to be different from the account which develops ideal volition from an early form of muscular volition. Bain makes 2, above, the starting point. Pleasure must be associated with a muscular movement, and the muscular movement pursued for the sake of the pleasure ; this, he says, is the birth of volition. To him the mere idea (1, above), with no associated pleasure or pain, has no motive influence whatever. $\mathrm{He}$ is directly refuted by observations of imitative suggestion in clildhood.

\section{§ 2. Physiology of Voluntary Movement.}

Psycho-physical Conception of Will. Omitting, until later, the consideration of voluntary attention, we have still to inquire into the physical process of voluntary movement; i.e., when I hold the idea of a particular movement up before me and try to perform it, what does my trying add physiologically to the simple reaction involved in the same movement when it is involuntary?

We here reach the extreme form of the question of the validity of a psycho-physical conception of mind. Is the will in any sense a consciousness of a direct forceful interference in the play of nervous energies? Are we conscious, in effort, of a modification, from the side of 
mind, of the strict sequence of nerve-processes from periphery inwards back to periphery outwards? Approaching this question with as broad an outlook at its conditions as possible, we may make certain remarks.

1. The adaptation of muscular reactions to definite ends accomplished by volition is, as regards the results it brings about, the same in kind as the adaptations brought about in the reactive consciousness, when volition is plainly absent. The answer to the question whether in will there is a dynamic spiritual principle must be based upon the interpretation of the selective and adaptive function of consciousness in general.

2. Facts have been cited in abundance to show that when consciousness is present, reflex reactions are broken up, nervous processes are abbreviated, adjustments are effected, which are inconceivable as happening in a mechanical system of actions and reactions. Romanes makes the criterion of consciousness in an organism "non-inherited adaptive or alternative action," and holds that it is "necessary to recognize the element of choice (' uncertainty of adjustive action') even though we give it no influence in the chain of physical events."

3. Yet we have had occasion to defend a strictly mechanical view of the interaction of the nervous system and its environment, as far as the law of conservation of energy is concerned.

There are only two ways to reconcile these positions, provided they are well taken. We may hold with Lotze ${ }^{1}$ that consciousness is dynamic; that it adds to the energy of the system by modifying muscular reactions through volition, at the same time that, by its inhibitions and efforts to control other reactions, it suppresses energy ; and that the additions and subtractions neutralize each

1 Microcosmus, bk. Ir. chap. $\nabla . \S \S 5$ 5. A view for which James has some fondness, loc. cit., II. pp. 576 and 584. 
other in the long-run. This preserves both the dynamic value of consciousness, and the proper balance of the nervous system in relation to its environment.

Or we may hold that consciousness enters as an apparently new principle in the integrating life of the organism-a principle which develops by laws of its own, but which develops apace with nervous integration: that the reason of their concomitance, of their mutual dependence, lies hidden; there are no analogies in nature by which to explain it. ${ }^{1}$ It is clearly a problem of metaphysics; but the psycho-physical facts involved point to some kind of underlying unity which has such a twofold expression. .

As commonly held, this alternative takes a form which appears to present an easy solution to the difficulty. Many hold that while consciousness, will, does not increase or diminish brain energy, yet it directs it. Just as the telegraph operator does not add to or diminish his electric current by turning it into one circuit rather than another, so the spiritual agent guides and facilitates the discharge of particular brain circuits. But the reduction of the physical forces to forms of motion seems to raise an insurmountable barrier to the advocates of such an easy solution. For, while the telegraph operator does not use electricity to open his commutator, still he moves to do it, and the energy of the world which went into his motion is taken away from the amount available for electricity. He might, indeed, first turn his muscular energy into electricity, and with it open the commutator. So the influence that makes the connection in the brain must be itself a form of motion of a particle in the brain, and this means taking so much energy from the ordinary dynamic circuits of the

${ }^{1}$ The morphological processes of life in general present an apparent analogy; but if it be true that life always carries the beginnings of consciousness, then the problem in the two cases is essentially the same. 
brain. The only reply to this objection is the metaphysical postulate of an identity, in which the two kinds of energy are brought under the notion of a common cosmic principle. Such a conception is needed to make the directive and selective function of will, as defended by current spiritualism, available in any scientific sense.

On either of these views, the relation of voluntary to reactive consciousuess is clear. In volition, we are conscious of a form of mental activity sui generis : a form present in all consciousness, but now first explicit. The "selection" of passive, the "attention" of reactive, find their fruition in the "fiat" of volitional, consciousness. This at once confirms, and is supported by, the similar doctrine of apperceptive synthesis found implicit in the earliest distinctions among sensations, but only explicit in conception and thought.

On the first of the two views suggested, no conception of the physical process of volition is possible; since, if altered on occasion by a dynamic reaction of consciousness, the brain-process could not be called constant.

If one embraces the second conception, it becomes his task to conceive the physical process underlying volition in such a way that the increased complexity of its integration will support the adaptations which voluntary acts exhibit, at the same time that the energies of the system are kept inviolate. Such a construction the writer does not care to attempt. More data from physiology must be awaited. Orchansky infers from the fact that the reaction-time for a positive (fiat) and an inhibitory (neget) reaction is the same, that the nerve-courses involved are also the same.

Other current alternative theories of the physiological basis of will do violence to the facts on one side or the other. On one hand, consciousness is held to be an "epiphenomenon," " a

I Archio für Anat. u. Phys., 1889, p. 196 (Phys. Abth.).

Maudsley. 
spark, so to speak, thrown off by the machinery of brain: it illuminates its path, and dies away, having no influence whatever on the machine which produces it. This does direct violence to the distinction between reflex and voluntary action, i.e., to the efficacy of effort. One of the most radical adrocates of the biological theory of mind, Ribot, deserts it at this point. "In relation to the future development of the individual," says he, "it [consciousness] is a factor of the first order; ... it facilitates and guides future reactions." 1

Theory of Innervation. Any theory of a uniform nervous basis of will admits certain points, i.e., an efferent process following upon a central process, this efferent process stimulating a muscular reaction which is reported in turn to comsciousness by an afferent process. A further question arises as to the exact locus in this series of the feeling of effort. Do we feel effort when the energy of muscular stimulation leaves the brain, or when the incoming process from the muscles reports the actual movement? Put technically, are effort-feelings entirely kinasthetic, income-feelings; or do they involve also feelings of innervation, outgo-feelings?

Analogy from the general build of the nervous system, as analyzed above, would lead us to look for an element of consciousness from the outgoing or reacting process no less than from the incoming or receiving process.

An adequate justification of this presumption from a physiological point of view was first attempted by Wundt, to whom the phrase "innervation feelings" is due. Recent writers of note have disputed the point, holding that there is no adequate evidence of any such sensations, apart from the kinæsthetic sensations of movement and the traces they leave behind in memory. Space does not permit an exposition of this complex controversy, nor does the theory of will require it. If we hold a dynamic

1 Diseases of Personality, p. 16.

2 See distinction between "effort" and "resistance" feelings in Senses and Intellect, chap. vir. \$ 3, p. 89. 
theory of consciousness, and that effort is the feeling of this dynamic activity, it does not matter whether the physical result is reported to consciousness before or after the muscular reaction. If we hold that consciousness is merely an epiphenomenon, then a sensation of outgo of nervous energy, like any other sensation, would only represent a condition of the organism. Not only might such a sensation be said, on the one hand, to give evidence of the direct effect of a spiritual impulse of will in liberating central energy; but, on the other hand, it might be said to afford sufficient explanation of effort, and render such a spiritual impulse of will unnecessary.

For this reason, the widest difference of opinion prevails on the subject. In favor of innervation sensations we find Bain, Wundt, Sully, Beaunis, Croom Robertson; against them, Bastian, James, Miinsterberg. The best recent discussions are those of Bastian, 'James, ${ }^{2}$ Wundt, and Beaunis. ' The present writer has found evidence of sensations arising from the condition of the motor centres in the discovery that right-handedness develops in infancy only under conditions of muscular effort. 'This cannot be due purely to physiological differences in brain-sides or arms, ${ }^{\circ}$ for in that case, one hand would be preferred in all movements. Preyer seems to be right in saying that, "muscular sensations [of some kind] are necessary pioneers for voluntary motor impulses." It cannot be due to traces left in memory by greater efficiency, dexterity, etc., by the hand preferred ${ }^{8}$; for at tinis early age (seventh month) no such difference in dexterity, etc., was observed. Besides, greater skill in one hand would cause its use in easy movements as well, especially afte: the right hand had been

1 Brain, x. p. 1, followed by a symposium on the subject.

Loc. cit., Ir. pp. 493-522.

- Phys. Prych., 3d ed., I. pp. 400 ff.

- Les Sensations internes, chap. Xr.

- See Science, XVI, 1890, pp. 247 and 302.

- So explained by Munsterberg in a letter to the writer. Prof. James at tirst favored this view (Science, loc. cit., p. 275), but afterwards gave it up-also in a private letter.

- Mind of the Child (trans.), 1. pp. 254-55.

- James, reference in the last note but one. 
called out predominantly in effort; but this was found not to be the case. The evident explanation is that the child had a vague consciousness of greater motor readiness, outward pressure, high potential, in the centre which stimulates the right. arm.

On voluntary movement, consult: James, loc. cit., chap. XXVI; Stricke, Das Bewusstsein, chap. Ix ; Höffding, Outlines, vIr. A; Wundt, Phys. Psych., 3d ed., II. pp. 487 ff. ; Bertrand, Psychologie l'Effort; (development of) Preyer, Mind of the Child, part II; Schneider, Mensch. Wille, Theil Iv; Sully, Outlines, pp. 591 ff.; Ladd, Elements, part II. chap. x. \$27; Bain, Emotions and Will, part II. chaps. Il-III ; Drbal, Lehrbuch, $\$ \S 135,136$; Perez, First Three Years of Childhood, chap. II ; Carpenter, Ment. Phys., bk. I. chap. IX. 1 ; Morell, Outlines of Psychology, pt. vi. chap. II ; Setchénoff, Etudes Psychologiques, chap. II.

Further Problems for Study: Innervation sensations ; Philosophical significance of effort and resistance. 


\section{CHAPTER XVI.}

\section{VOLITION.}

Purpose. In the last chapter, we found that voluntary movement is only a particular case of voluntary attention. The preparation for movement involves the selection of a particular presentation, and its accomplishment is only a matter of the reiteration of this selection when the proper ideal and motor conditions are present and fill consciousness. For example, I determine at twelve o'clock to dine with a friend at six. I have selected and willed this act: but in the mean time other ideas-knowledge of the hour, present duties, etc.-occupy my consciousness with the intended act. My state of will is then purpose. When six arrives, these presentations foreign to my purpose disappear, the dining act alone persists, fills my attention, and I walk to the house of my friend. My volition at sir repeats my volition at twelve, except that the two involve a somewhat different background of accompanying consciousness. ${ }^{2}$ In both cases, I give myself with all its immediate consequences: in one case, these consequences are apparent only in my mental life; in the other, they shed themselves out through my muscles into the physical world. If I resolve to break into a house, I am a burglar, thongh I be arrested before I move a muscle. Hence, there is only one fiat, one volition, and that is to give my attention to a presentation.

1 "A resolve," says James, "involves all the elements of a motor fiat except the word "noro." Loc. cit., Ir. p. 561. 


\section{§1. Voluntary Attention as Choice.}

Law of Motives. Volition, considered as an act of attention, always involves some measure of division in consciousness-some measure of confusion due to unadjusted claims. The various classes of claims which are to be adjusted in an act have been pointed out. They are the springs of action or motives, any affective tendencies whatever that represent active conditions of consciousness. My whole personality, as has been made clear, is an expressive thing: its expressive side is as real and elementary as its receptive side. Consequently, at every moment the man is expressing himself somehow, and what he is expressing is the outcome of all the elements in him which seek expression.

Farther, the whole of the present possibilities of the man are summed $u p$ in these tendencies outward: they represent his entire self at the moment that he acts, i.e., his make-up as the present conditions of his environment are suited to call it out. Given conditions which favor the expression of a number of his motives at once, and they all clamor for exclusive recognition. For example; a brakeman's hand is freezing to the iron: intense pain, a physical spring of action, prompts him to desert his brake. But he quickly calculates the chances of collision, or an open bridge : an intellectual motive urging him to remain faithfully at his post. And with this last there come the picturing of wounded passengers, the cries of those in danger: a new emotional motive, which brings with it a warm flood of sympathy, leading to a quick and easy decision on the side of duty. The decision is the man's decision; it expresses the nature of this man and no other; and it is the outgoing of his nature in a line which the particular circumstances opened to him. Accordingly, we may say, first, that all 
volition results from a more or less complex aggregation of motives; and, second, that this aggregation of motives exhausts the possible alternatives of present action.

The first position is clear from the analysis of the affective basis of volition above, in which the different stimuli to volition were pointed out. It is impossible that any one of these should act alone, for a min is never free from his body, on one side, or his higher ideals, on another side, or his emotional tone, on a third. They are all present always in normal life.

The second position shows us that any doctrine according to which a man can transcend his motives, hold aloof from them, despise and reject them, simply asks us to chase a fije-fly. If you remove a man's motives you remove the man; for what is the man but body and mind? The whole content of volition disappears. 'To will at all, a something must be willed, but this something is a pictured something, bearing some relation to myself. The reason $I$ will it is because it moves me-is my motive. Let me picture never so strongly the fabulous - the utterly uninteresting and indifferent-and will in reference to it is impossible. I can never make new motives, or will a thing that does not for some reason find a responsive echo in my breast.

Nature of Motives. It is also plain that a motize is nothing in itself. It is only a name for a partial expression of the nature of an agent. Consequently, motives can in no sense be considered as forces which expend their energies upon the will, or which fight each other. These conceptions of current psychology are nothing short of myths-myths which have "darkened counsel without wisdom" long enough. Apart from the motives, there is no will to fight against, and as to struggling with each other-that would mean either that each of the motives had a will of its own, or that there were no common life whose full realization is the best satisfaction of them all. Here is a developing principle-call it what we may - whose different life-furthering adaptations represent a hierarchy of worths. One worth is chosen. If it be the best, the others are also furthered 
with it by their very denial : if it be lower than the best, it suffers with the others through its gratification; both because, as elements of a common life, all are involved in the gratification of each. How, then, can they be conceived as separate entities contending in a theatre which is cold stone to all of them? Rather, they are all vital elements in the functional synthesis of a living consciousness.

Affects as Motives. Among motives, two great classes have been distinguished, affects and ends. The former are immediate influences upon the will, unpictured, unreckoned, unavoidable. The latter are reflective motives, pictured, estimated, subject to conscious selection or rejection. Now it is plain that these two classes of motives stand on very different planes in the mental life, as regards their volitional worth. If all volition is in view of an end, then it is only by strengthening the influence of particular ends that affects enter. If I grow greatly excited, for example, over a particular choice, my excitement colors my choice only in so far as it presses home upon me one alternative of my choice. My physical health alters my opinions and reactions, not by supplying me a new end, but by brightening a consideration here, dulling another there, rendering the attention sluggish, and so limiting the range of my consideration, or stimulating it greatly and so pitching the entire intellectual play at a higher key. What actual volition is concerned with, therefore, is ends and ends only.

The clearest example as well as the plainest demonstration of the influence of such subconscious motives upon choice is given by recent results in hypnotism. A Auggestion given to a patient, with instructions to carry it out at a certain hour after being restored to his normal condition, is car-

${ }^{1}$ Binet and Féré, Animal Magnetism, pp. 207 f. 
ried out when the time arrives. The subject acts voluntarily, pursues a present end, but declares that he can give no reason whitever for his conduct, as no trace exists in his memory of what occurred during the sleep. The stimulus to the will must have remained somewhere in him, though not in his consciousness. Of course the new hypothesis of a subsidiary or split-off consciousness suggests itself here.

Volitional Apperception. How, then, does an end pass into a volition; how does it get the fiat which makes it an act? Careful questioning of consciousness leads us to see that the picturing of ends is in no respect different from the picturing of anything else. It is an ordinary act of apperception, by which new elements of conscious content are taken up in an integration with the old-established complex of presentation. The new end gets in only as far as it is adjusted and harmonized with old ends: tho old ends themselves, a single integrated group, take on a new complexion from the new element of experience thus absorbed. The attention moves throughout the series of elements, grasping, relating, retaining, selecting, and when the integration it effects swells and fills consciousness-that is the fiat. Just as soon as the elements of the end-complex cease to act as partial influences, causing the movements of attention by their own vividness, and the attention gets its hold upon its integrated content as a grand related situation, the fiat goes forth.

For example, I have been accustomed, after careful thought, to pursue a given line of business policy. It is the outcome of all my thinking, feeling, and past action-an integration, a motor situation, which exhausts my motives and represents my present volitional attitude. A friend gives me new information; it gets an entrance by its own intrinsic hold upon my attention; it becomes an element in the situation; every other element gets a new adjustment; and when I make up my mind again, get control of the situation through relative 
stability in the apperceptive outcome-then I am at once in action-my fiat is given.

Now no one end has brought about this result. I do not adopt one and utterly deny others. I adopt the situation in which all have entered and to which they have given each its own significance. It is true that the exigencies of conduct narrow me down to a very small number of expressions. I must either go to the opera or stay away. But neither alternative represents my true mind. I decide to go, since; to stay away, if; and whichever I do, it is with the clear consciousness that I am not realizing my ideal volitional situation in the premises. Instead of indulging one of my ends, I am acting on a compromise, which really satisfies none.

Volitional apperception, therefore, differs from general apperception only in its explicit motor reference. This reference, as has been seen, is present in all apperception; no state of consciousness lacks it. But when I have action in view, the moving quality of the elements of my synthesis is more felt. Generally, my decision is simply consent-the passage of "the adopting act." I consent to a thing when I give it my sanction. This is volition; but not as full a volition as the volition of conduct. When I know that my own fate is involved, that it is I who must act, there is a fulness of emotional warmth and reality that gives new affective coloring to the ends involved, and perhaps radically alters the outcome.

It is readily seen that a new class of emotions is involved in this latter case, the self-emotions, i.e., pride, ambition, shame, etc. As soon as the notion of self gets involved in the controversy, it is impossible to escape their influence entirely. They enter as additional motives, generally affects, but possibly distinct pictured ends of desire. For this reason, it is difficult to make our judgments of ourselves as severe and exacting as the demands we make of others. 
Controlling Motive. The controlling motive, consequently, is the motive which wins the fiat. But it is very difficult to find anything that it controls. It does not exist at all after the fiat, for the outcome of the fiat is a new end in which all the motives have entered. So it does not control conduct, which is the expression of the fiat. For the same reason, it does not control the volition itself. Every one of the motives is controlling in the same sense, i.e., of entering essentially in the result. The only advantage it has over other motives is that it becomes the final channel of expression ${ }^{1}$ in conduct; an advantage denied to them. In this sense it controls the other motives, but only in this sense.

Deliberation. The state of division, balance, and indecision described, is ordinarily called deliberation. Its nature is now sufficiently clear. Its duration depends npon the complexity of the considerations which arise, the evenness of their motive influence, and the absence of pressing urgency of choice. Individuals vary greatly in the thoroughness of their deliberative processes. As a rule, deliberate, slow decisions are safest, though, as has been seen, it is possible that an unexpected flash of conceptual feeling may carry the day in favor of an unseen aspect of truth. An important additional motive in deliberation is the state of mind called caution, arising from a sense of the danger of hasty decision.'

1 Either real or intended expression : it is well pointed out by Bain (Emotions and Will, p. 399) that the act is complete and the motive controlling (in his view because of pleasure and pain associations) when in fatigue or disease there is no energy to carry it out. Wundt's explanation of the fact is nearer that of the text; cf. Ribot, German Psychology of To-day, pp. 202, 203.

Bain makes caution, of course, purely a pleasure-pain motive, and the entire ground of the voluntary delay involved in deliberation; loc. cit., p. $408 \mathrm{f}$. This means that instead of the popular "Be sure Jou're right, then go ahead," our real state of mind is "Be sure you're 
Choice. Choice is the fiat itself-the adopting actas it terminates upon an end. It is volition considered. not as the general form of will, whatever content it may be exercised upon, but a particular volition upon one of alternative pictured ends. A choice is always a definite particular choice. And it includes, as a phenomenon in consciousness, the feeling of the continuance of the partial ends which enter in deliberation. It does not. quench one desire to resolve to satisfy another. And the intellectual act of apperception, whereby the course chosen is constituted, may find itself in need of constant. reiteration to maintain itself. We need to be constantly reminded of the reasons of our faith in order not to lose. it. The greatest moral victories may be subsequently lost through the stolen march of a desire or impulse once successfully subdued. Choice, therefore, is the feeling. of the settlement of a question which is still a possible. question. It is a volitional declaration with a felt interrogation. As soon as our decisions pass out of the range of reconsideration, they are not properly choices. any longer; they become, then, elements in character.

Potential and Final Choice. In regard to the performance of a course of conduct, two stages or aspects of choice may be distinguished, potential and final choice. By potential choice is meant a man's decision as far as it results from his own character, disposition, personal preferences, etc. Potential choice covers the whole. range of affective motives, the dumb unpictured influences which get in their work silently. It includes. also the ends which one's own character, memory, knowledge supply : in short, it represents the decision I reach when "left to myself." It is potential choice that we feel sure about in the case of our friends: it is more

safe, then go abead." He terminates deliberation by the felt danger of indecision. 
approximately a constant thing from day to day. It represents the great currents of our lives, the habitual lines of activity, opinion, and interest, of which more remains to be said below.

Final choice, on the contrary, is actual choice, active choice, acting choice. It is the full outcome of deliberation from whatever sources considerations may come. It is the adjustment, the compromise, as it was called above, of all the actual circumstances of the case. It is choice as a spectator looks at it and asks, what did he do? Not what did he personally most wish, or did his action satisfy his ideal situation? It is, further, in the later stages of deliberation that potential choice suffers the revision which makes volition actual. It is brought about by the more unessential, the less interesting considerations. Many a fond wish is murdered by the present demands of cruel circumstance. It is also here, in the more or less open interval between potential and actual choice, that the estimable qualities of openmindedness and ingenuousness appear. The open-minded man is receptive to new suggestions, arguments, and emotional appeals. His habits of action have not become so petrified about him as to block up the cliannels of new volitional reaction. Others "are not so, but are like the house which is founded upon a rock." Nothing but an earthquake can shake the man whose potential equates with his actual choice regularly.

Feeling of Alternatives. The feeling of open alternatives which is said to characterize choice rests, when an act of rolition is closely scrutinized, in one of two places : either before the volition, during deliberation, or after volition. Before volition, the possible alternatives are actually present as candidates for the position of controlling motive. We know that one of them and only one will be the final channel of expression. Any one is eligible 
for this. They are really alternatives also in the sense that the outcome is not yet foreseen : consciousness has not yet reached the stage at which there is any outcome at all. But these two considerations exhaust the meaning of felt alternatives before volition. This feeling is further complicated with that of obligation.

After volition, as already said, the motives persist. The circumstances of deliberation throng back upon us: especially after a hard long-fought decision do we live by retrospection in the past. But further than this, we feel that another revision is possible: that new light may come to us and our decision may be reversed. Here again, therefore, are two senses in which alternatives are felt; one, the persistence of the conditions, of a choice already made, the Nachklang of our effort, the drifting smoke of the battle-field : the other, the gathering again of the conditions of choice, the preparation of a new choice. This latter, therefore, is identical with the similar feeling before volition. Accordingly, the feeling of alternatives is always a sense of contemporaneous motives or of reminiscences of such.

As to volition itself, however, it is accompanied by no feeling of alternatives. On the contrary, it is felt as a peculiarly exclusive, definite, intolerant thing. It terminates alternatives, and fills consciousness with a single apperceived presentation. As Ribot phrases it, voluntary attention is a state of monoïdeism. If $I$ attend to two things at once, it is because I will both things ; together they give the end. The end itself is one and undivided. This cessation of deliberation is accompanied by an emotional coloring of relief which is highly pleasurable; and it is in sharp contrast to the unpleasant tone of conflict which characterizes indecision.

Moral Choice. Moral choice involves the moral impulse as a motive principle. In decisions in which moral 
feelings are not involved, this principle is practically absent. As soon, however, as the cocfticient of the right in conduct is, or is likely to be, disregarded, a new coloring is given to all the phases of the act of volition. In addition to the consideration of expediency which is the unwritten law of choices morally indifferent, the consideration of right enters through the ethical feelings. Each pictured end has its value as relatively fit or unfit for construction in an ideal of conduct.

There are two peculiarities about the moral motive, however, when considered as entering among the factors of deliberation. First, it is not itself a pictured end alternative to other ends. We have found that the moral ideal is not presentable. It is rather realized in the relative adjustment of other ends to one another. Consequently, the moral motive is not realized by withdrawal from the ordinary conditions of action, or by its own abstract pursuit; it does not present for itself a distinct channel of expression. It enters to dignify and justify one of the ordinary series of alternatives, as of more worth in a scale of moral values.

Second, the moral motive, as said in an earlier connection, carries with it the felt authority of a categorical imperative. I may decide on the expediency of a course and then disregard it, with no blame, no remorse: but when I decide on its rightness, this very decision is a recognition of an authority beyond which there is no appeal.

Choice and Habit. In the sphere of volition, as elsewhere, the law of habit has striking applications. Ends tend by repetition to coalesce with one another. Complex series of volitions become so closely integrated, that a starting fiat is all that is necessary to bring about a series of well-adjusted motor reactions. Here, again, two great views of habituation open before us. First, 
the voluntary shifting of attention, the effort to select, arrange, accomplish, becomes unnecessary by the law that association takes over the work of intelligence. Thus the surface of consciousness is made more calm from moment to moment, and the attention is left free for new fields of exploration. Such a combination of elements in a single voluntary movement we may call an act. Thus, opening a book and turning to the place desired is an act: but it represents innumerable efforts, failures, and partial successes extending over years of child-life. An act is what was called in an earlier connection a " motor intuition."

Second, these acts get segregated in like manner; lose their individuality in what are called dispositions. Our acts grow more and more alike: our day's devices become routine: our satisfactions vary with our education, and fall back under the lead of impulse. Nothing, in short, in which our agency is involved, escapes the solidifying, unifying effects of habit."

The result is that ends get back to the status of affects, and our voluntary life becomes more limited in the range of clear consciousness. Even the power to rebel against a habit is itself a matter of habit. A habit is hopelessly fixed when there is no disposition to break it up.

Hence the extreme importance, on the part of teachers, of a clear understanding of the laws of volition in its early rise and progress. Variety should be everywhere provided in the tasks for children. Choices which involve self-denial should be dwelt upon, illustrated, and encouraged. No pains should be spared to give the child an intelligent view of the claims of others upon him, in order that the habits which he does form may be beneficent and moral.

1 Senses and Intellect, chap. vir. \$ 6.

${ }^{2}$ Cf. Lotze, Microcosmus, r. p. 255. 
Intellectual Effort: its Forms. Effort to accomplish an intellectual fask is characterized by the marks already found attaching to muscular effort. Indeed the latter is but a particular case of the former. The effort to keep up a train of thought, to suppress an emotion, to bring order and coherence into the mental flow, has the same feelings of fiat, dead-lift, resistance, already found in the earlier case. If we can manage to keep the attention well fixed upon the object of desire, the battle is wonit swells and fills consciousness, and wins volition.

Special forms that more intellectual effort takes are resolution, 'determination, perseverance, doggedness : all the manifestations of so-called strength of will. They all express the more or less habitual exercise of attention as it gains control and comes to characterize the individual. They refer more especially to potential choice, as reflecting character.

\section{§ 2. Character.}

The conception of character, apart from the metaphysics of it, properly attaches to the active side of personality. It means the essential part of a man, that which is most himself, but it is interpreted, like everything else, in its expression. Action is the only and the adequate expression of a man. So character means the present agent, the possible actor. The notion also includes the idea of permanence. Character is that expression of a man which is most constant, habitual, and, in consequence, most unconscious, unpremeditated, genuine.

While the most permanent expression of personality, nevertheless character is not a stationary thing. It is a progressive, developing thing. Especially in early life, the change and development of character are super-

'See Buin, Emotions and Will, r. p. 417. 
ficially evident and present the only adequate statement of the problem of education. As has already been seen, the growth of mental function as a whole waits, in early life, upon the growth of the physical organism; in later life, it becomes more independent, developing under the law of volition; but in both cases it is still, with the physical organism, subject to influences from the conditions which envisage the personality as a whole.

We may speak of the "innate gift of nature" as a man's endowment, that which he starts with, received by inheritance. It includes all his potencies for development as far as they can be conceived apart from the external conditions in which alone they can be developed. On the other hand, the sum of these external conditions from birth upward, considered as influencing character, we may call environment.

The question as to the nature of present character, is accordingly this: what is the law of the development of a man's endowment in relation to his environment? Two great principles already arrived at find further application here, i.e., the principles of adaptation and habituation.

Development of Character through Choice. It is by choice, that these principles get their application. Choice plays the part in the development of character that nervous reactions play in the development of the sentient organism. Nervous reactions were found to be to a degree selective and adaptive; and further, it appeared that such adaptations become fixed in structure: by the principle of habit. So choice is selective and adaptive, and its reactions create tendencies toward those habitual performances which are the outcome of character.

It is in final choice that the reaction of endowment. upon new environing conditions becomes evident. A 
man's potential choice represents that which is already in him. Any modification of potential choice is due to influences from without, to enviromment. The consequent reaction tends to identify the man with the new consideration before foreign to him. He has taken it up in his deliberation, given it a place in the list of motives which appeal to him, and thus disclosed a desire, whim, preference, now more important to him because he once has harbored it. Character, accordingly, as an expressive thing, has thus taken a step in its development through the influence of environment.

The potential choice of a man at any time, therefore, represents all the final choices of his past life. Each link in the chain of volitions, from the present back to his first exercise of choice, has involved these elements. The very first act of choice of a human being is already expressive of the accommodation of himself to his circumstances. Indeed, it is through the stress of circumstances, through the necessity imposed by muscular resistances, violent pains, and crying appetites, that volition in the first place takes its rise.

Accordingly, it is easy to see that environment enters. in the development of character in three ways. First, the way we have already seen : it presents new ends for choice. Second, it becomes a conscious influence orer our prospective choices. We decide our questions subject to future light, circumstance, fortune. The character thus grows pliable, the will cautious, action hypothetical. This result of environment is a more complex and refined application of the law of habituation. Where uniformity of experience prevails, action grows habitual. Where lack of uniformity prevails, distrust and caution grow habitual. The latter is more unusual, since uniformity is more easily seen and accommodated to: but it is equally real-the tendency of reflective thought upon the relative values of experiences, to make men 
sceptical in their opinions and unenthusiastic in their deportment. It simply means that indecision, which is the enemy of habit, paralyzes volition; for habit makes volition spontaneous and impulsive.

Third, the principal influence of environment is undoubtedly before and during the early rise of volition. In very early childhood, authority is the controlling influence in moulding actual choice, and thus in fixing character. So important is this that some writers ${ }^{1}$ find in the "word of command" the foundation of all subsequent authority, moral as well as legal. However this may be, the observation of children shows to what a remarkable extent the authoritative suggestion of a parent sets the inclinations and forms the habits of his child. Even in the matter of physical appetites, likes and dislikes may be to a large extent controlled. ${ }^{2}$ Imitation and suggestion start reactions which become habitual. The unconscious lesson of a bad example learned by a child from his father is one of nature's most impressive pieces of moral instruction. Moral contagion of character is as direct and unconscious as physical contagion of disease. Further, early social conditions, family, school, and play associates, create a milieu which makes endowment practically helpless as to the methods of its expression during the early years of life. Educationally, the tremendous influence of environment is the more apparent since it is just at this period that the child begins to reach those conceptions which serve as point of departure for moral feeling.

The relative strength of heredity and environment is thus a matter of varying adjustment. In many cases the virlie growth of inherited tendency may outlive and defy the most

1 Bain.

2 The diet of a child from one to two years of age may be made the basis of permanent appetites by judicious suggestions of relish and enjoyment. 
careful or careless training. Galton shows this in his interesting inquiry touching the development of twins.' It certainly is not to be expected that the environment of a single lifetime will undo the acquisitions of generations in environment equally forceful. But generally, the effects of heredity are not so uniform in fixing positive traits of character. A child inherits not only from his immediate parents, but from theirs, and Galton's "law of reversion to type" would tend to neutralize those prominent traits which survive. Consequently an important part of character, it is safe to say, remains to find its form of development in the conditions environing the individual life. The development of character may be represented by a line ( $x$, endowment) broken by the recurrent intersection of other lines ( $y, y^{\prime}$, etc., environment), the angles thus formed being points of choice $\left(a, a^{\prime}\right.$, etc., potential, and $b, b^{\prime}$, etc., final), as follows:

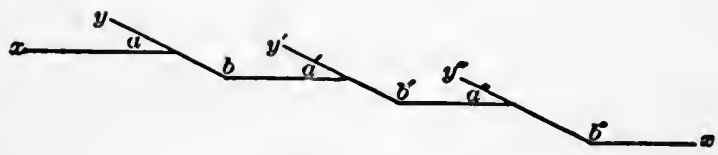

\section{§ 3. Intmiation op Motives by Attention.}

Coming closer to the actual method of voluntary attention, we seem to find a wide range of apparent exceptions to the law of motives as now stated. The attention, we know, intensifies a mental state. It is possible simply by dwelling upon a consideration to increase its importance to us, to give it preponderating influence in our deliberation, and, finally, to convince ourselves of its supreme desirableness. It looks, if not like the initiation of new motives by the attention, at least like the initiation of new intensity in old motives. This effect is further exaggerated by the fading out of other motives in consequence of the withdrawal of the attention from

1 Inquiries into Humun Faculty, pp. $216 \mathrm{ff}$. 
them in favor of the "star actor." The important question is: Is this exercise of the attention itself unmotived-independent of the conditions of endowment and environment already pointed out? This question must be answered in the negative, for several reasons.

1. Such a result often follows upon the involuntary exercise of the attention. By a sudden stimulus from without, the attention is shifted, leaves the chain of deliberation, dwells upon an alternative before subordinate, and so changes the throw of volition. A burglar greedy of gain contemplates a robbery, but a harmless noise starts associations which suggest danger, and he deserts his enterprise. Any incident which arouses the attention from its line of easiest passage, and gets it concentrated upon a different train, is apt to modify choice. So lawyers aim to divert the attention of jurymen from the claims of mercy by exhibiting bloody weapons, dwelling upon terrible incidents, and thus getting the attention under the lead of strong emotion. In these cases there is clearly no factor apart from the environment and the elements of character which respond to it.

2. It seems possible to divide all cases of such apparent initiation of motive intensity into two classes; one, the cases of involuntary attention mentioned, and the other, cases of deliberation. If I have no intention at all in the matter, no trace of preference for the motive whose intensity is strengthened, then it is clearly involuntary-a matter of the reactive consciousness. But as soon as any such preference comes in-any physical, mental, or emotional motive for wishing to intensify this particular alternative-then my choice is already made, and I am fooling myself in thinking that I am reaching an unbiassed decision. Most of the instances are of this latter kind. They are the becoming conscious of the 
great class of volitional stimuli already described as affects. Habit, for example, becomes conscious in its influence on volition; vague physical and emotional states which are never distinguished from the fundamental tone of our personality reveal themselves thus, as elements of it.

Hence we may conclude that this phenomenon is only a phase of the general mystery of attention. By attention, deliberation takes place, and choice is the outcome of this deliberation. When we are absolutely outside the range of deliberation, instead of finding ourselves in the presence of altogether unconditioned activity, we only revert back to activity of the reflex type.

\section{\$4. Freedou of the WiLL.}

In the light of the foregoing, the problem of the freedom of the will takes at least an intelligible form of statement. Freedom of the man is perhaps a better way of stating it. Yet the term freedom suggests a comparison with the conditions of physical causation which is essentially misleading. It would be well if this discussion were thrown into terms of psychological meaning independent of popular usage altogether. The statement of the following alternative views may suffice to bring out the real point at issue in the free-will controversy.

I. Indeterminism. On this view of volition, choice is absolutely unconditioned. The will, or the agent through the will, asserts itself as it sees fit: it is in no way conditioned either upon motives, brain activities, or external circumstance. Pure indeterminism is also called "accidentalism." In opposition to such a view of volition it may be said:

1. It is altogether unpsychological. The most thorough search of consciousuess discovers no such cases of 
absolutely unmotived choice. 2. It leads logically to one of two alternatives: either the will has no relation whatever to its social and physical environment, in which case it can have in turn no influence of any kind upon it; or it moves by chance, whim, caprice, which if true would violate the uniformity and stability of character. 3 . It is altogether unnecessary for the purpose for which it is usually urged, i.e., in the interest of moral responsibility and obligation; for an unrelated will would be responsible to no authority, and a will that moved by chance would know no law. Indeterminism is claimed chiefly by the unreflecting, who fail to see that in holding volition to be motiveless, they cut off the agent himself from all voluntary expression.

II. External Determinism: the view of all those who by any method bring volition within the chain of natural cause and effect; all who hold that there is no activity in the voluntary or relational consciousness not reducible to motive forces. On this view, that is, motives are forces in reference to one another, effects in reference to the brain in which they have their causal support ; volition is the consciousness of the outcome of a conflict of forces. It is part of the "epiphenomenon" theory of consciousness already explained. This theory in turn evokes several criticisms.

1. The theory begs the difficulty of passing from the external to the internal: from a brain process to consciousness. It forgets that this gulf has not been crossed. To assume a uniform psycho-physical connection is a very different thing from assuming that consciousness is an epiphenomenon. If determinism ever be established at all, it will be a determinism which reduces volition to other states of consciousness, not one that presumes to blot out consciousness altogether.

2. After we get in consciousness, we have no right to 
apply the law of physical causation to motives. It is a most warton assumption from every point of view, except that of physical analogy. Motives persistently elude the application of the symbolism of natural causation. Where in the play of motives is the law of resultant? Statistics showing uniformity of marriages, crimes, etc., in a community, simply prove that men have a common nature, and are appealed to by common motives; and that variations of choice positive and negative equate with each other. The same is true of the number of drowning accidents on the sea-coast, and it would be just as logical to claim that all who were drowned were pushed into the water and held under as to claim that uniformity in the aggregate indicates cause and effect in individual choice.

3. Physical causation presents us no analogy to the selecting, intensifying, abbreviating, and synthesizing activity of attention. As far as the analysis of physiological function has gone, reflex action is its purest type; yet even in the cerebral processes which underlie volition, directive modifications of the reflex have to be presupposed. Even though the law of conservation sweep through the brain, as we hold it does; yet it is only when selective consciousness is present, and presumably because it is present, that the resulting reactions are what they are. In order to prove the position, apperception would have to be reduced to association, and association made a function of cerebral dynamics only.

4. As a matter of fact, we know no external influence which can compel the will. When we do influence another it is by previous knowledge of his inner character-the mental habits spoken of ; but that, at its best, is by no means a certain device. It is true that if there were no other consideration against motive determinism, this fact might be considered due to the complexity of 
the forces involved: but in the fact of the conscious synthesis of choice, it seems to have a readier explanation.

III. Immanent Determinism. This doctrine holds. that there is in man a principle of realization - the realization both of himself and of an universal consciousness through him. In volition this principle attains advancement. The innermost nature of a man is, therefore, necessarily expressed in every act of choice. It is a free expression of what the man is, and, consequently, of all that he represents as part of the world : but it, at the same time, unconsciously realizes a broader development. in which all individuals are factors.

As far as this theory is psychological, it is tenable. Whatever is immanent must be included in the nature of that in which it is immanent: so volition is, after all, - for psychology, simply the expression of the nature of the man himself.

All metaphysical construction upon this basis, however, is: foreign to psychology. The question then is : Is the position well taken that choice is of such a character as to afford a basis for an immanental view? This is true only on condition that we find some interpretation of choice by which volition is a. conditioned but not a caused thing. As soon as we make it a caused thing, then that which is the immanent cause is no. longer fully identical with the will, i.e., is no longer fully immanent, and we go back to external determinism.

IV. Freedom as Self-Expression. Our view is now narrowed down to very strait limits. The consideration of motives has led to several determinations: 1.

- Choice is never motiveless. 2. The end chosen is. always a synthesis of all present motives, and is adequately expressed by no one of them. 3. This synthesis is an activity sui generis: it finds no analogy in the composition of physical forces.

These positions find their only explanation in the supposition that the existence back of choice includes. 
in its own nature both the motives and the volition.' The motives do not grow into volition, nor does the rolition stand apart from the motives. The motives are partial expressions, the volition is a total expression of the same existence. How the motives pass into or stimulate volition is the law of mental development, a law which has no analogy in external nature. The relation of this law to brain-development is again a higher exhibition of that psycho-physical connection which has been assumed; a connection which is real, but which yet does not prejudice the laws of development on one side or the other. As has been said, this seems to point to some underlying unity in which the antithesis between mechanism and volition is resolved.

Freedom, therefore, is a fact, if by it we mean the expression of one's self as conditioned by past choices and present environment. It is not a fact, in any sense which denies that volition is thus conditioned, first, upon the actual content of consciousness as it swings down the tide of the personal life and presses outward for motor expression; and second, apon the environing circumstances which draw the motor consciousness out. Free choice is a synthesis, the outcome of which is, in every case, conditioned upon its elements, but, in no case, caused by them. A logical inference is conditioned upon its premises, but it is not caused by them. Both inference and choice express the nature of the conscious principle and the unique method of its life.

The much-debated question whether a man could have chosen differently in the same circumstances, is seen to be quite irrelevant; for the reason that the circumstances can never be twice the same. Yet it is plain that to say that the apperceptive process of choice might issue differently is to

1 Spinoza says: "WIIl, considered with regard to mind and body together, [is] notbing else than the being of the man himself." Euh., 11I. 8 Schol. 
suppose some change in its content: some change which brings out another phase of the man's character for expression. But as has been seen, such changes may be so slight, so superficial, and yet may work such results in volitionthat it is no wonder we overlook their presence, and take the case for one of possible mental initiation.

Care should be taken to distinguish between the question of freedom and that of the relation between body and mind. If consciousness is an epiphenomenon, then the external determinist has his way; but if consciousness be a synthetic thing, still the determinist is not necessarily excluded. For a spiritual synthetic reaction may be a link in a series of such reactions causally related to one another. Further, if consciousness do not interfere in any way in the play of brain energies, yet the will might be conceived as an agency which does not consent, which protests against its physical powerlessness, as does the paralytic. Much confusion is current from the failure to keep these two questions distinct. $^{1}$

Feeling of Freedom. The feeling of freedom seems to be made up of two other feelings about equally, i.e., the feeling of alternatives and the feeling of agency or power. The latter is rather a felt reminiscence than a state of original sensibility. It rests largely upon memory of past stimulations or inhibitions of the movements now alternative to one another. Preyer ${ }^{2}$ holds that there is true will only when there is positive inhibitory power over the movement in question in each case. This is clearly not the case in imitative volition, when the movement is attempted for the first time: but yet in these cases past volitions of other movements are sufficient to give the memory of power. It is probable that this feeling of power or agency gets rapidly generalized away

1 For example, Höffding, Outlines, vIr. B. 5, 6.

${ }^{2}$ Mind of the Child, trans., I. pp. 192-95. 
from muscular movements in particular, to alternative ends to which muscular reactions are only means. The feeling of alternatives, as has been seen, also goes before volition, or is also due to reminiscence. Hence the feeling of freedom is subject to the criticism already urged against the sense of alternatives: it depends upon the division in consciousness which $I$ feel it is for myself, my own apperceptive activity, to solve in the future.' At the moment of volition there is no feeling of freedom. Rather, when the fiat goes forth, there is a sense of irrevocableness, of once-for-all conclusiveness; a feeling of having thrown one's self over a moral precipice.

Feeling of Responsibility. As soon as an act has taken place, a new phase of feeling arises, that of responsibility. It arises only when the stimuli to will have been stamped with the seal of one's private ownership. I do not feel responsible for my desires, impulses, emotions, except as far as I have ratified them at some time by my choice. Responsibility is a feeling of a past explicit choice, just as freedom is the feeling of the possibility of such a future choice. As attaching to all final choice, this feeling is called natural responsibility. It is only the sense of ownership in the deed and its consequences. When the motive conditions include a command imposed by an external authority, it becomes legal responsibility; when the imperative of duty is a felt condition in the decision, it is moral responsibility. The feeling of moral responsibility for wrong-doing passes quickly into remorse.

1 Jessen notes this future reference of the feeling, Prychologie, p. 363. Ulrici says. " Freedom of will is only freedom of choice, for all we feel free to do is to choose which of the present impulses we will follow." Gott und der Mlensch, 2te Auf., Ir. 


\section{$\S 5$. Effects of Volition.}

Expressive Effects. The immediate effects of voluntary attention have already been briefly mentioned. Physiologically, we find certain sensations of concentration in the head, principally at the sense-organ through which the stimulus is received. The skin of the head is drawn forward and knotted on the forehead, in visual attention. Experiments show an increase in the bloodsupply in the organ attended to. In attention to a picture of imagination, or in attentive thought, the eyes roll upwards and around, and there is a feeling of exploration or searching in the back of the skull. ${ }^{1}$ In strong effort, moreover, there is a setting of the epiglottis and a compression of the jaws. All these indications are additional to the explosive or inhibitive effect to which the effort itself is aimed, and which it in so far accomplishes.

These expressive changes are rather the accompaniments than the effects of attention. They bear much the same relation to volition that emotional expression does

${ }^{1}$ I can find no ground whatever for Ferrier's assertion that no presentation can be voluntarily attended to that cannot be visually pictured. It is easy to fixate the eyes on a point and at the same time follow a tune through mentally, or to think of a single note without the " indirect picturing of the source, instrument, circumstances" which Ferrier thinks necessary (Functions of the Brain, 2d ed., p. 464). Darwin is much truer in saying (Expr. of Emotion, 2d ed., p. 239): “The expression of a person sunk in thought is especially remarkable for the undetermined direction of the eyes'- the opposite of visual attention. Nor is there sufficient ground for the view that the intellectual picturing of an object in attention is always brought about through the revival of muscular experiences (Ribot, N. Lange, Philos. Studien, IV). Since muscular experiences involve space, and spacial experiences are most representable, it is true that many revivals are suggested by muscular revivals: but the evidence does not show that it is always so. Cf. James' interesting treatment of preperception-the apperceptive preparation of mind for the assimilation of a new object. Loc. cit., I. pp. $438 \mathrm{ff}$. 
to mental excitement. They are, moreover, common to reflex and voluutary attention and can be artificially produced. A brainless animal can be stimulated in such a way as to show the expression of high attention.

Effects Proper. The more legitimate effects of voluntary attention are the muscular contractions and inhibitions which follow it. Attempts are being made to bring these also under the conquest of artificial production: the belief being that volition as a self-determining thing will then go to the wall. Féré claims ' that the increase in force, rapidity, and precision of movements voluntarily attended to may be brought about by mechanical means (weights, high air-pressure, lying posture, etc.), the additional force coming from other parts of the system. Béclard contends that the extremest muscular tension is found in the immobility or static contraction characteristic of voluntary attention, and that the attention is this extreme expenditure of nervous force: he points to the fact that the diffusion and repose of attention is at once the relaxation of all muscular contraction down to the complete inactivity, on both sides, seen in sleep." Loeb' and others find that when definite motor centres are destroyed there is a prolonged period of inertia in the limbs affected; more voluntary effort has to be made to move them : this is held to indicate that effort is the drawing of nervous force from other regions.

As to the experimental endeavor, there is no reason that it should not be to some degree successful." Why shonld not there be-indeed must there not be?physical antecedent to every such physical change; and why may not physiology in some cases discover it? But when there is such an artificial production of the effects

1 Revue Philosophique, Oct. 1890.

Ibid., p. 401.

'Pflüger's Archiv, xxxix.

- Cf. above. Chap. I. $\S 3,3$. 
of attention, what does it prove concerning volition? It only proves that conservation holds in brain-activities, a position readily enough admitted. Volition might be the one law of mental development still, on either of the hypotheses already advanced to explain the relation of consciousness to the nervous system. ${ }^{2}$

Physical Control. The extremely complex system of checks and counter-checks which we call physical control, in adult life, has had a slow development. Assuming the directive influence of consciousness, becoming explicit in the early efforts of an infant, we find that it avails itself of the general sensori-motor law already noted under the head of suggestion. The basis of all consists in spontaneous, ${ }^{2}$ reflex, and instinctive ${ }^{4}$ movements. Such movements, when painful, tend to subside by the immediate inhibitive effect of pain. When pleasurable, by a parallel law, they tend to continue. Thus a link is formed between sensation and movement whereby memories of pleasures and pains become stimulants to adaptive reactions. Such a primitive law of self-preservation is seen in lower orders of life, where there is no deliberative choice, and where the conditions are such that a very narrow range of adaptations suffices to continue the creature's existence. But with the human infant this is altogether insufficient. ${ }^{5}$ The extraordinary complexity of the life for which he is destined renders necessary a mus-

${ }^{1}$ Above, Chap. XV. § 2.

Bain.

${ }^{3}$ Lotze, Spencer, Schneider : for a discussion of the rise of voluntary inhibition of reflexes, with experimental evidence, see Preyer, loc. cit., I. pp. 227-31.

'Sully : see his good discussion, Outlines, pp. $397 \mathrm{ff}$. Schneider's view really approximates Sully's, since the former derives reflexes from reactions originally accompanied by feeling.

- Against Bain, to whom the development so far is due. Senses and Intellect, pp. 301-11; Emotions and Will, pp. 303-379. 
cular pliability which cannot wait npon the exigencies of accidental or instinctive motor experience. Hence his long infancy is spent in strenuous effort. To his natural aversion to pain he adds deliberate contrivance to avert it; to suggestion he adds persistent imitation; to experience he adds voluntary experiment. And all his education is supported by instruction from without. The muscular system is thus brought under voluntary control generally, so far as to subserve the demands of life ; and in particular directions, farther, as employment or preference demands it.

Such control extends to the inhibition in part of many reflex functions, such as coughing, sneezing, shivering, etc., ${ }^{2}$ in some few instances to the automatic processes, and tends to break up instincts and dispose their elements differently. Only those muscles are available for will which have organic connection with the cerebrum. Some of the available muscles of the body, however, never come under voluntary control, because they are not of use. For example, the muscles of the ear may be made available for moving the ear voluntarily after repeated effort.

Moral Control.' Similarly the impulses and desires are brought under a law of reasomable activity. The lawless indulgences of childhood partly correct themselves by their natural penalties. But in this sphere conflicts between immediate and remote results render the pleasures and pains of experience altogether inadequate as a guide of life. The balancing of results which is the slow work of prudence is supplemented by the counsels and forced precepts of teacher and parent. Obedience is the schoolmaster to self-restraint. And gradually reverence for persons becomes reverence for

1 Orchansky, Arch. für Anat. u. Plıy8., 1889, p. 179.

- Sce Bain's excellent remarks, Emotions and Will, chap. Ix, and the remarks on impulse above, Chap. III. $\S 2$. 
moderation, and obedience passes into prudential control. Moral control is in its development closely connected with prudential ; but, as has been seen above, it finds its law of operation in the moral imperative which sets its own type of obedience and administers its own sanctions.

Further, just as physical control passes into the state of subconscious innervation and contraction necessary for the uprightness, due balancing, and habitual adjustments of the body, so with mental and moral control. The well-harmonized mental life is a life of regulated flow : imagination is adjusted to fact, association held in to the requirements of logical procedure, emotion restricted to its due impelling influence, will moderated by deliberation. All this is a gradual outcome, and the final result takes its coloring from the degree of mental equilibrium we consciously attain by our individual choices and efforts. Volitions conform more and more to the rule of a guiding intention, right or wrong. Just as in the sphere of sensuous feeling there is a fund of common fixed sensibility, cœnæsthesis; so in the mental sphere we find a similar fund of relatively permanent will-stimulus, a conceptual cœnæsthesis, so to speak, or temperament. Thus, also, moral choices become habitual, and rightness of choice passes into virtue of character.

It is in the disintegration of these voluntary motor intuitions and the loss of the inhibitions on which they depend, that disease of the will takes its rise. Its first manifestation is lack of control in one way or another. It may be due either to a diminution of the inhibitive process in some special brainarea, or to a decay of the co-ordinating and selecting function as a whole. Tendencies toward impulsive and capricious action, the reign of fixed ideas and monomanias, of suicide and murder, kleptomania, dipsomania, etc., all these are exaggerated instances of what we all feel in the "irritation" of extreme fatigue, and the sudden promptings of impulse which we are able to control but not to silence. ${ }^{\circ}$ The opposite

' See Ribot, Diseases of the Will, and James, loc. cit., Ir. pp. 537-546, for references. 
tendency is seen in the so-called "insanity of doubt;" a condition in which the inhibitive process is so developed that no mental decision is ever sufficient for coufident action. 'This again may take the form of general apathy and laissez-faire; or it may be confined to a particular reaction or choice, as in patients who are never sure on a point of question or matter of fact, but return to it again and again in abnormal repetition. ${ }^{2}$

\section{\& 6. Rational Aspects of Volition.}

Intuition of Power. The rise of the intuition of power has already been briefly indicated.' The above analy'sis of effort reveals to us the concrete fact-voluntary atten. tion-in which it ultimately rests. Whatever their metaphysical validity may or may not be, we reach the ideas of self-agency and other-agency through efforts of our own against resistances. Just as space and time are revealed as intuitions through intellectual synthesis, and just as ideals are felt apprehensions of truths which lie beyond intellectual construction, so in volition we must recognize a regulative principle of agency, or power, which is the essence of experiences characterized by the term "will."

Intuition of Obligation. The categorical nature of the feeling of obligation has also been noted above." We found that duty was imperative and, in its form of command, universal. Given the right, the must of our obligation to perform it is the most unequivocally binding thing that we mortals know. In other words, obligation is a regulative and constitutive principle of the activity of will.

On volition, eonsult : James, loc. cit., Il. chap. $\mathbf{x \times 1}$, and his references; Höffding, Outlines, viI, A and B; Volkmann, Lehrbuch,

1 An example is given by Knapp, Amer. Journ. Psych., Iu. p. 1.

Senses and Intellect, chap. Xv. $\$ 5$.

3 Chap. IX. \& 7. 
\$147-49; Rabier, Psychologie, chap. xxxvin; Sergi, Psych. Phys., liv. v. chaps. IV-V; Green, Prolegomena to Ethics, bk. II. chaps. I-II; Schneider, Mensch. Wille, chap. xIII; Sully, Outlines, chap. XIV; Spencer, Psychology, part IV, chap. IX, and Essays (1868), vol. I. pp. 300-324; Bain, Emotions and Will, part II. chaps. II-vII; Ward, loc. cit.; James Mill, Analysis, chaps. xxII-xxIv; Drbal, Lehrbuch, $\S \S 137-42$; Perez, First Three Years, chap. XII; Carpenter, Ment. Phys., b. I. chap. Ix, 2, 3, 4; Garnier, Traité des Facultés de l'Ame, bk. v; Féré, Physiologie de l'Attention, Revue Philos., 1890; Wundt, Philos. Studien, I, p. 337 ff.; (Moral Control) refs. by Dewey, Psychology, p. 416; Morell, Outlines, pt. vI; Fortlage, System der Psych., §§ 86-94; Edwards, Inquiry into the Will; Fouillée, La Liberté et le Déterminisme. See also the references on "voluntary movement" at the end of the preceding chapter.

Further Problems for Study :

Nature and limits of responsibility and freedom;

Physical process of volition;

Theories of volition;

Philosophical implications of the doctrine of volition. 


\section{APPENDIX A.}

\section{EDUCATIONAL PSYCHOLOGY : BIBLIOGRAPHY.}

THE following references on the applications of psychological truths, in the training of the youthful mind, may be of service to teachers. What teachers need is not a "system of pedagogy," but a living and sympathetic knowledge of children. The following titles are selected from a great many, both because they are accessible, and because the writer has found them suggestive.

On Educational Psychology: Sully, Outlines of Psychology (in locis) ; Ruldestock, Habit and its Importance in Education; Compayré, Lectures on Pedagogy ; Froebel, Education of Man; Guyau, Education and Heredity; Bain, Education as a Science; Perez, l'Education dès le Berceau; Egger, De l'Intelligence et du Langage chez les Enfants;' de Saussure, L'Education progressive; Lange, Ueber Apperception; Barth, Ueber den Umgang; Pfisterer, Pädagogische Psychologie; Wiese, Die Bildung des Willens; Rosenkranz, Philosophy of Education; McLellan, Applied Psychology; Marion, Psychologie appliquée à l'Education; Rosmini, Method in Education; Hopkins, Educational Psychology; Tate, Philosophy of Education (ed. Sheib) ; Warner, Lectures on Mental Faculty; Baldwin, Infant Psychology, Science, XVI (1890), p. 351 ff.; Yeomans, Culture of the Observing Powers of Children; Hall, Education of the Will, Princeton Review, 1882.

General Works on Pedagogy: Herbart, Pädagogik (Werke, ed. Hartenstein, vols. 10 and 11); Waitz, Allgemeine Pädagogik; ; Spencer, On Education; Fitch, Lectures on Teaching; Johonnot, Principles and Practice of Teaching; Thring, Theory and Practice of Teaching; Memeyer, Grundsälze

I I am informed that an English translation of this work is in preparation. 
der Erziehung; Strümpel, Psychologische Pädagogik, Schultze, Ucbersicht ïber Pädagogik; Schumann, Lehrbuch der Pädagogik (esp. vol. II) ; Browning (Oscar), Introduction to Educational Theories and Aspects of Education; Quick, Essays on Elucational Reformers; Sidgwick (A.), Lectures on Education; Fröhlich, Wissenschaftliche Pädagogik.

Bibliographies of Education: Diesterweg, Wegweiser zur Bildung (5th ed.); Hall and Mansfield, Bibliography of Education (to 1876 : has many minor errors); Bibliography in Pädlagog. Jahresbericht, 1885 ; Ollenden, Bibliographie de l'Enseignement primaire (esp. vol. III, Monographies pädagogiques, Paris, 1889) ; Sonnenschein's Cyclopedia of Education (ed. Fletcher, 1889).

\section{APPENDIX B.}

\section{DREAM-EXCITATION.}

THE direct influence of slight sense-stimuli upon the flow and make-up of our dream-consciousness is a well-known fact, which can be proved by artificial experiment (see Maury, Le Sommeil et les Rêves, p. $132 \mathrm{ff}$.), but which it is difficult to confirm under ordinary circumstances, since we seldom waken after a well-marked dream-experience in time to catch the stimulus, or without altering the stimulus by movement, etc. On the night of October $22 \mathrm{~d}$, I had a dream which perfectly fulfilled the conditions of this experiment. I fell asleep about eleven o'clock, and found myself with a companion in a wood, watching a number of wood-cutters at work. After looking at them tor some time, one of the workmen drew my attention quite suddenly by giving forth a strange sound, half-musical and half-speech, by which he seemed to be trying to express something to his neighbor; and the sound came with every blow of his axe in regular rhythm. The sound seemed to me distinctly familiar and yet very strange, and I turned to my friend and said, "What an apology for conversation !" Just as I spoke I awoke, and the sound of the peculiar tone of a clock down-stairs striking twelve broke in upon my consciousness. The four remaining strokes of the clock preserved exactly the rhythm of the wood-

${ }^{1}$ From Science, vol. xII (1888), No. 300. 
choppisr's axe; and not only so, but the sense of familiarity which had puzzled me in the dream was relieved with a glow of pleasure as I recognized the sound of the clock.

'This experience illustrates also the remarkable swiftness with which now sensations are assimilated to the character of a previous dream-consciousness. Before the clock began, the men were simply cutting, without order or distinction. But when the sound broke in it was at once accommodated to the scene by important modifications. One workman is singled out : he begins to ply lis axe in the regular time of the clockbeats, and to give forth a sound which preserves in its general character the peculiarities of the real sound. Now, since I experienced in the dream no less than four beats, us the rhythm was perfectly established and clear in my conscious. ness, and there remained four beats after I awoke, this whole accommodation must have taken place in the interval between the first and the fifth beat (for it was then twelve o'clock). I have since measured the interval between the strokes of the clock, and find it to be two seconds. 'The whole time from the first to the fifth bent was therefore eight seconds. From this should be taken the time occupied by the dazed state between dreaming and waking, - say, at least, an interval of from two to four seconds. There remains a period of four to six seconds as the time of accommodation. This may be called, in a very rough way, the reaction time for a complex case of constructive imagination ; for the constructive imagination is nothing more than the free play of images in forms of ideal composition, due to the influx of additions from the sensorium. There is no direct way of measuring this time in the waking state, since the attention interferes with the process.

\section{APPENDIX C.}

\section{NUMBER FORMS.}

A NUMBER of instances of Mr. Galton's "Number Forms" have been reported to the writer. The accompanying cut (see next page), kindly drawn out with great care by Mr. Courteney Thorpe, of Boston, the gifted actor, may serve to illustrate the peculiarity. The following description from Galton must suffice here. The reader will find the subject amply discussed in his book. 


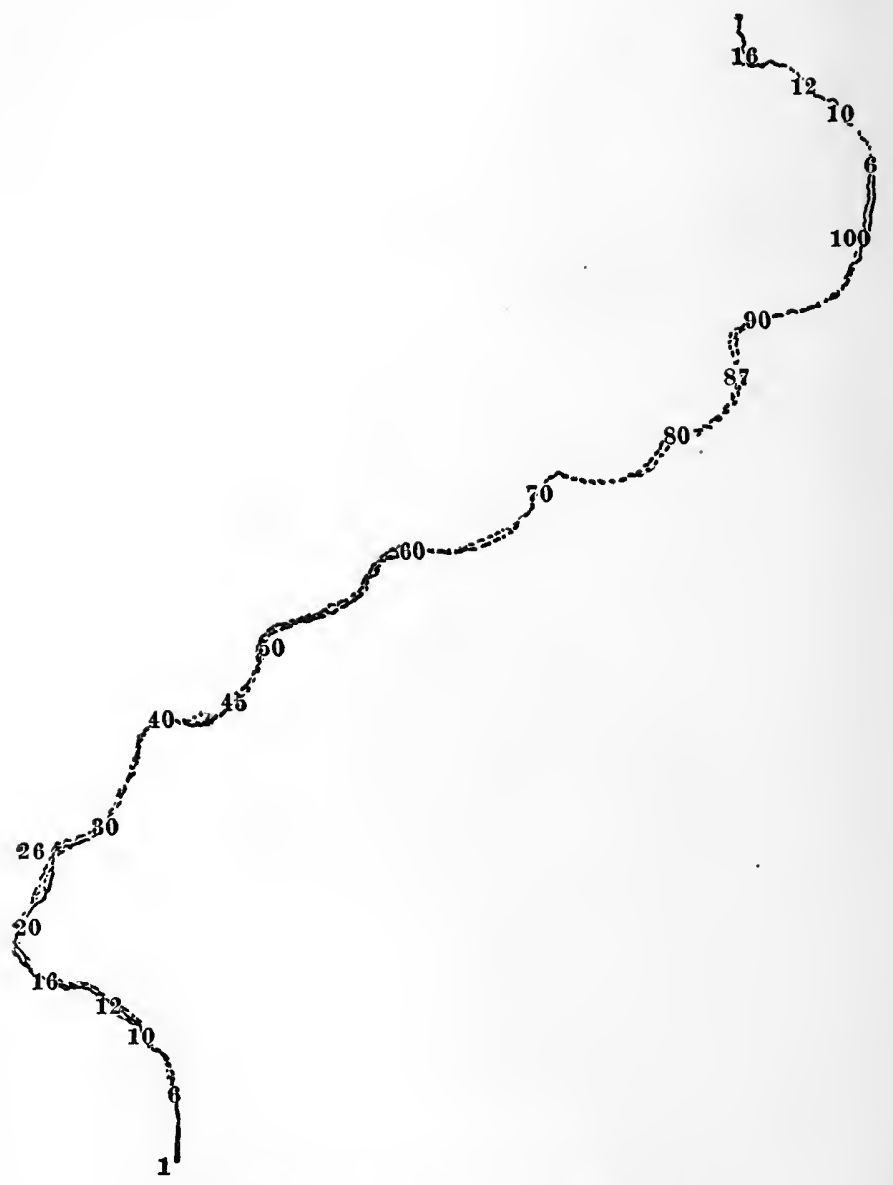

"Number form" of Mr. Courthney Thorpe. 
"The peculiarity in question is found, speaking very ronghly, in about 1 out of every 30 adult males and 15 females. It consists in the sudden and automatic appearance of a vivid and invariable 'form' in the mental field of view, whenever a numeral is thought of, and in which each numeral has its own definite place. This form may consist of a mere line of any shape, or a peculiarly arranged row or rows of figures, or of a shaded space." ' Among Americans, the "forms" seem to be much rarer than Galton's figures ( 1 in 30 and 1 in 15) would lead us to expect.

Inquiries into Human Facully. pp. 114 ff. 



\section{INDEX.}

Accommodation-law of nervous a., 49.

Activity-emotions of, $175 \mathrm{f}$.

Adjustment -as basis of tone, 124 ; emotions of, 176.

Esthetic-a. feeling, 233 fi.; a. judgment, 241.

Affect-the a., 313 ff., 354.

Allen (Grant)-on pleasure and pain, 128.

Alternatives -feeling of, 359.

Anasthesia (101).

Analgesia (101).

Analogies-of mental and nervous function, $66 \mathrm{ff}$.

Apperception-volitional a., 355.

Appetence (322 f.).

Aristotle-theory of pleasure and pain, 131.

Ascidian-n. system of, 16.

Association-a. tracts, 12-13; fig. ure of, 14; nervous vs. mental a., 68 \%.; a. In interest, 145; a. theory of reflex attention, 289.

Attention-feeling of reflex 8., 288 fif.

Automatic-a. reactions, 29; secondary a. reactions, 31 .

Aversion (330 1.).

BAIN - on unity of consciousness, 81; on pleasure and pain, 133 , 276; on belief, 172; on sympathy,
192; on fundamental emotions. 252; theory of volition, 344.

Bustian-on innervation-feelings, 349.

Beaunis - on motor consciousness, 39; on muscular feelings, 109; on innervation-feelings, 349 .

Béchard-on "static contraction," 338.

Belief (155 If.)-b. and doubt, 155 1.; b. in externai reality, 160 f.; in memory, $163 \mathrm{f}$; $\mathrm{b}$. in thought, 167; emotional b., 168; references on, 173 .

Bell-on emotional expression, 250.

Bianchi-on motor stimulation, 39.

Brentano-on belief, 158, 172.

Broadbent-on nervous action, 21.

Brown-Siquard-on function of hemispheres, 41.

Butler-on moral autbority, 212.

Canpenter - on second automatic reactions, 32.

Central-c. elements of nerrous system, 15 f.

Cerebellar-tracts, 9.

Character (363 ff.).

Choice (352 ff.).

Classification-o feelings, 88.

Column-sensory c., $8 \mathrm{fI}$; motor, $10 \mathrm{f}$.; lateral, 11. 
Conasthesis (99).

Conscience (228 f.).

Consciousness-nervous conditions of, $51 \mathrm{ff}$.; kinds of, $58 \mathrm{ff}$; passive c., $58 \mathrm{f}$.; reactive c., $60 \mathrm{f}$.; apperceptive and motor c., 63; voluntary, $63 \mathrm{ff}$; c. and retention, $66 \mathrm{f}$.; specific connection and c., $68 \mathrm{f}$.; summation and c., 69 ; c. and inhibition, $69 \mathrm{f}$.; unity of, $73 \mathrm{ff}$.; split-up c., 81;

"motor c., $280 \mathrm{ff}$.

Consent-as feeling, 64, 339.

Content-emotions of, $177 \mathrm{f}$.

Contrast-phenonena of, $91 \mathrm{f}$., 245.

Control-voluntary c., $378 \mathrm{ff}$.

Cortex-functions of, $40 \mathrm{ff}$.

Criterion-of mind, 52; of sentience, 56 ; of external reality, $160 \mathrm{ff}$.

DARwiN-on sympathy, 188; on emotional expression, 250.

Deliberation-feeling of, 64,357 .

Descartes-on pleasure and pain, 132.

Desire (320 ff.)-references on, 333.

Determinism - of will, $370 \mathrm{f}$.

Diffusion-nervous, 21; d. of pain, $116 \mathrm{f}$.; of emotion, 254.

Division-of ideal feelings, $173 \mathrm{ff}$.

Dömrich-on passive consciousness, 58.

Doubt-d. v8. belief, $155 \mathrm{f}$.

Dream-d. excitation, 384.

Dumont-on pleasure and pain, 129 ; on indifference, 278.

Duration-of emotion, $263 \mathrm{f}$.

Dynamogenesis - nervous, 28; mental, 281.

EducationaL-e. psychology, references, 383.

Effects-of volition, 376 ff.
Effort-feeling of, 64, 334 ff., 363. Electrical-e. feelings, 111.

Electricity-analogy with nerveaction, 19.

Emotion (174 ff.)-of activity, 174 f. ; of content, $177 \mathrm{f}$; ; of self, 179 f.; objective e., $180 \mathrm{ff}$; expressive e., 182 ff. ; sympathetic, 186 ; representative, 193; references on, 194, 243, 265; of relation, 195 ff. ; logical, 196 f.; conceptual, $198 \mathrm{ff}$; expression of, 249 . ff.; brain seat of, 255; duration of, $263 \mathrm{f}$.

End-organs, (7).

Engelmann-on protozoa, 18.

Ethical-e. feeling, 205 ff. ; e. judgment, 227.

Evolution-law of, 50.

Excitement-feeling as e., $244 \mathrm{f}$.

Expenditure-feeling of, 108, 286.

Exploration-interest as e., 141.

Expression-emotional, $249 \mathrm{ff}$.

Extensity-of feeling, 98.

External-e. reality-belief, $160 \mathrm{ff}$.

Fatigue (107 f.; 287).

Feeling-common f., 86, 89 ff.; definition of, 85; extensity of, 98 f.; common sensuous, $99 \mathrm{ff}$; organic, $100 \mathrm{f}$; ; cutaneous, 100 , 102; muscular, 100, 102 ff.; nervous, 100, 109 f.; kinæsthetic, $103 \mathrm{ff}$; innervation, $106 \mathrm{ff}$.; ideal f., $135 \mathrm{ff}$.; special ideal, $174 \mathrm{ff}$.; social, 193; f. for system, $204 \mathrm{f}$.; ethical, 205 ff. ; æsthetic, 233 ff.; references on, 194, 243; table of, 243; quantity of, $244 \mathrm{ff}$; of alternatives, 359 ; of freedom, $369 \mathrm{ff}$.; of responsibility, 375 .

Ferrier-on lateral columns, 11; on optical centre, 39 ; on pleasure and pain, 130.

Fiat-of will, $334 \mathrm{ff}$. 
Fortlage -on interest, 148.

Foster-on motor tracts, 8.

Frreodom-of the will, 869 fi.

Galton-on character and heredity, 367.

Ganglion-structure of. 16.

Georgo-on interest, 148.

Golgi-on nerve-cells, 6.

Goltz-exper. on braiuless animals, 25.

Groen (T. H.)-on passive consciousness, 58; on atoms of sensation, 96; on sympathy, 192.

HABIT-nervous, 24; law of, 49; interest arising from, 145; b. and movement, 331; h. and choice, 361.

Hamilion (Sir Wm.)-on pleasure and pain, $181 \mathrm{f}$.

Hedoniom (216 ff).

Helmholtz-on contrast, 82.

Herbart-division of emotions, 62.

Heredity (81 f.).

Herzen-on conditions of consclousness, $54 \mathrm{f}$.

His-on nerve-cells, 6.

Hodgon-on Ideal feeling, 186; on belief, 159, 172; on obligation; 222; on resthetic relations, 235.

Höfding-on sensations, 96; on sympathy, 191; on indifference, 278.

Horns-of spinal cord, 16.

Horvoicz-theory of attention, 841.

Eume-on knowledge, 48; on atoms of sensation, 95 ; on belief 164.

Hyperasthesia (101).

IDEALs-construction and nature off, $198 \mathrm{ff}$.

Imilation-suggestion by i., 299.
Impulse (804 II., 822 II., 815)

Indetorminism-of will, 869.

Inhorilance-Law of, 60.

Inhibition-nervous, 85 ff. ; mental, $69 \mathrm{II}$.

Initiation-of motives, $307 \mathrm{f}$.

Innorvation-feelings of, $106 \mathrm{fl}$., 348.

Instinct (308 fi.).

Integration-nervous, 28 1.; 1. and tone, 122.

Interest-as feeling, 189 ff.; Anal. ogy from, 49; 1 . and belief, 172; as will-stimulus, 316 f.

Intuition-feeling as 1., 202; 1 . of power and obligation, 881.

Intuitionaliom-of ethical end, 224.

Irritability-nervous, 18 ff.

JAMES (Wm.) - on criterion of mind, 52; on theory of sensation, 96 ; on reality-feeling, 150 ; on bellef, 173; on emotional expression, 256; on instinct, 811; on eflort, 842; on innervationfeeling8, 349.

Janet (Plerre)-on cmotional expression, 257.

Jessen-on fundamental emotions, 251: on passion, 257; on impulse, 307 ; on desire, 817 .

Judgment-moral, 227; asthetic, 242.

KANT-On atoms of sensation, 86; on pleasure and pain, 1331 .; on moral imperative, 210; on ethical end, 223.

Kinasthetic-k. feelings, 103 if.

Knonoledge-sensation and k. 93 ff. Kolliker-on sensory cells, 40; on nerve-ronuections, 6.

LANoB (C.)-on emotional expresslon, 256. 
Lawo-of nervous habit and accommodation, 49; of inheritance, 50 ; of evolution, 50.

Leibnitz-double-aspect theory, 54. Leroes-conception of nerve-action, $20 \mathrm{f}$.; on rise of consciousness, 51 ; on instinct, 311.

Lipps-on belief, 158.

Lotze-on mental unity, 80 ; on pleasure and pain, 133; on feeling as intention, 204; on indifference, 278; theory of consciousness, 345 .

Martinead-on springs of action, 333.

Maudsley-on unity of consciousness, $73 \mathrm{ff}$; on reduction of thought, 96.

Medulla_-oblongata, 11.

Meyer-M.'s experiment, 91.

Mill (Stuart)-on pleasure and pain, 132; on external reality, 166 ; on hedonism, 218 ; on obligation, 222.

Morgan (L.)-on conception, 196.

Mosso-on fatigue, 107.

Motwe (332 f.)-laws of, $352 \mathrm{ff}$.

Motor-unity of $\mathrm{m}$. consciousness,

74 ; m. consciousness, $280 \mathrm{ff}$. (references on) 293.

Movement-involuntary, $295 \mathrm{ff}$; voluntary, 316 ff., 334 ff.; refereuces on, 294, 350, 315.

Munk-on optical centre, 39.

Münsterberg-doctrine of sensation, 96,97 ; on innervation feelings, 349 .

Muscular-m. feelings, references on, $112 ; \mathrm{m}$. feelings as criterion of reality, 161.

NEgeT-of will, $334 \mathrm{f}$., $338 \mathrm{f}$.

Nervous-n. feelings, $109 \mathrm{f}$.

Neurility-as nervous property, $18 \mathrm{ff}$.
Neuroglia-function of, 5 .

Number-_" n.-forms," 385 f.

ObLigation-feeling of, 208 ; inteution of, 381.

Orris (S. S.)-on Plato's theory of pain, 130.

Orschansky-on physical base of will, $339,347$.

PaIN (and Pleasure)-113 ff. and $266 \mathrm{ff}$; theories of, $127 \mathrm{ff}$; references on, 134, 279 .

Passion (58 f.).

Patton (F. L.) -on "the good," 232.

Paulhan-on nature of emotion, 259.

Pflüger-experiments on brainless frogs, 25 ; “ avalanche " experiments, 20.

Pikler-ou external reality, 166.

Plato--on pleasure and pain, $130 \mathrm{f}$.

Pleasure (and pain)-113 ff. and $266 \mathrm{ff}$; theories of, $127 \mathrm{ff}$; references on, 134, 279; as stimuli to movement, $300 \mathrm{ff}$.

Potential-feeling of, 107, 287.

Power-intention of, 381.

Preyer-on origin of instinct, 311; on muscular movement, 349 .

Principle-ps. of nervous action, $38 \mathrm{ff}$; p. of specialization, $38 \mathrm{ff}$.; p. of indifference, 44; of substitution, $45 \mathrm{f}$.; of specific energies, 44; of substitution, $45 \mathrm{f}$.; nervous summation, $47 \mathrm{f}$; of specific connection, $47 \mathrm{f}$.

Protozoa-irritability of, 18.

Purpose (351).

Pyramids-of spinal cord, 10-12.

RABIER-on reality-feeling, 150; composite r., 169; self-ultimate r., 170. 
Roaction-nervous, kinds of, $28 \mathrm{ff}$.; automatic, 29; reflex, $30 \mathrm{ff}$.; vol. untary, 33 fl.; iuhibitive, 35 II.; secondary-automatic, 81 .

Reactive-r. consciousuess, 60 f., 285.

Reality-feeling of, 148 fi.; belicf in exterual r., $160 \mathrm{f}$.

References-on nervous system, $50-83$; on classif. of feclings, 88 ; on common feeling, 112; on muscular feeling, 112; on pleasure and pain, 194; on belicf, 173; on emotion, 194, 265; on conceptual feeling, 243; on feeling as indifference, 279; on motor consciousness, 294; on inpulse, instinct, suggestion, 815; on desire, 333 ; on voluntary movement, 350; ou volition, 881 .

Reflex-reactions, 30 ff.

Reid-on behalf, 171.

Relativity-of sense-qualities, 91 ; of pleasure and pain, 121, 126; of feeling, 244.

Representation-law of contradictory r., $70 \mathrm{f}$.

Resistanco-feelings of, as giving external reality, 162.

Responsibility-feeling of, 375.

Retention-nervous, 24 f.; r. and consciousness, $66 \mathrm{f}$.

Rhythm-of emotion, 265.

Ribot-doctrine of sensation, 96; theory of attention, 341; on function of consciousness, 348 .

Richol-on nervous conduction, 20.

Robertson (Croom) - on innervation-feelings, 848.

Romanes-on protozoa, 18; on reflex reactions of sea-urchins, 81; on criterion of mind, 52,345 ; on pleasure and pain. 128; on conception, 186; on origin of instinct, 810 .
Savant-8.'s wheel, 48.

Seluifor-on optleal ccutre, 80.

Schneider-on consciousuens, 55; on classification of feolings, 181 ; on emotion, 181; origin of iu. stinct, $\mathbf{8 1 0 .}$

Schrador-experiments on brinless pigeons, 25.

Self-emotious of, 179 f.

Senation-89 If.; s. and kuowledge, 83 tr.

Sensibility-8. and senticnce, 55 f.; nature of, 84 fi. ; divisions of, 88 ; references on, 88 .

Bonsory-8. nerve transmission, 7 fi.

Sensuous-common 8. feeling. 99 II.; divisions of sensuous feeling, 300; s. pleasure and pain, 113 If.

Sentience-as nervous property, 18, 22 II.; 8. and sensibility, 55 fi.; criterion of, $56 ; 8$. and the subconscious, 57.

Sidgwick-on moral authority, 212; on "intention," 228; on pleasure, 276.

Specialization-principle of nervous s., 88 f.

specific-energy of nerves, 44; principle of 8 . connection, $47 \mathrm{f}$.; 8. connection and consciousness, $68 \mathrm{f}$.

Spencer-on theory of sensation, 96; on space-perception, 59; on pleasure and pain, 128; on sympathy, 192; on obligation, 222; on origin of instinct, $\mathbf{3 1 0 .}$

Spinosa-on pleasure and pain, 182; on sympathy, 190; on will. 878.

Spiritual-s. theory of reflex attention, $292 \mathrm{f}$.

Sponlaneily-motor 8., 803.

Stimulus-summation of, 47 f.; 8. to Involuntary morement, 205 
ff.; to voluntary movement, 316 ff.

Stout-on belief in external reality, 166.

Structure-of nervous system, $1 \mathrm{ff}$. Subconscious - the s. and sentience, 57; motor value of the s., $283 \mathrm{f}$. Substitution-of nervous function, 45.

Suggestion-as motor stimulus, 297 ff.; references on, 315; as willstimulus, $318 \mathrm{f}$.

Sully-on "indifference," 276.

Summation-nervous summation, $47 \mathrm{f}$.; summation and consciousness, 69.

Sympathy-emotion of, $186 \mathrm{ff}$; morals, $20 \%$.

Theories-of pleasure and pain, $127 \mathrm{ff}$; ; $t$. of coefficient of belief, 167 ; of reflex attention, $289 \mathrm{ff}$.

ULRICI-on interest, 148.

Unity-organic theory of mental unity, $73 \mathrm{ff}$.
Unreality-feeling of, 151.

Urbantschitsch - on color-perception, 21.

Utilitarianism (219 ff.).

Volition (351 ff.)-apperception in $\mathbf{v}$., 355; references on, 381.

Volkmann (v. Volkmar)-on interest, 148; on desire, 328.

Voluntary- - . nervous reactions, $33 \mathrm{ff}$; v. consciousness, $63 \mathrm{ff}$.; v. movement, 334 .

WAITZ—on "indifference," 277. Ward-on extensity of feeling, 99; on pleasure and pain, 268 .

Watson (J.)-on sensation, 59.

Weber (E. H.)-principle of specific energies, 44.

Wundt-double-aspect of, 54; on æsthetic feeling, 234; on indifference, 278.

ZhIsING-Z.'s “ golden cut," 236. 


\section{THE AMERICAN SCIENCE SERIES.}

THE principal objects of the series are to supply the lack-in some subjects very great-of authoritative books whose principles are, so far as practicable, illustrated by familiar American facts, and also to supply the other lack that the advance of Sci. ence perennially creates, of text-books which at least do not contradict the latest generalizations. The scheme systematically outlines the field of Science, as the term is usually employed with reference to general education, and includes ADVANCED Courses for maturer college students, BRIEFER COURSES for beginners in school or college, and Elementary COURSEs for the youngest classes. The Briefer Courses are not mere abridgments of the larger works, but, with perhaps a single exception, are much less technical in style and more elementary in method. While somewhat narrower in range of topics, they give equal emphasis to controlling principles. The following books in this series are already published:

The human Body. By H. Newell Martin, Professor in the Johns Hopkins University.

Advanced Course. 8vo. 655 pp.

Designed to impart the kind and amount of knowledge every educated person should possess of the structure and activities and the conditions of healthy working of the human body. While intelligible to the general reader, it is accurate and sufficiently minute in details to meet the requirements of students who are not making human anatomy and physiology subjects of special advanced study. The regular editions of the book contain an appendix on Reproduction and Development. Copies without this will be sent when specially ordered.

From the Chicago Tribune: " The reader who follows him through to the end of the book will be tetter informed on the subject of moders physiology in its general features than most of the medical practitioners who rest on the knowledge gained in comparatively antiquated text.books, and will, if possessed of average good judgment and powers of discrimination, not be in any way confused by state ments of dubious questions or conflicting views." 


\section{THE HUMAN BODY,-Continued.}

\section{Briefer Course, I2mo. $364 \mathrm{pp}$.}

Aims to make the study of this branch of Natural Science a source of discipline to the observing and reasoning faculties, and not merely to present a set of facts, useful to know, which the pupil is to learn by heart, like the multiplication-table. With this in view, the author attempts to exhibit, so far as is practicable in an elementary treatise, the ascertained facts of Physiology as illustrations of, or deductions from, the two cardinal principles by which it, as a department of modern science, is controlled,-namely, the doctrine of the "Conservation of Energy" and that of the "Physiological Division of Labor." To the same end he also gives simple, practical directions to assist the teacher in demonstrating to the class the fundamental facts of the science. The book includes a chapter on the action upon the body of stimulants and narcotics.

From Henry Sewall, Professor of Physiology, University of Michi. gan: "The number of poor books meant to serve the purpose of text-books of physiology for schools is so great that it is well to define clearly the needs of such a work: $\mathbf{I}$. That it shall contain accurate statements of fact. 2. That its facts shall not be too numerous, but chosen so that the important truths are recognized in their true relations. 3. That the language shall be so lucid as to give no excuse for misunderstanding. 4. That the value of the study as a discipline to the reasoning faculties shall be continually kept in view. I know of no elementary text-book which is the superior, if the equal, of Prof. Martin's, as judged by these conditions."

\section{Elementary Course. I2mo. 261 pp.}

A very earnest attempt to present the subject so that children may easily understand it, and, whenever possible, to start with familiar facts and gradually to lead up to less obvious ones. The action on the body of stimulants and narcotics is fully treated:

From W. S. PERrY, Superintendent of Schools, Ann Arbor, Mich.: "I find in it the same accuracy of statement and scholarly strength: that characterize both the larger editions. The large relative space given to hygiene is fully in accord with the latest educational opinion and practice; while the amount of anatomy and physiology comprised in the compact treatment of these divisions is quite enough for the most practical knowledge of the subject. The handling of alcohol and narcotics is, in my opinion, especially good. The most admira. ble feature of the book is its fine adaptation to the capacity of younger pupils. The diction is simple and pure, the style clear and direct, and the manner of presentation bright and attractive." 
A8tronomy. By Simon Newcomb, Professor in the Johns Hopkins University, and EDWARD S. HOLDEN, Director of the Lick Observatory.

\section{Advanced Course. 8vo. 512 pp.}

To facilitate its use by students of different grades, the subject-matter is divided into two classes, distinguished by the size of the type. The portions in large type form a complete course for the use of those who desire only such a general knowledge of the subject as can be acquired without the application of advanced mathematics. The portions in small type comprise additions for the use of those students who either desire a more detailed and precise knowledge of the subject, or who intend to make astronomy a special study.

From C. A. Young, Professor in Princelon College: "I conclude that it is decidedly superior to anything else in the market on the same subject and designed for the same purpose."

\section{Briefer Course. $12 \mathrm{mo} .352 \mathrm{pp}$.}

Aims to furnish a tolerably complete outline of the astronomy of to-day, in as elementary a shape as will yield satisfactory returns for the learner's time and labor. It has been abridged from the larger work, not by compressing the same matter into less space, but by omitting the details of practical astronomy, thus giving to the descriptive portions a greater relative prominence.

From THR CRITIC: "The book is in refreshing contrast to the productions of the professional schoolbook-makers, who, having only a superficial knowledge of the matter in hand, gather their material, without sense or discrimination, from all sorts of authorities, and present as the result an indigesta moles, a mass of crudities, not unmixed with errors. The student of this book may feel secure as to the correctness of whatever he finds in it. Facts appear as facts. and theories and speculations stand for what they are, and are worth."

From W. B. Graves, Master Scientific Department of Phillips Academy: "I have used the Briefer Course of Astronomy during the past year. It is up to the times, the points are put in a way to interest the student, and the size of the book makes it easy to go over the subject in the time allotted by our schedule."

From Henry Lefavour. late Teacher of Astronemy, Williston Semi. nary: "The impression which I formed upon first examination, that it was in very many respects the best elementary text-book on the subject, has been confirmed by my experience with it in the clase room." 


\section{zOOLOaY. By A. S. PACKard, Professor in Brown Univer-} sity.

Advanced Course, 8vo. $719 \mathrm{pp}$.

Designed to be used either in the recitation-room or in the laboratory. It will serve as a guide to the student who, with a desire to get at first-hand a general knowledge of the structure of leading types of life, examines living animals, watches their movements and habits, and finally dissects them. He is presented first with the facts, and led to a thorough knowledge of a few typical forms, then taught to compare these with others, and finally led to the principles or inductions growing out of the facts.

From A. E. Verrill, Professor of Zoology in Yale College: "The general treatment of the subject is good, and the descriptions of structure and the definitions of groups are, for the most part, clear, concise, and not so much overburdened by technical terms as in several other manuals of structural zoology now in use."

Briefer Course, $12 \mathrm{mo} .334 \mathrm{pp}$.

The distinctive characteristic of this book is its use of the object method. The author would have the pupils first examine and roughly dissect a fish, in order to attain some notion of vertebrate structure as a basis of comparison. Beginning then with the lowest forms, he leads the pupil through the whole animal kingdom until man is reached. As each of its great divisions comes under observation, he gives detailed instructions for dissecting some one animal as a type of the class, and bases the study of other forms on the knowledge thus obtained.

From Herbert Osborn, Professor of Zoology, Iowa Agricultural College: "I can gladly recommend it to any one desiring a work of such character. While I strongly insist that students should study animals from the animals themselves, - a point strongly urged by Prof. Packard in his preface, - I also recognize the necessity of a reliable text-book as a guide. As such a guide, and covering the ground it does, I know of nothing better than Packard's."

First Lessons in Zoology. $12 \mathrm{mo} .290 \mathrm{pp}$.

In method this book differs considerably from those mentioned above. Since it is meant for young beginners, it describes but few types, mostly those of the higher orders, and discusses their relations to one another and to their surroundings. The aim, however, is the same with that of the others; namely, to make clear the general principles of the science, rather than to fill the pupil's mind with a mass of what may appear to nim unrelated facts. 
P8YChOLOOY-Advanced Courso. By William James, Professor in Harvard University. 2 vols. 8vo., 689, $704 \mathrm{pp}$.

From Prof. E. H. GRIFriN, John Hopkins University: "An Important contribution to psychological science, discussing its present aspects and problems with admirable breadth, insight, and independence."

From Prof. John Dewey, University of Michigan: "A remarkable union of wide learning, originality of treatment, and, above all, of never-failing suggestions. To me the best treatment of the whole matter of advanced psychology in existence. It does more to put psychology in scientific position both as to the statement of established results and a stimulating to further problems and their treatment, than any other book of which I know."

From Hon. W. T. HARRIS, National Bureau of Education: "I have never seen before a work that brings together so fully all of the labors, experimental and analytic, of the school of physiological psychologists."

Botany, By Charles E. Bessey, Professor in the University of Nebraska.

Advanced Course. 8vo. 6il pp.

Aims to lead the student to obtain at first-hand his knowledge of the anatomy and physiology of plants. Accordingly, the presentation of matter is such as to fit the book for constant use in the labaratory, the text supplying the outline sketch which the student is to fill in by the aid of scalpel and microscope.

From J. C. Arthur, Editor of The Botanical Gazetle: "The first botanical text-book issued in America which treats the most important departments of the science with anything like due consideration. This is especially true in reference to the physiology and histology of plants, and also to special morphology. Structural Botany and classification have up to the present time monopolized the feld, greatly retarding the diffusion of a more complete knowledge of the science."

Essentials of Botany. 12mo. $292 \mathrm{pp}$.

A guide to beginners. Its principles are, that the true aim of botanical study is not so much to seek the family and proper names of specimens as to ascertain the laws of plant structure and plant life; that this can be done only by examining and dissecting the plants themselves; and that it is best to confine the attention to a few leading types, and to take up first the simpler and more easily understood forms, and afterwards those whose structure and functions are more complex.

From J. T. Rothrock, Professor in the University of Pennsylvania: " There is nothing superficial in it, nothing needless introduced, nothing essential left out. The language is lucid; and, as the crowning merit of the book, the author has introduced throughout the volume 'Practical Studies,' which direct the student in his effort to for himself all that the text-book teaches." 
Chemistry. By Ira Remsen, Professor in the Johns Hopkins University.

Advanced Course. 8vo.

The general plan of this work will be the same with that of the Briefer Course, already published. But the part in which the members of the different families are treated will be considerably enlarged. Some attention will be given to the lines of investigation regarding chemical affinity, dissociation, speed of chemical action, mass action, chemical equilibrium, thermochemistry, etc. The periodic law, and the numerous relations which have been traced between the chemical and physical properties of the elements and their positions in the periodic system will be specially emphasized. Reference will also be made to the subject of the chemical constitution of compounds, and the methods used in determining constitution.

Introduction to the Study of Chemistry. 12mo. $389 \mathrm{pp.}$

The one comprehensive truth which the author aims to make clear to the student is the essential nature of chemical action. With this in view, he devotes the first 208 pages of the book to a carefully selected and arranged series of simple experiments, in which are gradually developed the main principles of the subject. His method is purely inductive; and, wherever experience has shown it to be practicable, the truths are drawn out by pointed questions, rather than fully stated. Next, when the student is in a position to appreciate it, comes a simple account of the theory of the science. The last $15^{\circ}$ pages of the book are given to a survey, fully illustrated by experiments, of the leading families of inorganic compounds.

From Arthur W. Wright, Professor in Yale College:-The student is not merely made acquainted with the phenomena of chemistry, but is constantly led to reason upon them, to draw conclusions from them, and to study their significance with reference to the processes of chemical action-a course which makes the book in a high degree disciplinary as well as instructive.

From Thos. C. VAN Nuys, Professor of Chemistry in the Indiana University:-It seems to me that Remsen's "Introduction to the Study of Chemistry" meets every requirement as a text or class book.

From C. Les Mees, Professor of Chemistry in the Ohio University: -I unhesitatingly recommend it as the best work as yet published for the use of beginners in the study. Having used it, I feel justified in saying this much. 


\section{CHEMISTRY-Continued.}

Elements of Chemlstry, 12mo. $272 \mathrm{pp}$.

Utilizes the facts of every-day experience to show what chemistry is and how things are studied chemically. The language is untechnical, and the subject is fully illustrated by simple experiments, in which the pupil is led by questions to make his own inferences. The author has written under the belief that "a rational course in chemistry, whether for younger or older pupils, is something more than a lot of statements of facts of more or less importance; a lot of experiments of more or less beauty : or a lot of rules devised for the purpose of enabling the pupil to tell what things are made of. If the course does not to some extent help the pupil to think as well as to see it does not deserve to be called rational."

Chasz Palmer, Professor in the Stale Normal Sthool, Salem, Mass.: -It is the best introduction to chemistry that I know, and I intend to put it into the hands of my pupils next Fall.

A. D. Gray, Instructor in Spring field (Mass.) High School - Neat, attractive, clear, and accurate, it leaves little to be desired or soughe for by one who would find the best book for an elementary course in our High Schools and Academies.

aeneral biolocy. By William T. Sedgwick, Professor in the Mass. Institute of Technology, and EDMUND B. WILSON, Professor in Bryn Mawr College. Part 1. 8vo. 193 pp.

This work is intended for college and university students as an introduction to the theoretical and practical study of biology. It is not zoology, botany, or physiology, and is intended not as a substitute, but as a foundation, for these more special studies. In accordance with the present obvious tendency of the best elementary biological teaching, it discusses broadly some of the leading principles of the science on the substantial basis of a thorough examination of a limited number of typical forms, including both plants and animals. Part First, now published, is a general introduction to the subject illustrated by the study of a few types. Part Second will contain a detailed survey of various plants and animals.

W. G. Farlow, Professor in Harvand University, Cambridge, Mass.: - An introduction is always difficult to write, and I know no work in which the general relations of plants and animals and the cell-structure have been so well stated in a condensed form. 
POLITICAL ECONOMY. By Francis A. Walker, President of the Massachusetts Institute of Technology.

Advanced Course. 8vo. 537 pp.

The peculiar merit of this book is its reality. The reader is brought to see the application of the laws of political economy to real facts. He learns the extent to which those laws hold good, and the manner in which they are applied. The subject is divided, as usual, into the three great branches of production, exchange, and distribution. An interesting and suggestive "book" on consumption is added, which serves to bring in conveniently the principles of population. The last part of the volume is given to the consideration of various practical applications of economic principles.

From Richmond Mayo Smith, Professor in Columbia College, $N . Y .:-$ In my opinion it is the best text-book of political economy that we as yet possess.

From Woodrow Wilson, Professor in Princeton University, N. J.: - It serves better than any other book I know of as an introduction to the most modern point of view as to economical questions.

Briefer Course. $12 \mathrm{mo} .415 \mathrm{pp}$.

The demand for a briefer manual by the same author for the use of schools in which only a short time can be given to the subject has led to the publication of the present volume. The work of abridgment has been effected mainly through excision, although some structural changes have been made, notably in the parts relating to distribution and consumption.

From Alexander Johnston, late Professor in Princeton University, N. J.:-Using the "Briefer Course" as a text-book, suited to any capacity, I am able at the same time to recommend the "Advanced Course" to those who are better able to use it as a book of reference, or more inclined to carry their work further.

Elementary Course. 12mo. $323 \mathrm{pp}$.

What has been attempted is a clear arrangement of topics; a simple, direct, and forcible presentation of the questions raised; the avoidance, as far as possible, of certain metaphysical distinctions which the author has found perplexing; a frequent repetition of cardinal doctrines, and especially a liberal use of concrete illustrations, drawn from facts of common experience or observation.

\section{HENRY HOLT \& CO., PUBLISHERS, N. Y.}


•

.

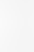




\section{University of Toronto}

Library

\section{DO NOT}

\section{REMOVE}

THE

CARD

FROM

THIS

\section{POCKET}

Acme Litinary Card Pocket Under Pat "Rel. Inder File" Made by LIBRARY BUREAU 
15itum

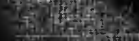

Dis

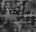

The

की 